\title{
Wetting and coalescence: beyond single-phase flows
}

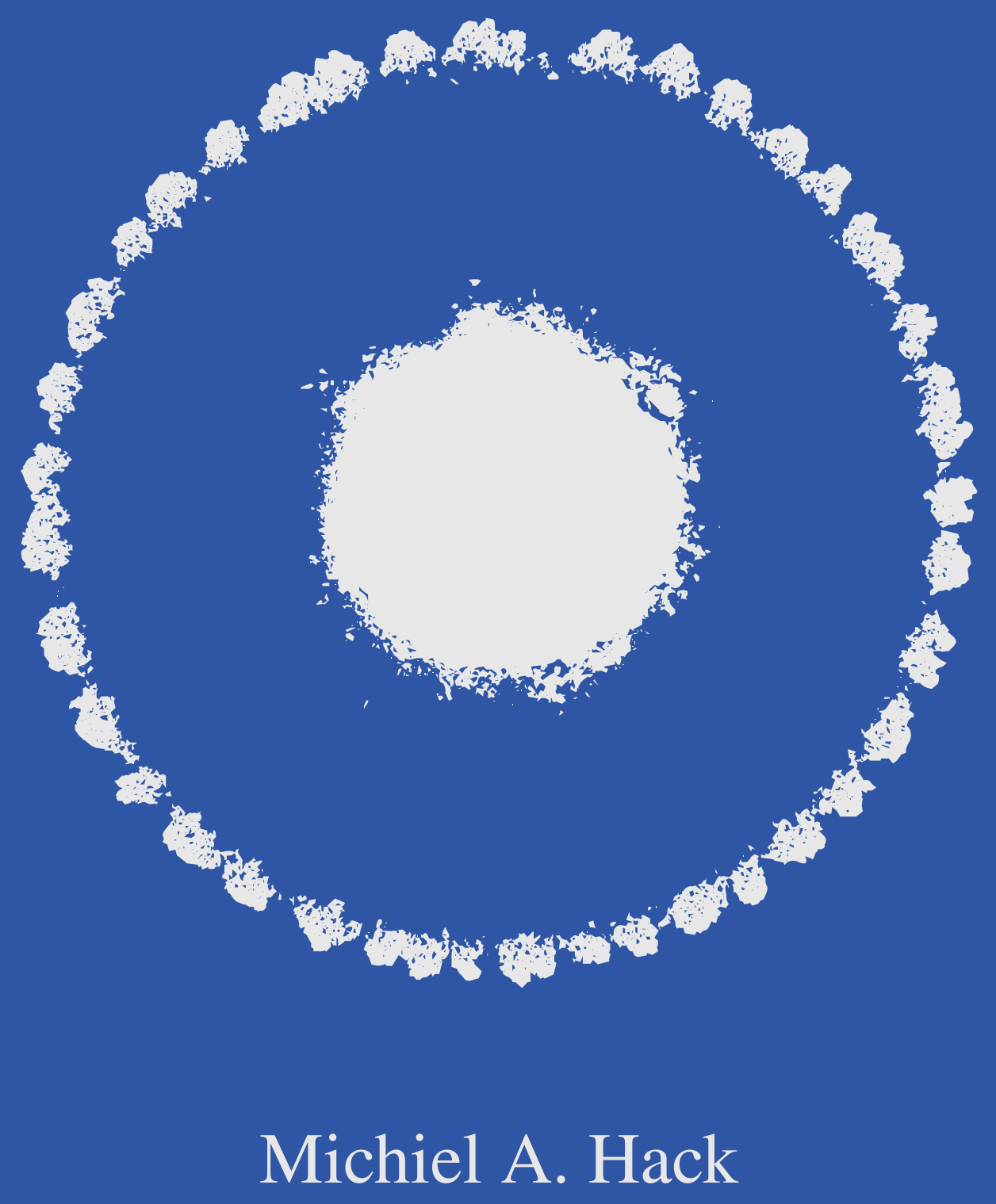





\section{Wetting and coalescence: \\ beyond single-phase flows}

Michiel Alexander Hack 
Thesis committee members:

prof. dr. J. L. Herek (chairman)

University of Twente

prof. dr. J. H. Snoeijer (promotor)

University of Twente

asst. prof. dr. T. J. Segers (copromotor)

prof. dr. K. Dalnoki-Veress

prof. dr. J. van der Gucht

dr. M. N. van der Linden

prof. dr. C. H. Venner

prof. dr. D. Lohse

University of Twente

McMaster University

Wageningen University \& Research Canon Production Printing B.V.

University of Twente

University of Twente

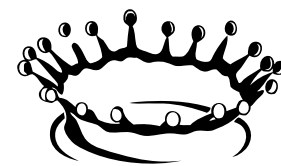

Physics of Fluids

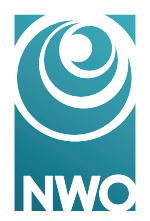

\section{UNIVERSITY OF TWENTE.}

The work in this thesis was carried out at the Physics of Fluids group of the Faculty of Science and Technology of the University of Twente. It is part of an Industrial Partnership Programme of the Netherlands Organisation for Scientific Research (NWO), cofinanced by Canon Production Printing Netherlands B.V., University of Twente, and Eindhoven University of Technology

Dutch title:

Bevochtiging en samenvloeiing: voorbij enkelfasige stromen

Publisher:

Michiel Alexander Hack, Physics of Fluids, University of Twente, P.O. Box 217, 7500 AE Enschede, The Netherlands

Copyright @ 2021 Michiel Alexander Hack. All rights reserved.

No part of this work may be reproduced or transmitted for commercial purposes, in any form or by any means, electronic or mechanical, including photocopying and recording, or by any information storage or retrieval system, except as expressly permitted by the publisher.

ISBN: 978-90-365-5242-4

DOI: $10.3990 / 1.9789036552424$ 


\title{
WETTING AND COALESCENCE: BEYOND SINGLE-PHASE FLOWS
}

\author{
PROEFSCHRIFT
}

ter verkrijging van

de graad van doctor aan de Universiteit Twente, op gezag van de rector magnificus, prof. dr. ir. A. Veldkamp, volgens besluit van het College voor Promoties, in het openbaar te verdedigen op vrijdag 29 oktober 2021 om 14:45 uur

door

Michiel Alexander Hack geboren op 9 juli 1994 te Heemskerk, Nederland 
Dit proefschrift is goedgekeurd door de promotor:

\author{
prof. dr. J. H. Snoeijer
}

en door de copromotor:

asst. prof. dr. T. J. Segers 
To my parents. 



\section{Contents}

1 Introduction 1

1.1 Wetting: from Young's law to multi-phase flows . . . . . . . . 3

1.2 Drop break-up and coalescence: scaling laws and self-similarity 6

1.3 Fluid dynamics inspired by inkjet printing: a guide through the thesis ........................ 11

$\begin{array}{lll}\text { I Wetting } & 15\end{array}$

2 Printing wet-on-wet: Attraction and repulsion of drops on a $\begin{array}{ll}\text { viscous film } & 17\end{array}$

2.1 Introduction . . . . . . . . . . . . . . . . . . 18

2.2 Drop-induced surface deformation . . . . . . . . . . . . 19

2.3 Interaction between two drops . . . . . . . . . . . . . . 21

2.4 Conclusion and outlook . . . . . . . . . . . . . 26

3 Ring-shaped colloidal patterns on thin saline water films 29

3.1 Introduction . . . . . . . . . . . . . . . 30

3.2 Experimental methods . . . . . . . . . . . . . . . . 32

3.3 Ring-shaped patterns . . . . . . . . . . . . . . . . 35

3.4 Dynamical aspects of pattern formation . . . . . . . . . 40

3.5 Cluster shape and proposed mechanism . . . . . . . . . . 45

3.6 Conclusion and outlook . . . . . . . . . . . . . . 48

3.7 Appendix . . . . . . . . . . . . . 50

4 Wetting of two-component drops: Marangoni contraction versus autophobing $\quad 53$

4.1 Introduction . . . . . . . . . . . . . . . 54

4.2 Experimental methods . . . . . . . . . . . . 56 
4.3 Marangoni contraction . . . . . . . . . . . . . . . . 59

4.4 Autophobing . . . . . . . . . . . . . . 6 60

4.5 Effect of the molecular structure . . . . . . . . . . 63

4.6 Conclusion and outlook . . . . . . . . . . . . . 65

4.7 Appendix ....................... 67

$\begin{array}{ll}\text { II Coalescence } & 71\end{array}$

5 Self-similar liquid lens coalescence $\quad 73$

5.1 Introduction . . . . . . . . . . . . . . . . . 74

5.2 Experimental methods . . . . . . . . . . . . . . . . . . . 74

5.3 Coalescence dynamics . . . . . . . . . . . . 76

5.4 Viscous and inertial similarity solutions . . . . . . . . . . 77

5.5 Crossover . . . . . . . . . . . . . . . . 81

5.6 Conclusion and outlook . . . . . . . . . . . . 82

5.7 Appendix ........................ 83

6 Theory for the coalescence of viscous lenses $\quad 87$

6.1 Introduction . . . . . . . . . . . . . . . . 88

6.2 The viscous thin-sheet equations . . . . . . . . . . . . . . . . 91

6.3 The inner region . . . . . . . . . . . . . . . . . 94

6.4 The outer region and matching . . . . . . . . . . . 98

6.5 Conclusion . . . . . . . . . . . . . . . . . . . 101

6.6 Appendix . . . . . . . . . . . . . . . . . . . . 104

$\begin{array}{lll}7 & \text { When elasticity affects drop coalescence } & 107\end{array}$

7.1 Introduction . . . . . . . . . . . . . . . . . 108

7.2 Experimental methods . . . . . . . . . . . . . . . . . . 109

7.3 Time . . . . . . . . . . . . . . . . . 111

7.4 Space . . . . . . . . . . . . . . . . . . 112

7.5 Viscoelastic singularity . . . . . . . . . . . . . . . . . . 114

7.6 Viscoelastic self-similarity . . . . . . . . . . . . . . 116

7.7 Conclusion and outlook . . . . . . . . . . . . . . 116

7.8 Appendix . . . . . . . . . . . . . . . . 118

8 The asymmetric coalescence of two droplets with different surface tensions is caused by capillary waves 125

8.1 Introduction . . . . . . . . . . . . . . . . 126 
Contents

8.2 Methods . . . . . . . . . . . . . . . . . . . 127

8.3 Collisions between droplets of different surface tensions . . . . . 133

8.4 Asymmetric capillary waves . . . . . . . . . . . . . . 135

8.5 Conclusion and outlook . . . . . . . . . . . . . . . . 144

8.6 Appendix . . . . . . . . . . . . . . 146

9 Conclusion and outlook 149

9.1 Wetting . . . . . . . . . . . . . . . 150

9.2 Coalescence . . . . . . . . . . . . . . . . . 152

$\begin{array}{ll}\text { Bibliography } & 155\end{array}$

$\begin{array}{ll}\text { Summary } & 177\end{array}$

$\begin{array}{ll}\text { Samenvatting } & 181\end{array}$

$\begin{array}{ll}\text { Acknowledgements } & 185\end{array}$ 



\section{Chapter 1}

\section{Introduction}

The study of fluid mechanics covers a vast range of length-scales, spanning from the smallest blood vessels found in the human body to large atmospheric flows on Earth $[1,2]$. In this thesis, we focus on interfacial flow phenomena with length-scales that are smaller than approximately one millimetre. Such flows are typically driven by surface tension - a property of liquids that manifests itself at interfaces. A liquid molecule at an interface experiences roughly half the attractive interactions with other liquid molecules compared to a molecule in the bulk of the liquid. Nature therefore dictates that the surface area of a liquid interface should always be minimised, such that the energetic cost associated with the interface is minimal. Ample examples of the action
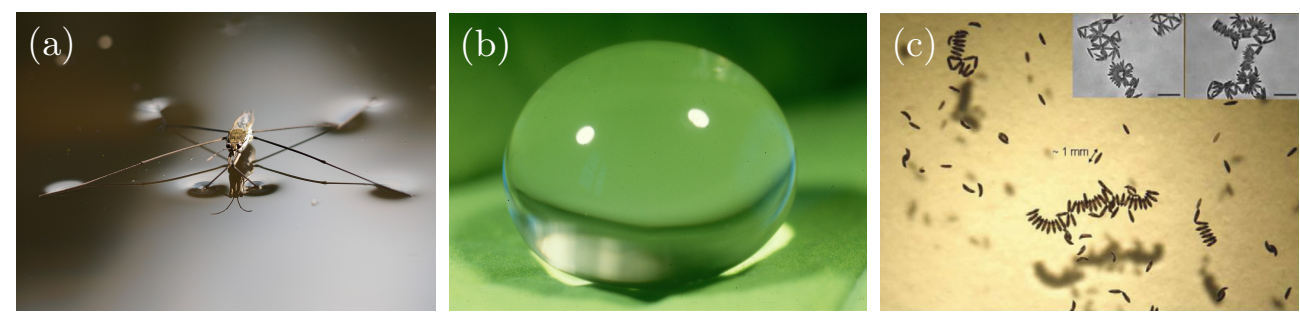

Figure 1.1: (a) A water strider at rest on the surface of a pond, its weight is supported by surface tension. Image taken from [3]. (b) A raindrop on the leaf of a lotus plant . The surface of the leaf is hydrophobic (water-repellent), resulting in a large contact angle. Image taken from [4]. (c) Floating mosquito eggs aggregate by capillary interactions to form egg clusters. Image taken from [5]. 
of surface tension can be found among the flora and fauna of nature: water striders can walk on water due to surface tension (Fig. 1.1a), raindrops take on a spherical shape when at rest on lotus plant leaves due to the minimisation of surface energy (Fig. 1.1b), and mosquito eggs aggregate by capillary interactions (Fig. 1.1c) [5].

Surface tension-driven flows also play an important role in many industrial applications. For example, the optical resolution of lithography in chip manufacturing can be increased by putting water between the lens and the silicon wafer, introducing a three-phase contact line [6]. Another example, and the main source of inspiration for the work presented in this thesis, is inkjet printing: a technology that allows controlled deposition of ink drops on a substrate with applications reaching far beyond document printing [7-10]. Many processes involved in inkjet printing are predominantly driven by surface tension. For example, an ink jet breaks up into drops by the Rayleigh-Plateau instability to minimise its surface energy (Fig. 1.2), and ink is absorbed by the porous structure of paper due to capillary action $[11,12]$. These flows, by their nature, involve multiple phases (ink is composed of multiple complex components), and are typically out of equilibrium $[13,14]$. A fundamental understanding of surface-tension driven multi-phase flows is therefore expected to aid in the further development of inkjet printing technology.

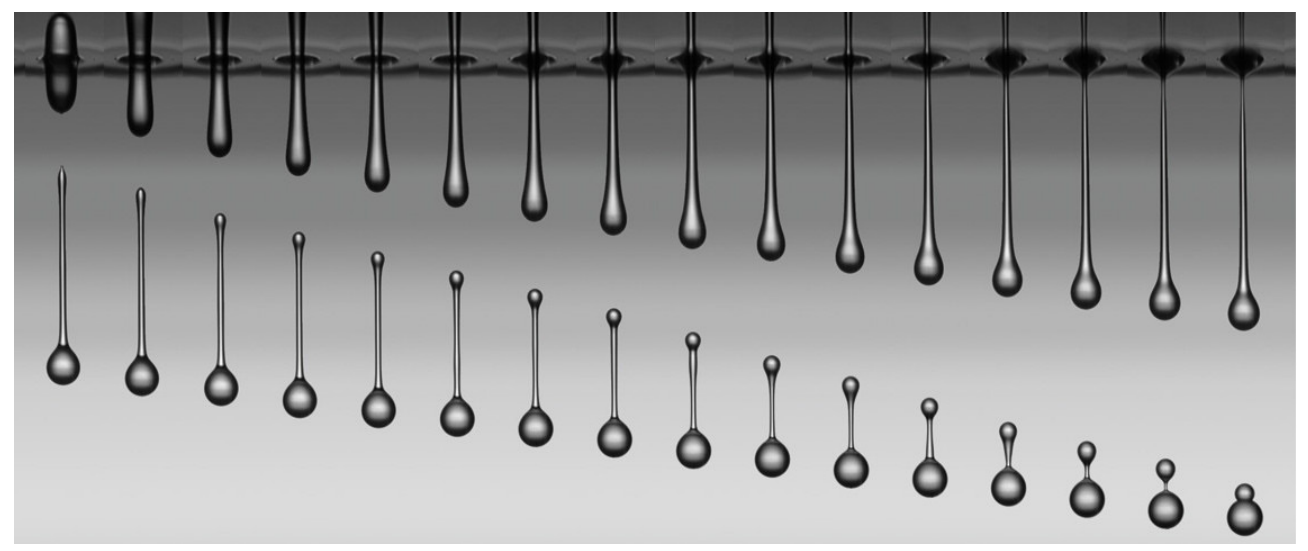

Figure 1.2: Time-sequence of picolitre-sized drops (diameter $\approx 15 \mu \mathrm{m}$ ) jetted from an inkjet printing nozzle. The tail of the drop retracts during flight to form a spherical drop, an example of the minimisation of surface energy. Image taken from [15]. 


\subsection{Wetting: from Young's law to multi-phase flows}

One of the key milestones in the study of surface tension was achieved by Thomas Young in 1805, when he proposed that the angle between the liquidsolid and the liquid-vapour interfaces of a drop resting on a solid substrate is given by

$$
\cos \theta=\frac{\gamma_{\mathrm{SV}}-\gamma_{\mathrm{SL}}}{\gamma_{\mathrm{LV}}} .
$$

In this expression $\gamma$ is the surface tension, and the subscripts denote the solidvapour, solid-liquid, and liquid-vapour interfaces, respectively, see Fig. 1.3 [16]. An example of a drop with a large contact angle is the raindrop shown in Fig. 1.1b, a striking feature of which is its spherical shape. Nearly all drops take the shape of a spherical cap when at equilibrium, due to nature's ability to minimise surface energy - the shape with the smallest surface area for any given volume is a sphere. There is, however, an important assumption underlying this spherical shape: drops must be small enough for gravity not to play a role. Gravity can be neglected when the length scale of the drop (typically the drop radius) is smaller than the capillary length $l_{c}=(\gamma / \rho g)^{1 / 2}$, where $\rho$ is the density of the liquid and $g$ is the gravitational acceleration. The capillary length, more generally, puts an upper limit on the type of flow problems that can be considered "small scale", since $l_{c}$ is typically on the order of a few millimetres (for example, $l_{c} \approx 2.7 \mathrm{~mm}$ for water). In this thesis, we restrict ourselves to small-scale flow phenomena with length scales smaller than $l_{c}$.

While Young's equation provides an elegant description of the contact line of a static drop consisting of a simple liquid such as water resting on a perfectly smooth substrate, it fails to predict the contact angle in many complex cases,

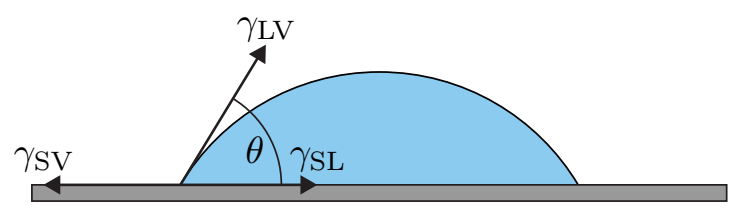

Figure 1.3: Definition of the contact angle $(\theta)$ of a sessile drop on an undeformable solid substrate. The contact angle is given by Young's equation, which describes the balance between the liquid-vapour $\left(\gamma_{\mathrm{LV}}\right)$, solid-liquid $\left(\gamma_{\mathrm{SL}}\right)$, and solid-vapour $\left(\gamma_{\mathrm{SV}}\right)$ surface tensions at equilibrium. 
which often involve multi-component liquids or non-equilibrium states. For example, the contact angles of drops in motion, drops on deformable substrates, and multi-component drops are in general not described by Eq. 1.1, since flow may arise near the contact line [17-19]. These cases, however, have an enormous significance, since numerous liquids used in industrial applications and everyday life, such as paints, pesticides, soaps, and various food products, consist of multiple components. Likewise, the inks used in inkjet printing are complex liquids containing various components such as water, pigments, viscosity modifiers, surfactants, humectants, buffers, and biocides [13]. Each of these components can affect the flow of the ink, be it during the jetting from a nozzle, during the merging of two neighbouring ink drops, or during any other stage of the printing process $[7,8,20]$. Separate components may also interact with each other, resulting in entirely new flow phenomena [14]. Moreover, ink drops are typically out of equilibrium, for example due to their motion or due to their evaporation. A proper understanding of the joint effects of each component on the wetting properties and flow dynamics of drops is therefore crucial for the effective application of inkjet printing.

A key phenomenon in the study of multi-phase interfacial flows is the so-called "Marangoni effect", which arises when two liquids of different surface tensions come into contact. The imbalanced surface tension introduces a tangential stress on the surface, which drives an interfacial flow. In turn, the
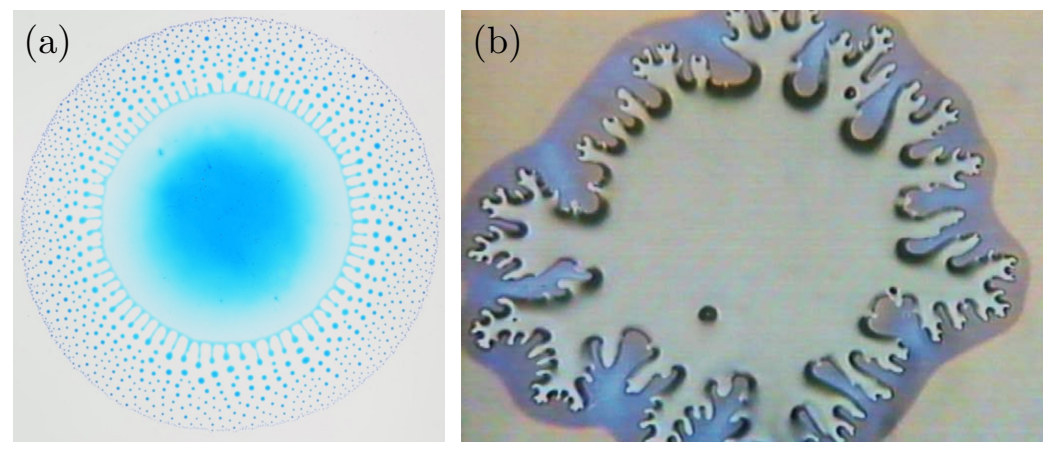

Figure 1.4: (a) A dyed drop consisting of a water-alcohol mixture deposited on a sunflower oil bath spontaneously fragments into smaller drops due to Marangoni flow. Image taken from [21]. (b) A fingering instablity is observed for a surfactant-containing drop deposited on a solvent-coated substrate. Image taken from [22]. 


\subsection{Wetting: from Young's law to multi-phase flows}

interfacial flow often induces a bulk flow, such that flow dynamics are completely different when the Marangoni effect is present compared to when it is not. Marangoni flow can also appear in initially well-mixed multi-component drops due to selective evaporation [19]. This can lead to intricate phenomena, such as phase segregation and pattern formation [23,24]. Another example of evaporation-driven Marangoni flow is "Marangoni bursting", see Fig. 1.4a [21,25]. Here, a drop consisting of a water-alcohol mixture is deposited on an oil bath. The contact angle is initially equal to zero, such that the drop takes a flat pancake-like shape. Evaporation strongly affects the local composition of the drop due to its small thickness, with more alcohol (low $\gamma$ ) evaporating at the contact line than water (high $\gamma$ ). The resulting imbalance of surface tension drives a Marangoni flow across the interface towards the edge of the drop, which breaks up into many small drops by the Rayleigh-Plateau instability. The expelled drops, consisting mainly of water, form contact angles larger than zero on oil, leading to intricate fragmentation patterns that underline the importance of surface tension in multi-phase flows.

A commonly used method to induce Marangoni flow is the addition of surfactant molecules ("surface-active agents") to a base liquid. These molecules tend to adsorb at interfaces due to their amphiphilic nature (the molecule is partly hydrophilic, and partly hydrophobic), thereby decreasing the surface tension of the liquid. Complex flow phenomena can be observed in surfactant-containing liquids, often driven by the Marangoni effect. For example, a surfactant drop deposited on a solvent-coated substrate forms meandering structures called fingers, due to a surface-tension driven instability, see Fig. 1.4b [22]. In inkjet printing, surfactants are often used to achieve good jetting and spreading properties of the ink, though they can also disturb the jetting process by causing unwanted Marangoni flow on the nozzle [13,26].

Finally, the physico-chemical interactions between separate components must be taken into account for an accurate description of multi-phase drop dynamics [14]. For example, the merging of two liquid chemical reactants can lead to intricate precipitation patterns, which also affect the flow dynamics, see Fig. 1.5a [27]. Similarly, ink is subject to physico-chemical processes during the printing process. The colour of ink is provided by pigments - insoluble colloidal particles. These particles, typically of the order of $100 \mathrm{~nm}$ to several micrometres in size, are small enough to be transported by the flow in drops. In evaporating drops, for example, particles aggregate at the contact line to form the well-known "coffee-ring effect", as shown in Fig. 1.5b [28,29]. In turn, friction between the particles can also affect the flow inside the drop [30]. De- 


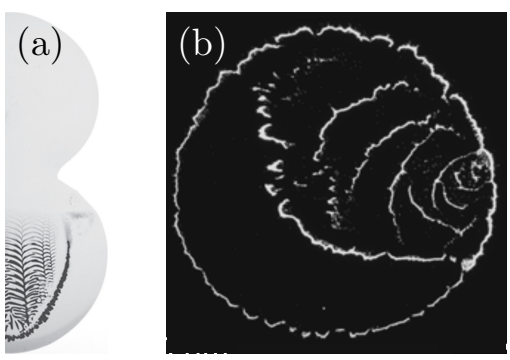

Figure 1.5: (a) Coalescence between two chemical reactants can lead to intricate precipitation patterns. Image taken from [27]. (b) Evaporating colloidal drops leave ring-shaped particle deposits. This is known as the "coffeering effect". Image taken from [29].

position patterns such as the coffee-ring effect are a key area of research in the context of inkjet printing, where small concentrated particle deposits are desired and spread-out ring-shaped deposition patterns are unwanted [31-33]. Additionally, pigment particles may follow the flow of the liquid into the porous structure of paper, resulting in pale colours [34]. Fast aggregation of pigment particles is therefore expected to improve print quality, since large particle clusters are less capable of following the flow of the liquid. Particle aggregation rates can be controlled by physico-chemical mechanisms - they can be increased by introducing electrolytes into the ink [34]. This is achieved by printing the ink onto a salt-containing "primer layer", such that pigment particles cluster immediately upon deposition [13]. Unable to follow the flow into the porous structure of the paper, the clustered pigment remains on the paper surface, resulting in vibrant colours. Hence, physico-chemical hydrodynamics are at the heart of inkjet printing.

\subsection{Drop break-up and coalescence: scaling laws and self-similarity}

Drops can undergo two elementary topological transitions. They can "break up", i.e., split up into two or more smaller drops (Fig. 1.6a). And, inversely, two drops can "coalesce" to form a single larger drop (Fig. 1.6b). These transitions are of fundamental importance in inkjet printing - ink jets break up into ink drops (Fig. 1.2) that eventually land on the substrate, where 
(a)

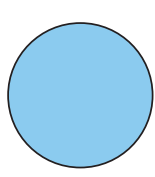

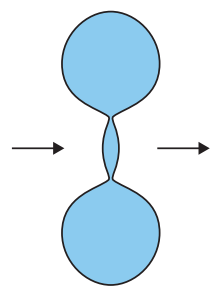

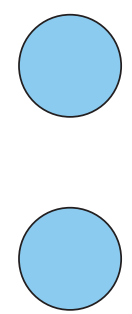

(b)

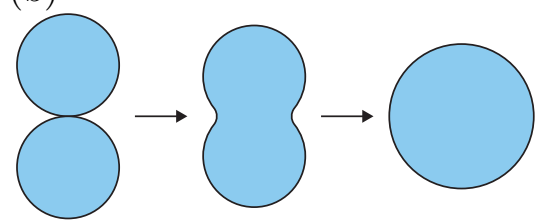

Figure 1.6: The two elementary topological transitions that drops can undergo. (a) Break-up of a single drop into two separate smaller drops. (b) The coalescence of two separate drops forms a single larger drop.

they coalesce with previously printed ink drops. A solid understanding of drop break-up and coalescence is therefore of paramount importance for inkjet printing technology.

Break-up (Fig. 1.6a) has been the subject of intense investigation over the past decades $[35,36]$. When a drop splits into smaller drops, a liquid thread that gradually thins over time is formed. Eventually this leads to a singularity - when the thread approaches zero thickness, the local curvature and Laplace pressure blow up [37]. When the thread reaches zero thickness the drops separate completely. This is called pinch-off, see Fig. 1.7a. Like many flow phenomena, pinch-off is strongly affected by the composition of the liquid system. Figure 1.7b shows a pinch-off event of a dilute polymer solution drop. The addition of a small amount of polymers fundamentally changes the shape of the liquid thread (c.f., Fig. 1.7a), which is now of almost uniform thickness along its entire length [38]. Dilute polymer solutions exhibit both viscous and elastic responses to deformation, due to stretching and subsequent relaxation of the polymer molecules present in the liquid, and thus have viscoelastic properties. In pinch-off, the polymers experience a diverging stress due their stretching by the extensional flow, resulting in an exponentially thinning thread, with minimum radius $r_{\min } \propto e^{-t /(3 \lambda)}$, where $\lambda$ is the relaxation time of the liquid (i.e., the timescale over which a stretched polymer returns to its equilibrium state) [38-40]. This striking example highlights the importance that each component (no matter how small or dilute) can have on the flow dynamics of a multi-component system, especially when singularities are present.

Coalescence (Fig. 1.6b), like break-up, has been an active field of research in recent years $[27,43-54]$. Figure 1.8a shows an example of the initial stage of 


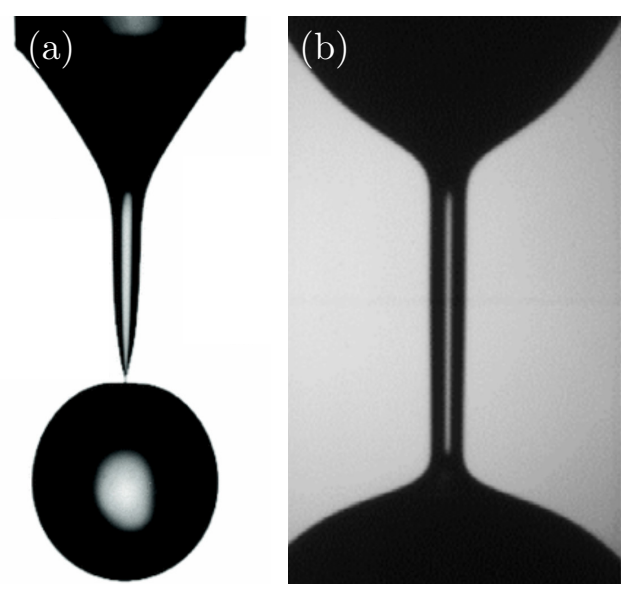

Figure 1.7: (a) Pinch-off of a water drop. Note the singularity at the connection of the thread to the drop. Image taken from [41]. (b) Pinch-off of a dilute polymer solution. A thin liquid thread of approximately uniform thickness forms. Image taken from [38].

coalescence, and reveals that a bridge that grows over time connects the two drops. The same singularity that is present at the end of pinch-off appears during coalescence, though in this case it appears at the start of the process, when the drops first come into contact and the bridge size is zero (Fig. 1.8a, at $t=0$ ). The dynamics of coalescence are typically expressed in terms of the width $w_{0}$ and height $h_{0}$ of the connecting bridge. One usually finds that these grow in time following a power law, i.e., $w_{0} \propto t^{\alpha}$ and $h_{0} \propto t^{\beta}$, where the values of the exponents $\alpha$ and $\beta$ depend primarily on the geometry and the viscosity of the drops. For example, it was found that $h_{0} \propto t$ for highly viscous sessile drops on a solid substrate with a small contact angle, whereas $h_{0} \propto t^{2 / 3}$ for their low-viscosity counterpart $[44,50,55,56]$. For spherical drops (whose coalescence dynamics are expressed in terms of the bridge radius $r_{0}$ due to axisymmetry) it was found that $r_{0} \propto t \ln t$ for low viscosity liquids, and $r_{0} \propto t^{1 / 2}$ for high viscosity liquids, with a crossover separating the two asymptotic regimes $[45,57-59]$. Apart from the temporal dynamics, the viscosity of the liquid also affects the shape of the connecting bridgecapillary waves, such as those shown in Fig. $1.8 \mathrm{~b}$, are universally present on coalescing drops with low viscosity, but are not observed for high viscosities $[43,47,50,56,59-62]$. 

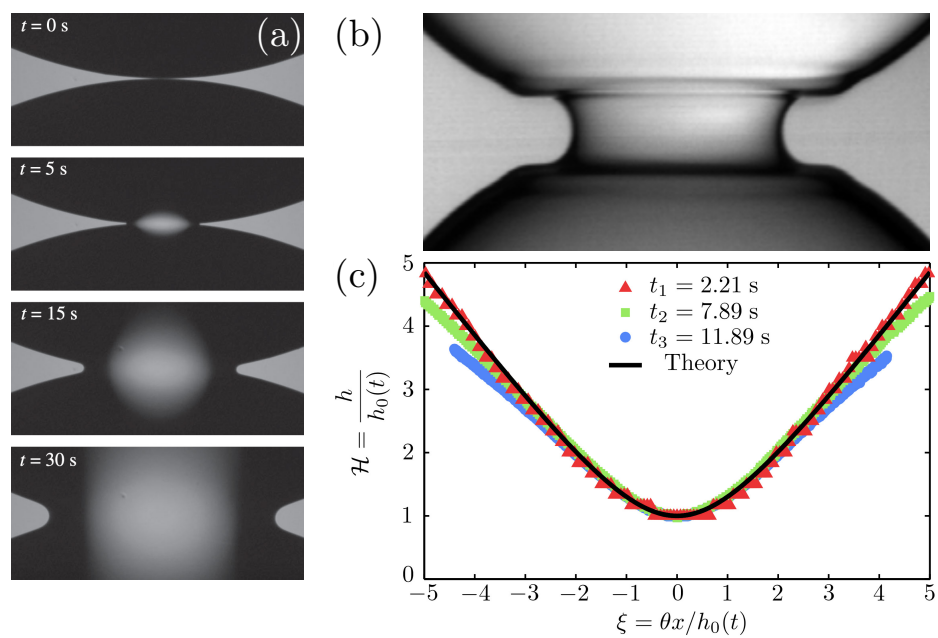

Figure 1.8: (a) Top-view of coalescing viscous drops on a solid substrate. Image taken from [42]. (b) Coalescence of low-viscosity spherical drops. Note the presence of capillary waves on the interface. Image taken from [43]. (c) Rescaled bridge profiles at different times for the coalescence of sessile viscous drops on a solid substrate. Collapse of the profiles indicates self-similar dynamics. The black line is the similarity solution of Eq. 1.2. Figure taken from [44].

Coalescence is by nature a three-dimensional problem. Nevertheless, it has been described successfully by two-dimensional models because the main flow direction in coalescing drops is towards the bridge $[42,44,57,59]$. Particular success for describing the dynamics of coalescing drops was achieved using self similarity-based approaches. Such methods assume that the growth dynamics of the bridge are determined by a small-scale region at the centre of the coalescing drops; the large-scale features of the drop (such as its shape far from the bridge and the outer contact lines) are assumed not to affect the bridge growth dynamics. This allows for considerable simplification of the Navier-Stokes equations. For sessile drops on solid substrates, for example, the coalescence dynamics in the viscous regime are then described by the thin-film equation

$$
\frac{\partial h}{\partial t}+\frac{\gamma}{3 \eta} \frac{\partial}{\partial x}\left(h^{3} \frac{\partial^{3} h}{\partial x^{3}}\right)=0,
$$

where $h(x, t)$ is the shape of the bridge, $t$ is the time, $\gamma$ is the surface tension, 
$\eta$ is the viscosity, and $x$ is the lateral coordinate. Equation 1.2 is valid only when the contact angle is small (i.e., when $\partial h / \partial x \ll 1$ ), and the typical length scale is smaller than $l_{c}$, such that gravity can be neglected. Furthermore, it assumes a no-slip boundary condition at the solid-liquid interface. Since the only relevant length scale in drop coalescence is the bridge height $h_{0}$, Eq. 1.2 has a similarity solution of the form

$$
h(x, t)=v t \mathcal{H}(\xi), \quad \text { with } \xi=\frac{\theta x}{v t},
$$

where $\mathcal{H}(\xi)$ is the similarity profile of the bridge, and $v$ is the coalescence velocity such that $h_{0}=v t$. Figure $1.8 \mathrm{c}$ shows experimentally obtained bridge profiles at different times scaled (both vertically and horizontally) according to Eq. 1.3; their collapse indicates the validity of the similarity solution [44]. Numerically solving Eq. 1.2 with the similarity solution Eq. 1.3 allows one to find $v=0.82 \gamma \theta^{4} /(3 \eta)$ and the bridge profile $\mathcal{H}(\xi)$, which is shown as the solid black line in Fig. 1.8c, in excellent agreement with the experiments [44]. A comparable self-similarity approach has been used to describe the coalescence of low viscosity drops [50]. This highlights the self-similar nature of coalescence, and that this feature can be exploited to theoretically predict the coalescence dynamics.

Many technological applications of drop coalescence involve liquids with complex properties. For example, the addition of polymers to ink gives it viscoelastic properties [65-67]. The effect of viscoelasticity on jet break-up is well-understood, and it has been shown to play a large role near the singularity that appears during pinch-off (Fig. 1.7) [38-40]. By contrast, the effect of viscoelasticity on the singularity that appears at the start of coalescence remains unexplored, even though large effects are expected due to the diverging stress.
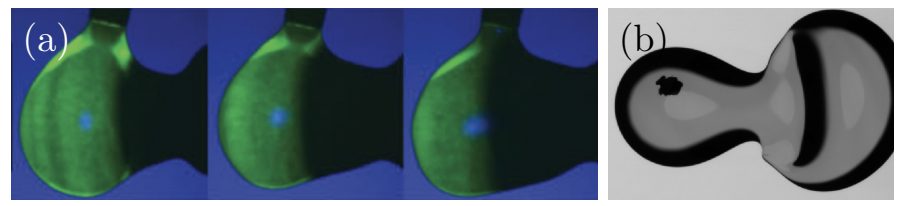

Figure 1.9: (a) When two drops of different surface tensions merge, liquid from the low surface tension drop (black) engulfs the high surface tension drop (green), imaged here using fluorescence. Image taken from [63]. Marangoni flow can delay the coalescence of sessile drops of different surface tensions. Image taken from [64]. 


\subsection{Fluid dynamics inspired by inkjet printing: a guide through the thesis}

Apart from viscoelastic effects, Marangoni flow can also present itself during coalescence. When two drops of different surface tensions merge, the drop with high surface tension is engulfed by the low surface tension liquid, to minimise the energetic cost of the drop interface, see Fig. 1.9a [63,68]. This fundamentally changes the coalescence dynamics - for example, Marangoni flow can hinder the coalescence of drops of different surface tensions in a process known as "delayed coalescence", which keeps the drops temporarily separated, see Fig. 1.9b [46, 49,52] A similar phenomenon was observed for the coalescence between drops with different surfactant concentrations [53]. Such phenomena are also expected to play a role in the coalescence of ink drops, since their complex composition gives rise to intricate dynamics.

\subsection{Fluid dynamics inspired by inkjet printing: a guide through the thesis}

The examples in the above sections illustrate that surface tension-driven multiphase flows are of both fundamental and industrial interest. Figure 1.10 gives an overview of several surface tension-driven flow phenomena that appear (and may lead to problems) in inkjet printing. These phenomena served as inspiration for the work presented in this thesis.
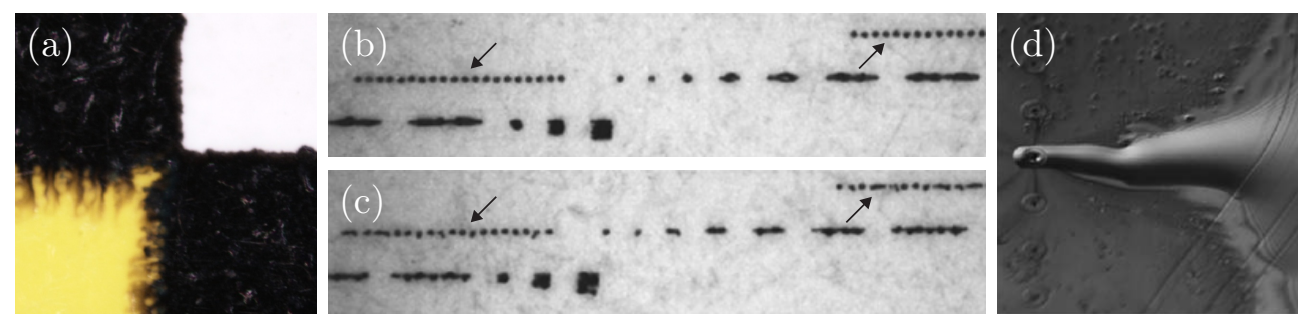

Figure 1.10: (a) Black ink flows into yellow ink due to the difference between their surface tensions, this is know as "bleeding". The fingering instability of the contact line leads to low print quality. (b) Ink drops deposited directly onto paper. (c) Ink drops deposited onto paper that was coated with a primer layer prior to ink deposition. Notice that the lines (indicated by the arrows) are not as straight as in (b). Images (a)-(c) courtesy of Canon Production Printing Netherlands B.V. (d) Ink on a nozzle plate. Marangoni flow drives the ink toward one of the nozzles. Image taken from [26]. 
Part I of this thesis deals with wetting phenomena. Consider the test print shown in Fig. 1.10a, where a patch of yellow ink is printed next to a patch of black ink. The interface between the different coloured inks is unstable, leading to fingering of the black ink into the yellow ink. This process is known as "bleeding". Printing on a salt-containing primer layer can prevent bleeding, but introduces additional complexity, since the substrate is now liquid instead of solid and the physico-chemical interaction between the salt and pigment needs to be taken into account [34]. The liquid nature of primer layers introduces new undesired effects - Figs. 1.10b-c show two test prints; the drop rows printed directly on paper (Fig. 1.10b) appear much straighter than those printed on liquid primer layers (Fig. 1.10c). The effect of primer layers on wetting dynamics is the focus of chapters 2 and 3 of this thesis.

Inspired by Figs. 1.10b-c, we experimentally study the mutual capillary interaction between drops on a thin liquid film in chapter 2. We show that the appearance of a visco-capillary wave on the surface of the liquid substrate plays an important role in mediating the interaction between drops.

We study the physico-chemical interaction between the primer layer and deposited ink drops in chapter 3 . The simultaneous effects of hydrodynamic flow and particle destabilisation result in intricate ring-shaped patterns, whose dynamics we quantify.

In chapter 4, we investigate the wetting properties of water-based solutions of alkane diols (surfactant-like liquids) on solid substrates. Alkane diols are commonly used in inks, despite their surprisingly complex wetting behaviour. The wetting properties of inks on solid (silicon-based) substrates are a crucial parameter for the reliable production of ink drops, since ink can wet the nozzle plate (see Fig. 1.10d), which has the potential to cause nozzle failure [26]. We experimentally reveal that the contact angle of drops consisting of such solutions is determined by two mechanisms - Marangoni contraction and autophobing. Both mechanisms result in unexpectedly large contact angles.

Part II of this thesis deals with coalescence. In chapters 5 and 6 , we continue our investigation of drop dynamics on primer layers, such as those shown in Figs. 1.10b-c, by studying the coalescence of drops on liquid substrates. These drops are known as "liquid lenses" [69]. Compared to the well-known case of coalescence on solid substrates, the coalescence dynamics of liquid lenses are relatively unexplored [35]. In chapter 5, we experimentally quantify the coalescence dynamics of liquid lenses for a wide range of viscosities. The bridge that connects the coalescing lenses is found to grow with self-similar dynamics - in both the viscous and inertial regimes. 
In chapter 6 , we further develop our understanding of the coalescence of liquid lenses, focusing on the dynamics in the viscous regime. The bridge dynamics converge toward a self-similar solution, though this convergence can be slow. We show that the fastest mode of coalescence involves a global translation of the lenses, which is not observed for the coalescence of viscous drops on a solid substrate.

Inspired by the viscoelastic properties of ink, we study the coalescence of viscoelastic drops in chapter 7 . The emergence of an elastic singularity is investigated using experimental methods and scaling analysis.

Finally, in chapter 8 , we study the mid-air coalescence of drops of different surface tensions. Using a combination of experiments and numerical simulations, we investigate the asymmetrical shape of these drops during their coalescence, with particular attention being paid to the role of capillary waves. 



\section{Part I}

\section{Wetting}





\section{Chapter 2}

\section{Printing wet-on-wet: Attraction and repulsion of drops on a viscous film}

Wet-on-wet printing is frequently used in inkjet printing for graphical and industrial applications, where substrates can be coated with a thin liquid film prior to ink drop deposition. Two drops placed close together are expected to interact via deformations of the thin viscous film, but the nature of these capillary interactions is unknown. In this chapter, we show that the interaction can be attractive or repulsive depending on the distance separating the two drops. The distance at which the interaction changes from attraction to repulsion is found to depend on the thickness of the film and increases over time. We reveal the origin of the non-monotonic interactions, which lies in the appearance of a visco-capillary wave on the thin film induced by the drops. Using the thin-film equation, we identify the scaling law for the spreading of the waves and demonstrate that this governs the range over which the interaction is observed.

\ Published as: Michiel. A. Hack, Maxime Costalonga, Tim Segers, Stefan Karpitschka, Herman Wijshoff, and Jacco H. Snoeijer, Printing wet-on-wet: Attraction and repulsion of drops on a viscous film, Appl. Phys. Lett. 113, 183701 (2018). 


\subsection{Introduction}

Solid particles at a liquid-gas interface have a tendency to form clusters due to capillarity-driven interactions. This phenomenon is known as the "Cheerios effect", named after the floating cereals that form clusters at the milk-air interface [70]. Manifestations of the Cheerios effect are also found in biology. For instance, mosquito eggs aggregate on the surface of a pond to form rafts [5]. Capillary interactions have also been observed between liquid drops and between solid particles on soft gels [71-73]. Soft gels are solids but share many properties, such as having a surface tension, with highly viscous liquids [74]. Capillary interactions are also relevant to technological applications, which range from self-assembly to drop condensation [75-79].

In an industrial setting, capillary driven drop interactions play an important role in inkjet printing. Substrates are frequently covered by a first layer of ink before a second ink layer is applied or can be coated with a thin liquid primer layer prior to ink deposition [8]. Such primer layers contain salts that destabilise the colloidal pigment particles and thereby increase their sedimentation rate, which enhances print quality [34]. Typically, the primer layer thickness is similar to the size of the ink drops, since both are deposited using a similar printhead [10]. We have observed interaction between ink drops deposited on such a primer layer, see Fig. 1.10b-c. However, the nature of the capillary interactions between drops deposited on a thin liquid film is still

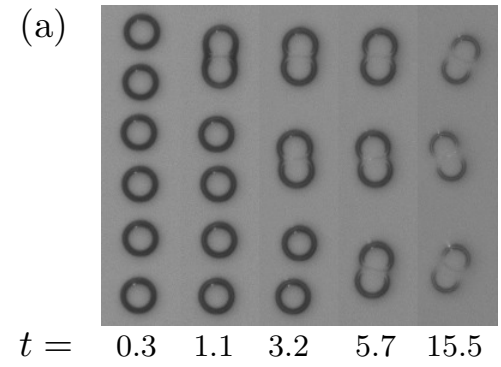

(b)

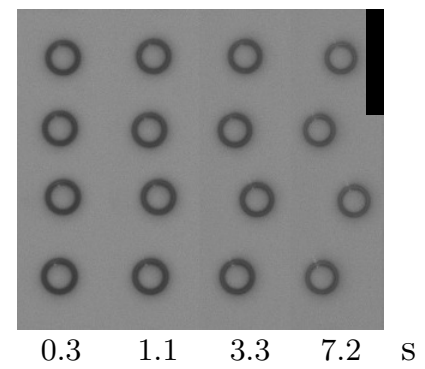

Figure 2.1: Interaction of water drops $(R=45 \mu \mathrm{m})$ printed on a thin silicone oil film $\left(h_{0}=5.7 \mu \mathrm{m}\right)$. Two types of interactions are observed: (a) Attractive interaction causes the drops to form drop pairs. (b) Repulsive interaction results in a zigzag-like pattern of drops. The difference between the two experiments is the initial distance between the drops in the printed line. The scale bar represents $400 \mu \mathrm{m}$. 


\subsection{Drop-induced surface deformation}

poorly understood.

In this chapter, we experimentally study capillary interactions between drops on thin liquid films. We focus on the case where the drops and films are immiscible, which eliminates mixing and Marangoni effects and isolates the Cheerios-like interactions. The essence of our experiment is shown in Fig. 2.1: a row of water drops (MilliQ, Millipore Corporation) with a radius of $R=45 \mu \mathrm{m}$ (which is the same in all experiments) is jetted onto a thin silicone oil film (Basildon Chemical Co. Ltd.) with a thickness $h_{0}=5.7 \mu \mathrm{m}$ and viscosity $\eta_{\mathrm{o}}=1 \mathrm{~Pa} \cdot \mathrm{s}$ using a piezo-driven pipette (AD-K-501, Microdrop Technologies). We observe both attractive (Fig. 2.1a) and repulsive (Fig. 2.1b) drop interactions, where the only difference between these experiments is the distance between the jetted drops. Attraction, as shown in Fig. 2.1a, results in drop pairs. The entrainment of a thin oil film between the drops delays their coalescence [80]. In Fig. 2.1b, by contrast, the drops are pushed out of the initially straight line, resulting in a zigzag-like configuration. The increase in drop distance clearly points to a repulsive interaction. The semi-coalesced drop pairs in Fig. 2.1a also show a zigzag structure at $t=15.5 \mathrm{~s}$, which indicates a possible repulsive interaction for $t>3.2 \mathrm{~s}$. Hence, we find that drops on thin viscous films exhibit intricate non-monotonic interactions.

\subsection{Drop-induced surface deformation}

The non-monotonic nature of the interactions can be traced back to the surface deformation induced by a single drop. In Fig. 2.2 we show the profile of the viscous film $\left(h_{0}=28 \mu \mathrm{m}\right)$ at various times after drop deposition, measured using digital holographic microscopy (abbreviated DHM, model R-1000, Lyncée Tec) [81]. The measured surface deformations are non-monotonic and extend over a distance of approximately $1 \mathrm{~mm}$, almost two orders of magnitude larger than the size of the drop. The wave-like profile results from volume conversation: the liquid is pulled up to create a meniscus close to the drop, and a capillary wave connects the meniscus to the flat film far away from the drop. The perturbed profile of the liquid film continues to broaden over time as is shown in Fig. 2.2a-d.

Since the interactions between drops are induced by perturbations of the viscous film, we expect the range of the interaction to increase over time. Such a time-dependence in the interaction law is fundamentally different from the usual Cheerios effect (particles at an interface of a deep pool) or the "inverted Cheerios effect" (drops or particles on elastic layers) [70-73, 82, 83]. 


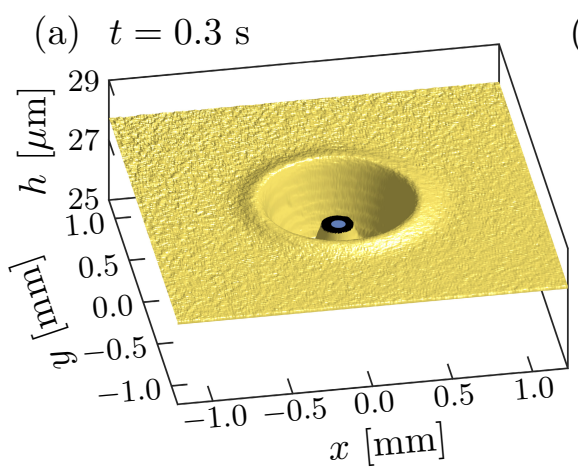

(b) $t=2.0 \mathrm{~s}$

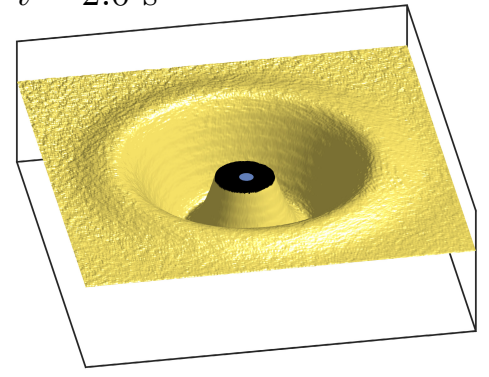

(c) $t=6.0 \mathrm{~s}$

(d) $t=10.1 \mathrm{~s}$
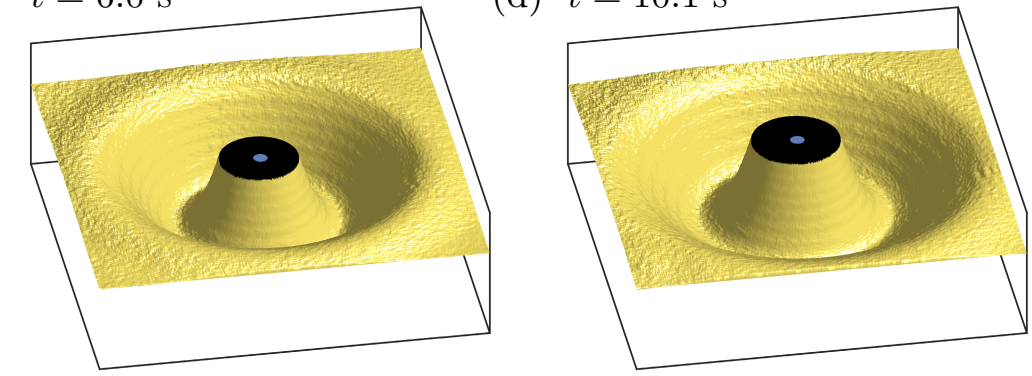

Figure 2.2: Evolution of the profile of the viscous film $\left(h_{0}=28 \mu \mathrm{m}\right)$ around a drop $(R=45 \mu \mathrm{m})$ located at the origin. The film exhibits a wavelike deformation that broadens over time: (a) $t=0.3 \mathrm{~s}$, (b) $t=2.0 \mathrm{~s}$, (c) $t=6.0 \mathrm{~s}$, and (d) $t=10.1 \mathrm{~s}$. The axes in (a) also apply to (b) $-(\mathrm{d})$. The black circle in the centre corresponds to a region where digital holographic microscopy cannot properly resolve the film's surface, and the blue circle denotes the drop's diameter and position in the $x y$ plane.

In those cases, the deformation by a single particle reaches a steady state, so the interaction law is constant over time. In the present case, by contrast, the time-scale of the change in the deformation of the film is similar to the timescale of the induced drop motion. This makes it very challenging to quantify the detailed interaction law. For this reason, we focus on finding the (timedependent) range of attractive interactions and correlate this to the evolution of the profile of the viscous film. 


\subsection{Interaction between two drops}

\subsection{Interaction between two drops}

To reveal how the interaction range depends on time and film thickness, we focus on the case of two drops, as sketched in the inset of Fig. 2.3a. Oil films of initially uniform thickness $h_{0}$ were spin-coated on a hydrophobic glass microscope slide (Menzel-Gläser). The thickness was varied by changing the rotational speed and spinning time of the spin-coater. The hydrophobisation, performed by vapour deposition of trichloro(octadecyl)silane (SigmaAldrich), resulted in a contact angle of water on glass sufficiently large to prevent rupture of the silicone oil film underneath the drops through "rewetting" [84]. The thickness of the silicone oil films was measured using reflectometry (HR2000+ spectrometer with HL-2000-FHSA halogen light source, Ocean Optics) [85]. The interfacial tension between the water drops, with surface tension $\gamma_{\mathrm{w}}=72 \mathrm{mN} / \mathrm{m}$, and the silicone oil film, with surface tension $\gamma_{\mathrm{o}}=21.2 \mathrm{mN} / \mathrm{m}$, was $\gamma_{\text {wo }} \approx 20 \mathrm{mN} / \mathrm{m}$. Consequently, since $\gamma_{\text {wo }}+\gamma_{\mathrm{o}}<\gamma_{\mathrm{w}}$, a thin silicone oil film engulfed the water drops [80,84,86,87]. The oil-coated glass substrate was mounted on a linear motor to control the distance between the drop centres $D$ through the speed of the substrate and the jetting frequency of the pipette. The time between the deposition of the two drops was typically around $10 \mathrm{~ms}$, which is much shorter than the relevant timescale over which the interaction is observed (from $t=0.28 \mathrm{~s}$ onwards). The deposited drops were imaged from below the substrate using a camera (Ximea XiQ MQ013MG-ON) connected to a telecentric lens (Kowa LM50TC), and the experiment was illuminated from above (Schott Ace light source with diffuser plate). The spatial and temporal resolution were $3.5 \mu \mathrm{m} /$ pixel and $20 \mathrm{~ms}$, respectively. The images were processed to extract the time-dependent drop positions from which the separation distance $D$ and the interaction type (i.e., attraction or repulsion) were determined.

From the experiments we determine the type of interaction between two drops. In Fig. 2.3, we show a typical series of experiments with $h_{0}=46 \mu \mathrm{m}$, varying the initial drop-drop separation distance $D$. In Fig. 2.3a, we report whether the interaction is attractive (denoted $\mathrm{A}$ in the figure) or repulsive (denoted $\mathrm{R}$ ) for varying distance $D$ and at various times $t$ after deposition. Here, each data point corresponds to one experiment with two drops. We observe a sharp transition between attractive interactions (small $D$ ) and repulsive interactions (large $D$ ), and we denote the range of attractive interaction by the critical separation $D^{*}$. We calculate $D^{*}$ by taking the mean of all $D$ values in the small region where attraction and repulsion overlap. The error in $D^{*}$ comprises of the standard deviation of these data points and the pixel error 

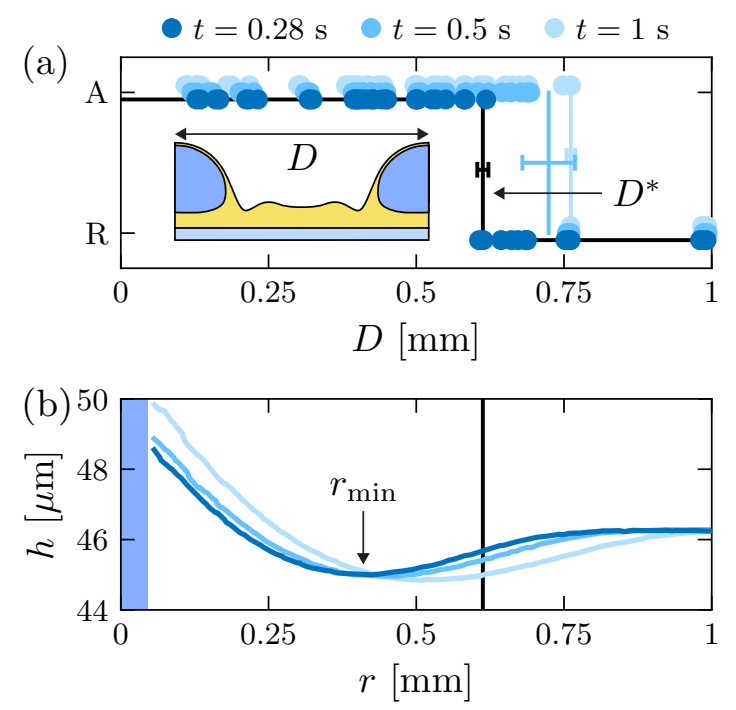

Figure 2.3: (a) Interaction type of two drops separated by a distance $D$ ( $A=$ attraction, $R=$ repulsion), at $t=0.28,0.5$, and $1 \mathrm{~s}$ (film thickness $\left.h_{0}=46 \mu \mathrm{m}\right)$. The distance $D$ is measured centre-to-centre (inset). The arrow indicates $D^{*}$ for $t=0.28 \mathrm{~s}$. The horizontal bars indicate the error in $D^{*}$. The datapoints are slightly vertically shifted for clarity. (b) The surface profile induced by a single drop (same conditions as panel (a)). The black line indicates the position of $D^{*}$ at $t=0.28 \mathrm{~s}$, while the arrow indicates $r_{\min }$. The blue region close to $r=0$ indicates the radius of the drop.

in $D$. The experiments show that $D^{*}$ is not a universal length, as it is observed to increase over time. Deformation of the drops, as observed from the top-view images, is small and occurs only when $D \ll D^{*}$ (i.e., in the case of attracting drops that are in very close proximity of each other), and thus does not affect the value of $D^{*}$. Note that the drops in Fig. 2.1 remain circular in shape, except when in close proximity. Here, we remark that $D^{*}$ is much larger than both $h_{0}$ and $R$-for example, we measure $D^{*}=0.61 \mathrm{~mm}$ at $t=0.28 \mathrm{~s}$. This observation is consistent with the behaviour observed for the printed drop rows in Fig. 2.1, where the drop spacing is also the key factor that determines the interaction type.

In a separate experiment, DHM was used to measure the drop-induced surface deformation of the oil film under the same conditions as for Fig. 2.3a. Figure 2.3b shows the surface profile of the oil film from the centre of the drop 


\subsection{Interaction between two drops}

outward along a radial line with coordinate $r$, where $r=0$ corresponds to the drop centre, at various times $t$. DHM is unable to measure the film profile close to the drop because the slope of the surface is too steep in this region (indicated by the black circle in Fig. 2.2). Comparison of Figs. 2.3a and 2.3b shows that $D^{*}$ is indeed directly comparable to the extent of the deformation of the film (approximately $0.1-1 \mathrm{~mm}$ ), demonstrating that the interaction is indeed governed by this deformation. Since the deformation of the film evolves over time, the nature of the drop-drop interaction is time-dependent as well.

Since the broadening of the surface deformations is expected to change with film thickness, we next study the dependence of $D^{*}$ on $h_{0}$. The experiments from Fig. 2.3a were repeated with oil films of varying $h_{0}$, and the results are shown in Fig. 2.4a. Clearly, the value of $D^{*}$ strongly depends on $h_{0}$. Indeed, this can be correlated to the dynamics of the deformed surface, which exhibits a similar dependence on $h_{0}$. To demonstrate this, we characterise the film deformation for various film thicknesses using DHM. The time-evolution of the position of the dimple $r_{\min }$, i.e., the first minimum in the profile as defined in Fig. 2.3b, is plotted in Fig. 2.4b for several film thicknesses. Clearly, the dynamics are strongly affected by the thickness of the film. This can be understood from the thin-film equation for the film profile $h(x, y)$, which reads

$$
\frac{\partial h}{\partial t}=-\frac{\gamma_{\mathrm{o}}}{3 \eta_{\mathrm{o}}} \nabla \cdot\left(h^{3} \nabla \nabla^{2} h\right) .
$$

The typical length-scale for the film thickness is $h_{0}$, while the gradient $\nabla$ acts along the lateral direction and is set by the radial distance to the drop $r$. With this, the terms in Eq. 2.1 are expected to scale as

$$
\frac{h_{0}}{t} \propto \frac{\gamma_{\mathrm{o}}}{\eta_{\mathrm{o}}}\left(\frac{h_{0}^{4}}{r^{4}}\right)
$$

such that

$$
r \propto\left(\frac{\gamma_{\mathrm{o}}}{\eta_{\mathrm{o}}} h_{0}^{3} t\right)^{1 / 4}
$$

Similar scaling laws have been observed for the flattening time of step-shaped thin polymer films, and for the wavelike deformation of a liquid close to a solid wall [88-90]. Figure 2.4c shows the same data as Fig. 2.4b, rescaled using the scaling from Eq. 2.3, i.e., $r_{\min } /\left(\gamma_{\mathrm{o}} h_{0}^{3} / \eta_{\mathrm{o}}\right)^{1 / 4}$. The data collapses onto a universal curve, in agreement with Eq. 2.3. 
(a)

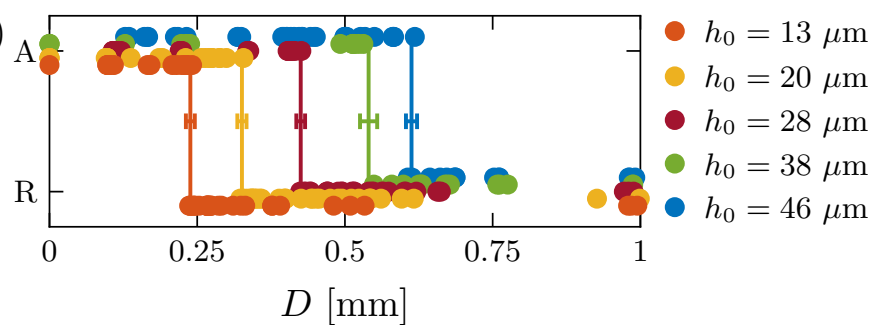

(b)

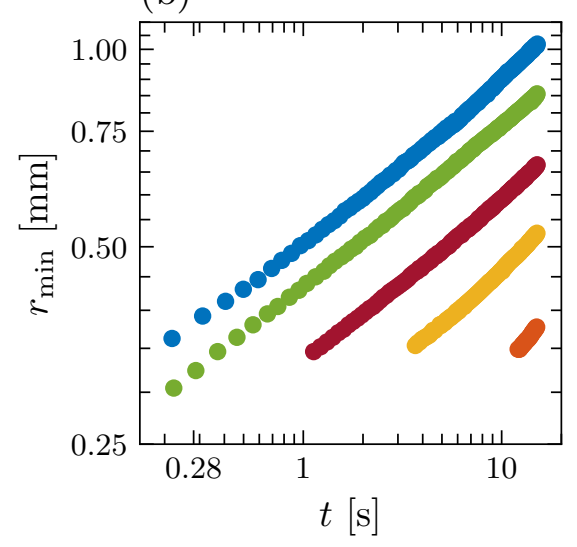

(c)

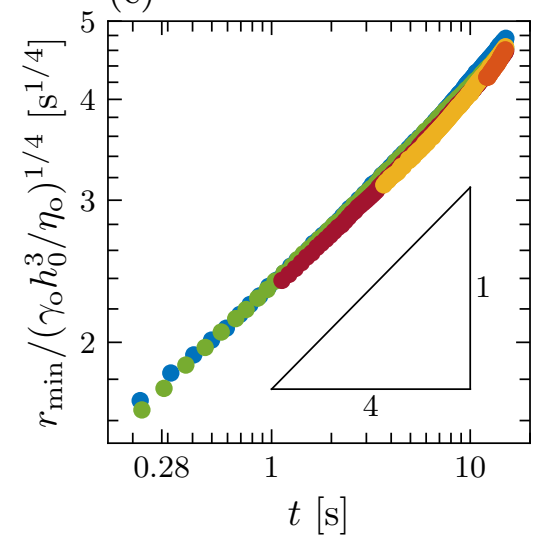

(d)

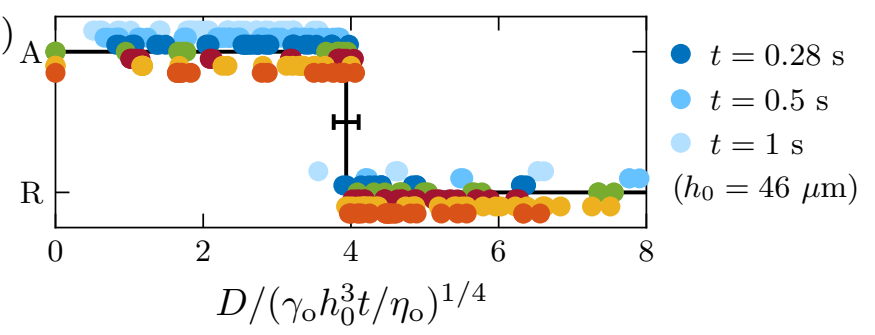

Figure 2.4: (a) The interaction type of two drops separated by a distance $D$ at $t=0.28 \mathrm{~s}$, for films of varying thickness $h_{0}$. The solid lines indicate $D^{*}$, with the horizontal bars indicating the error in $D^{*}$. (b) The position of the dimple $r_{\min }$ as a function of time. (c) The position of the dimple follows the scaling predicted by Eq. 2.3. (d) The same data as in (a) and Fig. 2.3a, but with $D$ scaled according to Eq. 2.3. The solid black line shows the normalised value of $D^{*}$, which takes on a single value; the horizontal bar indicates the standard deviation. 


\subsection{Interaction between two drops}
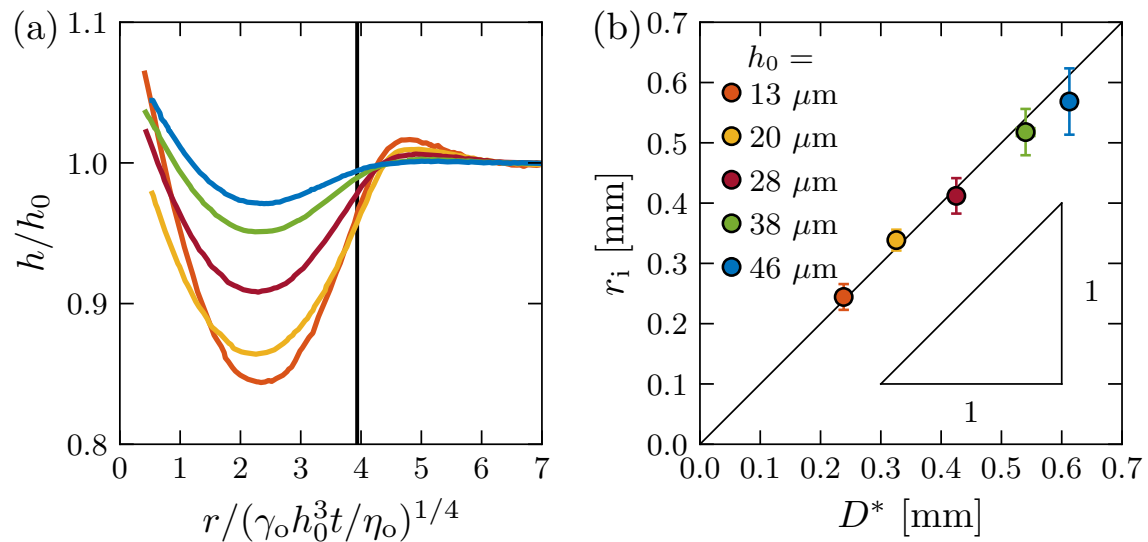

Figure 2.5: (a) The distance at which the interaction transitions from attraction to repulsion $D^{*}$ as a function of the location of the inflection point of the surface profile $r_{\mathrm{i}}$ at $\mathrm{t}=0.28 \mathrm{~s}$. The solid black line indicates $D^{*}=r_{\mathrm{i}}$ as a guide to the eye. (b) The surface profile close to the drop for various film thicknesses normalised by the scaling law in Eq. 2.3. The solid black line corresponds to the transition from Fig. 2.4c.

We now apply the same scaling law to quantify the range of interactions between two drops. Figure 2.4d shows the drop interaction type as a function of the drop spacing $D$ normalised using Eq. 2.3 for a range of film thicknesses. All data collapse on a single curve with the transition from attraction to repulsion at $D^{*} /\left(\gamma_{\mathrm{o}} h_{0}^{3} t / \eta_{\mathrm{o}}\right)^{1 / 4} \approx 4$. Thus, the drop-induced deformation of the surface of the thin liquid film is indeed at the origin of the interactions.

Finally, we wish to quantify what property of the deformation determines whether drops attract or repel. In the example shown in Fig. 2.3, the transition between attraction and repulsion $D^{*}$ coincides with the inflection point of the deformed surface $r_{\mathrm{i}} .{ }^{1}$ This is a general result, as can be seen from Fig. 2.5a, where profiles of films with varying thickness have been rescaled according to the lubrication prediction. Indeed, in all cases, the critical distance $D^{*} /\left(\gamma_{\mathrm{o}} h_{0}^{3} t / \eta_{\mathrm{o}}\right)^{1 / 4} \approx 4$ corresponds to the inflection point. This is

\footnotetext{
${ }^{1}$ We calculate $r_{\mathrm{i}}$ by finding the $r$-coordinate of the maximum of $\partial h / \partial r$, which corresponds to $\partial^{2} h / \partial r^{2}=0$. The error is determined by finding the two $r$-coordinates where $\partial h / \partial r$ is equal to $\partial h / \partial r-0.05 \cdot \partial h /\left.\partial r\right|_{r=r_{\mathrm{i}}}$, where the last term is typical of the noise in the surface profile. Since the surface profiles flatten with increasing thickness, whereas the noise remains unchanged with thickness, the error bars increase for increasing $r_{\mathrm{i}}$ in Fig. 2.5.
} 
further quantified in Fig. 2.5b, showing the direct correspondence of $D^{*}$ and $r_{\mathrm{i}}{ }^{2}$ Thus, we conclude that the interaction is determined by curvature of the viscous film. Intriguingly, this result appears to be different from the interaction between drops as observed on an elastic medium [71,72]. In that case, the transition from attraction to repulsion was found to depend on whether the separation distance $D$ was small or large compared to the size of the drop $R$. For the case considered here, for which $D \gg R$, the elastic interaction can be described by a potential $\sim \nabla^{2} h$, and the change from attraction to repulsion occurs when the potential has a maximum [83]. Yet, in our experiments we find $D^{*}$ to occur when $\nabla^{2} h \approx 0$ (i.e., not at its maximum), for reasons that remain to be identified. We emphasise once more that for the elastic case the interaction law does not change over time, while, by contrast, the viscous film evolves dynamically. These dynamics bring along additional viscous forces that may be the cause for the unexpected role of the inflection point of the profile.

\subsection{Conclusion and outlook}

In conclusion, we have observed non-monotonic capillary interactions between liquid drops on thin liquid films, focusing on the case of immiscible liquids. These non-monotonic interactions are due to visco-capillary waves on the viscous films, induced by the presence of the drops on the film. The range of the interaction increases with time, due to the broadening of the waves, which makes this "viscous Cheerios effect" very different from the interactions observed on deep pools or on elastic substrates. Additionally, we have shown that the transition from attraction to repulsion coincides with the inflection point of the deformed surface.

These results will be of importance for inkjet printing whenever drops are deposited on primer layers: capillary waves are also observed when drops are miscible, though in that case other factors such as mixing and Marangoni flows are expected to play a role. Surface-mediated drop interactions that appear due to the presence of a primer layer could be an explanation for the quality difference between the example prints shown in Figs. 1.10b-c,

\footnotetext{
${ }^{2}$ Due to the limited ability of DHM to resolve areas of the surface with high slopes we were unable to measure the deformation of the film at $t=0.28 \mathrm{~s}$ for the three thinnest films $\left(h_{0}=\right.$ $13,20,28 \mu \mathrm{m})$. We therefore measured at a time $t_{\text {measure }}>0.28 \mathrm{~s}$, and extrapolated back to $t=0.28 \mathrm{~s}$ using the scaling law from Eq. 2.3 (in the form $\left.r(t)=\left(t / t_{\text {measure }}\right)^{1 / 4} r\left(t_{\text {measure }}\right)\right)$ to obtain the value of $r_{\mathrm{i}}$ at $t=0.28 \mathrm{~s}$. Measurements of the films with $h_{0}=38$ and $46 \mu \mathrm{m}$ were performed at $t=0.28 \mathrm{~s}$ and required no extrapolation.
} 


\subsection{Conclusion and outlook}

where the drop line that is printed on a primer layer (Fig. 1.10c) is not as straight as the drop line that is printed directly onto paper (Fig. 1.10b). More generally, drop interactions on thin films might be of use for applications such as anti-fouling and self-assembly. For example, substrates could be fine-tuned for fog harvesting, such that the interactions between drops lead to faster condensation of water [79]. 



\section{Chapter 3}

\section{Ring-shaped colloidal patterns on thin saline water films}

In this chapter, we reveal that a colloidal drop forms a ring-shaped pattern when deposited on a thin saline water film. Electrostatically stabilised colloidal particles in the drop destabilise when brought into contact with cations from the saline water film, such that they form particle clusters. We experimentally demonstrate that this physico-chemical process, when combined with hydrodynamic flow, can cause particle clusters to aggregate in an intricate ring-shaped pattern. We show that the shape of the pattern is largely determined by an undulation of the contact line that appears upon first contact of the drop with the liquid film. Finally, we reveal that the clusters that constitute the ring are transported radially outwards by Marangoni flow.

@ To be submitted as: Michiel A. Hack, Marjolein van der Linden, Herman Wijshoff, Jacco H. Snoeijer, Tim Segers, Ring-shaped colloidal patterns on thin saline water films. 


\subsection{Introduction}

Multi-component flows are ubiquitous in technological applications and nature alike. For example, multi-component liquids are often used in the context of patterning, and the presence of salt in oceans leads to large-scale complex flow structures $[91,92]$. The description of such flows is typically not complete without taking the physico-chemical interactions into account that occur between the separate components [14]. A technology where a strong coupling between hydrodynamic flow and physico-chemical processes exists is inkjet printing - a technique that allows the controlled deposition of liquid materials on a substrate $[7,8]$. Capillary flow phenomena are present throughout the entire inkjet printing process - small ink drops are generated by the RayleighPlateau instability, ink can spread on printing nozzles through Marangoni flow, and ink is absorbed by the paper substrate through capillary action $[11,12,26]$. Furthermore, ink contains a multitude of components, such as surfactants, pigments, polymers, and viscosity modifiers [13]. Each of these can affect the flow dynamics of ink - the presence of colloidal particles and surfactants in sessile drops leads to intricate drying dynamics, and the presence of polymers significantly alters the jetting behaviour of ink $[8,9,30,32,66]$.

One of the key challenges in inkjet printing is to obtain accurate control of the deposition of pigment (colloidal particles that provide colour to ink) on a substrate. This is particularly complicated by the fact that different stages of the inkjet printing process require different ink properties for optimal performance [7]. For example, pigment particles need to be stabilised (i.e., clustering needs to be prevented) and need to be small enough to be able to jet the ink, since bubbles may nucleate on large particles, resulting in jetting failure [93]. On the contrary, large pigment particles are desired for bright colours, since small pigment particles follow the flow of the carrier liquid into porous substrates (such as paper) resulting in pale colours [30,94]. A similar dichotomy appears for the evaporation properties of ink - slow evaporation of ink is desired at the nozzle, since the nozzle may get clogged if the particle concentration increases due to fast evaporation. On the substrate, however, slow evaporation can result in colour-to-colour bleeding (see Fig. 1.10a) when different coloured ink drops merge, which significantly lowers print quality. Additionally, fast evaporation is also required for further handling of the printed paper, to prevent ink smudging.

A solution to the above challenges is to cover the substrate with a coating called a "primer layer" before printing the ink layer [8]. Such primer layers typically consist of a salt dissolved in water [34]. Inks are, at their most 


\subsection{Introduction}

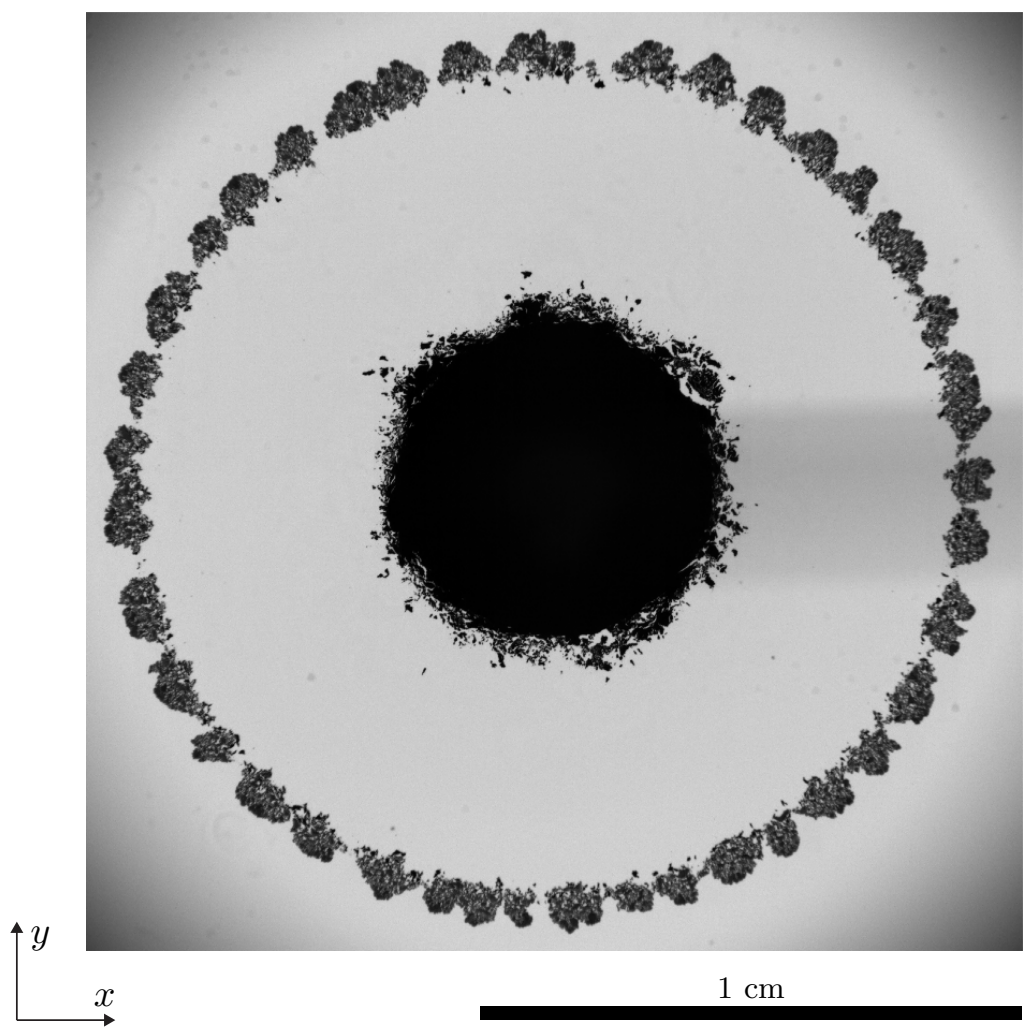

Figure 3.1: A typical ring-shaped pattern that appears upon the deposition of a colloidal drop on a thin liquid film consisting of $\mathrm{CaCl}_{2}$ (a salt) dissolved in water (top view). The dark unfocused line extending from the drop in the centre to the rightmost side of the image is the shadow of the needle used to deposit the drop.

basic description, stable suspensions of pigment particles. Stability can be induced by, for example, attaching negatively charged molecular groups to the pigment particles to induce suspension stability by electrostatic means [13]. The interaction between two pigment particles is then described by the DLVO theory, which states that the particles experience two types of interactions: a repulsive electrostatic interaction at relatively large distance, and a Van der Waals attraction at close range $[95,96]$. The pigment particles do not have sufficient energy to overcome the energy barrier that the negatively charged molecular groups induce, hence the pigment suspension is stable. The purpose 
of the cations in the primer layer is to lower the energy barrier between pigment particles upon first contact between the ink and primer layer [13]. In doing so, the particles are destabilised (since they can now overcome the energy barrier) and form clusters that are too large to follow the carrier liquid into the porous structure of the substrate (such that the colours remain bright), or into neighbouring ink drops (such that no bleeding occurs), resulting in better print quality [34,97].

In this chapter, we study a model system that resembles the deposition of an ink drop on a primer layer. In particular, we reveal that the combined effects of hydrodynamic flow and colloidal destabilisation can lead to intricate ring-shaped patterns. An example of such a pattern in presented in Fig. 3.1, which shows the outcome of the deposition of a (millimetre-sized) colloidal drop on a liquid substrate consisting of calcium chloride dissolved in water (several micrometres thick). The drop leaves a large dense blot in the centre of the image, and is surrounded by a ring-shaped pattern of clustered particles.

The chapter is structured as follows. In section 3.2 we describe the experimental methods. A general overview of the ring-shaped patterns in given in section 3.3. In section 3.4 we quantify the dynamics of the patterns, and in section 3.5 we propose a mechanism for the formation of the observed patterns. We end with our conclusion and outlook in section 3.6.

\subsection{Experimental methods}

\subsubsection{Colloidal suspensions}

We used carbon black pigment (Cab-O-Jet 400, CABOT Corporation) as colloidal particles. These particles are functionalised with geminal bisphosphonic acid groups that stabilise the suspension by means of electrostatic repulsion, and their diameter is approximately $130 \mathrm{~nm}[98,99]$. We controlled the mass concentration $c_{\mathrm{d}}$ of the colloids in the suspension through dilution of the stock suspension $\left(c_{\mathrm{d} \text {,stock }}=15 \mathrm{wt} \%\right.$ ) with water (MilliQ, Millipore Corporation). The surface tension of the suspensions was measured using the pendant drop method, and was found to be independent of the particle concentration, see Fig. $3.2[100]$.

\subsubsection{Thin saline water films}

Salt solutions were prepared by dissolving calcium chloride $\left(\mathrm{CaCl}_{2}\right.$, SigmaAldrich) in water. The solutions were vigorously shaken until they were uni- 


\subsection{Experimental methods}

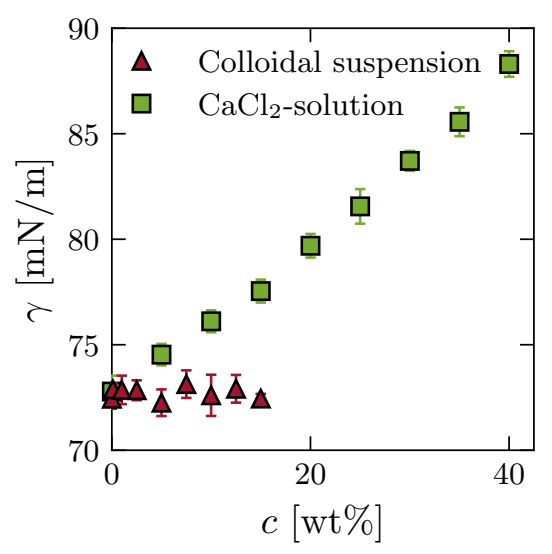

Figure 3.2: Surface tension $(\gamma)$ of the colloidal suspension and the $\mathrm{CaCl}_{2^{-}}$ solution as a function of their mass concentrations $(c)$ as measured by the pendant drop method. Every data point corresponds to an average of ten measurements, each of a fresh drop. The error bars represent the standard deviations of the ten measurements. We recorded 200 frames over a duration of two seconds for each drop.

formly transparent, and were allowed to cool down to room temperature before use. Glass microscope slides $(76 \times 26 \mathrm{~mm}$, Menzel-Gläser $)$ were used as base substrates for the thin liquid films. These were cleaned with acetone and water, blow-dried with nitrogren, and finally treated with plasma (PDC-002, Harrick Plasma) to induce hydrophilicity before use. Uniform films were obtained by spin-coating the glass substrates with the $\mathrm{CaCl}_{2}$-solutions. The film thickness was controlled through the spinning time of the spin-coater, and was measured for each film using reflectometry (HR2000+ spectrometer with HL2000-FHSA halogen light source, Ocean Optics). We measured the thickness of each film at five different spots to confirm uniformity of the film. The refractive index (used for the thickness measurements) and viscosity were taken from existing literature $[101,102]$. The surface tension of $\mathrm{CaCl}_{2}$-solutions increases significantly with the $\mathrm{CaCl}_{2}$ mass concentration $c_{\mathrm{f}}$ due to the ionic nature of $\mathrm{CaCl}_{2}$-solutions, see Fig. 3.2. The density of the $\mathrm{CaCl}_{2}$-solution, which was used to determine the surface tension, was measured using a densimeter (DMA 35, Anton Paar). We have verified that the film thickness (and thus the composition) remains approximately constant over time, and is not strongly affected by evaporation of the liquid, see appendix 3.7.1. 


\subsubsection{Experimental procedure}

The experiment consists of depositing a colloidal drop (volume $V=5 \mu \mathrm{L}$ ) on a thin saline water film and observing the subsequent pattern formation. The experimental setup is shown in Fig. 3.3. Two high-speed cameras were used to simultaneously record the dynamics from the top (Nova S12 camera, Photron with LM50TC lens, Kowa) and side view (Nova S12 camera, Photron with $12 X$ zoom lens, Navitar) perspectives at 10000 frames per second. The top view perspective was used to observe the pattern formation process, while the side view perspective was used to accurately determine the moment of first contact between the drop and the film's surface, and to measure the initial spreading dynamics of the drop. Two light sources were used to illuminate the experiment from the side (KL2500, Schott) and below (uniform LED backlight, Phlox). A different bottom lighting technique using a divergent light source (KL2500, Schott) was used for several experiments to image the curvature of the liquid-air interface of the thin film. The drops were generated on a

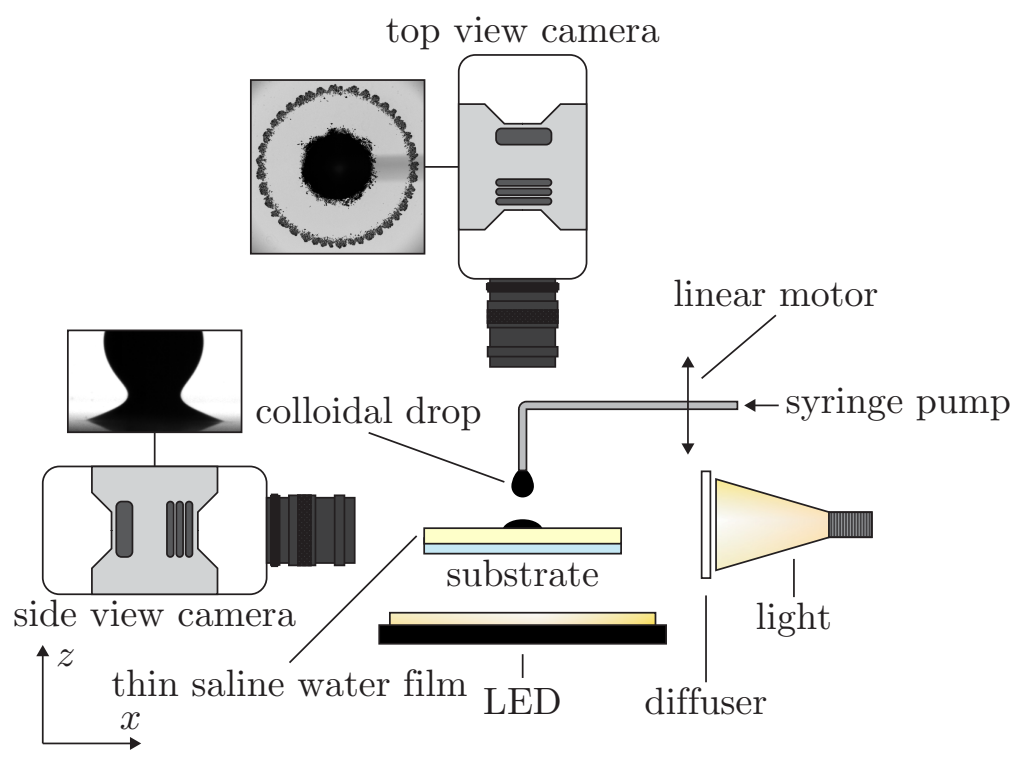

Figure 3.3: Schematic of the experimental setup. A (millimetre-sized) colloidal drop is deposited on a saline water film (several micrometres thick), and its subsequent dynamics are recorded from the top and side view using two high-speed cameras. 


\subsection{Ring-shaped patterns}

needle (26 gauge, Hamilton Company) by a syringe pump (PHD2000, Harvard Apparatus). To ensure reproducible and gentle deposition of the drops, the needle was moved towards the surface of the thin film by a programmable linear motor (T-LSR150B, Zaber) with a velocity $(\approx 0.01 \mathrm{~m} / \mathrm{s})$ that was much smaller than the typical contact line velocity during the initial spreading phase $(\approx 0.42 \mathrm{~m} / \mathrm{s})$.

\subsection{Ring-shaped patterns}

We first provide a general description of the observed patterns, and determine how their qualitative features depend on the properties of the colloidal drop and the saline water film. Figure 3.4 shows the formation of a pattern for typical material properties $\left(c_{\mathrm{d}}=10 \mathrm{wt} \%, c_{\mathrm{f}}=30 \mathrm{wt} \%, h_{0}=20 \mu \mathrm{m}\right)$. When the drop first touches the film (at $t=0$ ) it rapidly starts spreading. The contact line develops an undulate shape during this initial spreading motion (visible at $t=0.01 \mathrm{~s}$ ). At the same time, colloidal particles are destabilised by the presence of $\mathrm{Ca}^{2+}$ cations in the liquid substrate, resulting in the clustering of particles. These particle clusters are observed in Fig. 3.4 for $t \geq 0.02 \mathrm{~s}$. Some particle clusters separate from the contact line of the centre drop when it stops spreading (at $t \approx 0.02 \mathrm{~s}$ ), giving rise to the ring-shaped pattern. Interestingly, the outer particle clusters in the ring move at a lower radial velocity than the inner particle clusters. This means that small particle clusters in the ring merge, and that the ring width decreases over time (c.f., $t=0.15 \mathrm{~s}$ and $t=2.00 \mathrm{~s}$ ). The radius of the ring (and thus its perimeter) grows over time. This eventually forces the ring to break up into separate large clusters, since the network of clusters that constitutes the ring has finite extensibility. This is observed at $t=1.00 \mathrm{~s}$, where gaps have appeared in the ring, and $t=2.00 \mathrm{~s}$ where additional gaps have formed. Unsurprisingly, the gaps appear where the ring width is thinnest, i.e., at the weakest points of the ring with the smallest amount of contact between clusters. Finally, at large times $(t \geq 2.00 \mathrm{~s})$, the particle cluster morphology does not undergo major changes, and the clusters exhibit only radially outward motion.

The example shown in Fig. 3.4 indicates that the ring-shaped patterns likely depend on three features of the system, namely: (i) the presence of colloidal particles, (ii) a mechanism to destabilise these particles, and (iii) hydrodynamic flow in the drop and the liquid substrate. We therefore systematically vary the properties of the colloidal drop and the liquid substrate to determine the influence of each of these features on the ring-shaped patterns. 


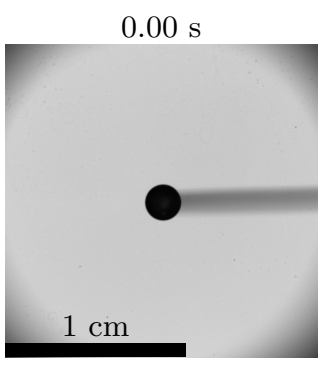

$0.15 \mathrm{~s}$

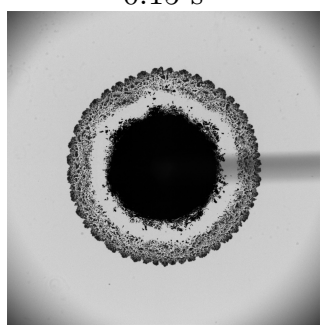

$0.01 \mathrm{~s}$

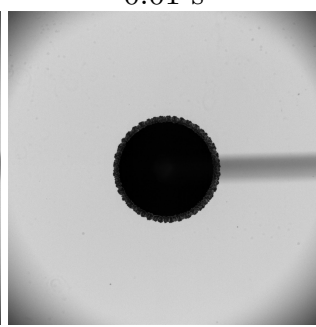

$0.50 \mathrm{~s}$

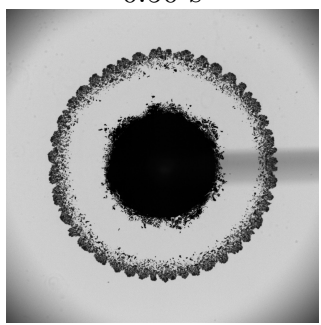

$0.02 \mathrm{~s}$

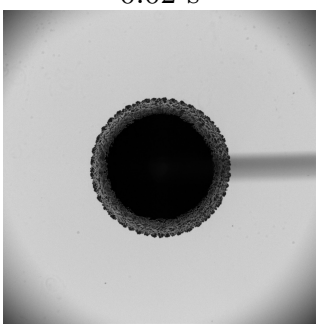

$1.00 \mathrm{~s}$

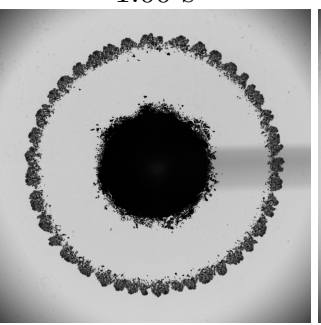

$0.04 \mathrm{~s}$
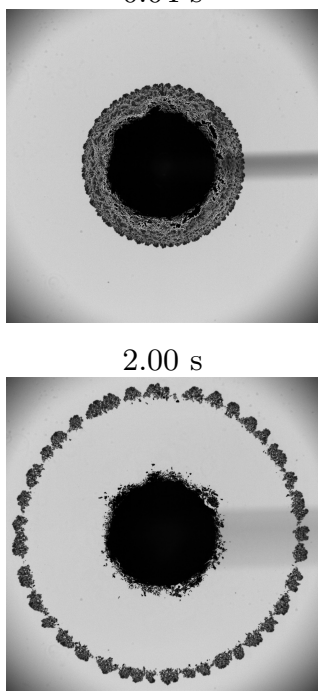

Figure 3.4: Image sequence showing the deposition of a colloidal drop $\left(c_{\mathrm{d}}=10 \mathrm{wt} \%\right)$ on a $c_{\mathrm{f}}=30 \mathrm{wt} \%$ film with $h_{0}=20 \mu \mathrm{m}$, and subsequent pattern formation.

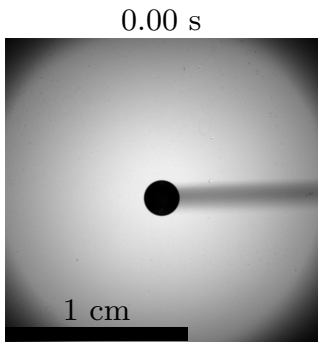

$0.15 \mathrm{~s}$

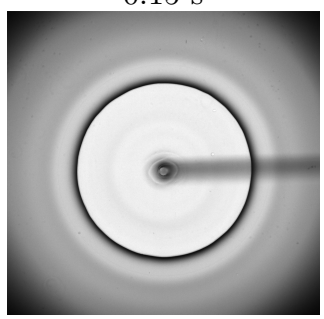

$0.01 \mathrm{~s}$

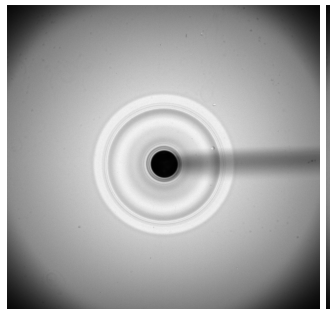

$0.50 \mathrm{~s}$

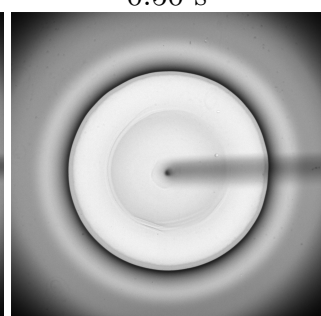

$0.02 \mathrm{~s}$

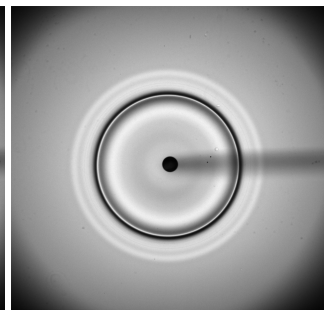

$1.00 \mathrm{~s}$

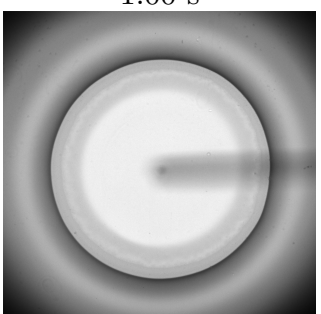

$0.04 \mathrm{~s}$

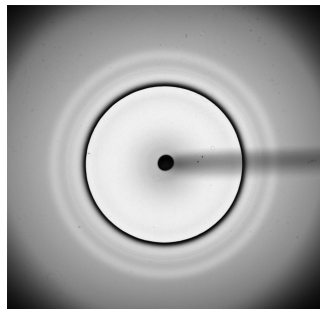

$2.00 \mathrm{~s}$

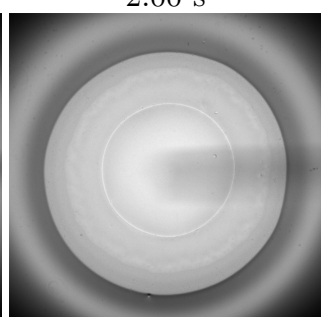

Figure 3.5: Image sequence showing the deposition of a water drop $\left(c_{\mathrm{d}}=0\right)$ on a $c_{\mathrm{f}}=30 \mathrm{wt} \%$ film with $h_{0}=20 \mu \mathrm{m}$. No contact line undulation is observed. 


\subsection{Ring-shaped patterns}

\subsubsection{Influence of the particle concentration}

We start by verifying whether the presence of colloidal particles is strictly required for the appearance of ring-shaped patterns, or whether the particles simply make the pattern visible. Figure 3.5 shows an experiment under the same conditions as the experiment shown in Fig. 3.4, but without colloidal particles $\left(c_{\mathrm{d}}=0\right)$. We used a divergent backlight (KL2500, Schott) for this experiment instead of the uniform (non-diverging) backlight that was used for the experiment shown in Fig. 3.4. This illumination technique allows us to visualise the curvature of the thin film's surface from changes in the image intensity. We observe no undulation of the contact line in the experiment shown in Fig. 3.5. We do, however, observe radial spreading dynamics (which we further quantify in section 3.4) similar to that of the ring in Fig. 3.4. While a ring-like shape is observed, we note that the undulate shape that is observed in the presence of particles is not present in Fig. 3.5. The presence of colloidal particles is thus crucial for the appearance of the pattern, and for that reason we investigate the influence of the particle concentration for $c_{\mathrm{d}}>0$.

In Fig. 3.6 we show patterns obtained for various $c_{\mathrm{d}}>0$ at $t=1.00 \mathrm{~s}$ while fixing the other parameters $c_{\mathrm{f}}=30 \mathrm{wt} \%$ and $h_{0} \approx 9 \mu \mathrm{m}$. For very small $c_{\mathrm{d}}$, such as $c_{\mathrm{d}}=0.1 \mathrm{wt} \%$ (Fig. 3.6a), the ring-shaped pattern is absent. The colloidal suspension is presumably too dilute at such small $c_{\mathrm{d}}$ for the particles to quickly come into contact with each other upon destabilisation by $\mathrm{Ca}^{2+}$ cations, such that the clusters that make up the ring are not formed. While some clustering is visible in Fig. 3.6a, these clusters are very sparsely

(a) $0.1 \mathrm{wt} \%$

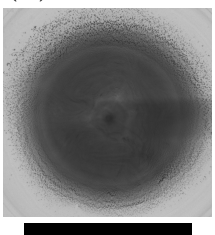

(b) $1.0 \mathrm{wt} \%$

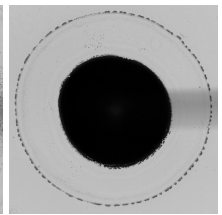

(c) $2.5 \mathrm{wt} \%$

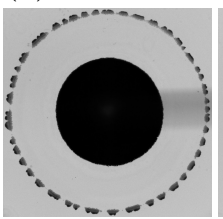

(d) $5.0 \mathrm{wt} \%$

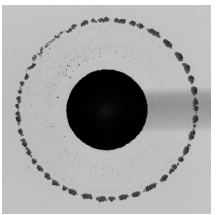

(e) $7.5 \mathrm{wt} \%$

(f) $12.5 \mathrm{wt} \%$

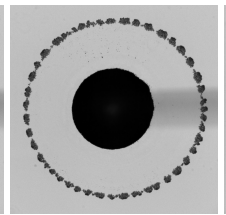

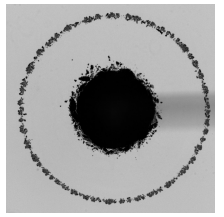

$1 \mathrm{~cm}$

Figure 3.6: Patterns observed for (a) $c_{\mathrm{d}}=0.1 \mathrm{wt} \%$, (b) $c_{\mathrm{d}}=1.0 \mathrm{wt} \%$, (c) $c_{\mathrm{d}}=2.5 \mathrm{wt} \%$, (d) $c_{\mathrm{d}}=5.0 \mathrm{wt} \%,(\mathrm{e}) c_{\mathrm{d}}=7.5 \mathrm{wt} \%$, and (f) $c_{\mathrm{d}}=12.5 \mathrm{wt} \%$. For all cases $t=1.00 \mathrm{~s}, c_{\mathrm{f}}=30 \mathrm{wt} \%$, and $h_{0} \approx 9 \mu \mathrm{m}$. The scale bar applies to all images. The ring-shaped pattern only appears for sufficiently high $c_{\mathrm{d}}$. 
distributed. The ring-shaped pattern is universally present for $c_{\mathrm{d}} \geq 1.0 \mathrm{wt} \%$, see Fig. 3.6b-f. The qualitative features of the patterns in Fig. 3.6b-f are mostly independent of $c_{\mathrm{d}}$; for example, we find that the ring width (a prominent feature of the patterns) does not increase with $c_{\mathrm{d}}$ for $c_{\mathrm{d}} \geq 2.5 \mathrm{wt} \%$. We hypothesise that, at such high $c_{\mathrm{d}}$, the colloidal particles are so densely distributed in the liquid that their destabilisation and clustering occurs on roughly the same time scale for all $c_{\mathrm{d}}$. We note that the fluid dynamical properties of the drop (e.g., surface tension and viscosity) are mostly independent of $c_{\mathrm{d}}$ (only $\rho$ shows a small dependence on $c_{\mathrm{d}}$ ), such that the difference in the pattern morphologies in Fig. 3.6 is the direct result of the particle destabilisation, and not hydrodynamics. In short, Figs. 3.4-3.6 reveal that a sufficiently high $c_{\mathrm{d}}$, and the appearance of the contact line undulation are both required for the ring-shaped pattern to appear.

\subsubsection{Influence of the $\mathrm{CaCl}_{2}$ concentration and film thickness}

We now turn to the role of the thin saline water films. The particle destabilisation rate depends on both the particle concentration as well as the $\mathrm{CaCl}_{2}$ concentration $c_{\mathrm{f}}$. We therefore expect significant effects of $c_{\mathrm{f}}$ if the pattern formation is (partly) governed by particle destabilisation, and not only by hydrodynamics. Figure 3.7 shows a series of experiments in which we varied $c_{\mathrm{f}}$ while keeping $c_{\mathrm{d}}=10 \mathrm{wt} \%$ fixed. When $c_{\mathrm{f}}=0$ (i.e., when the substrate is pure water), we observe neither an undulation of the contact line nor a ring-like pattern (Fig. 3.7a). Instead, the drop spreads out uniformly, without the visible formation of particle clusters at any point in the spreading process. This

(a) $0 \mathrm{wt} \%$

(b) $0.01 \mathrm{wt} \%$ (c) $0.1 \mathrm{wt} \%$

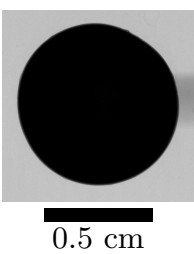

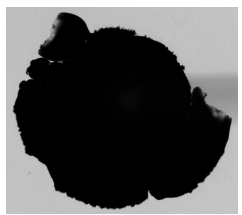

(d) $1 \mathrm{wt} \%$

(e) $5 \mathrm{wt} \%$

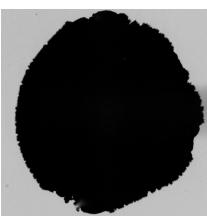

(f) $10 \mathrm{wt} \%$

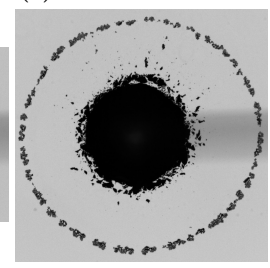

Figure 3.7: Patterns observed for (a) $c_{\mathrm{f}}=0 \mathrm{wt} \%$, (b) $c_{\mathrm{f}}=0.01 \mathrm{wt} \%$, (c) $c_{\mathrm{f}}=0.1 \mathrm{wt} \%,(\mathrm{~d}) c_{\mathrm{f}}=1 \mathrm{wt} \%$, (e) $c_{\mathrm{f}}=5 \mathrm{wt} \%$, and (f) $c_{\mathrm{f}}=10 \mathrm{wt} \%$. For all cases $t=1.00 \mathrm{~s}, c_{\mathrm{d}}=10 \mathrm{wt} \%$, and $h_{0} \approx 9 \mu \mathrm{m}$. The scale bar applies to all images. The ring-shaped pattern only appears for sufficiently high $c_{\mathrm{f}}$. 


\subsection{Ring-shaped patterns}

is additional evidence for the importance of particle destabilisation for the formation of the ring-like patterns.

Inspired by this result, we increase $c_{\mathrm{f}}$ in small steps in Fig. 3.7b-f. Surprisingly, even a very small amount of $\mathrm{CaCl}_{2}$ has a strong effect on the morphology of the contact line - a deformation of the contact line (though non-periodic) can be observed for concentrations as low as $c_{\mathrm{f}}=0.01 \mathrm{wt} \%$. Further increase of $c_{\mathrm{f}}$ shows no significant effect (Fig. 3.7c-d) until $c_{\mathrm{f}} \geq 5 \mathrm{wt} \%$ (Fig. 3.7e), where clusters that separate from the centre drop are first observed. These clusters, however, are not transported far away from the centre drop, such that the ring-shaped pattern is still absent. For $c_{\mathrm{f}}=10 \mathrm{wt} \%$ (Fig. 3.7f) and higher (Fig. 3.4) the ring-shaped pattern appears, and we note that (similar to $c_{\mathrm{d}}$ ) the qualitative features of the ring do not strongly depend on $c_{\mathrm{f}}$ for $c_{\mathrm{f}} \geq 5 \mathrm{wt} \%$.

We note that the surface tension (see Fig. 3.2) and viscosity of the film both depend on $c_{\mathrm{f}}$, such that we cannot fully exclude hydrodynamic effects to explain the differences between the patterns in Fig. 3.7. Nevertheless, the difference between the patterns observed for $c_{\mathrm{f}}=0$ and $c_{\mathrm{f}}=0.01 \mathrm{wt} \%$ is strong evidence that particle destabilisation plays a critical role for the pattern morphology, since the difference in surface tension and viscosity between these $c_{\mathrm{f}}$ is small, yet the contact line behaves completely differently.

Another feature of the thin saline water film is its thickness $h_{0}$, which governs the flow inside the film. Figure 3.8 shows a series of experiments in which we varied $h_{0}$ with $c_{\mathrm{d}}$ and $c_{\mathrm{f}}$ fixed, at $10 \mathrm{wt} \%$ and $30 \mathrm{wt} \%$, respectively. The ring-shaped pattern appears for all $h_{0}$, and the width of the ring increases

(a) $3 \mu \mathrm{m} \quad$ (b) $5 \mu \mathrm{m}$

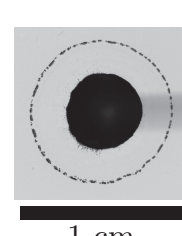

$1 \mathrm{~cm}$ (c) $10 \mu \mathrm{m}$

(d) $13 \mu \mathrm{m}$
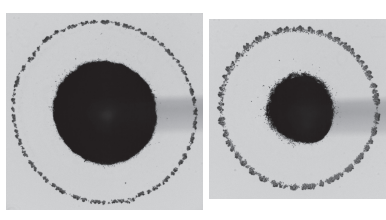

(e) $21 \mu \mathrm{m}$

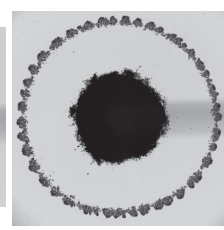

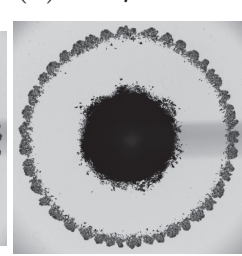

(f) $24 \mu \mathrm{m}$

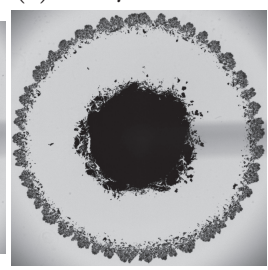

Figure 3.8: Patterns observed for (a) $h_{0}=3 \mu \mathrm{m}$, (b) $h_{0}=5 \mu \mathrm{m}$, (c) $h_{0}=10 \mu \mathrm{m}$, (d) $h_{0}=13 \mu \mathrm{m}$, (e) $h_{0}=21 \mu \mathrm{m}$, and (f) $h_{0}=24 \mu \mathrm{m}$. For all cases $t=1.00 \mathrm{~s}, c_{\mathrm{d}}=10 \mathrm{wt} \%$, and $c_{\mathrm{f}}=30 \mathrm{wt} \%$. The scale bar applies to all images. The width of the ring shows a strong dependence on $h_{0}$. 
with $h_{0}$. The radius of the ring also shows a small dependence on $h_{0}$, though small variations in the initial drop volume also affect the radius of the ring. The dependence of these features on $h_{0}$ indicate that hydrodynamic flow in the film plays an important role for the morphology of the ring-shaped pattern.

\subsection{Dynamical aspects of pattern formation}

The results presented in section 3.3 reveal that pattern formation is governed by delicate balance between hydrodynamic flow and particle destabilisation. Based on Figs. 3.4 and 3.5, we identify a separation of time scales - the initial spreading dynamics of the drop are $\mathcal{O}\left(10^{-2} \mathrm{~s}\right)$, whereas the outward motion of the ring is $\mathcal{O}(1 \mathrm{~s})$. In this section, we therefore study the dynamics of each of these two phases individually, to determine the effect of particle destabilisation on the flow dynamics.

\subsubsection{Rapid drop spreading}

When the drop first makes contact with the thin saline water film it spreads rapidly until it reaches an equilibrium shape (determined by surface energies). An example measurement of a spreading colloidal drop on a liquid substrate is shown in Fig. 3.9a. For low-viscosity liquids the spreading dynamics of a drop are typically explained by a balance between the capillary pressure $\left(\propto \gamma R / r_{\mathrm{b}}^{2}\right)$ and the inertial pressure $\left(\propto \rho\left(\mathrm{d} r_{\mathrm{b}} / \mathrm{d} t\right)^{2}\right)$, such that

$$
\frac{r_{\mathrm{b}}}{R} \propto\left(\frac{t}{\tau}\right)^{1 / 2}, \quad \text { with } \quad \tau=\sqrt{\frac{\rho R^{3}}{\gamma}},
$$

where $r_{\mathrm{b}}$ is the contact radius of the spreading drop (see Fig. 3.9a), $R$ is the initial radius of the drop before contact, $t$ is the time, $\tau$ is the inertiocapillary time scale, $\rho$ is the density of the drop, and $\gamma$ is the surface tension of the drop [103]. Importantly, Eq. 3.1 was found to be independent of the substrate properties [104]. Indeed, it was found that Eq. 3.1 is also valid for drop spreading on liquid substrates [84]. Since the surface tension of the thin saline water film is higher than that of the drop, the drop is not engulfed by liquid from the saline film, and thus the surface tension $\gamma$ in Eq. 3.1 is that of the drop $[84,86,87]$.

Figure $3.9 \mathrm{~b}$ shows the contact radius as a function of time, scaled according to Eq. 3.1, for several combinations of drop and film properties. The solid black line in Fig. 3.9b is Eq. 3.1 with fitted pre-factor 1.46, which is found to 


\subsection{Dynamical aspects of pattern formation}

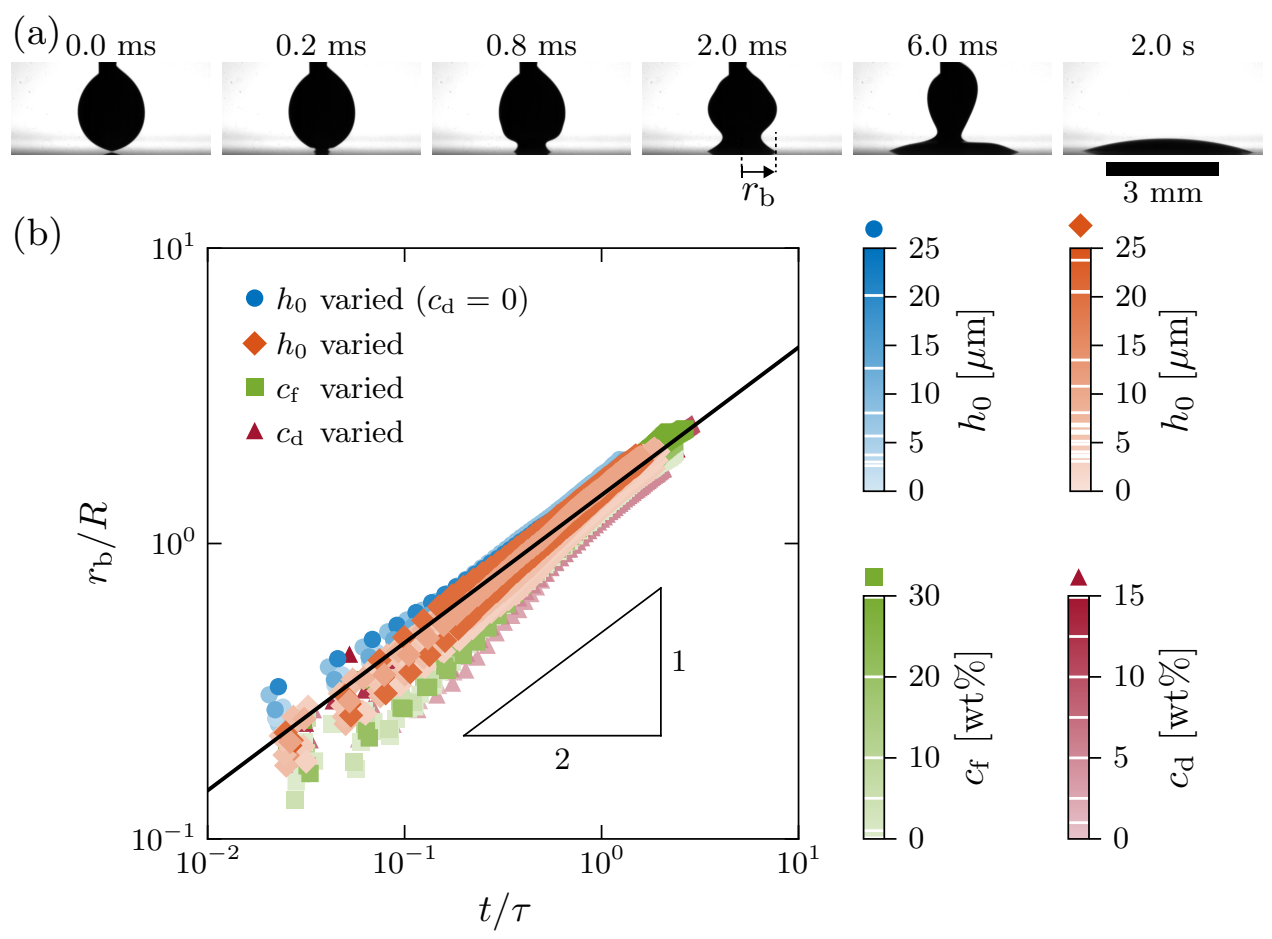

Figure 3.9: (a) A typical side view experiment showing the initial rapid spreading (here $c_{\mathrm{d}}=10 \mathrm{wt} \%, c_{\mathrm{f}}=30 \mathrm{wt} \%$, and $h_{0}=3 \mu \mathrm{m}$ ). (b) Contact radius $\left(r_{\mathrm{b}}\right.$, the definition is shown in the snapshot for $2.0 \mathrm{~ms}$ in (a)) versus time $(t)$. For all experiments, the reference system was $c_{\mathrm{d}}=10 \mathrm{wt} \%$, $c_{\mathrm{f}}=30 \mathrm{wt} \%$, and $h_{0} \approx 10 \mu \mathrm{m}$, with one of the parameters being varied in each of the experiment series (their values are indicated by the white lines in the colour bars, 45 experiments are shown in total). The solid black line shows Eq. 3.1 with pre-factor 1.46.

accurately describe the experimental data. A similar pre-factor was found for drop spreading on liquid substrates without colloidal particles in the drop [84]. Surprisingly, we find that the presence of colloidal particles in the drop and their destabilisation by the cations in the liquid substrate have no effect on the spreading dynamics - not even in the pre-factor of Eq. 3.1. We note that destabilisation of the particles occurs on the same time scale as drop spreading: undulation of the contact line is visible at the $t / \tau$-values covered in Fig. 3.9b. This indicates that particle destabilisation is limited to a small region close to 
the substrate surface, and that most of the colloidal particles in the bulk of the drop have not yet clustered when the drop is spreading. This is to be expected since diffusion governs the motion of cations into the pigment suspension [34]. The diffusion length scale associated with the time scale of inertial spreading is $L^{2} \approx D t$ [69]. For typical values associated with diffusion and inertial spreading $\left(D=\mathcal{O}\left(10^{-9} \mathrm{~m}^{2} / \mathrm{s}\right)\right.$, and $t=\mathcal{O}\left(10^{-2} \mathrm{~s}\right)$, see Fig. 3.4), we find $L=\mathcal{O}\left(10^{-6} \mathrm{~m}\right)$, which is orders of magnitude smaller than the drop size. Additionally, we expect that there is no significant flow-induced mixing of the two liquids, since the Reynolds number Re $=\rho\left(\mathrm{d} r_{\mathrm{b}} / \mathrm{d} t\right) h_{0} / \eta=\mathcal{O}(1)$. Clustering of particles is therefore limited to the regions of direct contact between the colloidal suspension and the thin saline water film.

\subsubsection{Slow translation of clusters}

The second phase of the temporal evolution of the pattern involves a slow radially outwards translation of the clustered particles in the ring. In Fig. 3.5 we showed that a ring forms regardless of the presence of particles, and that (for $c_{\mathrm{d}}=0$ ) a hole opens in the film at the centre. This is reminiscent of "Marangoni spreading"-flow induced in the thin films by a surface tension gradient [105]. We recall that the surface tension of the saline water film is always higher than that of the drop in our experiments (Fig. 3.2). In Marangoni spreading, the liquid of the substrate that is displaced by the flow is collected in a "rim" (c.f., Fig. 3.5 and Ref. [105]). We hypothesise that the particle clusters in the ring are transported at the crest of this rim. Particles have been shown to be transported by Marangoni waves [106]. If the particle ring is indeed transported by the Marangoni rim, then the radial growth dynamics of the cluster ring should be equal to the growth dynamics of Marangoni spreading.

Following the method of Ref. [105] we can derive a scaling law for the expected time evolution of the radius of the ring $r_{\mathrm{r}}$. The flow inside the liquid substrate is driven by the Marangoni stress $\Delta \gamma / r_{\mathrm{r}}$ and opposed by the viscous stress $\eta\left(\mathrm{d} r_{\mathrm{r}} / \mathrm{d} t\right) / h$. Here, $h$ is the characteristic thickness of the thin liquid film that remains on the substrate for radii $<r_{\mathrm{r}}$ (see the schematic in Fig. 3.10), which is determined from volume conservation: the liquid volume in the thin centre region is equal to the initial drop volume, such that $\pi r_{\mathrm{r}}^{2} h \sim V$. By balancing the stresses, we then find

$$
r_{\mathrm{r}} \propto K t^{1 / 4}, \quad \text { with } \quad K=\left(\frac{V \Delta \gamma}{\eta}\right)^{1 / 4}
$$




\subsection{Dynamical aspects of pattern formation}
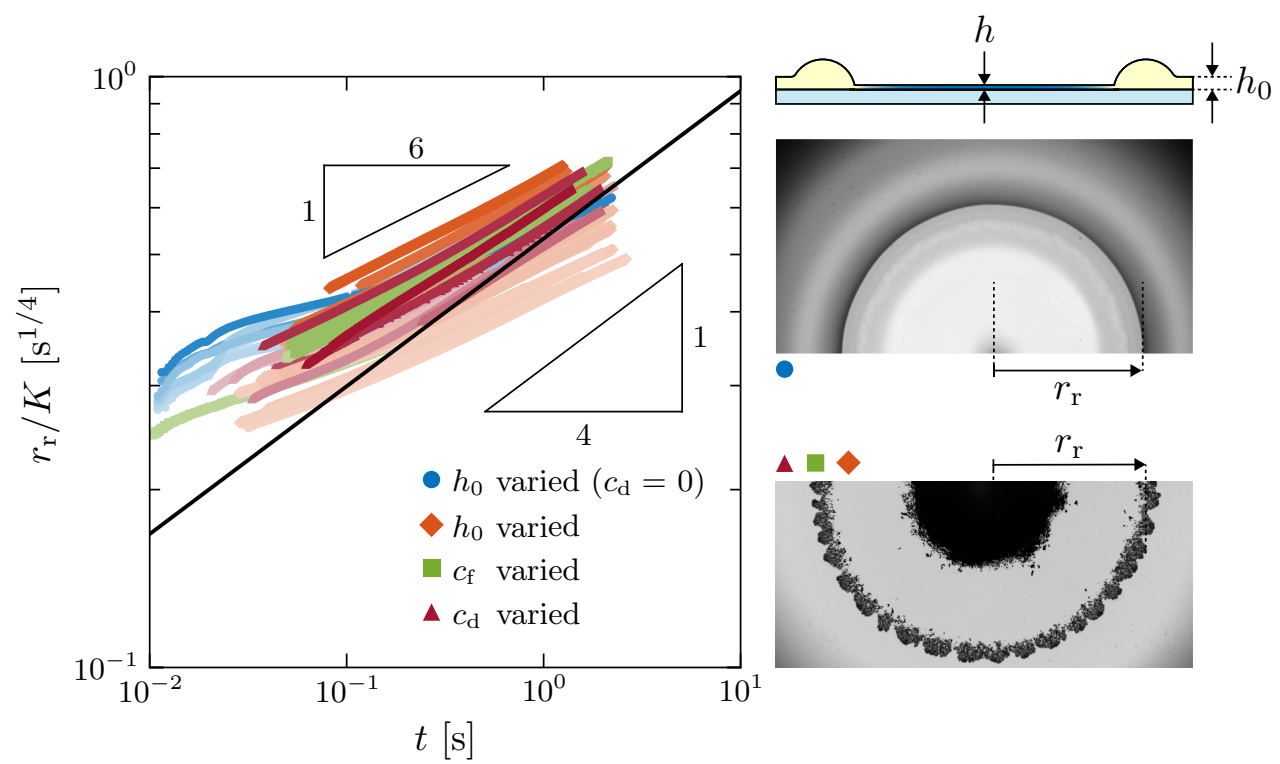

Figure 3.10: Ring radius $\left(r_{\mathrm{r}}\right)$ versus time $(t)$. For all experiments, the reference system was $c_{\mathrm{d}}=10 \mathrm{wt} \%, c_{\mathrm{f}}=30 \mathrm{wt} \%$, and $h_{0} \approx 10 \mu \mathrm{m}$, with one of the parameters being varied in each of the experiment series. The solid black line shows Eq. 3.2 with pre-factor 0.54. The colour bar legend from Fig. 3.9 also applies to this figure. The schematic shows the definitions of $h$ and $h_{0}$ used in the derivation of Eq. 3.2. The snapshots show the definitions of $r_{\mathrm{r}}$ for $c_{\mathrm{d}}=0$ (top) and $c_{\mathrm{d}}>0$ (bottom).

where $r_{\mathrm{r}}$ is the radius of the ring, $V$ is the drop volume, and $\eta$ is the viscosity of the liquid substrate.

In Fig. 3.10 we show the ring radius as a function of time, scaled according to Eq. 3.2, for several combinations of drop and film properties. The images show the definitions of $r_{\mathrm{r}}$. For $c_{\mathrm{d}}>0$, where the pattern is visible, we measure $r_{\mathrm{r}}$ by fitting a circle to the ring. For $c_{\mathrm{d}}=0$ this method cannot be used, and we measure $r_{\mathrm{r}}$ by fitting a circle to the inner rim, i.e., the location where the image intensity gradient is maximum. The solid black line in Fig. 3.10b is Eq. 3.2 with fitted pre-factor 0.54. While Eq. 3.2 correctly predicts the order of magnitude of $r_{\mathrm{r}}$, the experimental data does not precisely match the predicted exponent $1 / 4$. Rather, we find that an exponent of approximately $1 / 6$ best matches the data. A possible reason for this is that Eq. 3.2 does 

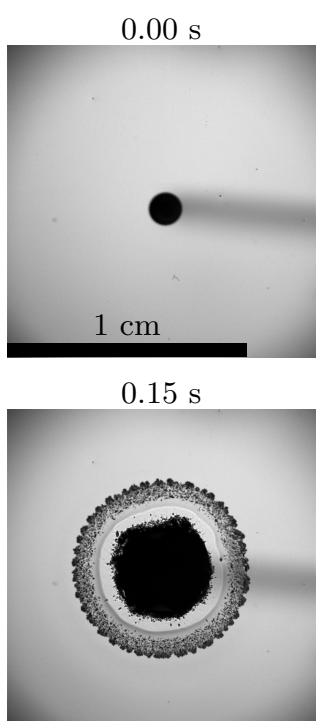

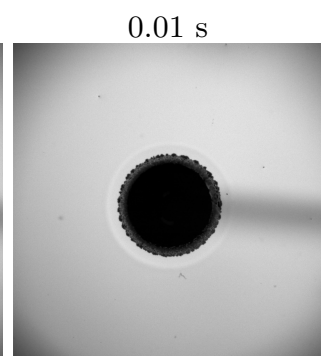

$0.50 \mathrm{~s}$

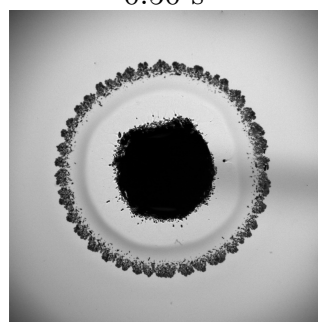

$0.02 \mathrm{~s}$

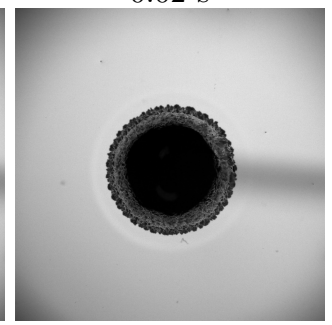

$1.00 \mathrm{~s}$

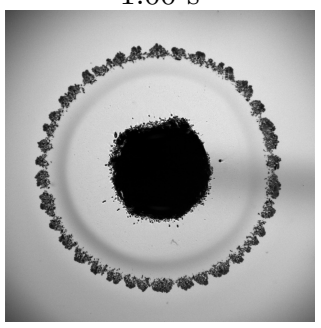

$0.04 \mathrm{~s}$

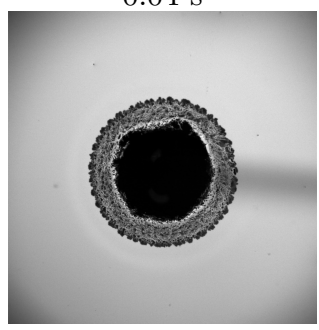

$2.00 \mathrm{~s}$

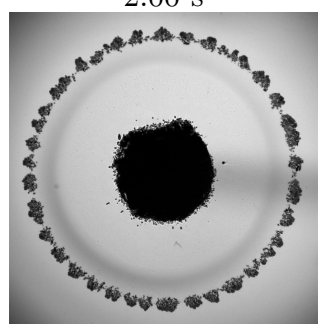

Figure 3.11: Image sequence of the deposition of a $10 \mathrm{wt} \%$ colloidal drop on a $30 \mathrm{wt} \% \mathrm{CaCl}_{2}$ solution film $\left(h_{0}=15 \mu \mathrm{m}\right)$, and the subsequent pattern formation. The clustered particles in the ring are located on the crest of the Marangoni rim. We used the same illumination technique as in Fig. 3.5.

not include the finite size of the centre drop - the drop eventually reaches an equilibrium shape with a contact angle larger than zero (see for example Fig. $3.9 \mathrm{a}$ at $t=2.0 \mathrm{~s}$ ). Additionally, the initial inertial spreading dynamics and subsequent crossover into the Marangoni spreading regime (described in section 3.4.1) are also not included in Eq. 3.2. This crossover can be observed for the $c_{\mathrm{d}}=0$ data in Fig. 3.10 close to $t \approx 10^{-2} \mathrm{~s}$, and is associated with a slowing down of the radial growth. However, we note that the data for various $c_{\mathrm{f}}$ (with $3.5 \mathrm{mN} / \mathrm{m} \leq \Delta \gamma \leq 11.1 \mathrm{mN} / \mathrm{m}$ ) collapse with the scaling predicted by Eq. 3.2. Further evidence that the particle clusters are surfing on the Marangoni rim is provided by the overlap of the ring radius for $c_{\mathrm{d}}>0$ with that of the $c_{\mathrm{d}}=0$ data.

Finally, in Fig. 3.11 we show the pattern formation dynamics for $c_{\mathrm{d}}>0$ with diverging back illumination technique that was also used for $c_{\mathrm{d}}=0$ in Fig. 3.5. This measurement confirms that the particle clusters are transported by the crest of the Marangoni rim. We do note that the presence of particles seems to have a smoothing effect on the transition from the Marangoni rim to 


\subsection{Cluster shape and proposed mechanism}

the thin central region (c.f., Fig. 3.5, where a sharp transition can be observed). Hence, the presence of particle clusters does seem to affect the morphology of thin film's interface in the Marangoni spreading regime.

\subsection{Cluster shape and proposed mechanism}

Having identified the general qualitative features (section 3.3) and dynamics (section 3.4) of the patterns, we now propose a mechanism that could lead to the observed ring-shaped patterns. In particular, we provide an explanation for the final shape of the individual clusters that constitute the ring.

The first spreading phase (section 3.4.1) is rapid, such that the destabilisation of the colloidal particles by $\mathrm{Ca}^{2+}$ cations occurs mostly at areas of direct contact between the drop and the film. At the contact line, the destabilised particle clusters form an undulate shape, see Fig. 3.12a. These undulations play a large role for the final pattern morphology. The spreading dynamics slow down after the initial (inertial) spreading phase, and the radial growth is then driven by Marangoni spreading (section 3.4.2). During the crossover between the two spreading regimes, particle clusters separate from the centre drop (see Fig. 3.12b), carried by the Marangoni rim (Fig. 3.11). This indicates that liquid from the colloidal drop (or small particle clusters) is transported onto the rim by Marangoni flow before the rim separates from the centre drop (we recall that the surface tension of the colloidal drop is lower than that of the saline water film). The small particle clusters that are trapped on the Marangoni rim eventually aggregate to form the large clusters that constitute the ring. This aggregation is illustrated in Fig. 3.12c - the radial velocity of small clusters (the small circles indicated by (i) in Fig. 3.12c) close to the centre drop is higher than the radial velocity of particles close to (or at) advancing front, due to curvature-driven motion or capillary interactions between particles $[70,107]$. Compare, for example, the width of the ring in Fig. 3.12b-d.

As the radius (and thus also the circumference) of the Marangoni rim on which the ring is trapped keeps growing, Van der Waals forces are no longer able to keep the clustered particles at the advancing front together. This results in v-shaped cracks in the advancing front of the ring (Fig. 3.12c), and, eventually, break-up of the ring into a number of large clusters (Fig. 3.12d). The location of the cracks is determined by the initial undulation of the contact line. Compare, for example, the location of the cracks in Fig. 3.12c to the maximum radial extent of the undulations in Fig. 3.12a (the white dashed lines 

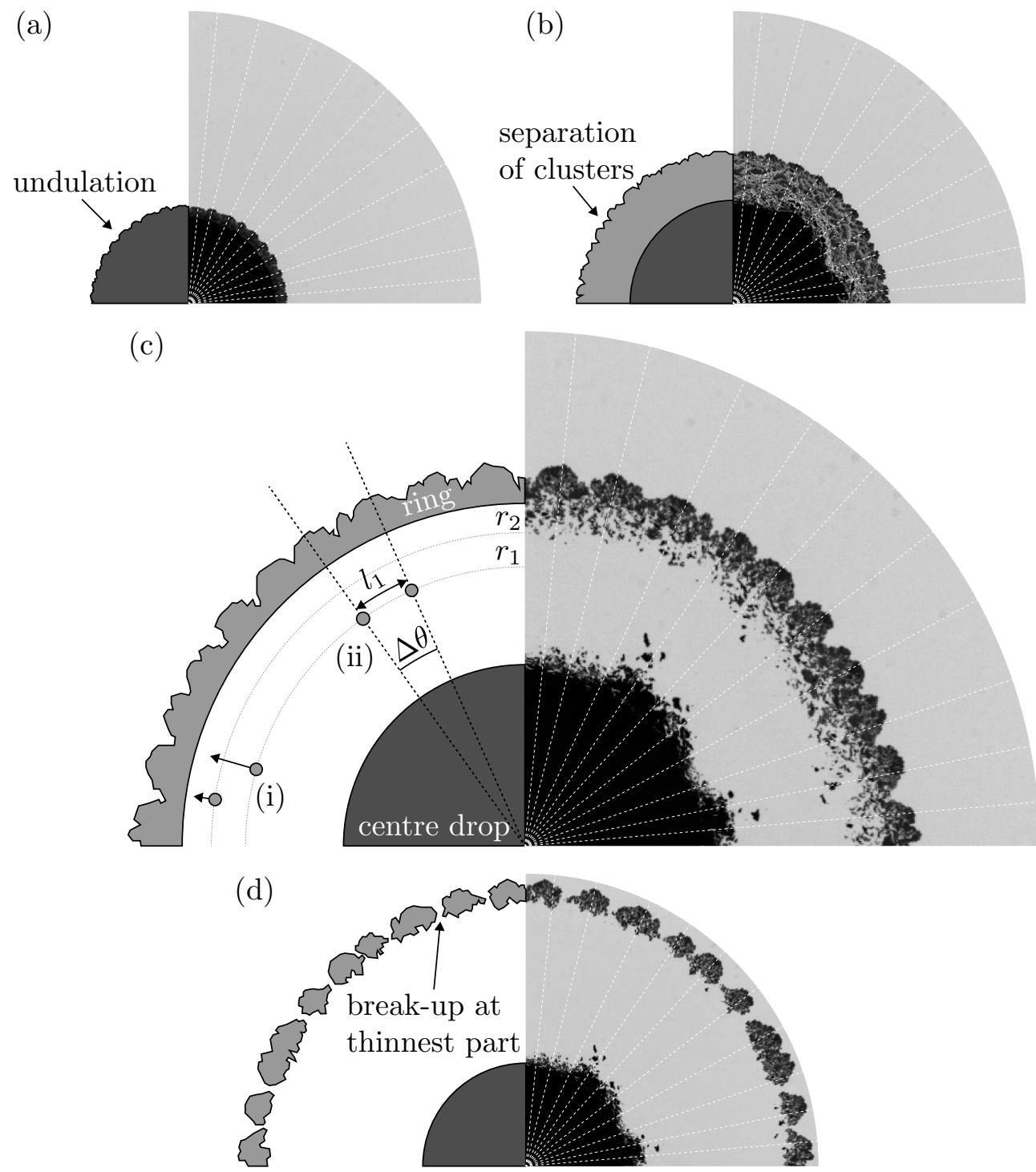

Figure 3.12: Overview of the different phases of the pattern formation mechanism. (a) The contact line develops an undulating shape during the initial inertial spreading phase. (b) Small particle clusters (light grey region) separate from centre drop (dark grey region). Particles at radius $r_{1}$ have a higher velocity than particles are radius $r_{1}$. The undulating shape of the outer edge is maintained (the white dashed lines serve as guides to the eye). (c) The separated clusters collect in a ring. (d) The ring breaks up into separate large clusters. Break-up occurs at the thinnest parts of the ring. 


\subsection{Cluster shape and proposed mechanism}

serve as guides to the eye). Cracks appear at places where the ring is thinnest (i.e., the weakest parts of the ring), and these align with the undulation minima. At the rear of the ring, however, there is a continuous addition of small clusters to the ring, through the process described above. Since these clusters move radially outward with no perpendicular motion, they are distributed over an increasingly large circumference. This is illustrated in Fig. 3.12c - two particles, indicated by (ii), a distance $r_{1}$ from the centre that are separated by an angle $\Delta \theta$ will be separated by a distance $l_{1}=r_{1} \Delta \theta$. Some time later, when the particles are at a distance $r_{2}$ from the centre (with $r_{2}>r_{1}$ ), their distance is $l_{2}=r_{2} \Delta \theta>l_{1}$. Hence, while the ring cracks at the advancing front, the particles are evenly distributed at the rear of the ring (though the particle density decreases with increasing radius). This leads to the distinct trapezoidal shape of the particles, observed in Fig. 3.12d.

This mechanism also offers an explanation for the width $w$ of the ring,

(a)

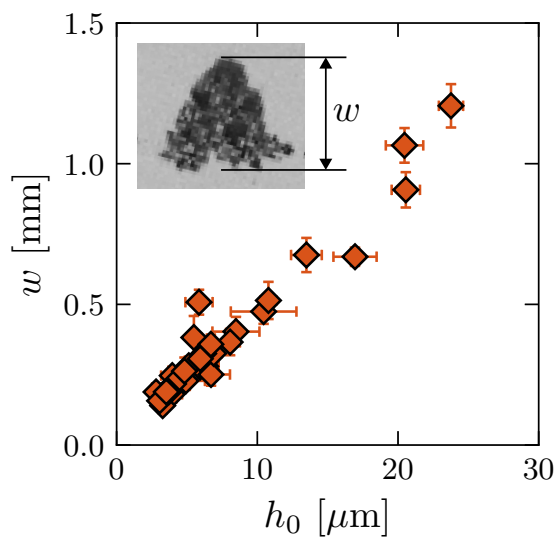

(b)

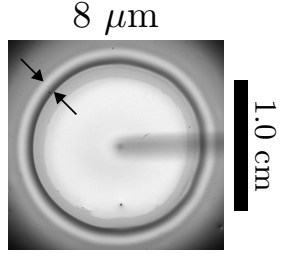

(c)

$20 \mu \mathrm{m}$

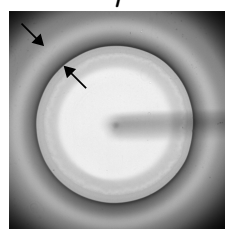

Figure 3.13: (a) Average width of the particle clusters on the Marangoni rim $(w)$ as a function of film thickness $\left(h_{0}\right)$ for $c_{\mathrm{d}}=10 \mathrm{wt} \%$. The inset show the definition of $w$, which we measure for each individual particle cluster; the reported value is an average of all these values, with the error bars indicating the standard deviation. Note that the width of the Marangoni rim also increases with $h_{0}$, as indicated by the snapshots in (b) and (c), where $c_{\mathrm{d}}=0$ (the rim width is indicated by the arrows). For all cases $c_{\mathrm{f}}=30 \mathrm{wt} \%$ was fixed. 
which increases with $h_{0}$ (see Fig. 3.9). The total number of particles on the Marangoni rim at the time of separation from the centre drop is set by the width of the rim, which has been shown to depend on $h_{0}$ (in the absence of particles) [105]. Hence, the final width of the ring $w$, which is determined by the number of particles on the rim, is expected to scale with $h_{0}$ as well. This is verified in Fig. 3.13a, where we report $w$ as a function of $h_{0}$. Figure $3.13 \mathrm{~b}-\mathrm{c}$ show two snapshots of the Marangoni rim for different $h_{0}$, and show that the width of the Marangoni rim (in the absence of particles) indeed depends on $h_{0}$, in accordance with Ref. [105].

Finally, we remark that the precise origin of the undulate shape of the contact line during the initial spreading phase remains unknown. We note, however, that cracking of the contact line (similar to the mechanism described above) could be a possible source of the undulation. Here, the contact line forms a continuous chain of clustered particles. Since the radius of the drop increases due to its inertial spreading, it is forced to break up. Subsequently, new particles clusters join behind the (broken-up) contact line, giving rise to its undulate shape.

\subsection{Conclusion and outlook}

In this chapter, we have revealed a novel pattern formation mechanism: ringshaped patterns appear when a colloidal suspension drop is deposited on a thin saline water film. Using experiments, we have shown that the patterns appear due to a simultaneous hydrodynamic flow and particle destabilisation. The morphological features of the patterns depend on a number of parametersthe particle concentration and salt concentration need to be sufficiently high for the ring-shaped patterns to appear, and the width of the ring is strongly affected by the thickness of the liquid substrate.

The contact line plays an important role in the formation of the pattern. The contact line dynamics are initially well-described by inertial flow, until the drop reaches its final radius. Then, clustered particles separate from the drop and are transported at the crest of the Marangoni rim, resulting in the final ring-shaped pattern.

We note that the exact moment of separation of the particles from the centre drop is not yet well-understood - it is not yet clear if liquid from the saline water film remains underneath the centre drop after the ring with particle clusters separates, or how the presence of the particles affects the (threedimensional) morphology of the contact line region. Our current experimental 


\subsection{Conclusion and outlook}

method is not suited to study the flow dynamics in the liquid substrate directly underneath the centre drop due to the opaque nature of the particle suspension. Other techniques, such as digital holographic microscopy, confocal microscopy or even numerical simulations, may be more suited for such explorations [81].

The results presented in this chapter show that physico-chemical hydrodynamics contains rich physics. In terms of applications, our results are particularly relevant for inkjet printing technology, where salt-containing primer layers are often used to improve print quality. In chapter 2, we showed that the presence of a primer layer can introduce unwanted interactions between ink drops. Here, we show that the physico-chemical nature of ink and the primer liquid can also induce unwanted effects that must be accounted for in their design. We do note that the ratio between drop size $R$ and film thickness $h_{0}$ in our work is much larger than in inkjet printing, and it would therefore be of interest to study patterns at smaller $R / h_{0}$-ratios.

Future work should address the effect that the material properties of the salt and the particles have on the observed patterns. For example, it has been shown that using $\mathrm{MgCl}_{2}$ instead of $\mathrm{CaCl}_{2}$ results in denser particle clusters, which could in turn affect the pattern [34]. Furthermore, the destabilisation of colloidal particles is also affected by the valency of the cations [108]. A more detailed understanding of these properties could also provide better insight into the appearance of the contact line undulation, which is expected to depend on the (destabilisation) properties of the particles. A better understanding of the effect of these material properties, and others such as the particle size and shape, will also allow tailoring of the salt and particles to the desired outcome: no appearance of the pattern, which is desired in inkjet printing, or the contrary, which may have applications in micro-patterning. 


\subsection{Appendix}

\subsubsection{Evaporation of the thin films}

Figure 3.14 shows a typical example of a temporal thickness measurement of a saline water film $\left(c_{\mathrm{f}}=30 \mathrm{wt} \%\right)$. The film was immediately transferred to the spectrometer setup upon completion of its preparation by spin-coating. Here, $t=0$ corresponds to the start of the reflectometry measurement. The measurement was performed in ambient conditions, i.e., $T=20 \pm 1^{\circ} \mathrm{C}$, $R H=64 \pm 3 \%$ (measured with a Super EX Sensor, TFA).

We observe that the film thickness changes only slightly over the course of several minutes, despite the volatile nature of water. We suspect that this is due to the formation of a thin salt crust that blocks water molecules. Water evaporates from the surface of a fresh film (i.e., without crust). This causes a local increase of the $\mathrm{CaCl}_{2}$ concentration close to the surface, which eventually results in the formation of a solid crust at the surface of the film when the local $\mathrm{CaCl}_{2}$ concentration exceeds the solubility limit. A similar crust-forming effect due to evaporation has been reported for spin-coated polymer films [109]. When a water drop is deposited on a thin saline water film that has been exposed to ambient conditions, we observe radial lines that could be the result of wrinkling of the salt crust, see Fig. 3.15. These patterns are very similar to the wrinkling patterns that appear when an object is dropped on a thin elastic

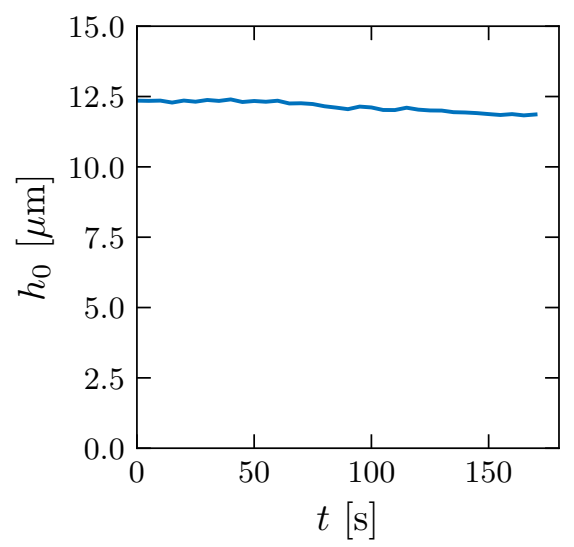

Figure 3.14: Thickness $\left(h_{0}\right)$ as a function of time $(t)$ for a thin saline water film $\left(c_{\mathrm{f}}=30 \mathrm{wt} \%\right)$ exposed to ambient conditions $\left(T=20 \pm 1^{\circ} \mathrm{C}\right.$, $R H=64 \pm 3 \%)$. 


\subsection{Appendix}

sheet floating on a deep liquid bath, c.f., Ref. [110]. Note that the wrinkling pattern in Fig. 3.15 becomes less prominent with increasing film thickness.

The experiments described in the main text (including the film thickness measurements) were completed within two minutes after completion of the spin-coating procedure, such that evaporation of the film does not play a large role in the experiments.

(a) $4 \mu \mathrm{m}$

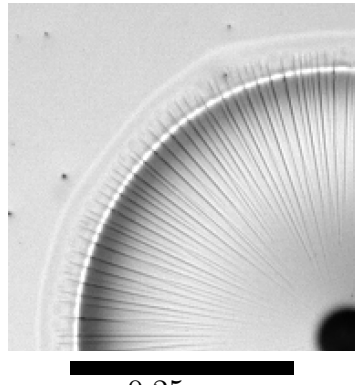

$0.25 \mathrm{~cm}$ (b) $11 \mu \mathrm{m}$

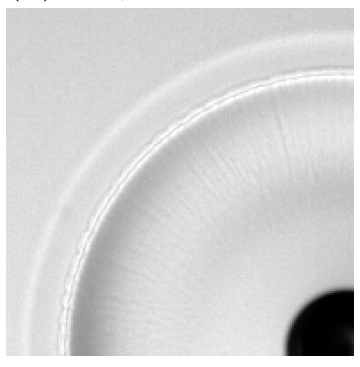

(c) $23 \mu \mathrm{m}$

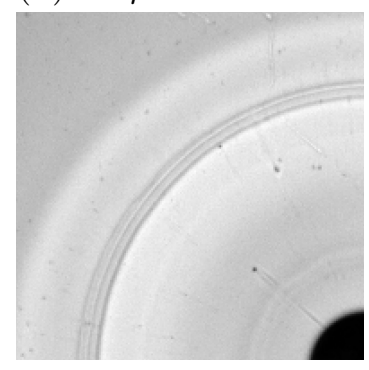

Figure 3.15: Wrinkling-like pattern that is observed (at $t \approx 10 \mathrm{~ms}$ ) when a water drop is deposited on a thin saline water film $\left(c_{\mathrm{f}}=30 \mathrm{wt} \%\right)$ that has been exposed to ambient conditions, for (a) $h_{0}=4 \mu \mathrm{m}$, (b) $h_{0}=11 \mu \mathrm{m}$, (c) $h_{0}=23 \mu \mathrm{m}$. 



\section{Chapter 4}

\section{Wetting of two-component drops: Marangoni contraction versus autophobing}

The wetting properties of multi-component liquids are crucial to numerous industrial applications. The mechanisms that determine the contact angles for such liquids remain poorly understood, with many intricacies arising due to complex physical phenomena, for example, due to the presence of surfactants. In this chapter, we consider two-component drops that consist of mixtures of vicinal alkanediols and water. These diols behave surfactant-like in water. However, the contact angles of such mixtures on solid substrates are surprisingly large. We experimentally reveal that the contact angle is determined by two separate mechanisms of completely different nature, namely Marangoni contraction (hydrodynamic) and autophobing (molecular). The competition between these effects can even inhibit Marangoni contraction, highlighting the importance of molecular structures in physico-chemical hydrodynamics.

(1) Published as: Michiel A. Hack ${ }^{\bullet}$, Wojciech Kwieciński ${ }^{\bullet}$, Olinka Ramírez-Soto ${ }^{\bullet}$, Tim Segers, Stefan Karpitschka, E. Stefan Kooij, and Jacco H. Snoeijer, Wetting of TwoComponent Drops: Marangoni Contraction Versus Autophobing, Langmuir 37, 3605-3611 (2021). • Contributed equally. M. A. H. performed the contact angle and surface tension measurements, W. K. performed the ellipsometry measurements, O. R.-S. performed the $\mu \mathrm{PIV}$ measurements. 


\subsection{Introduction}

Many industrial processes require a fundamental understanding of the wetting properties of liquids on solid surfaces [111]. Examples are inkjet printing [7], oil recovery [112], and lithography [6]. A key concept in the description of wetting is the contact angle $\theta$, as defined in Fig. 4.1a. Properties of the liquid together with the surface chemistry of the solid determine the value of $\theta[16,113]$. The wetting properties and contact angles of single-component liquids have been extensively studied $[69,114]$. However, a large number of industrial applications require mixtures of liquids [115] or the addition of a surfactant to enhance the spreading properties of a liquid [116]. For complex drops consisting of two or more components, the wetting properties are far from understood. The components may phase separate $[24,117]$, selectively evaporate [118], emulsify [25], and adsorb at interfaces [29], and even gravity can play a role $[119,120]$, leading to intricate wetting properties on solid surfaces.

In this chapter, we study the contact angle $\theta$ of multi-component drops, where the less volatile component acts as a surfactant, on OH-terminated substrates that are fully wetted by water. Figure $4.1 \mathrm{a}$ shows the contact angle of drops consisting of water-1,2-hexanediol (1,2-HD) mixtures on a piranha solution-cleaned hydrophilic glass substrate (microscope coverslips, MenzelGläser) with minimal pinning. The reported angle is attained within seconds after deposition of the drop, see Fig. 4.1b. The key result of Fig. 4.1a is that $\theta$ continually increases with the 1,2-HD mass fraction $\phi$. This is surprising for two reasons. First, 1,2-HD has been shown to exhibit surfactant-like properties when mixed with water due to its amphiphilic molecular structure [121-124]. Increasing the mass fraction $\phi$ of 1,2 -HD lowers the surface tension $\gamma_{\mathrm{LV}}$, see Fig. $4.1 \mathrm{c}$, which normally would lead to enhanced spreading. However, the opposite trend is found: $\theta$ increases with $\phi$. A second surprise is that this increase continues above the critical micelle concentration $(\mathrm{cmc}) \phi_{\mathrm{cmc}} \approx 0.1$, even though $\gamma_{\mathrm{LV}}$ is constant in this range [125]. Here we show that these unexpected features are the result of two mechanisms of different origins-one of hydrodynamic nature: Marangoni contraction, and the other of molecular nature: autophobing. This resolves the relation between two controversial models for Marangoni contraction [19, 126, 127] and, for the first time, describes quantitative limitations of the contracted state and its sensitivity to the molecular structure of the surface active component. 


\subsection{Introduction}
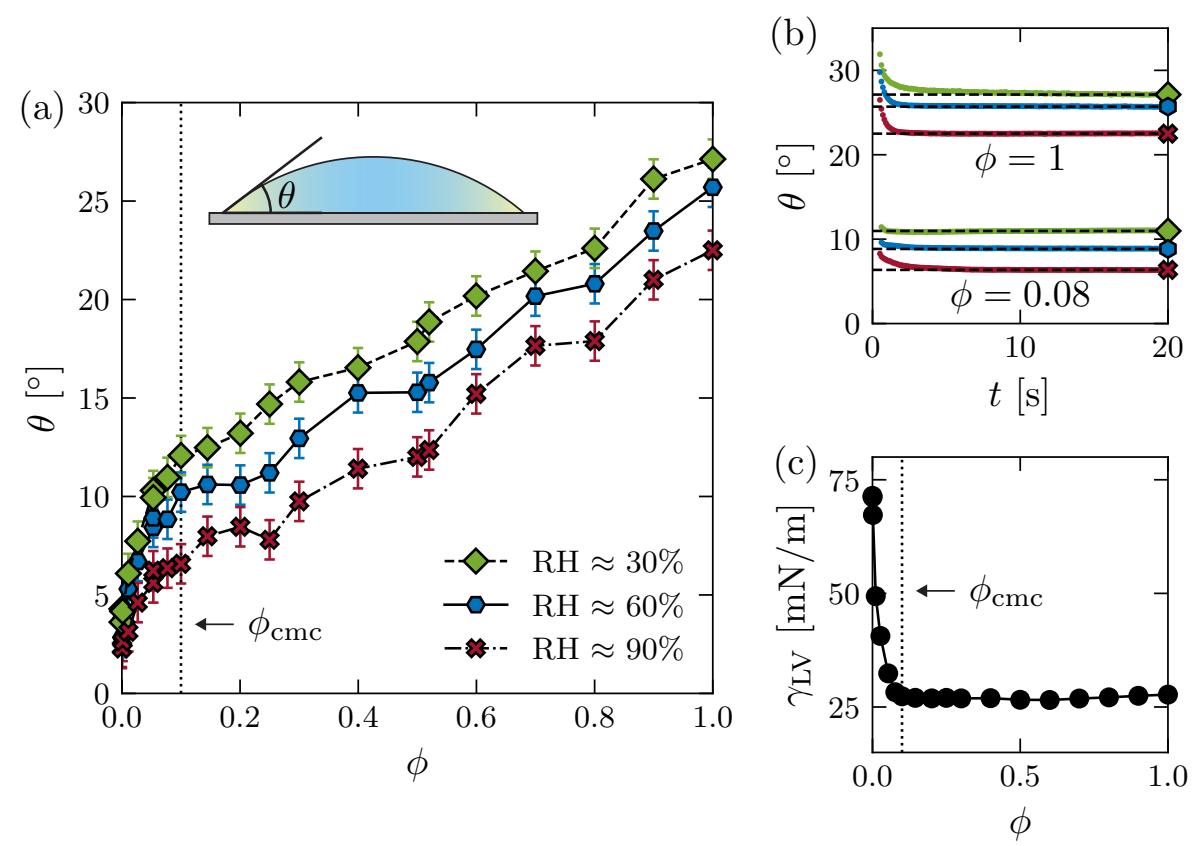

Figure 4.1: (a) Contact angle $(\theta)$ of water-1,2-HD mixtures as a function of the mass fraction $(\phi)$ of 1,2 -HD for various relative humidities $(\mathrm{RH})$. The vertical dotted line indicates the cmc $\left(\phi_{\mathrm{cmc}} \approx 0.1\right)$. Schematic: Definition of $\theta$. The mass fraction of 1,2-HD (yellow) is higher near the contact line due to selective evaporation. (b) Evolution of $\theta$ over time $(t)$ for $\phi=0.08$ (bottom three lines) and $\phi=1$ (top three lines) for various $\mathrm{RH}$. The drops are gently placed at $t=0$, after which a rapid decrease of $\theta$ to a quasi-steady value is observed. The horizontal black dashed lines and large markers (at $t=20 \mathrm{~s}$ ) indicate averages of all values in the quasi-steady regions (from $t \approx 10 \mathrm{~s}$ onwards) and correspond to the datapoints in (a). (c) Surface tension $\left(\gamma_{\mathrm{LV}}\right)$ of water-1,2-HD mixtures, measured using the pendant drop method. 


\subsection{Experimental methods}

\subsubsection{Contact angle measurements}

The contact angle $\theta$ was determined from the side-view images (obtained using a Ximea XiQ MQ013MG-ON camera with Zeiss Makro-Planar 1:2.8 f=60 mm lens with Olympus ILP-2 light source). We determined $\theta$ by fitting a circle to the drop interface and a straight line to the substrate. The height $H$ and base radius $R$ of the drop are extracted from the circle fit and used to calculate the contact angle using $\theta=2 \tan ^{-1}(H / R)$. The uncertainty in the contact angle, which originates from the pixel error and small variations in time, see Fig. $4.1 \mathrm{c}$, is estimated to be $\pm 1^{\circ}$. The $\mathrm{RH}$ was controlled using a home-built apparatus (for details see Ref. [53]), and was constantly monitored along with temperature $T$ during the measurement using a sensor (Honeywell HIH6130) in the setup. Example measurements of the time evolution of $\theta$ for $\phi=0.08$ and $\phi=1$ are shown in Fig. 4.1b.

\subsubsection{Surface tension measurements}

The surface tension measurements were performed using the pendant drop method [100]. For each aqueous solution of 1,2-HD, the surface tension of ten drops of $2.5 \mu \mathrm{L}$ was measured $\left(T=2{ }^{\circ} \mathrm{C}, \mathrm{RH}=45 \%\right)$, with ten images collected for each drop over a period of $1 \mathrm{~s}$. The surface tensions $\gamma_{\mathrm{LV}}$ reported in Fig. 4.1c are an average of these measurements (i.e., 100 images per datapoint), with an average error of $0.57 \mathrm{mN} / \mathrm{m}$.

\subsubsection{Micro-particle image velocimetry measurements}

The flow velocities within evaporating binary drops of 1,2-HD and water were quantified by micro-particle image velocimetry. We used fluorescent polystyrene microspheres (Thermo Fisher Scientific F8809, $0.2 \mu \mathrm{m}$ diameter, stock solution concentration $2 \% \mathrm{w} / \mathrm{v}$ ) as tracers, with a mass fraction of $7.8 \times 10^{-5}$ of the particle stock solution in the final mixture. The particles within the drops were visualised with an inverted epifluorescence microscope (Nikon Eclipse Ti2), equipped with a water immersion objective (Nikon CFI APO LWD $20 \times \mathrm{WI}$ ) with a numerical aperture of 0.95 . Thin correlation depths (i.e., high plane selectivity) require diffraction-limited imaging. To achieve this not only close to the substrate but also in the bulk fluid, the refractive index of the immersion medium has to be close to that of the working medium, for which water immersion objectives are ideally suited. The focal plane was parallel to 


\subsection{Experimental methods}
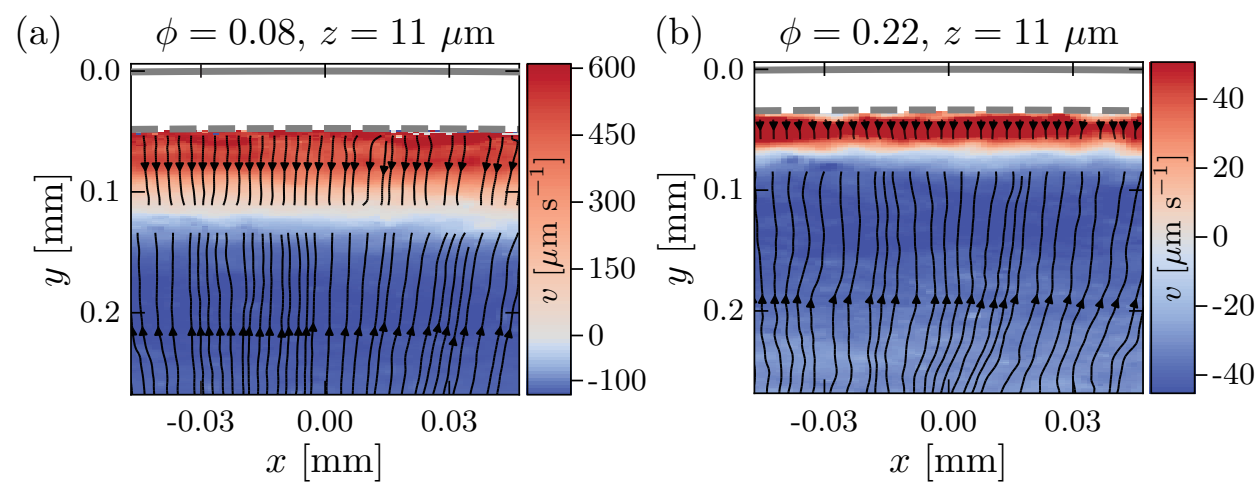

Figure 4.2: Example velocity fields in the $z$ plane for (a) $\phi=0.08$, $\mathrm{RH}=71 \%$ and (b) $\phi=0.22, \mathrm{RH}=40 \%$. The thick grey line denotes the three-phase contact line at the substrate $(z=0)$. The dashed line indicates the interface of the drop at $z=11 \mu \mathrm{m}$.

the substrate and moved in the vertical direction with the closed-loop focusing stage of the microscope. The time required to switch between planes was less than 100 ms. For each $z$ plane, a sequence of approximately 500 frames was recorded with a high speed camera (Phantom VEO 4K 990L, imaging speed at $900-1000 \mathrm{fps}$ ). Thus, the time required for a full $z$-scan was on the order of approximately $10 \mathrm{~s}$, much shorter than the time scale on which the flow velocities change for a quasi-stationary drop. This was checked by comparing data from successive upward and downward scans. To evaluate the flow velocities, the images were analysed with an in-house developed cross-correlation based algorithm with adaptive interrogation window sizes and correlation averaging over approximately 100 frames. The analysis was implemented through the Python API of Tensor Flow, to enable fast computation on graphics processing units. Example velocity fields in the $z$ plane are shown in Fig. 4.2. The velocities presented in Fig. 4.3 were obtained by azimuthally averaging over approximately $100 \mu \mathrm{m}$. Additionally, simultaneous shadowgraphy of the drop contour was performed to record the contact angle with a second camera (Point Grey Grasshopper2, imaging speed at $27 \mathrm{fps}$ ) through a macro lens (Thorlabs Bi-Telecentric lens, $1.0 \times$, working distance $62.2 \mathrm{~mm}$ ). Experiments were conducted in a humidity-controlled chamber mounted on top of the microscope. As substrates, we used piranha-cleaned microscope coverslips (Menzel Gläser). 

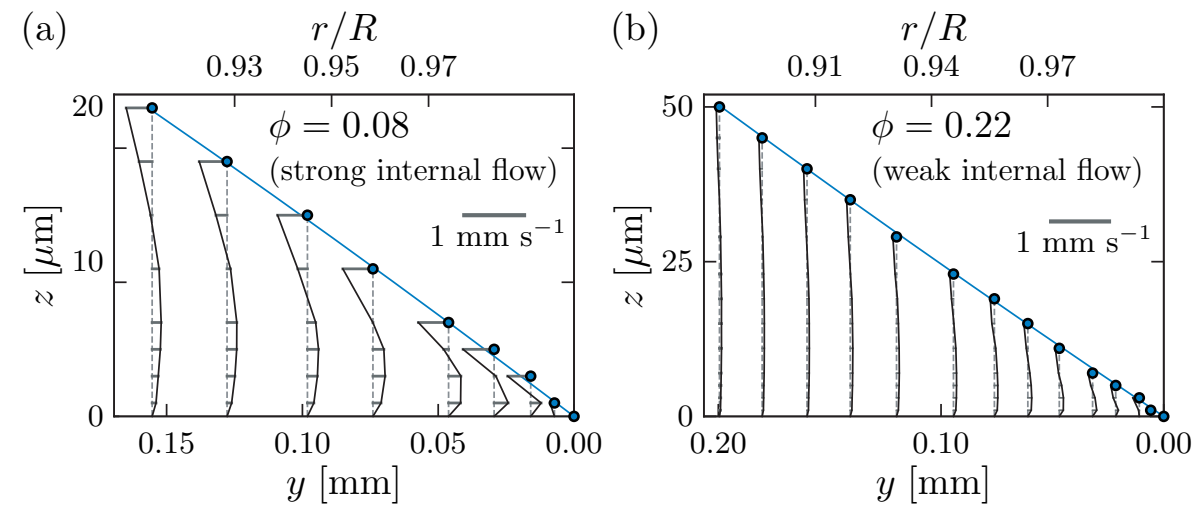

Figure 4.3: Horizontal velocity component in the drops measured using high resolution micro-particle image velocimetry. The blue line indicates the outer surface of the drop. The horizontal lines indicate the velocity, where the direction is indicated by the location with respect to the vertical dashed line. (a) Velocity field for $\phi=0.08$ and $\mathrm{RH}=71 \%\left(\theta=9^{\circ}\right)$. (b) Velocity field for $\phi=0.22$ and $\mathrm{RH}=40 \%\left(\theta=14^{\circ}\right)$, which is significantly weaker than that in (a).

\subsubsection{Ellipsometry measurements}

The ellipsometry measurements (J. A. Woolam Co. VB-400-VASE ellipsometer with WVASE32 software) were performed on $2 \times 2 \mathrm{~cm}^{2}$ piranha solutioncleaned silicon (100) substrates (Okmetic) in ambient conditions $\left(T=21{ }^{\circ} \mathrm{C}\right.$, $\mathrm{RH}=40 \pm 5 \%)$. The thickness $d$ of the layer of adsorbed molecules was obtained by fitting the obtained ellipsometric spectrum to a model of a surface composed of a silicon substrate with a native oxide layer and the Cauchy layer on top. The thickness of the native oxide layer (typically $1.8 \mathrm{~nm}$ for these substrates) was determined for each substrate separately before performing the adsorption experiments. The Cauchy layer is an empirical model for the dependence of the refractive index on the wavelength of a dielectric layer:

$$
n(\lambda)=A+B / \lambda^{2}+C / \lambda^{4}+\ldots,
$$

where $n$ is the refractive index, $\lambda$ is the wavelength of the light that is used, and $A, B$, and $C$ are the material-dependent empirical coefficients [128]. Here, we used the values $A=1.45, B=0.1$, and $C=0$, and all other higher order terms were set to zero. 


\subsection{Marangoni contraction}

During the measurement, the substrate is vertically placed above a Teflon container. A sketch of this configuration is available in the appendix. A dynamic scan $\left(3.5 \mathrm{eV}, 75^{\circ}\right)$ is used to resolve the adsorption of molecules over time. The measurement spot is located at a distance $\Delta x=1 \mathrm{~mm}$ from the liquid interface, and has a diameter of approximately $1 \mathrm{~mm}$. The obtained thickness is an average over the area of the measurement spot. To obtain the thickness of the adsorbed layer we perform a measurement of the ellipsometric spectrum $\left(1.2-4.5 \mathrm{eV}, 75^{\circ}\right)$, once the dynamic measurement indicates that the adsorption has reached equilibrium. The normalised adsorption density $\Gamma / \Gamma_{\infty}$ is calculated from the thickness using $\Gamma / \Gamma_{\infty}=d / d_{\text {sat }}$. The value of $d_{\text {sat }}$, the thickness of the adsorbed film under saturated vapour conditions, is measured in a separate experiment in a closed chamber. The uncertainty in the ellipsometry measurements originates from the uncertainty in the native oxide layer thickness and the uncertainty in the Cauchy layer fit which is used to determine the adsorbed layer thickness.

The substrate on which the adsorption is measured is never in direct contact with the liquid. A similar technique was used by Novotny and Marmur [129]. This means that all measurements only take into account the molecules that are transported across the vapour phase separating the substrate and liquid. We compare the measurement with a gap (i.e., the case where no direct contact between the substrate and the liquid exists) to one without a gap (i.e., the case where direct contact between the substrate and liquid exists; the drop was placed directly on the substrate) in the appendix. Within the error margin there is no significant difference between the two measurements, indicating that the bulk of molecules adsorbed on the solid are transported across the vapour, and not, for instance, by fluid flow in a precursor film on the substrate.

\subsection{Marangoni contraction}

We first turn to the hydrodynamic mechanism, which is known as "Marangoni contraction" [19]. Some multi-component drops (e.g., water-1,2-propanediol (1,2-PrD) mixtures) can form non-zero contact angles on high-energy surfaces, even though the individual liquids themselves perfectly wet the surface at equilibrium (i.e. $\left.\theta=0^{\circ}\right)[19,115,126,127,130]$. There are two requirements that need to be satisfied for Marangoni contraction to occur: (i) one of the two liquids must be significantly more volatile than the other and (ii) the least volatile liquid should have the lowest surface tension of the two liquids. 
Selective evaporation at the contact line (where the evaporative flux is highest [28]) of the volatile component (typically water) then leads to a composition gradient in the drop and a surface tension gradient across the drop's interface. This in turn drives a Marangoni flow towards the centre of the drop, which opposes the spreading of the drop, such that the drop is "contracted". The presence of Marangoni contraction invalidates Young's law, which only holds at equilibrium, that is, in the absence of flow $[16,69]$, and its effect is opposite to Marangoni spreading [131].

Water-1,2-HD mixtures are expected to contract, since 1,2-HD is considerably less volatile than water [24], and has a surface tension lower than that of water, see Fig. 4.1c. Figure 4.3a shows the flow field inside a $\phi=0.08$ drop, as measured using high-resolution micro-particle image velocimetry. The blue line indicates the outer surface of the drop, and the contact line is located at $y=0$. A strong inward flow exists near the surface of the drop, while an outward flow towards the contact line is observed in the bulk of the drop. This flow field is typical for Marangoni-contracted drops [19]. To further test the hypothesis that the increase of $\theta$ is due to Marangoni contraction, we varied the relative humidity $(\mathrm{RH})$. A low $\mathrm{RH}$ enhances the evaporation that drives the flow inside the drop [132]. Indeed, Fig. 4.1a shows that with a lower RH, the increase of $\theta$ is significantly enhanced, and for small $\phi$ our data follows the Marangoni contraction scaling law (see the appendix) [19]. Therefore, we conclude that Marangoni contraction is responsible for the enhanced contact angle of water-1,2-HD drops at a small $\phi$.

Marangoni contraction alone, however, cannot explain the full range of data in Fig. 4.1a. At $\phi=1$ all surface tension gradients are removed, but nevertheless a large (non-zero) $\theta$ is observed. Furthermore, a monotonic increase of $\theta$ with $\phi$ is observed in Fig. 4.1a, even though a decrease in $\theta$ is expected for $\phi \gtrsim 0.6$, as is the case for $1,2-\mathrm{PrD}$ which has been shown to contract due to smaller surface tension gradients and weaker internal flow [19,127]. Figure $4.3 \mathrm{~b}$ shows the velocity field in a drop at $\phi=0.22$, which is almost one order of magnitude smaller than the velocity in the $\phi=0.08$ drop, which is too weak to sustain a contracted drop.

\subsection{Autophobing}

Another mechanism must be responsible for the large $\theta$ measured for a large $\phi$. We recall the surfactant-like nature of 1,2 -HD molecules. Some surfactantcontaining liquids are known to be autophobic on selected substrates, a phe- 


\subsection{Autophobing}

nomenon where $\theta$ increases due to modification of the solid surface energy by a precursor of adsorbed surfactant molecules [133-138]. This layer of adsorbed molecules, which is of (quasi)monolayer thickness, is of different origin than the liquid precursor that is observed in "regular" wetting [113]. The surface energy of a precursor depends on $\mathrm{RH}$, the composition of the drop, and the molecular nature of the adsorbing molecules $[116,139,140]$. To the best of our knowledge, autophobing and Marangoni contraction have never been reported to compete in a single multi-component system. Importantly, the apparent shapes of the drops is indistinguishable between the two states, but their dynamic behaviour, especially their mobility and internal flows, is very different [127].

To induce autophobing, surfactant molecules have to adsorb on the solidliquid interface (inside the drop) or on the solid-vapour interface (the precursor outside the drop), resulting in an overall decrease of $\gamma_{\mathrm{SV}}-\gamma_{\mathrm{SL}}$, where $\gamma_{\mathrm{SV}}$ is the surface tension of the solid-vapour interface, and $\gamma_{\mathrm{SL}}$ is the surface tension of the solid-liquid interface. In Fig. 4.4a we report the adsorption properties of water-1,2-HD mixtures on the solid-vapour interface under ambient conditions, measured using ellipsometry [129]. Here, $\Gamma$ is the number density of adsorbed 1,2-HD molecules, which we normalise by $\Gamma_{\infty}$, the number density of adsorbed molecules corresponding to saturated coverage (measured in a closed chamber with saturated $1,2-\mathrm{HD}$ vapour). All values of $\Gamma / \Gamma_{\infty}$ were obtained after equilibrium was reached, as determined by measuring the temporal evolution of the adsorbed layer (Fig. 4.4b), typically within a few minutes after deposition of the liquid. Complete desorption of the precursor upon removal of the drop typically takes an order of magnitude longer than the time it takes for the precursor to form, see Fig. 4.4c.

s Figure 4.4a shows clear evidence of the adsorption of 1,2-HD molecules on the substrate. Additionally, it shows that $\Gamma / \Gamma_{\infty}$ decreases both with the distance to the contact line $\Delta x$ and with $\phi$. This indicates that the concentration of 1,2-HD in the vapour surrounding the drop is of key importance to the equilibrium surface concentration of molecules adsorbed on the substrate. As we increase $\Delta x$ or decrease $\phi$, the concentration of $1,2-\mathrm{HD}$ molecules in the vapour decreases. Hence, a lower number of 1,2-HD molecules is available in the vapour to adsorb on the substrate, while water becomes more abundant. Therefore, water coverage increases with increasing $\Delta x$ and decreasing $\phi$, resulting in a lower $\Gamma / \Gamma_{\infty}$.

This indeed offers a direct explanation of the result in Fig. 4.1a, even when $\phi>\phi_{\mathrm{cmc}}$, where $\theta$ increases with $\phi$ and decreases with RH. An increase 

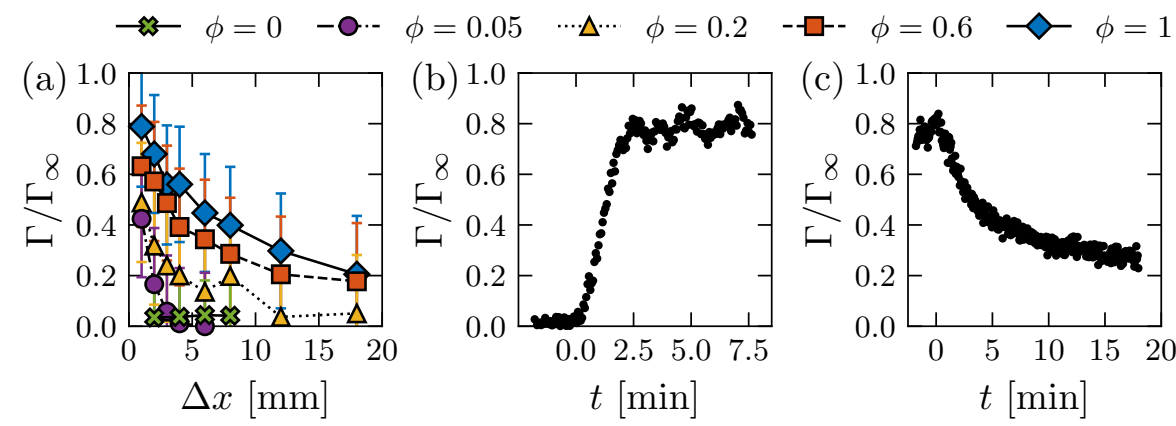

Figure 4.4: (a) Normalised adsorption density $\left(\Gamma / \Gamma_{\infty}\right)$ as a function of distance to the contact line $(\Delta x)$ for several water-1,2-HD mixtures. (b) Temporal adsorption dynamics of pure $1,2-\mathrm{HD}$ at $\Delta x \approx 5 \mathrm{~mm}$. The liquid is deposited at $t=0$. (c) Temporal desorption dynamics of pure 1,2-HD at $\Delta x=5 \mathrm{~mm}$. The container with liquid is removed at $t=0$, after which an immediate change in $\Gamma / \Gamma_{\infty}$ is visible. Complete desorption of the adsorbed molecules takes at least one order of magnitude longer than adsorption.

in $\mathrm{RH}$ leads to a lower $\Gamma / \Gamma_{\infty}$ due to the increased water coverage. Conversely, the 1,2-HD coverage increases by increasing $\phi$. The adsorbed molecules change the surface energy of the substrate, making it more hydrophobic [141]. This offers clear and direct evidence that the contact angles of autophobed drops depend on the $\mathrm{RH}$ of the close surrounding of the contact line. We remind that the internal flow is very weak at large $\phi$ (Fig. 4.3b), for which we thus expect to recover the true equilibrium contact angle. In Young's law, which remains valid at equilibrium in the presence of surfactants [138], the increased hydrophobicity of the substrate is reflected in the $\gamma_{\mathrm{SV}}-\gamma_{\mathrm{SL}}$ term, which becomes smaller with increasing $\Gamma / \Gamma_{\infty}$. Consequently, $\theta$ must increase, even though $\gamma_{\mathrm{LV}}$ remains constant above the cmc. This mechanism is reminiscent of the "modified Young's law" modelling approach used for multi-component drops in Refs. [126] and [127]. Molecules may also adsorb on the solid-liquid interface, which we are unable to measure using our experimental setup [142]. Such adsorption, if dominant, could lower $\gamma_{\mathrm{SL}}$, increase $\gamma_{\mathrm{SV}}-\gamma_{\mathrm{SL}}$, and thus lead to a decrease in $\theta$. The increase of $\theta$ and the strong dependence of $\theta$ on RH (Fig. 4.1a) indicate that adsorption on the solid-vapour interface is dominant over adsorption on the solid-liquid interface, leading to a decrease in $\gamma_{\mathrm{SV}}-\gamma_{\mathrm{SL}}$ and an increase in $\theta$ at large $\phi$.

Contrary to many previous works on autophobing [141-147], we do not 


\subsection{Effect of the molecular structure}

see an initial spreading phase followed by a retraction to the quasi-steady $\theta$ (see Fig. 4.1c). This is likely due to the relatively high diffusion coefficient of $1,2-\mathrm{HD}$, which is a result of its small molecular size in comparison to other more common surfactants [148]. The region of the substrate that is sampled by the liquid in determining the stationary $\theta$ is no larger than $10 \mu \mathrm{m}$ [149]. The timescale associated with forming the equilibrium adsorption layer within this region is smaller than the spreading timescale [150], which is relatively long due to the high viscosity of $1,2-\mathrm{HD}(\eta \approx 82 \mathrm{mPa} \cdot \mathrm{s}[151])$.

\subsection{Effect of the molecular structure}

Our experiments show that water-1,2-HD mixtures exhibit a competition between Marangoni contraction and autophobing. How generic is the observed competition between Marangoni contraction and authophobing and what is the influence of the surface activity $\mathrm{d} \gamma_{\mathrm{LV}} / \mathrm{d} \phi$ ? Here, we address these questions by considering three shorter vicinal alkanediols: 1,2-PrD), 1,2-butanediol (1,2-BD), and 1,2-pentanediol (1,2-PeD), which have three, four, and five carbon atoms in their aliphatic chain, respectively. These diols are non-volatile and have a low $\gamma_{\mathrm{LV}}$ [64]. The surfactant-like behaviour (i.e., the surface activity $\mathrm{d} \gamma_{\mathrm{LV}} / \mathrm{d} \phi$ ) depends on the length of the aliphatic chain. Short chain alkanediols show weaker surfactant-like behaviour (smaller $\mathrm{d} \gamma_{\mathrm{LV}} / \mathrm{d} \phi$ ) due to the decreased hydrophobicity of the molecule $[64,152]$.

We study the properties of these diols using the same procedure as we used for 1,2-HD. Figure 4.5a shows $\theta$ as a function of $\phi$ at $\mathrm{RH} \approx 60 \%$. Starting at small $\phi$, we see that all diols follow a universal curve. This is perfectly consistent with Marangoni contraction, as long as $\mathrm{d} \gamma_{\mathrm{LV}} / \mathrm{d} \phi$ is sufficiently smaller than zero, and water remains more volatile in the mixture; the hydrodynamic mechanism remains insensitive to molecular details, while absolute flow velocities depend on the material parameters. Mixtures of other liquids are also expected to contract as long as their volatility and surface tension contrasts are in the same regime as those of water and carbon diols [126]. By contrast, the curves start to diverge and the length of the aliphatic chain matters for larger $\phi$ - consistent with autophobing. The longest diol studied here, 1,2-HD, exhibits strong autophobing behaviour. As we move to short chain diols, the autophobing strength becomes smaller, indicated by smaller values of $\theta$ at $\phi=1$. Additionally, Fig. 4.5a shows that Marangoni contraction is the dominant mechanism up to a larger $\phi$ for shorter diols. While autophobing is dominant for 1,2 -HD starting from $\phi \approx 0.3$, for 1,2 -PrD, by contrast, the full 

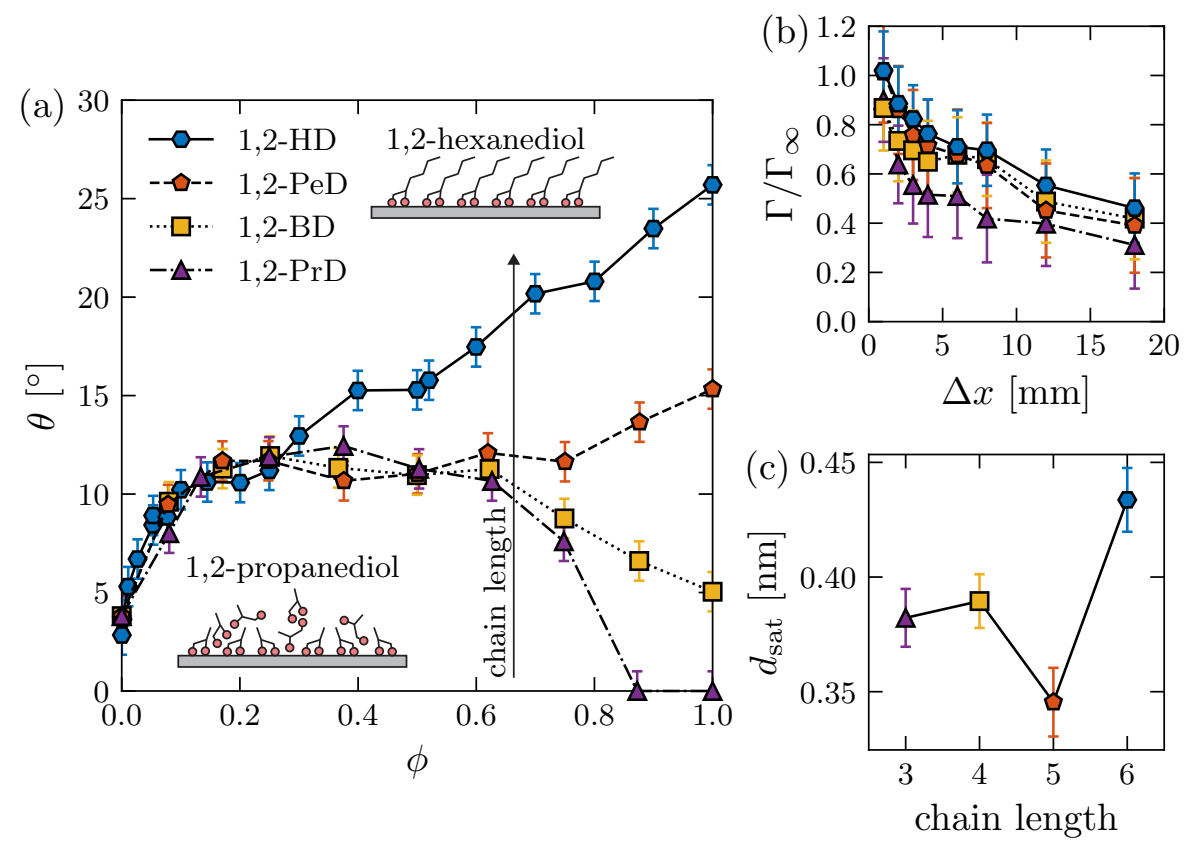

Figure 4.5: (a) Contact angle $(\theta)$ as a function of mass fraction $(\phi)$ for several mixtures of water and vicinal alkanediols $(\mathrm{RH}=60 \%)$. The schematics show the structure of adsorbed 1,2-PrD molecules and 1,2-HD molecules. (b) Normalised adsorption density $\left(\Gamma / \Gamma_{\infty}\right)$ as a function of distance to the contact line $(\Delta x)$. (c) Thickness of the saturated film $\left(d_{\text {sat }}\right)$ for several vicinal alkanediols.

range of $\phi$ is consistent with Marangoni contraction - there is no autophobing at all. Hence, a higher surface activity does not necessarily lead to stronger Marangoni contraction. In fact, the surface activity of the molecules may inhibit contraction, leading to autophobed drops. For example, at large $\phi$, 1,2-HD (highest $\mathrm{d} \gamma_{\mathrm{LV}} / \mathrm{d} \phi$ ) shows the strongest autophobing, whereas 1,2-PrD (lowest $\mathrm{d} \gamma_{\mathrm{LV}} / \mathrm{d} \phi$ ) drops are contracted. Thus, our results show that, in addition to the two requirements listed above, there is a third requirement that needs to be satisfied for drops to contract: the contact angle achievable by Marangoni contraction needs to be larger than the microscopic contact angle as governed by molecular forces. However, the microscopic angle may be larger than zero.

All four molecules adsorb on the substrate, as seen from the ellipsometry 


\subsection{Conclusion and outlook}

measurements presented in Fig. 4.5b. The reduced autophobing strength of the shorter diols is caused by the shorter hydrophobic chain in these molecules. The distance between the hydrophilic and hydrophobic parts of the molecule is smaller in shorter chain molecules, meaning that the polar nature of the hydroxyl groups becomes more relevant for the surface energy of an adsorbed layer of a short chain molecule such as $1,2-\mathrm{PrD}$. The result is a more hydrophilic surface and therefore a smaller $\theta$. Figure $4.5 \mathrm{~b}$ shows that all diols studied here adsorb onto the substrate with similar $\Gamma / \Gamma_{\infty}$. However, as shown in Fig. 4.5c, not all adsorb in the same way as 1,2-HD. Despite their smaller size, the saturated thickness $d_{\text {sat }}$ of $1,2-\mathrm{PrD}$ and $1,2-\mathrm{BD}$ is larger than that of $1,2-\mathrm{PeD}$ and only slightly smaller than that of $1,2-\mathrm{HD}$, suggesting that they do not form monolayers (an estimate of the size of each molecule is given in the appendix), since a monotonic increase in $d_{\text {sat }}$ with the chain length is expected if monolayers are formed. Their hydroxyl groups remain partially exposed, allowing them to form disordered multi-layered structures (see the schematic in Fig. 4.5a) similar to layers of adsorbed water molecules [153]. Hence, they do not strongly affect the surface energy. By contrast, 1,2-PeD and 1,2-HD likely adsorb in a monolayer structure (see the schematic in Fig. 4.5a), indicated by the increasing $d_{\text {sat }}$ between 1,2-PeD and 1,2-HD in Fig. 4.5c and the decrease in $d_{\text {sat }}$ between $1,2-\mathrm{PrD}$ and $1,2-\mathrm{PeD}$. This means that their long aliphatic chains are exposed, increasing the hydrophobicity of the surface. Therefore, autophobing occurs at large $\phi$ for molecules with a long aliphatic chain due to the strong effect of the adsorbed molecules on the surface energy of the solid. By contrast, adsorbed molecules with a short aliphatic chain have little effect on the surface energy of the solid, and Marangoni contraction dominates over the full range of $\phi$. One can thus tune $\theta$ over a large range by selecting the correct diol and a particular combination of $\phi$ and $\mathrm{RH}$.

\subsection{Conclusion and outlook}

Our results reveal that Marangoni contraction and autophobing both provide valid descriptions for the wetting of two-component drops, albeit in different regimes. A minute change in one of the control parameters is sufficient to change the dominant wetting mechanism. While the visual appearance of drops in either of the two wetting states is indistinguishable, Figure 4.3 demonstrates a strong difference in their internal flows. We have shown (Fig. 4.1a and 4.5a) that Marangoni contraction is possible only if the microscopic contact angle, as governed by molecular forces, is smaller than the angle achievable by 
contraction. Additionally, we show (Fig. 4.3) that the internal flows should be used to determine the state of a drop rather than the contact angle or the apparent drop shape. By systematically changing the molecular structure of the volatile liquid, we show that a higher surface activity $\mathrm{d} \gamma_{\mathrm{LV}} / \mathrm{d} \phi$ does not necessarily lead to stronger Marangoni contraction. In fact, excessive surface activity may inhibit contraction and lead to drops whose contact angle is governed by molecular forces. Hence, the chemical structure of the liquid needs to be taken into account when designing multi-component drop systems with specific properties. Importantly, these mechanisms are generic and expected to be present in most mixtures containing (volatile) surfactant-like liquids (e.g., single alcohols).

Marangoni-contracted drops are attractive for technological applications due to their high mobility $[126,127,154]$, which is suppressed for drops in the autophobing or partial wetting states. Our result may also be of interest to applications that require high contact angles of drops consisting of low surface tension liquids, such as inkjet printing [8] or semiconductor processing [115]. In the context of inkjet printing our results are particularly relevant to nozzle wetting (see Fig. 1.10d). Marangoni flow of ink on the nozzle of a printhead often leads to print failure, since the nozzle is unable to reliable jet ink drops. Non-wetting inks have been suggested as a remedy for this problem [26]. Our results may be useful for the selection of the components of such inks. 


\subsection{Appendix}

\subsection{Appendix}

\subsubsection{Ellipsometry measurements}
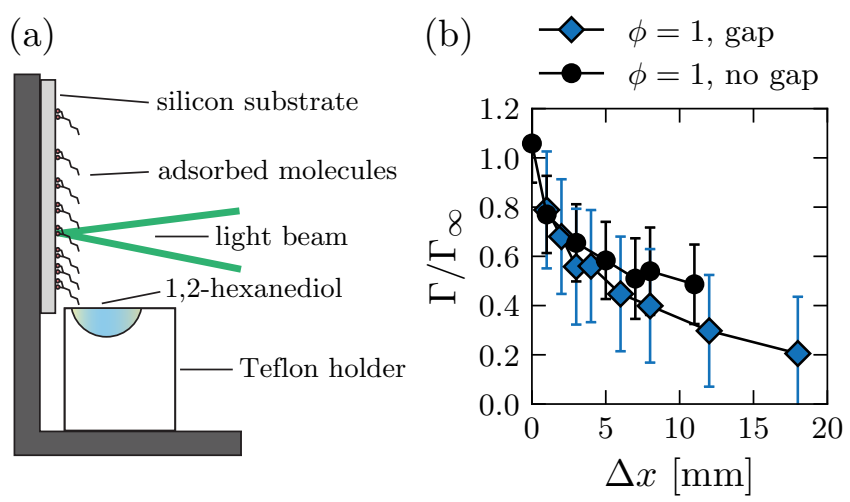

Figure 4.6: (a) Ellipsometry setup. The substrate is mounted vertically, and is not in direct contact with the liquid (here: 1,2-hexanediol). The adsorption of molecules is measured at a small distance from the liquid's interface. (b) Adsorption close to pure 1,2-hexanediol drops. A 'gap' means that the substrate on which the ellipsometry measurement is performed is not in direct contact with the liquid (as sketched in (a)). Conversely, 'no gap' means that there is direct contact between the liquid and the substrate.

\subsubsection{Marangoni contraction scaling law}

Figure 4.7a shows all data from Fig. 4.1a and Fig. 4.5a of the main text scaled according to the scaling law $\theta(\phi) \propto\left(\mathrm{RH}_{\mathrm{eq}}(\phi)-R H\right)^{1 / 3}$ (i.e. Eq. 1 from [19]). Figure $4.7 \mathrm{~b}$ shows $\mathrm{RH}_{\mathrm{eq}}$, the relative humidity at which a drop spreads completely (i.e., $\theta=0^{\circ}$ ), as determined by a model equation (Eq. 7 from [19]), which is used in Fig. 4.7a. All data points below the red line (i.e., $R H_{\text {eq }}-R H=0$ ) in Fig. 4.7c indicate experiments where the contact angle should be zero if the contact angle is determined by Marangoni contraction, yet, for most of these experiments, the contact angle is larger than zero due to autophobing (c.f. Fig. 4.1a and Fig. 4.5a). This prevents us from experimentally determining $\mathrm{RH}_{\text {eq }}$. The key point of Fig. 4.7 is the excellent collapse of the data for $\phi \lesssim 0.2$ in Fig. 4.7a, indicating that Marangoni contraction is the dominant mechanism for these mass fractions. 


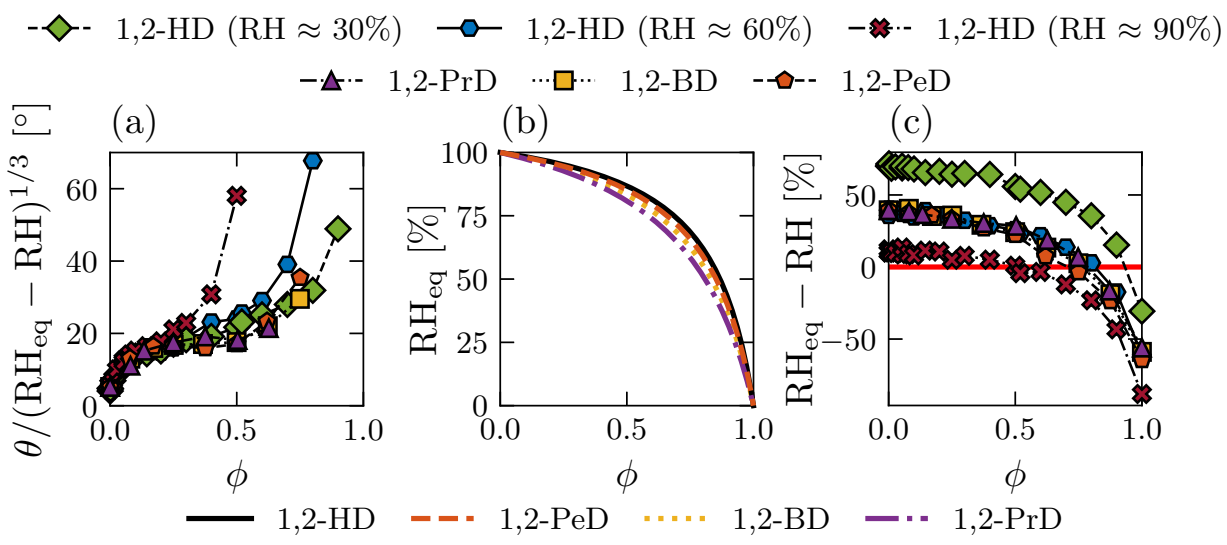

Figure 4.7: (a) Marangoni contraction scaling law (Eq. 1 from [19]) applied to the data in (a). Note the excellent collapse for small $\phi$. (b) The relative humidity at which the drop spreads completely $\left(\mathrm{RH}_{\mathrm{eq}}\right)$ as a function of mass fraction as determined by the model (Eq. 7 from [19]). (c) Difference between $\mathrm{RH}_{\mathrm{eq}}$ and $\mathrm{RH}$ as a function of mass fraction. Any data point below the red line (i.e., $R H_{\mathrm{eq}}-R H=0$ ) should spread completely (i.e., $\theta=0^{\circ}$ ), but autophobing prevents this.

\subsubsection{Estimate of the molecular length}

The length of a molecule can be estimated using

$$
l=\left(\frac{M}{\rho N_{A}}\right)^{\frac{1}{3}},
$$

where $M$ and $\rho$ are the molar mass and density of the molecule, and $N_{A}$ is the Avogadro constant $\left(6.02214076 \times 10^{23}\right)$. An overview of the properties and resulting molecular lengths is shown in Table 4.1. Note that these lengths are approximations - the molecular length depends strongly on the confirmation of the molecule, which is influenced by the surroundings of the molecule; this is not accounted for in Eq. 4.2. 
4.7. Appendix

\begin{tabular}{l|c|c|c} 
molecule & $M[\mathrm{~g} / \mathrm{mol}]$ & $\rho\left[\mathrm{kg} / \mathrm{m}^{3}\right]$ & $l[\AA]$ \\
\hline 1,2-propanediol & 76.09 & 1036 & 4.96 \\
1,2-butanediol & 90.12 & 1006 & 5.30 \\
1,2-pentanediol & 104.15 & 971 & 5.63 \\
1,2-hexanediol & 118.17 & 951 & 5.91
\end{tabular}

Table 4.1: Properties of several alkane diols and the resulting estimated molecule lengths obtained using Eq. 4.2. 



\section{Part II}

\section{Coalescence}





\section{Chapter 5}

\section{Self-similar liquid lens coalescence}

A basic feature of liquid drops is that they can merge upon contact to form a larger drop. In spite of its importance to various applications, drop coalescence on pre-wetted substrates has received little attention. Here, we experimentally and theoretically reveal the dynamics of drop coalescence on a thick layer of a low viscosity liquid. It is shown that these so-called "liquid lenses" merge by the self-similar vertical growth of a bridge connecting the two lenses. Using a slender analysis, we derive similarity solutions corresponding to the viscous and inertial limits. Excellent agreement is found with the experiments without any adjustable parameters, capturing both the spatial and temporal structures of the flow during coalescence. Finally, we consider the crossover between the two regimes, and show that all data of different lens viscosities collapse on a single curve capturing the full range of the coalescence dynamics.

๒ Published as: Michiel A. Hack, Walter Tewes, Qingguang Xie, Charu Datt, Kirsten Harth, Jens Harting, and Jacco H. Snoeijer, Self-Similar Liquid Lens Coalescence, Phys. Rev. Lett. 124, 194502 (2020). 


\subsection{Introduction}

The coalescence of liquid drops is an important part of many industrial processes, such as inkjet printing and lithography $[6,8]$. It is also ubiquitously observed in nature, for example, in the formation of rain drops and the selfcleaning of plant leaves [155-157]. Coalescence, therefore, has been the focus of many studies, primarily for spherical drops [45, 57-59,61], but also for drops on a solid substrate $[42,44,48,50,158]$. In contrast, little work exists on the coalescence of drops on liquid substrates, despite its importance for emerging applications such as fog harvesting, anti-icing, wet-on-wet printing, enhanced oil recovery, emulsions, and wetting of lubricant-impregnated surfaces $[25,35,79,86,159-166]$.

The dynamics of coalescence are strongly affected by the geometry of the drops. Drops on a solid substrate (spherical caps) merge differently than freely suspended drops (axisymmetric spheres), with different scaling exponents for the growth of the bridge between the drops [42, 44, 45, 48, 50, 57-59, 61, 158]. This is in contrast to the coalescence of drops floating on a liquid substrate (Fig. 5.1a); such drops are referred to as "liquid lenses" [69, 167]. For coalescing lenses, the growth of the bridge width based on a top-view experiment was found similar to that of axisymmetric drops, which is surprising since, geometrically, liquid lenses are spherical caps [35].

In this chapter, we study the coalescence dynamics of liquid lenses in terms of the vertical bridge growth $h_{0}(t)$ (defined in Fig. 5.1a) and reveal a strong departure from the coalescence of axisymmetric drops. We first experimentally establish the initial dynamics of coalescence of drops of varying viscosity from the side-view perspective, identifying two distinct regimes - one dominated by viscosity and the other by inertia. Subsequently, we develop a fully quantitative slender description for each of these regimes based on the self-similar nature of coalescence. In the spirit of recent work on spherical drops, we identify the master curve for all data, including the crossover between the two regimes $[45,54]$. Unlike for any other coalescence problem, however, the master curve here is obtained without any adjustable parameter.

\subsection{Experimental methods}

Two small drops are placed on a deionised water surface (MilliQ, Millipore Corporation) kept in a large container. The lenses consist of mineral oils (RTM series, Paragon Scientific Ltd.), with viscosities between $\eta=18 \mathrm{mPa} \cdot \mathrm{s}$ 


\subsection{Experimental methods}

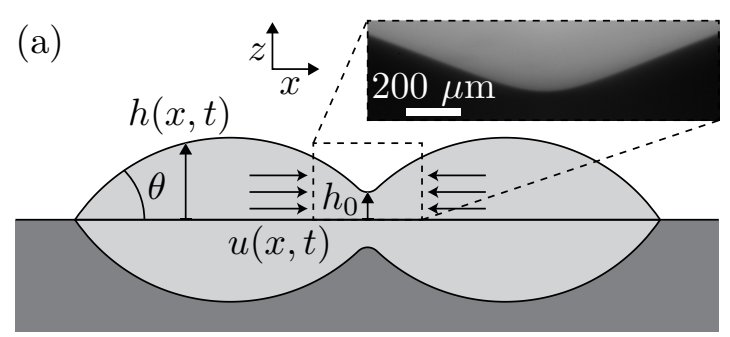

(b)

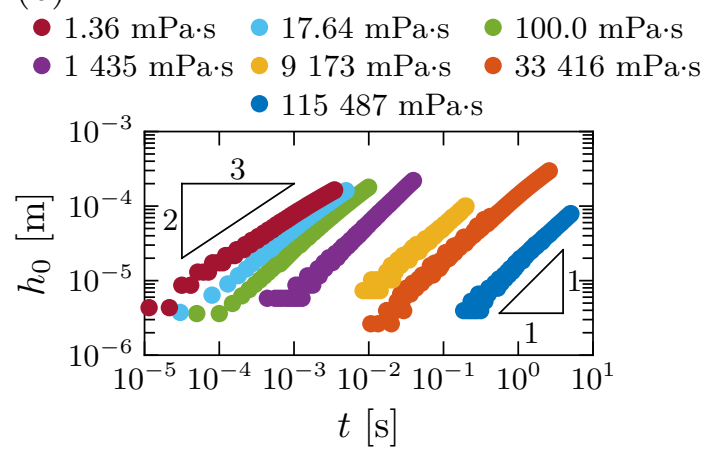

Figure 5.1: (a) Schematic view of two coalescing liquid lenses connected by a bridge of height $h_{0}(t)$. The lenses float on a pool with a depth that is much larger than the size of the lenses. The zoomed region shows a typical snapshot of the bridge region. (b) Measurements of the bridge height $h_{0}$ as a function of time $t$ for several viscosities. Two distinct power laws are identified.

and $115 \mathrm{~Pa} \cdot \mathrm{s}$ and surface tension $\gamma=34 \mathrm{mN} / \mathrm{m}$ (measured by the pendant drop method [100]). Additionally, we use dodecane lenses (Sigma-Aldrich, $\eta=1.36 \mathrm{mPa} \cdot \mathrm{s}, \gamma=25 \mathrm{mN} / \mathrm{m})$. These liquids float on the water surface since their densities $\left(\rho=850 \mathrm{~kg} / \mathrm{m}^{3}\right.$ for mineral oil and $\rho=750 \mathrm{~kg} / \mathrm{m}^{3}$ for dodecane) are lower than the density of water $\left(\rho=997 \mathrm{~kg} / \mathrm{m}^{3}\right)$. Both liquids have a negative spreading parameter, and thus form lenses with small but finite contact angles $\theta=26^{\circ}-37^{\circ}[69]$. Since the contact angle of the oil-water interface is within $5^{\circ}$ of the aforementioned values, we regard the lenses as being top-down symmetric.

We image the coalescing lenses from the side using a high-speed camera (Photron Nova S12) equipped with a microscopic lens (Navitar $12 X$ zoom lens). In order to obtain a sharp image of the oil-air interface of the liquid lens, the container of the pool is filled such that a convex meniscus forms at 
the edges of the container. Frame rates between 250 and 100000 frames/s are used depending on the timescale of coalescence, with resolutions in the range of $1.3-5.3 \mu \mathrm{m} /$ pixel. A typical snapshot of the bridge region is shown in the zoomed region in Fig. 5.1a.

The experiment is performed as follows: two pendant drops with volume $V=2.5 \mu \mathrm{L}$ are formed on two identical blunt-ended metal needles using a syringe pump (we have verified that drop size does not affect the initial coalescence dynamics, as we will show below). Using a linear translation stage, the drops are gently brought into contact with the water pool and subsequently form lenses of radius $R \approx 2.5 \mathrm{~mm}$. The lenses are left to equilibrate for a moment before the syringes are gently removed. Capillary interactions drive the lenses toward each other and they coalesce upon first contact. We define $t=0$ as the first frame where the bridge connecting the two lenses is visible, and $h=0$ at the surface of the pool. The velocity of the approaching lenses is orders of magnitude smaller than the velocity of the bridge growth.

\subsection{Coalescence dynamics}

The experiments reveal that the coalescence of liquid lenses is governed by a self-similar power-law growth of the bridge that connects the two drops. Figure $5.1 \mathrm{~b}$ shows the minimum bridge height $h_{0}$ as a function of time after contact $t$ for coalescing lenses of different viscosities. We clearly distinguish two regimes: a nonlinear regime for small viscosities where $h_{0} \propto t^{2 / 3}$, and a linear regime where $h_{0} \propto t$ for high viscosities. These exponents are typical for pinch-off and coalescence of spherical caps on a solid substrate, though the prefactors are different; lens coalescence is much faster as will be discussed more quantitatively below $[36,44,50,168]$. These growth dynamics, however, are different from those of spherical drops and of those observed for lenses in topview [35]. To further investigate this, we now first focus on the case of viscous coalescence. Figure 5.2a shows the temporal evolution of the bridge, which grows at constant velocity. The bridge velocity decreases when $h_{0}$ becomes of the order of the lens size, due to the finite height $(\approx 0.5 \mathrm{~mm})$ of the lens. The spatial structure of coalescence is revealed in Fig. 5.2b, where we compare the shape of the bridge at various times. We scale the horizontal and vertical coordinates by $h_{0}$, which is presumably the only relevant length scale in the problem, and observe an excellent collapse of the data. This implies that the bridge growth exhibits self-similar dynamics, that we now set out to describe analytically. 
(a)

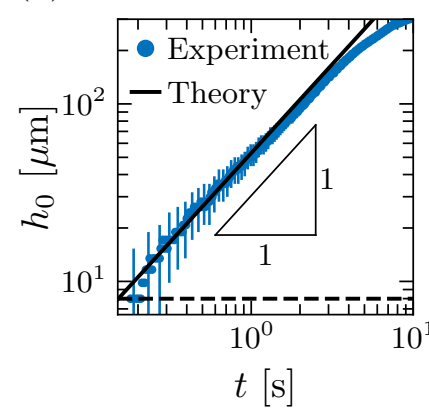

(b)

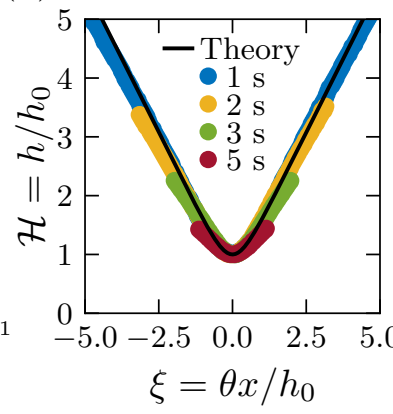

(c)

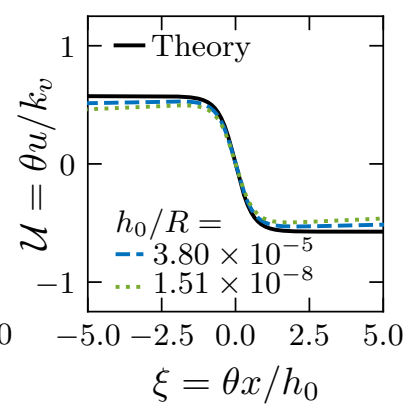

Figure 5.2: Coalescence in the viscous regime. (a) Height of the bridge $h_{0}$ as a function of time after contact $t$ (mineral oil lenses, $\theta=33^{\circ}$, $\eta=115487 \mathrm{mPa} \cdot \mathrm{s}$, initial height $\approx 0.5 \mathrm{~mm})$. The solid line is the prediction from Eq. 5.6. The error bars are only shown for one in every ten datapoints for clarity. The horizontal dashed line indicates the resolution limit. (b) Rescaled experimental profiles at different times, $\mathcal{H}=h(x, t) / h_{0}(t)$ versus $\xi=x \theta / h_{0}(t)$. The collapse of the profiles indicates self-similar dynamics. The solid line is the similarity solution obtained from Eqs. 5.4-5.5. (c) Rescaled velocity profile. The solid line is the similarity solution. The coloured lines are numerical simulations for different values of $h_{0} / R$.

\subsection{Viscous and inertial similarity solutions}

The main assumptions of our analysis are that (i) the flow during the initial stage of coalescence is predominantly parallel to the $x z$ plane (rendering the problem two-dimensional, following, e.g., $[42,44,158]$ ) and (ii) the limiting mechanism for coalescence is the flow inside the drops (i.e.. negligible flow inside the sub-phase, which in all but one experiment is at least one order of magnitude less viscous than the drop). Then, we can make use of the slender geometry of the system and use the thin-sheet equations,

$$
\begin{aligned}
h_{t}+(u h)_{x} & =0, \\
\rho\left(u_{t}+u u_{x}\right) & =\gamma h_{x x x}+4 \eta \frac{\left(u_{x} h\right)_{x}}{h},
\end{aligned}
$$

which represent mass conservation and momentum conservation, respectively [169-171]. Here, $h(x, t)$ is the shape of the bridge (Fig. 5.1a) and $u(x, t)$ is the horizontal velocity of the liquid inside the lenses (which is a plug flow to leading 
order in the slender approximation). The shape of the lens is assumed to be top-down symmetric, with uncertainty owing to the weak differences in surface tensions estimated to be less than 10\% (see the appendix). We therefore take $\gamma$ as the surface tension of the lenses with respect to the surrounding air. The effect of gravity is expected to be negligible because the bridge is initially much smaller than the capillary length $\lambda_{c}=\sqrt{\gamma /(\Delta \rho g)}=\mathcal{O}(1) \mathrm{mm}$, and therefore we exclude it from the analysis. Encouraged by the experiments, we search for similarity solutions of the form

$$
h(x, t)=k t^{\alpha} \mathcal{H}(\xi), \quad u(x, t)=\frac{\alpha k}{\theta} t^{\beta} \mathcal{U}(\xi), \quad \xi=\frac{\theta x}{k t^{\alpha}},
$$

where $\mathcal{H}$ and $\mathcal{U}$ are the similarity functions for the bridge profile and flow velocity. The choice of $\xi$ ensures that $h(x, t) \simeq \theta x$ far away from the bridge, in order to match a static solution with a contact angle $\theta$.

We first examine the viscous regime, by setting $\rho \approx 0$ in Eqs. 5.1-5.2. Inserting Eq. 5.3 then readily leads to $\alpha=1$ and $\beta=0$, and explains the linear growth observed in the experiment. The parameter $k=k_{v}=\mathrm{d} h_{0} / \mathrm{d} t$ thus provides the dimensional bridge velocity and will be computed below. Equations 5.1-5.2 further reduce to

$$
\begin{aligned}
\mathcal{H}-\xi \mathcal{H}^{\prime}+(\mathcal{H} \mathcal{U})^{\prime} & =0 \\
\mathcal{H} \mathcal{H}^{\prime \prime \prime}+K_{v}\left(\mathcal{U}^{\prime} \mathcal{H}\right)^{\prime} & =0
\end{aligned}
$$

providing a fourth order system of ODEs, that contains a parameter

$$
K_{v}=\frac{4 \eta k_{v}}{\gamma \theta^{2}}
$$

representing the dimensionless bridge velocity. Hence, the selection of a unique solution requires five boundary conditions. We consider symmetric solutions and normalise the bridge height to unity at $\xi=0$, so that

$$
\mathcal{H}(0)=1, \quad \mathcal{H}^{\prime}(0)=0, \quad \text { and } \quad \mathcal{U}(0)=0 .
$$

At large scale, this solution should match an initially static drop. This implies that the leading order asymptotics for large $\xi$ of $\mathcal{H}, \mathcal{U}$ must correspond to time-independent $h, u$. For the bridge profile, this implies $\mathcal{H}^{\prime}(\infty) \rightarrow 1$, where we have also used the matching to the contact angle $\theta$. The velocity to leading order is $\mathcal{U} \simeq C \log \xi$ as $\xi \rightarrow \infty$; recalling that $\xi \sim x / t$, a static drop at $t=0$ corresponds to $C=0$, which provides the fifth boundary condition. The 


\subsection{Viscous and inertial similarity solutions}

resulting boundary value problem is solved numerically by a shooting method, resulting in $K_{v}=2.210$. In chapter 6 we further study the viscous case, and show that, depending on the the large-scale boundary conditions at $x=R$, significant corrections to the similarity solution may arise.

We find excellent agreement between the experimental data and the similarity solution. The solid line in Fig. 5.2a corresponds to the velocity prediction Eq. 5.6 without any adjustable parameters. It is of interest to compare this result to the merging of drops on a solid substrate: owing to the no-slip boundary condition on a solid, coalescence is much slower on solid substrates with coalescence velocity $\sim \theta^{4}$ instead of $\sim \theta^{2}$ observed for lenses [44]. As an example, lens coalescence is approximately five times faster than coalescence on a solid substrate for the parameters of the experiment shown in Fig. 5.2a. In Fig. $5.2 \mathrm{~b}$ we compare the rescaled bridge profiles to $\mathcal{H}(\xi)$, shown as the solid line, and also find quantitative agreement. Figure 5.2c shows the self-similar velocity $\mathcal{U}(\xi)$. Since the velocity inside the drop cannot be extracted from our experiments, we numerically solve the time-dependent Eqs. 5.1-5.2 with $\rho=0$ using a finite element method (implemented using the library OOMPHLIB [172]) and compare the result to the similarity solution. Details of the numerical method are found in the appendix. The numerical data in Fig. 5.2c indeed collapse and converge to the predicted similarity profile as $h_{0} / R \rightarrow 0$.

The same scheme is followed for the regime where inertia dominates over viscosity, with the results outlined in Fig. 5.3. Once again, we insert Eq. 5.3, with $k=k_{i}$, in Eqs. 5.1-5.2 but now in the inviscid limit $(\eta=0)$. The exponents can then be computed as $\alpha=2 / 3, \beta=-1 / 3$, in agreement with the experiment. The momentum balance Eq. 5.2 now gives

$$
2 \mathcal{U} \mathcal{U}^{\prime}-\mathcal{U}-2 \xi \mathcal{U}^{\prime}-K_{i}^{-1} \mathcal{H}^{\prime \prime \prime}=0,
$$

with a dimensionless constant

$$
K_{i}=\frac{2}{9} \frac{\rho k_{i}^{3}}{\gamma \theta^{4}}
$$

Mass conservation is unchanged as compared to Eq. 5.4, so that we again require five boundary conditions to close the problem. As in the viscous case, four conditions follow from Eq. 5.7 and $\mathcal{H}^{\prime}(\infty) \rightarrow 1$. The fifth boundary condition again comes from the large- $\xi$ asymptotics - one finds $\mathcal{U} \simeq C \xi^{-1 / 2}$ as $\xi \rightarrow \infty$ [173]. This gives $u \simeq C x^{-1 / 2}$ which for a static outer drop at $t=0$ implies $C=0$. Numerically solving the boundary value problem then gives $K_{i}=0.106$. 
(a)

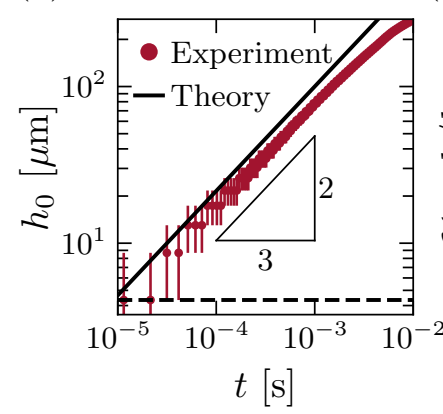

(b)

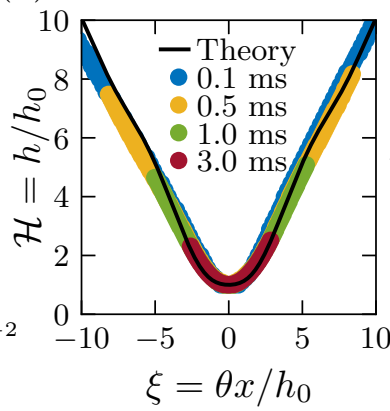

(c)

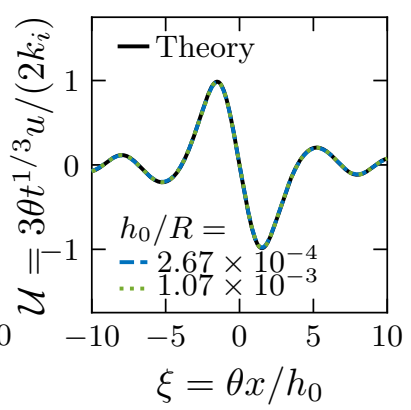

Figure 5.3: Coalescence in the inertial regime. (a) Height of the bridge $h_{0}$ as a function of time after contact $t$ (dodecane lenses, $\theta=29^{\circ}, \eta=1.36 \mathrm{mPa} \cdot \mathrm{s}$, initial height $\approx 0.5 \mathrm{~mm}$ ). The solid line is the prediction from Eq. 5.9. The horizontal dashed line indicates the resolution limit. (b) Rescaled experimental profiles at different times, $\mathcal{H}=h(x, t) / h_{0}(t)$ versus $\xi=x \theta / h_{0}(t)$. The collapse of the profiles indicates self-similar dynamics. The solid line is the similarity solution obtained from Eqs. 5.4 and 5.8. (c) Rescaled velocity profile. The solid line is the similarity solution. The coloured lines are numerical simulations for different values of $h_{0} / R$.

In Fig. 5.3a we compare Eq. 5.9 to experimental data of the lowest viscosity and find excellent agreement, without adjustable parameters. The spatial structure of the bridge also follows the predicted collapse, shown in Fig. 5.3b, and agrees with the computed form $\mathcal{H}(\xi)$ (solid line). The dimensionless velocity $\mathcal{U}(\xi)$ is again compared to numerical simulations of Eqs. 5.1-5.2 with $\eta \approx 0$, confirming the validity of the analysis. Interestingly, the velocity exhibits oscillations (Fig. 5.3c) due to coalescence-induced inertio-capillary waves $[50,56]$. These oscillations can indeed be predicted from the (higher order) asymptotics of the similarity equations [173]. However, due to the small amplitude of the waves in the similarity function $\mathcal{H}$, these oscillations are difficult to observe in the experiments. Let us remark that we cannot directly compare these results to inertial coalescence on solid substrates, since the equivalent lubrication theory is not available owing to the no-slip condition. 


\subsection{Crossover}

\subsection{Crossover}

Several coalescence events in Fig. 5.1b do not fit perfectly in either the viscous or in the inertial regime. As a final step, we therefore describe the crossover between these regimes and collapse the entire set of experimental data. An estimate of the crossover height $h_{\mathrm{c}}$ and crossover time $t_{\mathrm{c}}$ can be obtained by setting $h_{\mathrm{c}}=k_{v} t_{\mathrm{c}}=k_{i} t_{\mathrm{c}}^{2 / 3}$, from which we find

$$
h_{\mathrm{c}}=\frac{k_{i}^{3}}{k_{v}^{2}}=\frac{72 K_{i}}{K_{v}^{2}} \frac{\eta^{2}}{\rho \gamma}, \quad t_{\mathrm{c}}=\frac{k_{i}^{3}}{k_{v}^{3}}=\frac{288 K_{i}}{K_{v}^{3}} \frac{\eta^{3}}{\rho \gamma^{2} \theta^{2}} .
$$

Note that these are proportional to the intrinsic viscous scales $l_{v}=\eta^{2} /(\rho \gamma)$ and $t_{v}=\eta^{3} /\left(\rho \gamma^{2}\right)$, known for drop pinch-off, but with pre-factors coming from the similarity analysis [36]. Contrarily to pinch-off, however, we remark that the ultimate early-time coalescence is purely viscous [174].

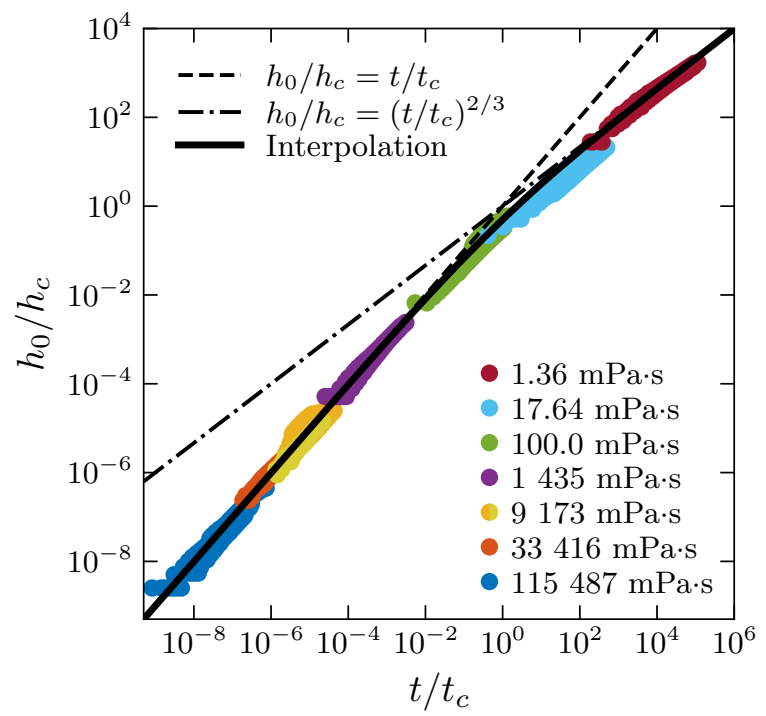

Figure 5.4: Crossover between the viscous and inertial regimes, shown by a collapse of all experimental data on a master curve. Dashed line: viscous theory. Dotted-dashed line: inertial theory. Solid line: interpolation based on Eq. 5.11. The lime-coloured data points are with larger lens size $(R \approx 4.1 \mathrm{~mm}$, compared to $R \approx 2.5 \mathrm{~mm}$ for all other data, showing that the dynamics do not depend on the drop size). 
Figure 5.4 shows coalescence events for different viscosities (varied over five orders of magnitude), made dimensionless according to the crossover scales Eq. 5.10. It is clear that the proposed scaling indeed collapses the data onto a single master curve, transitioning from the viscous to the inertial regime. Note that Fig. 5.4 also contains a data set with larger lens size, confirming that the lens size does not matter. In the spirit of the work on spherical drops and drop impact, we propose an empirical formula based on a Padé approximant which describes the two asymptotic regimes as well as the crossover region $[45,54,175]$,

$$
h_{0} / h_{\mathrm{c}}=\left(\frac{1}{t / t_{\mathrm{c}}}+\frac{1}{\left(t / t_{\mathrm{c}}\right)^{2 / 3}}\right)^{-1} .
$$

We stress that, unlike the spherical drop case, the present interpolation Eq. 5.11 contains no free parameters since $h_{c}$ and $t_{c}$ derived in Eq. 5.10 follow from the similarity solutions. The interpolation is superimposed as the solid line in Fig. 5.4, providing an accurate description for all experiments.

\subsection{Conclusion and outlook}

Our results show that the coalescence of liquid lenses is accurately described by self-similar solutions to the thin-sheet equations. We have identified the crossover between the viscous regime and the inertial regime both experimentally and analytically. These coalescence dynamics are naturally very different from axisymmetric, spherical drops, though previous top-view experiments on liquid lenses did observe axisymmetric-like dynamics - the relation between horizontal and vertical growth remains to be understood [35]. Importantly, the effect of the sub-phase viscosity is not included in our model - and apparently it plays a subdominant role for the coalescence [43]. Future work should be dedicated to more extreme cases, such as those where the viscosity of the sub-phase is much larger or where the layer thickness becomes small. This would be along the lines followed for the coalescence of circular nematic films, where the influence of dissipation in the viscous sub-phase was systematically investigated [159]. The present results provide a framework for such explorations, in particular the quantitative success of the thin-sheet equations, which will be of key interest to applications involving pre-wetted substrates. 


\subsection{Appendix}

\subsection{Appendix}

\subsubsection{Numerical simulations}

In order to verify the similarity solutions obtained from the numerical solution of the ODEs Eqs. 5.4-5.5 and Eqs. 5.4 and 5.8, we conducted numerical simulations of the time-dependent equations Eqs. 5.1-5.2. This led to the numerical velocity profiles reported in Fig. 5.2c and Fig. 5.3c. The simulations were done in non-dimensional units, where Eqs. 5.1-5.2 are written as

$$
\begin{aligned}
h_{t}+(u h)_{x} & =0, \\
\mathrm{We}\left(u_{t}+u u_{x}\right) & =h_{x x x}+4 \mathrm{Ca} \frac{\left(u_{x} h\right)_{x}}{h} .
\end{aligned}
$$

For the non-dimensionalisation, we rescaled both the height field $h$ and the $x$-coordinate by the height $h_{l}=\theta R / 2$ of the lens ( $R$ being the radius and $\theta$ the contact angle), the velocity by a typical velocity scale $U_{l}$, and time accordingly by a scale $h_{l} / U_{l}$. Furthermore, we introduced the non-dimensional Weber number $\mathrm{We}=\rho U_{l}^{2} h_{l} / \gamma$ and the capillary number $\mathrm{Ca}=\eta U_{l} / \gamma$.

The considered geometry for the numerical simulations is illustrated by a snapshot of the height field shown in Fig. 5.5. We simulated the bridge evolution between two half drops assuming a negligible relative movement of the drops in the early phase. The height field corresponding to the two half drops of radius $R$ is initialised by two parabolas of height $h_{l}=1.0$, which are connected by a sixth-order polynomial at their minimal initial height. The velocity field is initialised as $u \equiv 0$. On the one-dimensional domain
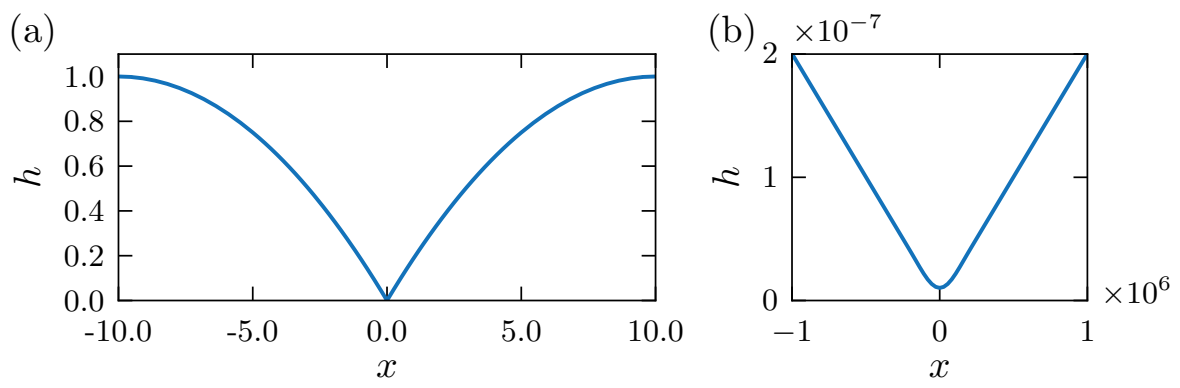

Figure 5.5: (a) Snapshot of an early time $\left(t=2.5 \times 10^{-8}\right.$, viscous case) height profile exhibiting the simulation geometry of the numerical simulations. We impose no flux boundary conditions at both boundaries of the one-dimensional simulation domain. (b) Close-up of the connection between the two drops. 

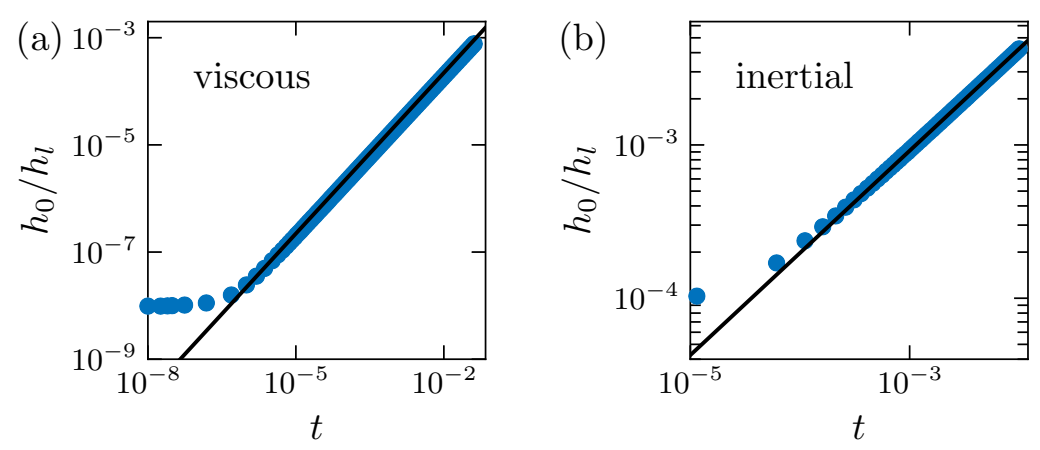

Figure 5.6: Bridge height as obtained from numerical simulations (blue dots) of the time-dependent equations Eqs. 5.12-5.13 for (a) the viscous case (We $=0, \mathrm{Ca}=1)$, and (b) the inertial case (We $=1, \mathrm{Ca}=10^{-4}$ ). The black lines show the evolution of the bridge height as predicted by the similarity solutions of Eqs. 5.4-5.5 and Eqs. 5.4 and 5.8.

$[-R, R]=[-10,10]$ we impose the boundary conditions $h_{x}=h_{x x x}=0$ and $u=0$ at $x=-R, R$. To verify the similarity solutions for the viscous case, we set $\mathrm{We}=0$ and $\mathrm{Ca}=1$. For the inertial case, we consider $\mathrm{We}=1$ and $\mathrm{Ca}=10^{-4}$ (a small viscous contribution is retained for numerical reasons). The simulations are conducted employing a finite element approach using the generic C++ FEM framework OOMPH-LIB [172]. We employ a spatially nonuniform mesh and a second order backward differentiation formula scheme for the time stepping.

The time evolution of the bridge height as obtained for the two aforementioned limiting cases is shown in Fig. 5.6 in comparison to the power law prediction by the corresponding similarity equations Eqs. 5.4-5.5 and Eqs. 5.4 and 5.8. For both cases, the solutions very rapidly approach the similarity solutions in the initial phase of the simulations. The resulting velocity profiles are shown in Fig. 5.2c and Fig. 5.3c.

\subsubsection{Asymmetric thin sheet equation}

For the case of different oil-air and oil-water surface tensions $\left(\gamma \neq \gamma_{\mathrm{ow}}\right)$, and assuming no pressure gradients exist in the vertical direction inside the lens (local equilibrium), a thin sheet equation for the distance between the two interfaces $h=h_{1}+h_{2}$ can be derived which has precisely the same mathematical structure as the symmetric one employed in this chapter. Here, $h_{1}$ and 


\subsection{Appendix}

$h_{2}$ are the distances from the centre of the lens to the oil-air and oil-water interfaces, respectively. The surface tension appearing in this equation is a reduced surface tension $\tilde{\gamma}=\gamma_{\text {ow }} \gamma /\left(\gamma_{\text {ow }}+\gamma\right)$ which is also defined in the context of equilibrium calculations for liquid lenses in de Gennes et al. [69]. As usual in slender theory, the pressure is uniform across the sheet and thereby the capillary pressure jumps must be the same on both interfaces, i.e.,

$$
\gamma_{\text {oa }} \frac{\partial^{2} h_{1}}{\partial x^{2}}=\gamma_{\text {ow }} \frac{\partial^{2} h_{2}}{\partial x^{2}}=\tilde{\gamma} \frac{\partial^{2} h}{\partial x^{2}}
$$

The resulting asymmetric momentum equation is

$$
\rho\left(\frac{\partial u}{\partial t}+u \frac{\partial u}{\partial x}\right)=\frac{4 \eta}{h} \frac{\partial}{\partial x}\left(h \frac{\partial u}{\partial x}\right)+\tilde{\gamma} \frac{\partial^{3} h}{\partial x^{3}} .
$$

In our case, for example for mineral oil, $\gamma_{\text {ow }} \approx 40 \mathrm{mN} / \mathrm{m}$ such that the reduced surface tension becomes $2 \tilde{\gamma} \approx 36.76 \mathrm{mN} / \mathrm{m}$ which is comparable to $\gamma=\gamma_{\text {oa }}=34 \mathrm{mN} / \mathrm{m}$. 



\section{Chapter 6}

\section{Theory for the coalescence of viscous lenses}

Drop coalescence occurs through the rapid growth of a liquid bridge that connects the two drops. At early times after contact, the bridge dynamics are typically self-similar, with details depending on the geometry and viscosity of the liquid. In this paper we analyse the coalescence of two-dimensional viscous drops that float on a quiescent deep pool; such drops are called liquid lenses. The analysis is based on the thin-sheet equations, which were recently shown to accurately capture experiments of liquid lens coalescence. It is found that the bridge dynamics follow a self-similar solution at leading order, but, depending on the large-scale boundary conditions on the drop, significant corrections may arise to this solution. These dynamics are studied in detail using numerical simulations and through matched asymptotics. We show that the liquid lens coalescence can involve a global translation of the drops, a feature that is confirmed experimentally.

@ Published as: Walter Tewes, Michiel A. Hack, Charu Datt, Gunnar G. Peng, and Jacco H. Snoeijer, Theory for the coalescence of viscous lenses, J. Fluid Mech. 928, A11 (2021). 


\subsection{Introduction}

Coalescence of drops is one of the most common capillarity-driven phenomena which can be observed in multiphase fluid dynamics. The early-time dynamics of coalescence are dependent on both the viscosity of the drops and their geometry. Different power laws for the growth of the connecting structure (referred to as "neck" or "bridge") have been found for viscous and inviscid freely suspended drops $[45,57-59,61]$, as well as for sessile drops in the viscous and inviscid limit $[42,44,48,50,158]$. The study of coalescence phenomena is also relevant for many applications where the underlying substrate of the coalescing drops is a liquid. Some examples are wet-on-wet printing [162], emulsions $[165,166]$, and lubricant impregnated substrates $[86,160]$.

Here, we focus on liquid lenses [69], consisting of liquid drops floating on a quiescent pool of another liquid. This case was studied for Newtonian drops [35] and liquid crystals [159], where the authors analysed the growth of the bridge in top-view experiments. In chapter 5 we considered the coalescence of lenses using side-view experiments. This perspective is sketched in Fig. 6.1, providing a quasi-two-dimensional view of the problem. The experiments revealed self-similar dynamics of the bridge profiles, with scaling laws for the bridge height $h_{0}$ with time $t$ that depend on the viscosity of the lenses, see chapter 5. At low viscosity, the dominant balance during coalescence is between surface tension and inertia, and it was found that $h_{0} \sim t^{2 / 3}$. At high viscosity, the dominant balance between surface tension and viscosity leads to $h_{0} \sim t$. These scaling laws are the same as those described in the merging of liquid wedges [56]. Owing to the slender geometry of the drops - typically the contact angle $\theta$ in Fig. 6.1 is small - the coalescence of liquid lenses can be analysed using the thin-sheet equations $[169,173]$. Using a similarity analysis, the experimentally observed inertial and viscous scaling laws are recovered, see chapter 5 . For example, the viscous coalescence speed was found to be $\mathrm{d} \bar{h}_{0} / \mathrm{d} \bar{t} \approx \bar{V}_{0}=0.5525 \gamma \theta^{2} / \eta$, where $\gamma$ and $\eta$ respectively are the drop surface tension and viscosity (for consistency we here use overbars to indicate dimensional variables). This prediction was found to be in very good agreement with experiments.

The viscous similarity analysis, however, contains a salient feature that remains to be explained: the obtained self-similar velocity profile does not decay at large distance from the thin bridge region, but reaches a finite value. This is rather unusual for problems involving coalescence (or drop breakup, c.f., Ref. [171]). Namely, the similarity analysis is typically based on the assumption that the flow remains confined to the scale of the bridge - at large 


\subsection{Introduction}

scale, i.e. the scale of the drop, the flow is usually assumed to vanish. Such is the case for the coalescence of sessile drops as illustrated in Fig. 6.2a. It shows an experimental top-view sequence of merging drops that are in contact with a solid substrate. During the initial growth of the bridge the global features of the drops appear nearly stationary - away from the bridge region one observes only a minute spreading of the drops. Figure $6.2 \mathrm{~b}$ shows the equivalent topview sequence for liquid lenses, for which the situation is manifestly different. Clearly, these floating drops do not remain stationary, but their centres of mass exhibit an inward motion as soon as the drops establish contact. This inward motion is not a small effect. Figure $6.2 \mathrm{c}$ compares the centre of mass velocity $\bar{U}$ (measured in top view) to the bridge coalescence velocity $\bar{V}=\mathrm{d} \bar{h}_{0} / \mathrm{d} \bar{t}$ (measured in side view), showing that the two velocities are proportional. These velocities were obtained using the experimental method described in chapter 5 . We remark that this inward motion does not at all arise for liquid lenses of very low viscosity - this is in line with the inviscid similarity solutions, whose velocity rapidly decays away from the bridge, see chapter 5 .

In this chapter, we provide a detailed analysis of the coalescence of highly viscous lenses and elucidate the coupling between the inner "bridge" solution and the global dynamics of the drops. We treat the problem using the twodimensional thin-sheet equations, reflecting an analysis along the cross-section shown in Fig. 6.1. Such a two-dimensional approximation turned out successful for the geometry of spherical caps, since close to the bridge the length scale in the third dimension, $\mathcal{O}\left(\left(R \bar{h}_{0} / \theta\right)^{1 / 2}\right)$, is much larger than the horizontal and vertical length scales of the bridge, $\mathcal{O}\left(\bar{h}_{0} / \theta\right)$ and $\mathcal{O}\left(\bar{h}_{0}\right)[42,44,176]$.

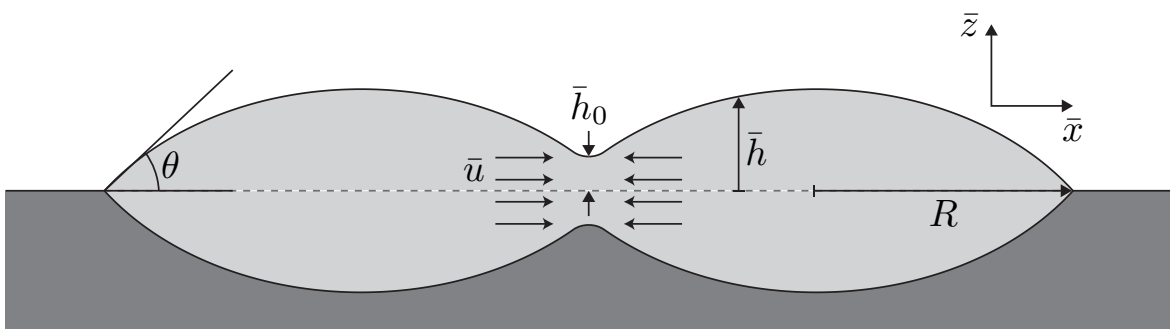

Figure 6.1: Side-view sketch of two coalescing lenses initial radius $R$ and equilibrium contact angle $\theta$. The drop profile is described by $\bar{h}(\bar{x}, \bar{t})$, while the minimal height in the neck region $\bar{h}_{0}(t)=\bar{h}(0, \bar{t})$. The coalescence velocity $\bar{V}$ is defined as $\bar{V}=\mathrm{d} \bar{h}_{0} / \mathrm{d} \bar{t}$. 

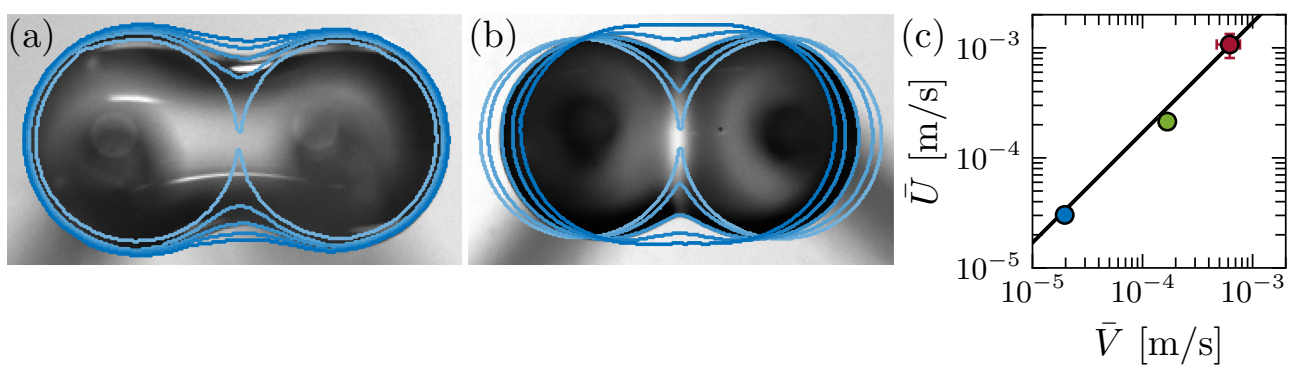

Figure 6.2: Top-view coalescence of drops in the viscous regime. (a) sessile drops in contact with a substrate, (b) liquid lenses floating on a water bath. During lens coalescence we see a clear inward motion of the drops that does not occur for sessile drops. (c) Comparison of the centre of mass velocity $\bar{U}$ and the "bridge" coalescence velocity $\bar{V}=\mathrm{d} \bar{h}_{0} / \mathrm{d} \bar{t}$ for liquid lenses, taken for three oils of different viscosity and nearly identical contact angles (blue: $\eta=115 \mathrm{~Pa} \cdot \mathrm{s}$ and $\theta=27^{\circ}$, green: $\eta=33 \mathrm{~Pa} \cdot \mathrm{s}$ and $\theta=32^{\circ}$, red: $\eta=9 \mathrm{~Pa} \cdot \mathrm{s}$ and $\theta=31^{\circ}$, see chapter 5 for experimental details). The centre of mass velocity $\bar{U}$ is not a small effect, as it is comparable in magnitude to the coalescence velocity $\bar{V}$.

We demonstrate that, in general, the coalescence velocity exhibits significant logarithmic corrections, $\mathcal{O}(1 / \ln t)$, where $t \ll 1$ is the (dimensionless) time after coalescence. On the other hand, the thin-sheet equations admit an outer solution where the drop's centre of mass can migrate freely, closely resembling the motion observed in Fig. 6.2b. In this latter case, the corrections to the leading-order result are much smaller. The analysis is confirmed in detail by comparison to time-dependent numerical simulations of the thin-sheet equations.

The chapter is organised as follows: The governing equations for coalescing lenses are presented in section 6.2. In section 6.3, we study the inner scale dynamics, near the point of coalescence, followed in section 6.4 by an analysis of the outer region, which is where the two different boundary conditions manifest themselves. We end with our conclusion and outlook in section 6.5 . 


\subsection{The viscous thin-sheet equations}

\subsection{The viscous thin-sheet equations}

\subsubsection{Formulation}

Following the approach of chapter 5 , the process of coalescence is modelled by the two-dimensional viscous thin-sheet equations $[169,173]$. The underlying approximations are the following: (i) Similar to sessile drops $[42,44]$ the flow in the bridge region is quasi-two-dimensional in the early stage of coalescence. (ii) The equilibrium contact angle $\theta$ is small, such that a slender body approximation can be employed. (iii) The influence of the bath on the dynamics is negligible, i.e., free slip boundary conditions can be employed at both interfaces of the two-dimensional lenses. (iv) Due to negligible differences in surface tension between the bath and liquid lens and the liquid lens and air, the liquid lenses are assumed to be symmetrical with respect to the bath-air interface - although asymmetric surface tensions can actually be mapped to an "effective" symmetric surface tension (c.f., the appendix of chapter 5). We note that these assumptions are in accordance to the previous experiments of where the ratio of viscosities of the lenses and the bath is more than a thousand and the corresponding surface tension asymmetry is only about ten percent. The former justifies (iii) and the latter (iv).

The resulting model equations for negligible inertia of the flow, in dimensionless variables, take the form

$$
\begin{aligned}
\dot{h}+(h u)^{\prime} & =0, \\
h h^{\prime \prime \prime}+4\left(h u^{\prime}\right)^{\prime} & =0 .
\end{aligned}
$$

Here, $h(x, t)$ and $u(x, t)$, respectively, are the dimensionless interface height and horizontal velocity, and dots and primes indicate derivatives with respect to $t$ and $x$, respectively. Dimensional variables $(\bar{x}, \bar{h}, \bar{u}, \bar{t})$ are scaled as

$$
\bar{x}=R x, \quad \bar{h}=\theta R h, \quad \bar{u}=\frac{\gamma}{\eta} \theta u, \quad \bar{t}=\frac{R \eta}{\theta \gamma} t,
$$

where $R$ is the initial lens radius and $\theta$ is the contact angle (cf. Fig. 6.1). The surface tension is denoted as $\gamma$ and $\eta$ is the viscosity of the liquid inside the lenses. The thin-sheet equations (Eq. 6.1-6.2) correspond to mass and (horizontal) momentum conservation. The latter gives the balance between capillary forces (first term) and viscous forces (second term), while inertia has here been neglected. 
The momentum equation (Eq. 6.2) can be readily integrated to give the horizontal force balance

$$
h h^{\prime \prime}-\frac{1}{2} h^{\prime 2}+4 u^{\prime} h=-\frac{1}{2}+F(t) .
$$

The terms on the left-hand side represent the horizontal force transmitted through the thin drop by pressure, surface tension, and viscous stresses, respectively. We have introduced the constant $-1 / 2$ on the right-hand side corresponding to the total force in the static solution, see Eq. 6.7 below. Therefore $F(t)$ measures any additional horizontal force that arises during the coalescence dynamics.

\subsubsection{Two-dimensional numerical simulations}

In order to illustrate the interplay between the dynamics of the small bridge region and the large bulk of the drops, we simulate the coalescence of twodimensional lenses numerically. Due to symmetry about the point of coalescence $x=0$, we can impose the boundary conditions $h^{\prime}=u=0$ at $x=0$ and focus on the domain $x \geq 0$. We consider two different sets of outer boundary conditions.

The first case corresponds to the experimentally realised setup of two freely floating lenses. In this case, the length $L(t)$ of each lens decreases with time (as can be seen in Fig. 6.2) from its initial value $L(t=0)=2$, and at the edge of the lens we impose the thickness $h(L)$, the contact angle, and conservation of mass

$$
h(L)=0, \quad h^{\prime}(L)=-1, \quad u(L)=\dot{L} .
$$

Substitution into the momentum equation (Eq. 6.4) then yields $F(t) \equiv 0$, i.e., the horizontal force transmitted through the lens is equal to its initial value at all times. With $F=0$ in Eq. 6.4, the condition $h^{\prime}(L)=-1$ is redundant as it follows from $h(L)=0$, so we are left with three boundary conditions $h^{\prime}(0)=u(0)=h(L)=0$ for the third-order governing equations and a fourth condition $\dot{L}=u(L)$ that determines the evolution of $L(t)$.

The second case we consider is one where the lenses do not move, due to a symmetry condition being imposed about the centres of the lenses, such as in a periodic array of simultaneously coalescing lenses,

$$
h^{\prime}(1)=u(1)=0 .
$$




\subsection{The viscous thin-sheet equations}

This yields three boundary conditions $h^{\prime}(0)=u(0)=h^{\prime}(1)=0$ on the governing equations and a fourth condition $u(1)=0$ that determines the unknown $F(t)$.

The key finding is that the different outer boundary conditions will lead to different spatial and temporal dynamics also in the inner region, at the scale of the bridge. As we shall see, the leading-order solution in the two cases remains the same - however, the coalescence velocity can exhibit significant corrections at the next order.

The theoretical initial condition is taken to be the static solution of Eqs. 6.1-6.2 with non-dimensional radius 1 and contact angle 1 (corresponding to the dimensional values $R$ and $\theta$, respectively),

$$
h(x, t=0)=h_{s}(x)=\frac{1}{2} x(2-x),
$$

but in the numerical simulations we add a small-scale perturbation, namely $\left(h_{0 i}-x / 2\right) \exp \left(-x / 2 h_{0 i}\right)$, to initialise the coalescence. The perturbation profile is chosen to satisfy the symmetry boundary condition $h^{\prime}(0)=0$ and have an initial bridge height $h_{0 i}=h_{0}(t=0)$, which is taken to be $10^{-10}$ unless stated otherwise.

We solve the governing equations (Eqs. 6.1-6.4) numerically in Matlab using a finite difference method with a Crank-Nicolson time-stepping scheme. Both the spatial and temporal grids are non-uniform with a relative resolution of $1 \%$, and the discretisation is second-order accurate in both space and time. For the periodic lenses (Eq. 6.6), the computational domain is $0 \leq x \leq 1$. For the free lenses (Eq. 6.5), where the domain $0 \leq x \leq L(t)$ changes with time, we use a rescaled position variable $\hat{x}=2 x / L(t)$ and solve the rescaled equations on the fixed domain $0 \leq \hat{x} \leq 2$ instead. As explained above, four boundary conditions are imposed on the third-order system of equations, in order to determine the additional unknown $\dot{L}(t)$ or $F(t)$.

Some snapshots of height and velocity profiles from the simulations are shown in Fig. 6.3. The free-floating lenses immediately begin to move towards each other at a constant velocity, and eventually merge into one larger lens with double the volume (and hence $\sqrt{2}$ times the radius and height). The periodic lenses instead flatten out towards a uniform height of $1 / 3$, determined by the initial volume in the lens. Importantly, and this is one of the central points of the paper, the velocity profiles are significantly different between the two cases, even at very early times, due to the different outer boundary conditions. We will show how this affects the coalescence velocity, $\dot{h}_{0}$. 
Chapter 6. Theory for the coalescence of viscous lenses
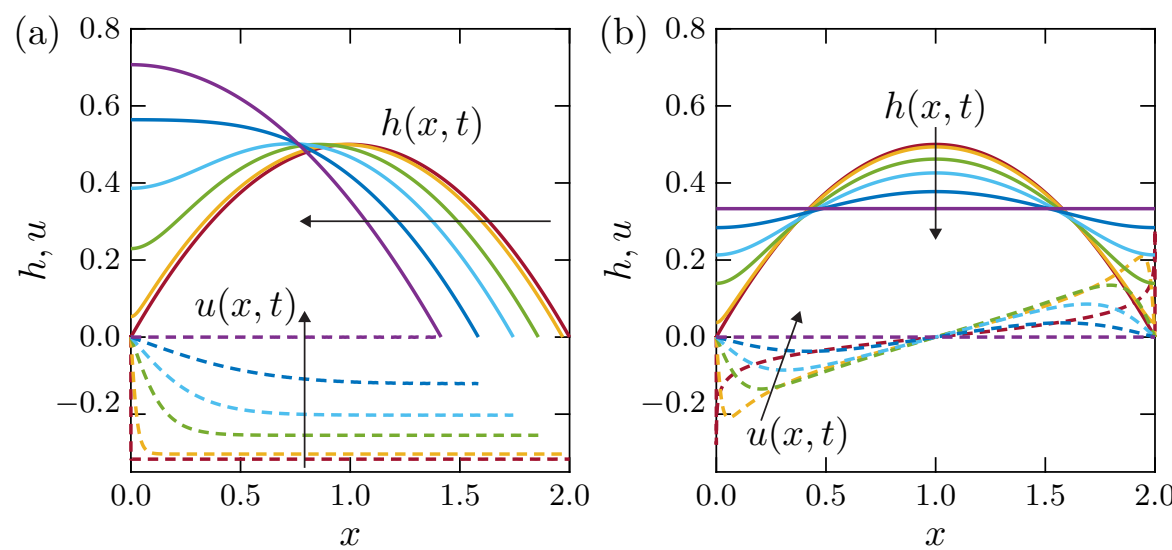

Figure 6.3: Numerical height profiles (solid lines) and velocity profiles (dashed lines) for the coalescence of (a) two free-floating lenses (6.5) and (b) an array of periodic lenses (6.6), evaluated at the times $t=10^{-4}, 0.1,0.5,1,2,10$. The arrows indicate increasing time.

\subsection{The inner region}

In order to study the dynamics of the region close to the point of coalescence, we rescale the variables as

$$
\xi=\frac{x}{h_{0}(t)}, \quad h(x, t)=h_{0}(t) \mathcal{H}(\xi, \tau), \quad u(x, t)=\dot{h}_{0}(t) \mathcal{U}(\xi, \tau),
$$

with $\tau=t$. In choosing the scaling used above, we are motivated by the fact that in the inner region the length scale of importance is the bridge height $h_{0}$. The resulting governing equations become

$$
\begin{aligned}
\frac{h_{0}}{V} \dot{\mathcal{H}}+\mathcal{H}-\xi \mathcal{H}^{\prime}+(\mathcal{H U})^{\prime} & =0, \\
\mathcal{H} \mathcal{H}^{\prime \prime}-\frac{1}{2} \mathcal{H}^{\prime 2}+4 V \mathcal{H} \mathcal{U}^{\prime}+\frac{1}{2}-F(\tau) & =0,
\end{aligned}
$$

where $V=\dot{h}_{0}$ is the unknown coalescence velocity that we wish to determine. At $\xi=0$, the definition $h(0, t)=h_{0}(t)$ and the symmetry about $\xi=0$ yield

$$
\mathcal{H}(0)=1, \quad \mathcal{H}^{\prime}(0)=\mathcal{U}(0)=0 .
$$

The system of Eqs. 6.9-6.10 has three spatial derivatives and accordingly three boundary conditions (Eq. 6.11). However, the equations contain two 


\subsection{The inner region}

unknowns $V$ and $F$ that are determined on matching to the outer solution. The matching necessitates that we evaluate $\mathcal{H}$ and $\mathcal{U}$ as $\xi \rightarrow \infty$, and from Eqs. $6.9-6.10$ we obtain, on neglecting the time-derivative term,

$$
\mathcal{U}=\frac{F}{4 \alpha V} \ln \xi-\beta+\mathcal{O}(\ln \xi / \xi), \quad \mathcal{H}=\alpha \xi+\mathcal{O}(\ln \xi), \quad \text { as } \xi \rightarrow \infty .
$$

These asymptotics contain two degrees of freedom $\alpha$ and $\beta$ that are determined during the solution process. ${ }^{1}$ This matching will be performed explicitly in section 6.4 , but below we already anticipate some of the results necessary to evaluate the inner solutions.

\subsubsection{The leading-order similarity solution}

The leading-order solution to the foregoing equations is a steady self-similar solution, which was previously obtained in chapter 5 . The leading-order quantities $\mathcal{H}_{0}(\xi), \mathcal{U}_{0}(\xi)$ and $V_{0}$ are independent of $\tau$, so that Eqs. 6.9-6.10 reduce to

$$
\begin{aligned}
\mathcal{H}_{0}-\xi \mathcal{H}_{0}^{\prime}+\left(\mathcal{H}_{0} \mathcal{U}_{0}\right)^{\prime} & =0, \\
\mathcal{H}_{0} \mathcal{H}_{0}^{\prime \prime}-\frac{1}{2} \mathcal{H}_{0}^{\prime 2}+4 V_{0} \mathcal{H}_{0} \mathcal{U}_{0}^{\prime}+\frac{1}{2} & =0,
\end{aligned}
$$

where we have anticipated that $F(\tau) \ll 1$ - this indeed is true as we see later. The boundary conditions required to evaluate these quantities are given by Eq. 6.11, complemented by $\mathcal{H}_{0}^{\prime}=1$ as $\xi \rightarrow \infty(\alpha=1)$ set by the leading-order outer solution given in Eq. 6.7. These equations (and also the higher-order equations) are solved numerically in Mathematica using a shooting method on the domain $0 \leq \xi \leq 10^{5}$. We find the leading-order coalescence and far-field translational velocities to be (see chapter 5 )

$$
V_{0}=0.5525, \quad \mathcal{U}_{0} \rightarrow-\beta_{0}=-0.5734, \quad \text { as } \xi \rightarrow \infty .
$$

\footnotetext{
${ }^{1}$ Given that the system is third order in space, there should be a further degree of freedom as $\xi \rightarrow \infty$. However, it can be shown by perturbation analysis of Eqs. 6.9-6.10 (c.f., Ref. [171]), that this degree of freedom appears as a pre-factor of a term decaying through an exponential in $\xi$ as long as $\alpha>0$, which is always the case in this coalescence problem. The degree of freedom does thus not appear in our leading-order asymptotics.
} 


\subsubsection{Next-order corrections}

The solutions $\mathcal{H}_{0}$ and $\mathcal{U}_{0}$ are plotted in Fig. $6.4 \mathrm{a}-\mathrm{b}$, where they are compared with the numerical solutions. The two columns correspond to the two types of boundary conditions, free-floating lenses and periodic lenses. For both cases an excellent agreement is found at early times after coalescence. However, the velocity profiles in Fig. $6.3 \mathrm{a}-\mathrm{b}$ revealed large differences between these two situations. While these differences do not turn up in the leading-order inner

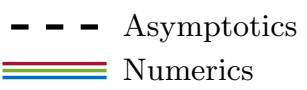

(a)

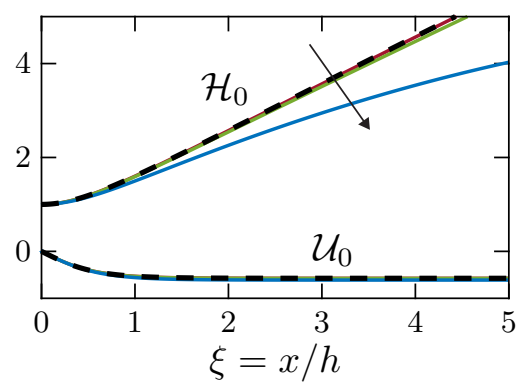

(b)

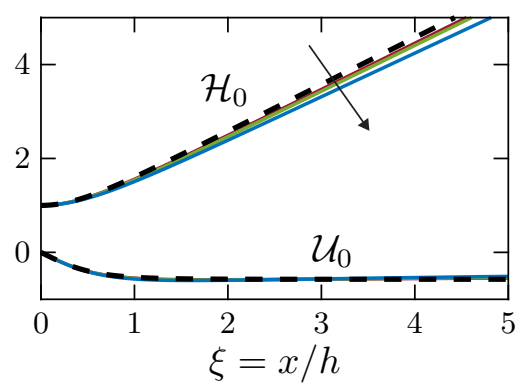

(c)

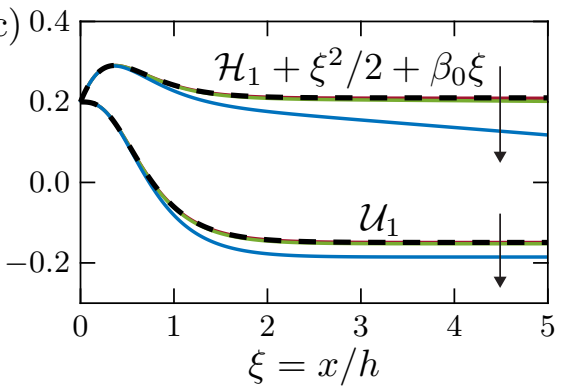

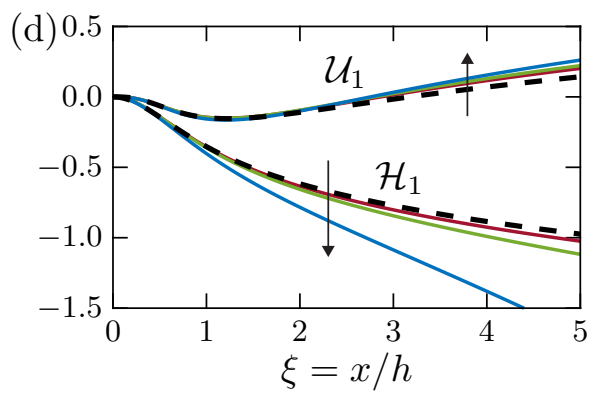

Figure 6.4: Similarity solution profiles -leading order (Eq. 6.15) and first correction (Eqs. 6.20-6.25) - for (a, c) free-floating lenses and (b, d) periodic lenses. Rescaled numerical results are shown for comparison, evaluated at (a, c) $t=10^{-3}, 10^{-2}, 10^{-1}$ and (b, d) $t=10^{-4}, 10^{-3}, 10^{-2}$. The numerical height profile is transformed as $\mathcal{H}_{0, \text { num }}=h / h_{0}$ and $\mathcal{H}_{1, \text { num }}=\left(h / h_{0}-\mathcal{H}_{0, \text { asy }}\right) / \epsilon$ where $\epsilon=h_{0}$ for free lenses and $\epsilon=F$ for periodic lenses, and the velocity is transformed similarly. In order to resolve the $\mathcal{O}\left(10^{-3}\right)$ corrections in $(\mathrm{c})$, the numerical simulation was performed with a resolution of $0.1 \%$, which restricted $h_{0 i}$ to the larger value $10^{-6}$. The arrows indicate increasing time. 


\subsection{The inner region}

solutions $\mathcal{H}_{0}$ and $\mathcal{U}_{0}$, they impact the next-order corrections. As we will see, this also has a strong effect on the evolution of the coalescence velocity $V$. We therefore now address the inner solution beyond the leading order.

\subsubsection{The case $F(\tau) \equiv 0$}

In the case of free-floating lenses, for which $F(\tau) \equiv 0$, it turns out that we can set up a consistent expansion based on the expansion parameter $h_{0}(\tau)$. On substituting the expansions

$$
\begin{aligned}
\{\mathcal{H}(\tau), \mathcal{U}(\tau), V(\tau), \beta(\tau)\}=\left\{\mathcal{H}_{0}, \mathcal{U}_{0}, V_{0}, \beta_{0}\right\} & \\
& +h_{0}(\tau)\left\{\mathcal{H}_{1}, \mathcal{U}_{1}, V_{1}, \beta_{1}\right\}+\mathcal{O}\left(h_{0}^{2}\right)
\end{aligned}
$$

(where the dependence of $\mathcal{H}$ and $\mathcal{U}$ on $\xi$ is understood) into Eqs. 6.9-6.11 we obtain, at $\mathcal{O}\left(h_{0}\right)$,

$$
\begin{aligned}
2 \mathcal{H}_{1}-\xi \mathcal{H}_{1}^{\prime}+\left(\mathcal{H}_{0} \mathcal{U}_{1}+\mathcal{H}_{1} \mathcal{U}_{0}\right)^{\prime} & =0 \\
\mathcal{H}_{0} \mathcal{H}_{1}^{\prime \prime}+\mathcal{H}_{1} \mathcal{H}_{0}^{\prime \prime}-\mathcal{H}_{0}^{\prime} \mathcal{H}_{1}^{\prime}+4\left(V_{0} \mathcal{H}_{0} \mathcal{U}_{1}^{\prime}+V_{0} \mathcal{H}_{1} \mathcal{U}_{0}^{\prime}+V_{1} \mathcal{H}_{0} \mathcal{U}_{0}^{\prime}\right) & =0 \\
\mathcal{H}_{1}(0)=\mathcal{H}_{1}^{\prime}(0)=\mathcal{U}_{1}(0) & =0
\end{aligned}
$$

The differential equations for $\mathcal{H}_{1}$ and $\mathcal{U}_{1}$ are of third order with three boundary conditions, so one further condition is required in order to determine the unknown $V_{1}$. This is obtained from a matching with the outer region. As $\xi \rightarrow \infty$, the generic solutions to Eqs. 6.17-6.19 are quadratic, $\mathcal{H}_{1} \propto \xi^{2}$, and so will need to match the second derivative $h_{s}^{\prime \prime}(0)=-1\left(\right.$ an $\mathcal{O}\left(h_{0}\right)$ quantity when expressed in inner variables) of the outer solution. We thus impose $\mathcal{H}_{1}^{\prime \prime} \rightarrow-1$ as $\xi \rightarrow \infty$, and obtain the numerical solutions plotted in Fig. 6.4c, with the coefficients

$$
V_{1}=-0.7625, \quad \mathcal{U}_{1} \rightarrow-\beta_{1}=-0.3492, \quad \text { as } \xi \rightarrow \infty .
$$

\subsubsection{The case of non-zero $F(\tau)$}

When the horizontal force $F(\tau)$ is non-zero, the next-order corrections are at $\mathcal{O}(F)$. As we shall see in the next section, these corrections dominate the $\mathcal{O}\left(h_{0}\right)$ corrections that arise due to time evolution of the outer solution, since $F$, though unknown yet, is evaluated to be $\mathcal{O}\left(1 / \ln \left(h_{0}\right)\right)$. For this reason, we now expand the solution in terms of $F$, i.e.

$$
\begin{aligned}
\{\mathcal{H}(\tau), \mathcal{U}(\tau), V(\tau), \beta(\tau)\}=\left\{\mathcal{H}_{0}, \mathcal{U}_{0}, V_{0}, \beta_{0}\right\} & \\
& +F(\tau)\left\{\mathcal{H}_{1}, \mathcal{U}_{1}, V_{1}, \beta_{1}\right\}+\mathcal{O}\left(F^{2}\right) .
\end{aligned}
$$


After substituting into the governing equations, we obtain, at $\mathcal{O}(F)$,

$$
\begin{aligned}
\mathcal{H}_{1}-\xi \mathcal{H}_{1}^{\prime}+\left(\mathcal{H}_{0} \mathcal{U}_{1}+\mathcal{H}_{1} \mathcal{U}_{0}\right)^{\prime} & =0, \\
\mathcal{H}_{0} \mathcal{H}_{1}^{\prime \prime}+\mathcal{H}_{1} \mathcal{H}_{0}^{\prime \prime}-\mathcal{H}_{0}^{\prime} \mathcal{H}_{1}^{\prime}+4\left(V_{0} \mathcal{H}_{0} \mathcal{U}_{1}^{\prime}+V_{0} \mathcal{H}_{1} \mathcal{U}_{0}^{\prime}+V_{1} \mathcal{H}_{0} \mathcal{U}_{0}^{\prime}\right) & =1, \\
\mathcal{H}_{1}^{\prime}(0)=\mathcal{U}_{1}(0)=\mathcal{H}_{1}(0) & =0 .
\end{aligned}
$$

Since the corrections to the outer solution at $\mathcal{O}\left(h_{0}\right)$ are to be neglected when matching the inner and outer solutions at $\mathcal{O}(F)$, we now impose the condition $\mathcal{H}_{1}^{\prime} \rightarrow 0$ as $\xi \rightarrow \infty$. On solving these equations numerically, we obtain the solution profiles plotted in Fig. $6.4 \mathrm{~d}$ and the coefficients

$$
V_{1}=-0.6227, \quad \beta_{1}=0.7079 .
$$

As $F=\mathcal{O}\left(1 / \ln h_{0}\right)$ will typically not be very small, it is useful to calculate the second-order $\mathcal{O}\left(F^{2}\right)$ correction, which includes contributions proportional to $F^{2}$ as well as contributions proportional to $\left(h_{0} / \dot{h}_{0}\right) \dot{F}$. We perform this calculation in appendix 6.6.1.

\subsection{The outer region and matching}

We now turn to the outer solution, and calculate the corrections to the initial static profile $h_{s}$ (Eq. 6.7) that are generated by the coalescence in the bridge region. Time integration of the evolution Eq. 6.1 from this initial condition yields

$$
h(x, t)=h_{s}(x)-\int_{0}^{t}(u(x, \hat{t}) h(x, \hat{t}))^{\prime} \mathrm{d} \hat{t} .
$$

The outer solution, where $h, u, x=\mathcal{O}(1)$, is thus given by the initial profile $h_{s}(x)$ with an $\mathcal{O}(t)=\mathcal{O}\left(h_{0}\right)$ correction. Integration of the momentum (Eq. 6.4) using $h=h_{s}+\mathcal{O}\left(h_{0}\right)$ then yields the outer-region velocity profile

$$
u_{o}=\frac{F(t)}{4} \ln \frac{x}{2-x}+B(t)+\mathcal{O}\left(h_{0}\right) .
$$

The coefficients $B(t)$ and $F(t)$ are determined by boundary conditions and by matching to the inner solution.

\subsubsection{The case of freely floating lenses}

For coalescing free lenses, the boundary conditions (Eq. 6.5) result in no additional horizontal force, $F(t) \equiv 0$, and hence the leading-order velocity profile (Eq. 6.27) is a uniform translation. The translation velocity is given by 


\subsection{The outer region and matching}

the far-field behaviour (Eq. 6.15) of the inner solution, so at leading order $u_{o} \approx-V_{0} \beta_{0}=-0.3168$, which is independent of time.

The first corrections in the outer solution then come in at $\mathcal{O}(t)=\mathcal{O}\left(h_{0}\right)$. Time integration of the mass conservation equation (6.1) yields the result

$$
h(x, t)=h_{s}(x)-t u_{0} h_{s}^{\prime}(x)+\mathcal{O}\left(h_{0}^{2}\right)=h_{s}\left(x-u_{0} t\right)+\mathcal{O}\left(h_{0}^{2}\right),
$$

which reveals that the first-order correction simply represents a translation of the initial profile $h_{s}(x)$ by the steady leading-order velocity $u_{o}<0$. In fact, it can be shown that to all orders in $h_{0}$, the velocity profile is spatially uniform and hence the drop is undergoing pure translation, with deformation only occurring in the bridge region $x=\mathcal{O}\left(h_{0}\right)$.

In order to match with the bridge region, we substitute $x=h_{0} \xi$ into Eq. 6.28 and expand in powers of $h_{0}$, making use of $t u_{o} \approx-V_{0} \beta_{0} t \approx-\beta_{0} h_{0}$. This yields

$$
h=h_{0}\left[\left(\xi-h_{0} \frac{\xi^{2}}{2}\right)+\beta_{0}\left(1-h_{0} \xi\right)+\ldots\right],
$$

Comparing this with the definition of the inner variables (Eq. 6.8), we now identify the appropriate far-field behaviour of the inner solution to be

$$
\mathcal{H}_{1}(\xi) \sim-\frac{\xi^{2}}{2}-\beta_{0} \xi+\ldots, \quad \text { as } \xi \rightarrow \infty .
$$

This yields the condition $\mathcal{H}_{1}^{\prime \prime} \rightarrow-1$ as $\xi \rightarrow \infty$ that we anticipated in section 6.3.2.1, which was imposed to obtain the solution Eq. 6.20.

The resulting expression for the coalescence velocity is, from Eq. 6.15 and Eq. 6.20,

$$
\dot{h}_{0}(t)=0.5525-0.7625 h_{0}+\mathcal{O}\left(h_{0}^{2}\right),
$$

predicting a linear correction to the coalescence velocity. This prediction is tested quantitatively in comparison with numerical simulations in Fig. 6.5. The result (Eq. 6.31) is shown as a dashed line labelled "free", while the solid lines are numerics that were initialised at three different initial bridge heights $h_{0 i}$. After a short time, the numerical curves for free lenses rapidly converge to the predicted asymptotics (Eq. 6.31). Note that in typical experimental conditions where $h_{0} \sim 10^{-3} \cdots 10^{-2}$, the coalescence velocity is very close to the leading-order value $V_{0}$. 


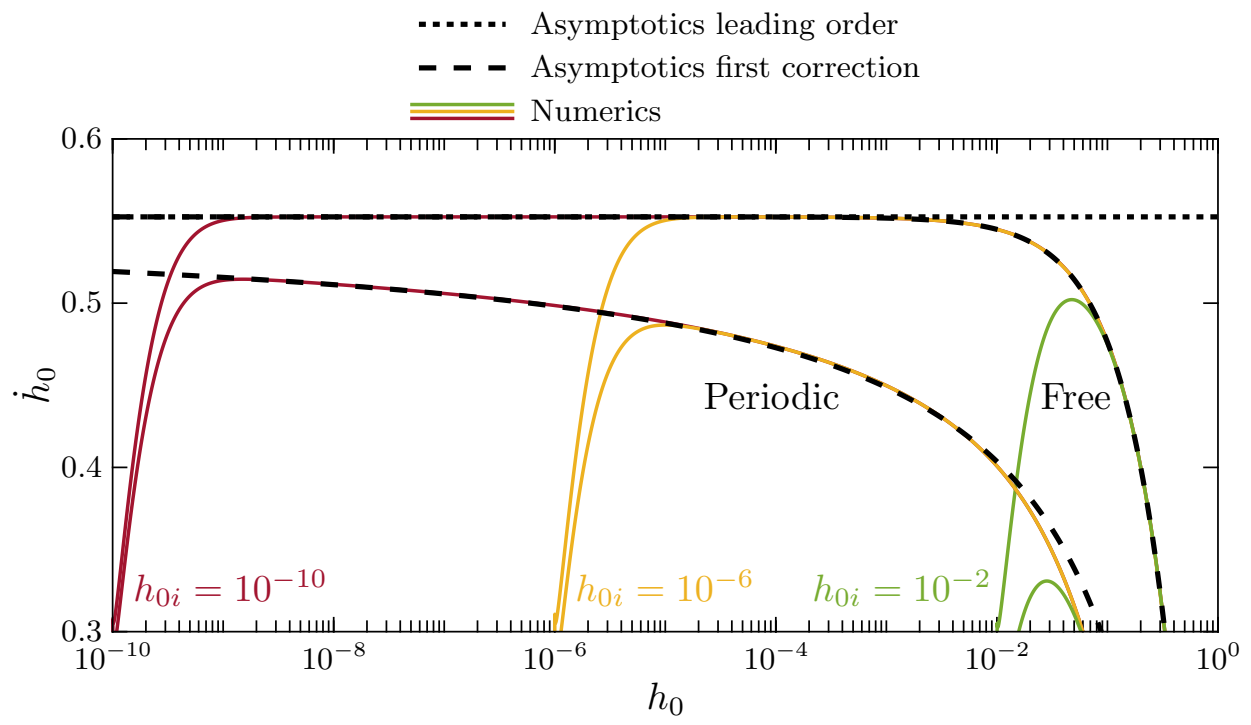

Figure 6.5: The dependence of the coalescence velocity $V=\dot{h}_{0}$ on the bridge height $h_{0}$, for both free-floating (Eq. 6.5) and periodic (Eq. 6.6) lenses. Numerical results for three different values of the initial bridge height $h_{0 i}$ are shown (solid lines). The asymptotic results for the free-floating and periodic lenses are given (dashed lines), respectively, by Eq. 6.31 and Eq. 6.35.

\subsubsection{The case of periodic lenses}

The periodic lenses come with the symmetry boundary condition (Eq. 6.6) on the velocity profile (Eq. 6.27). This yields $B(t) \equiv 0$ and hence

$$
u_{o}=\frac{F(t)}{4} \ln \frac{x}{2-x} .
$$

This logarithmic velocity at small $x$ can indeed be matched to the velocity at large $\xi$ from the inner region calculated in section 6.3.2.2. Specifically, we equate Eq. 6.32 (taking the limit $x \ll 1$ ) with the far-field inner velocity profile Eq. 6.12 (using $u=V \mathcal{U}$ and $\xi=x / h_{0}$ ),

$$
\frac{F(t)}{4} \ln \frac{x}{2}=\frac{F(t)}{4} \ln \frac{x}{h_{0}}-V \beta,
$$

where it is noted that $V$ and $\beta$ are expanded in $F$ themselves. 


\subsection{Conclusion}

Equation 6.33 finally enables us to express $F$ in terms of $h_{0}$, which to leading order gives the result

$$
F=\frac{4 V \beta}{\ln \left(2 / h_{0}\right)}=\frac{4 V_{0} \beta_{0}}{\ln \left(2 / h_{0}\right)}+\mathcal{O}\left(\ln \left(2 / h_{0}\right)^{-2}\right) .
$$

This confirms the slow, logarithmic decay of the force term, suggesting strong corrections with respect to the leading-order similarity solution. Most importantly, this leads us to the sought-after coalescence velocity for periodic drops

$$
\dot{h}_{0}=V_{0}+V_{1} F+\mathcal{O}\left(F^{2}\right)=V_{0}+\frac{\hat{V}_{1}}{\ln \left(2 / h_{0}\right)}+\mathcal{O}\left(\ln \left(2 / h_{0}\right)^{-2}\right),
$$

where $V_{0}=0.5525, V_{1}=-0.6227$ and $\hat{V}_{1}=4 V_{0} \beta_{0} V_{1}=0.7892$. The correction to the coalescence velocity $\left(\mathcal{O}\left(\ln \left(2 / h_{0}\right)^{-2}\right)\right)$ is calculated in appendix 6.6.1.

Once again, the asymptotic prediction is tested quantitatively in comparison with numerical simulations in Fig. 6.5. The result Eq. 6.35 is shown as a dashed line labelled "periodic", while the solid lines are numerics that were initialised at three different initial heights $h_{0 i}$; again the numerics are in excellent agreement with the asymptotics. We notice a dramatic difference between the coalescence velocities for periodic drops as compared to the free drops, owing to the logarithmic decay of $F$. At values where $h_{0} \sim 10^{-3} \cdots 10^{-2}$, the coalescence velocity for periodic drops is significantly below the leading-order value $V_{0}$.

\subsection{Conclusion}

In this chapter, we have analysed the coalescence dynamics of viscous liquid lenses using both matched asymptotics and numerical simulations. We restricted our attention to the case where the flow in the bath is negligible, which is a consistent approximation for lenses of high viscosity, and where the contact angles are small to enable a slender thin-sheet description. In addition, following a previously used assumption in coalescence, we treated the problem as quasi-two-dimensional. The common scenario in coalescence and pinch-off, is that the flow remains localised into the narrow bridge region, while the far field remains stationary. Here, we have found that this is not the case for viscous lens coalescence, and demonstrated that the bridge region affects the global dynamics, using both matched asymptotics and numerical simulations. 
For freely floating two-dimensional viscous lenses, as soon as the lenses start coalescing there is a motion of the outer contact line towards the point of coalescence. In dimensional form, the growth rate of the bridge height $\bar{h}_{0}(\bar{t})=\bar{h}(\bar{x}=0, \bar{t})$ (Eq. 6.31), and the horizontal translation velocity of the drop's centre of mass $\bar{u}_{o}$ (Eqs. 6.15 and 6.20) can be written as

$$
\begin{aligned}
& \bar{V}=\dot{\bar{h}}_{0}=\left(0.5525+0.7625 \frac{\bar{h}_{s}^{\prime \prime}(0) \bar{h}_{0}}{\theta^{2}}+\ldots\right) \frac{\gamma \theta^{2}}{\eta}, \\
& \bar{U}=\bar{u}_{o}=-\left(0.3168-0.2443 \frac{\bar{h}_{s}^{\prime \prime}(0) \bar{h}_{0}}{\theta^{2}}+\ldots\right) \frac{\gamma \theta}{\eta} .
\end{aligned}
$$

Here, we have eliminated the lens radius $R$ in favour of the second derivative $\bar{h}_{s}^{\prime \prime}(0)=-\theta / R$ of the initial condition Eq. 6.7 , in order to highlight that the coalescence process depends only on the local shape of the lens near the bridge.

For a periodic array of two-dimensional viscous lenses, the centres of mass of the drops do not move due to symmetry. Instead, a horizontal force is generated that resists the inward motion towards the bridge, which results in a logarithmic velocity profile in the lens. In dimensional form, the growth rate of the bridge Eq. 6.35 and the additional force generated Eq. 6.34 are then given by

$$
\begin{aligned}
& \bar{V}=\dot{\bar{h}}_{0}=\left(0.5525+\frac{0.7892}{\ln \left(2 R \theta / \bar{h}_{0}\right)}+\ldots\right) \frac{\gamma \theta^{2}}{\eta}, \\
& \bar{F}=\left(\frac{1.267}{\ln \left(2 R \theta / \bar{h}_{0}\right)}+\ldots\right) \gamma \theta^{2} .
\end{aligned}
$$

A further, second-order, correction is calculated in appendix 6.6.1. It is important to note that the corrections are logarithmic in time, so these can be significant even at the very early stages of coalescence. In fact, logarithmic corrections also arise for viscous coalescence of freely suspended drops $[57,177]$. The structure of the problem is, however, different. In the freely suspended case the neck grows as $h \sim t \ln t$, which in contrast to Eq. 6.38 does not exhibit a finite velocity at early times.

It is of interest to compare these two-dimensional, slender predictions with the experiments shown in Fig. 6.2c. In chapter 5 it was already shown that the experimental vertical coalescence velocity $\bar{V}$ was in very good quantitative agreement with the leading-order prediction of Eqs. 6.36-6.37. Indeed, for freely floating drops, the higher-order terms are expected to be negligible in the experimental range. The current theory predicts a ratio of horizontal 


\subsection{Conclusion}

to vertical velocity $\bar{U} / \bar{V} \approx 0.57 / \theta$ for the freely moving drops, which for the experimental contact angles amounts to $\bar{U} / \bar{V} \approx 1.1$. The experimentally measured ratio in Fig. 6.2 c was found $\bar{U} / \bar{V} \approx 1.7$, which implies an even stronger centre of mass motion than predicted. This quantitative disparity could be due to three-dimensional effects, or due to the fact that the contact angles are not very small. Still, our theory offers an explanation for the appearance of a centre of mass motion for viscous lenses, and provides the relevant scaling laws. This centre of mass motion is not observed for inertial drops, which is in accordance with the decaying velocity in the inertial similarity solution presented in chapter 5. For future experiments, it might be of interest to study coalescence while the centres of the drop are prevented from translating towards each other (e.g. by attaching the drops to fixed capillaries). In that case, we expect the appearance of significant (logarithmic) corrections to the coalescence velocity.

Besides three-dimensional effects, it is also of interest to discuss the influence of gravity. Gravity becomes important if the (dimensional) radius $R$ of the lenses is no longer small compared with the capillary length $\ell_{c}=\sqrt{\gamma / \Delta \rho g}$, where $\Delta \rho$ is the difference in density between the lenses and either of the external fluids. In this case, we expect gravity to flatten the static lens profile $h_{s}(x)$ (Eq. 6.7), but near the rim of the lens, when $x \ll \ell_{c}$, gravity becomes negligible and the capillary thin-sheet equations (Eqs. 6.1-6.2) and contact angle $\theta$ are recovered, and hence the leading-order inner solution Eq. 6.15 will still hold. For (two-dimensional) free lenses, which can undergo uniform translation even in the presence of gravity, the $\mathcal{O}\left(h_{0}\right)$ correction Eqs. 6.36-6.37 also holds, but with a modified value of $h_{s}^{\prime \prime}(0)$ that simply follows from the static drop. For periodic lenses, the correction becomes more involved as the outer velocity profile Eq. 6.27 is affected by the change in $h_{s}$.

More generally, the dynamical structure of the problem bears a strong similarity with drop spreading on a rigid substrate, where the motion of the contact line also induces a weak flow on the scale of the drop [113]. The spreading velocity exhibits a logarithmic dependence on the scale separation between drop size and the characteristic scale of the contact line in that case too. An important difference, however, is that for drop spreading the universal leadingorder similarity solution for the inner problem captures the phenomenon, as it has only algebraically small corrections. Here, we found that for viscous lens coalescence, the corrections are themselves only logarithmically small, and therefore can be significant. 
Chapter 6. Theory for the coalescence of viscous lenses

\subsection{Appendix}

\subsubsection{Details of the inner solution for non-zero $F$}

We calculate both the $\mathcal{O}(F)$ and $\mathcal{O}\left(F^{2}\right)$ corrections to the leading-order result Eq. 6.15 by expanding

$$
\mathcal{H}=\mathcal{H}_{0}+F \mathcal{H}_{1}+F^{2} \mathcal{H}_{2}+\frac{h_{0}}{\dot{h}_{0}} \dot{F} \mathcal{H}_{T}+\mathcal{O}\left(F^{3}\right)
$$

and similarly for $\mathcal{U}, V$ and $\beta$. Note that, since we anticipate that $F$ is $\mathcal{O}(1 / \ln t)$, the terms with subscript 2 and $T$ are both $\mathcal{O}\left(F^{2}\right)$.

We substitute the expansion into the equations Eqs. 6.9-6.11, together with the matching condition $\mathcal{H}^{\prime}(\infty)=1$ which is accurate to all orders in $F$, and identify coefficients. The resulting governing equations for the corrections $\mathcal{H}_{\Delta}, \mathcal{U}_{\Delta}$ and $V_{\Delta}$, where $\Delta=1,2, T$, are given by

$$
\begin{aligned}
& \left(\begin{array}{c}
\mathcal{H}_{\Delta}-\xi \mathcal{H}_{\Delta}^{\prime}+\left(\mathcal{H}_{0} \mathcal{U}_{\Delta}+\mathcal{H}_{\Delta} \mathcal{U}_{0}\right)^{\prime} \\
\mathcal{H}_{0} \mathcal{H}_{\Delta}^{\prime \prime}+\mathcal{H}_{\Delta} \mathcal{H}_{0}^{\prime \prime}-\mathcal{H}_{0}^{\prime} \mathcal{H}_{\Delta}^{\prime}+4\left(V_{0} \mathcal{H}_{0} \mathcal{U}_{\Delta}^{\prime}+V_{0} \mathcal{H}_{\Delta} \mathcal{U}_{0}^{\prime}+V_{\Delta} \mathcal{H}_{0} \mathcal{U}_{0}^{\prime}\right)
\end{array}\right)= \\
& \underbrace{\left(\begin{array}{l}
0 \\
1
\end{array}\right)}_{\Delta=1}, \underbrace{\left(\begin{array}{c}
-\left(\mathcal{H}_{1} \mathcal{U}_{1}\right)^{\prime} \\
-\mathcal{H}_{1} \mathcal{H}_{1}^{\prime \prime}+\frac{1}{2} \mathcal{H}_{1}^{\prime 2}-4\left(V_{0} \mathcal{H}_{1} \mathcal{U}_{1}^{\prime}+V_{1} \mathcal{H}_{0} \mathcal{U}_{1}^{\prime}+V_{1} \mathcal{H}_{1} \mathcal{U}_{0}^{\prime}\right)
\end{array}\right)}_{\Delta=2}, \underbrace{\left(\begin{array}{c}
-\mathcal{H}_{1} \\
0
\end{array}\right)}_{\Delta=T},
\end{aligned}
$$

and the boundary conditions are $\mathcal{H}_{\Delta}^{\prime}(0)=\mathcal{U}_{\Delta}(0)=\mathcal{H}_{\Delta}(0)=\mathcal{H}_{\Delta}^{\prime}(\infty)=0$.

Solving these equations numerically using Mathematica yields the results

$$
\begin{aligned}
& V_{0}=0.5525, \quad V_{1}=-0.6227, \quad V_{2}=-0.1267, \quad V_{T}=0.3028, \\
& \beta_{0}=0.5734, \quad \beta_{1}=0.7079, \quad \beta_{2}=0.8728, \quad \beta_{T}=-0.5277 \text {, }
\end{aligned}
$$

for the coefficients in the far-field behaviour Eq. 6.12.

We can then use the matching (Eq. 6.34) to obtain the results

$$
\begin{aligned}
F & =\frac{4\left(V_{0}+V_{1} F+\mathcal{O}\left(F^{2}\right)\right)\left(\beta_{0}+\beta_{1} F+\mathcal{O}\left(F^{2}\right)\right)}{\ln \left(2 / h_{0}\right)} \\
& =\frac{4 V_{0} \beta_{0}}{\ln \left(2 / h_{0}\right)}+\frac{16 V_{0} \beta_{0}\left(V_{0} \beta_{1}+V_{1} \beta_{0}\right)}{\ln \left(2 / h_{0}\right)^{2}}+\mathcal{O}\left(\ln \left(2 / h_{0}\right)^{-3}\right),
\end{aligned}
$$




\subsection{Appendix}

and

$$
\begin{aligned}
\dot{h}_{0} & =V_{0}+V_{1} F+V_{2} F^{2}+V_{T}\left(h_{0} / \dot{h}_{0}\right) \dot{F}+\mathcal{O}\left(F^{3}\right), \\
& =V_{0}+\frac{\hat{V}_{1}}{\ln \left(2 / h_{0}\right)}+\frac{\hat{V}_{2}}{\ln \left(2 / h_{0}\right)^{2}}+\mathcal{O}\left(\ln \left(2 / h_{0}\right)^{-3}\right), \\
& =V_{0}+\frac{\hat{V}_{1}}{\ln \left(c / h_{0}\right)}+\mathcal{O}\left(\ln \left(2 / h_{0}\right)^{-3}\right),
\end{aligned}
$$

where $V_{0}=0.5525, \hat{V}_{1}=4 V_{0} \beta_{0} V_{1}=0.7892$, and

$$
\begin{aligned}
\hat{V}_{2} & =16 V_{0}^{2} \beta_{0}^{2} V_{2}+16 V_{0} \beta_{0}\left(V_{0} \beta_{1}+V_{1} \beta_{0}\right) V_{1}+4 V_{0} \beta_{0} V_{T}=0.07272, \\
c & =2 \exp \left(-\hat{V}_{2} / \hat{V}_{1}\right)=2.19 .
\end{aligned}
$$

The second-order coefficient $\hat{V}_{2}$ is coincidentally quite small when $\dot{h}_{0}$ is expanded in terms of $\ln \left(2 / h_{0}\right)$, so including the second-order correction in Fig. 6.5 would not have a noticeable effect. However, when $\dot{h}_{0}$ is expanded in terms of, e.g., $\ln \left(1 / h_{0}\right)$ or $\ln (1 / t)$, the second-order correction yields a significant improvement. 



\section{Chapter 7}

\section{When elasticity affects drop coalescence}

The breakup and coalescence of drops are elementary topological transitions in interfacial flows. The breakup of a drop changes dramatically when polymers are added to the fluid. With the strong elongation of the polymers during the process, long threads connecting the two drops appear prior to their eventual pinch-off. Here, we demonstrate how elasticity affects drop coalescence, the complement of the much studied drop pinch-off. We reveal the emergence of an elastic singularity, characterised by a diverging interface curvature at the point of coalescence. Intriguingly, while the polymers dictate the spatial features of coalescence, they hardly affect the temporal evolution of the bridge. These results are explained using a novel viscoelastic similarity analysis and are relevant for drops created in bio-fluids, coating sprays and inkjet printing.

@ Submitted as: Pim J. Dekker, Michiel A. Hack, Walter Tewes, Charu Datt, Ambre Bouillant, Jacco H. Snoeijer, When elasticity affects drop coalescence. 


\subsection{Introduction}

Viscoelastic liquids are materials that can flow like ordinary liquids, yet respond elastically when excited by rapid deformations [178]. A prime example is provided by a ball of silly putty-it bounces like a rubber ball, but spreads out like a viscous liquid when left at rest on a table. Viscoelastic liquids are ubiquitous in bio-fluids and in technologies such as coating, printing, and polymer processing, and their flow poses many challenges. Of particular interest is how viscoelastic liquids behave near singularities, such as flows around sharp edges, bubble cusps, or during the breakup of drops [39,179-191]. These flows involve regions of extreme polymer stretching, which is why, for example, fluids containing polymers can produce long and stable threads during drop breakup (see Fig. 7.1a) [188]. Liquid threads are indeed observed for bio-fluids such as saliva, where they play a role in the generation of aerosols [192].

In contrast to breakup (e.g., Refs. [36,193] and references therein), few studies exist on the coalescence dynamics of viscoelastic drops (see Fig. 7.1bc) [194]. Previous works exploring the effects of viscoelasticity on drop coalescence have focussed on film drainage as the two drops come together before coalescence $[195,196]$. Here our focus is on the merging after initial contact between drops, which is mediated by the growth of a bridge that typ-

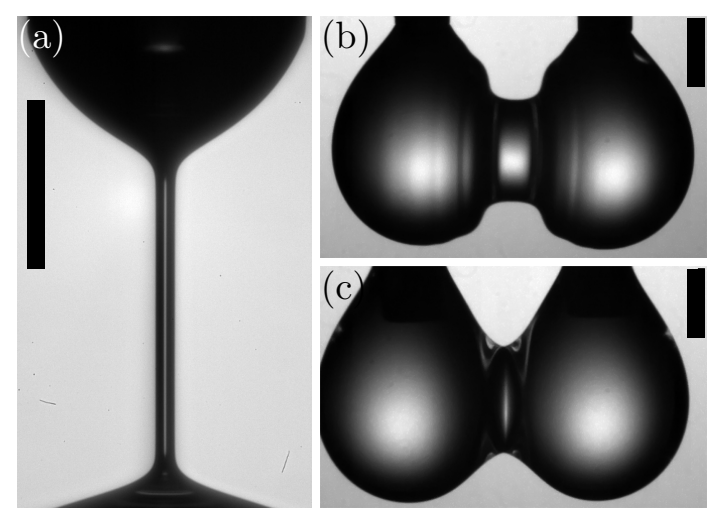

Figure 7.1: Pinching and merging of viscoelastic polymer solutions. (a) Pinch-off of a $2.0 \mathrm{wt} \%$ PEO drop gives rise to elongated threads. Coalescence of (b) pure water drops, and (c) $2.0 \mathrm{wt} \%$ PEO drops. Polymer stretching inside the bridge markedly enhances the curvature of the connecting bridge. The scale bars indicate $500 \mu \mathrm{m}$. 


\subsection{Experimental methods}

ically grows as $h \sim t^{\alpha}$ where $h$ is the bridge size and $t$ is the time after contact $[42,44,45,50,54,57,58,60,177,197-199]$. The corresponding rate-ofdeformation is estimated to diverge as $h / h \sim 1 / t$, and one thus anticipates a strongly elastic response during the initial phase of coalescence, when the flow time scale is much shorter than the relaxation time of polymers in the fluid - much like in the case of bouncing putty. It has thus far remained elusive how polymers and the singularity at the moment of contact interact, and how this affects the merging process.

In this chapter, we resolve the coalescence dynamics of viscoelastic liquids by experiments on aqueous polymer solutions of varying concentration. We consider the coalescence of both freely suspended drops and of drops in contact with a substrate, c.f., Fig. 7.2. For both cases it is found that polymer stress dramatically changes the spatial structure of the bridge, as evident in Figs. 7.1b-c. Yet, surprisingly, the temporal growth of the bridge is only mildly affected by the polymers. These features are explained and quantified by a similarity theory for sessile drops, revealing that viscoelastic coalescence is very different in nature compared to pinch-off.

\subsection{Experimental methods}

The coalescence experiments were performed using viscoelastic solutions of polyethylene oxide (PEO, $M_{W}=4.0 \times 10^{6} \mathrm{~g} / \mathrm{mol}$, Sigma-Aldrich) in water (MilliQ, Millipore Corporation). Each solution was mixed with a magnetic stirrer for at least 24 hours, resulting in highly homogeneous solutions. While drop breakup is modified already for minute addition of polymer (c.f., Refs. [39, 191]), elastic effects in coalescence require very high concentrations - up to 1.0 wt\% and $2.0 \mathrm{wt} \%$. We measured the shear viscosities $(\eta)$ of the solutions using a rheometer (MCR 502 with CP50-1, Anton Paar), and the relaxation times $(\lambda)$ using extensional thinning in a pendant drop geometry (e.g., Fig. 7.1a) [188]. Calibrations are provided in appendix 7.8.1. A ratio of time scales related to the material properties of the system can be defined as the material Deborah number $\operatorname{De}_{\mathrm{m}}=\lambda / \tau$, where $\tau=\sqrt{\rho R^{3} / \gamma} \approx 5.8 \mathrm{~ms}$ is the inertiocapillary time scale based on the liquid density $\rho$, drop size $R$ and surface tension $\gamma$. In our experiments $\mathrm{De}_{\mathrm{m}}$ ranges from 0.08 to 10 . We note, however, that the relevant deformation rate during the early stages of coalescence scales as $1 / t$, so that a more significant ratio is the "local instantaneous Deborah number" $\lambda / t$, which reaches values up to $\mathcal{O}\left(10^{4}\right)$ at the smallest time scale that we can experimentally resolve. A strong polymer stretching and elastic 
effect is, therefore, anticipated during the early stages of coalescence.

Typical snapshots of coalescence experiments are shown in Fig. 7.2 for "spherical" and "sessile" geometries. In both cases, two symmetric drops are generated on needles (inner and outer diameters $0.52 \mathrm{~mm}$ and $0.82 \mathrm{~mm}$, respectively) by a syringe pump (PHD 2000, Harvard Apparatus). They are brought into contact by very slowly increasing the drop volume, such that the approach velocity (in the present experiments, three orders smaller than the typical bridge velocity) can be neglected [50]. The coalescence dynamics are recorded by a high speed camera (Nova S12, Photron, with $12 \mathrm{X}$ zoom lens, Navitar), allowing for frame rates up to 200000 frames per second, and resolutions down to $1 \mu \mathrm{m} /$ pixel. For sessile drops, we use glass substrates
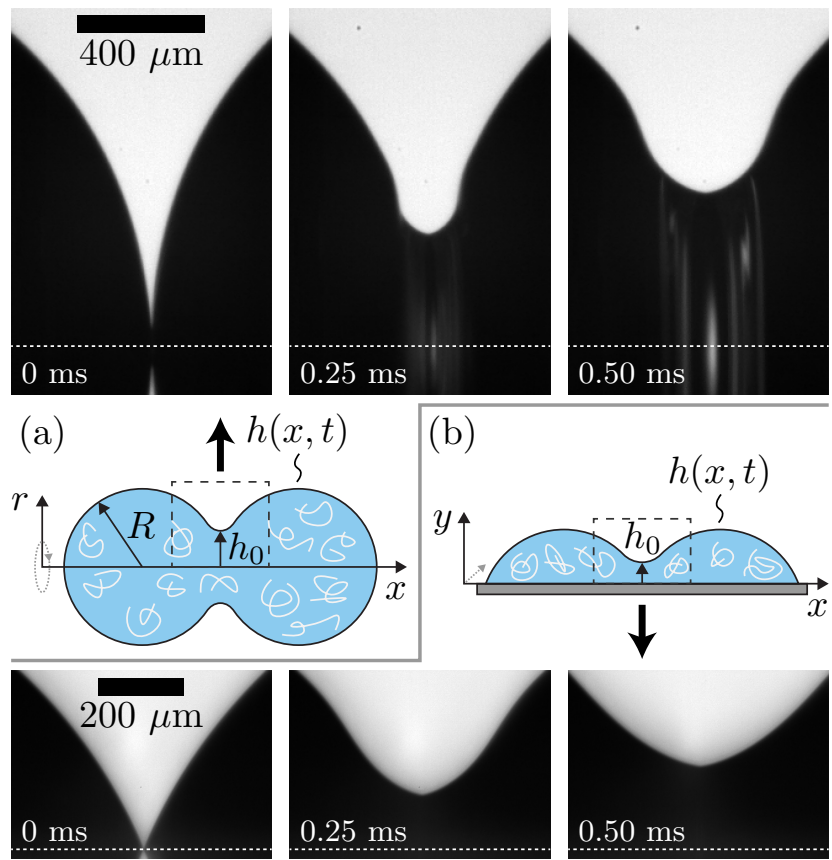

$x, t)$
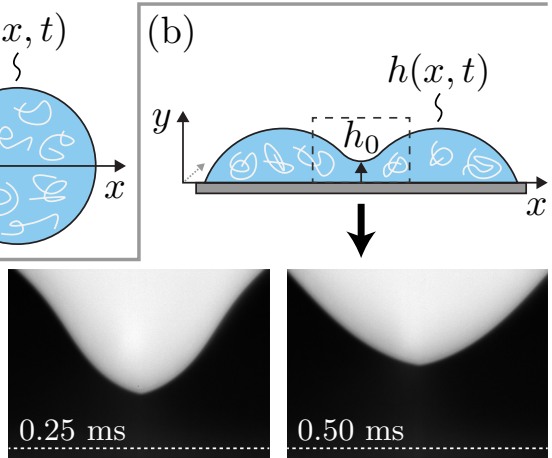

Figure 7.2: Coalescence of viscoelastic drops (PEO solution with concentration $1.0 \mathrm{wt} \%$ in two geometries: (a) "spherical", consisting of two freely suspended drops, and (b) "sessile", consisting of drops on a substrate. The three snapshots show a close-up of the bridge at three different times. The central bridge heigh $h_{0}(t)$ is defined with respect to the white dashed lines, indicating (a) $r=0$, and (b) $y=0$. 


\subsection{Time}

(Menzel-Gläser) that are made hydrophobic, and we use data with contact angles $45^{\circ} \leq \theta \leq 55^{\circ}$. We focus on the spatio-temporal evolution of the bridge shape, $h(x, t)$, as defined in Fig. 7.2. This profile is extracted using a custom sub-pixel interface tracking code. Finding the initial time of coalescence from direct imaging is challenging, yet important to determine the coalescence exponent $\alpha$. Here $t=0$ is determined by extrapolating a power-law $h_{0} \propto t^{\alpha}$, c.f., appendix 7.8.2.

\subsection{Time}

The temporal evolution of the bridge growth is only mildly affected by the presence of polymers. This is evident from the data in Fig. 7.3. In Fig. 7.3a we show the growth of the minimal bridge radius $h_{0}(t)$ for spherical drops over the full range of polymer concentrations. The data nearly fall on top of one another, and closely follow the dynamics of pure water (included as dark blue symbols). A weak trend is observed with increasing polymer concentration, leading to slightly slower dynamics. Importantly, however, the exact same power-law growth $h_{0} \propto t^{\alpha}$ is found for all concentrations. The fitted values of $\alpha$, the coalescence exponent, are shown in Fig. 7.3b-all polymer concentrations are consistent with $\alpha \approx 1 / 2$, which is the exponent for Newtonian coalescence of low-viscosity spherical drops $[45,58,59]$.

A similar behaviour is observed for sessile drops, see Fig. 7.3c-d. The data in panel Fig. 7.3c appear more scattered, which we attribute to the "wetting" nature of these experiments, for it is known that the variability of the contact angle at the moment of coalescence affects the pre-factor of the powerlaw growth $[42,44]$. Once again, however, the coalescence exponent is close to that of pure water, which for sessile drops is $\alpha=2 / 3$ [50]. Only the highest concentration exhibits a true departure from $2 / 3$, giving a lower exponent. Importantly, however, up to concentrations of $1 \mathrm{wt} \%$ there is no measurable effect of polymers on the coalescence exponent. We recall that a minute amount of polymer (down to $0.001 \mathrm{wt} \%$ ) is already sufficient to dramatically change the breakup of drops, from algebraic to exponential thinning [191]. It is therefore truly remarkable that the coalescence exponent is completely unaffected by the presence of polymers at such high concentrations. 

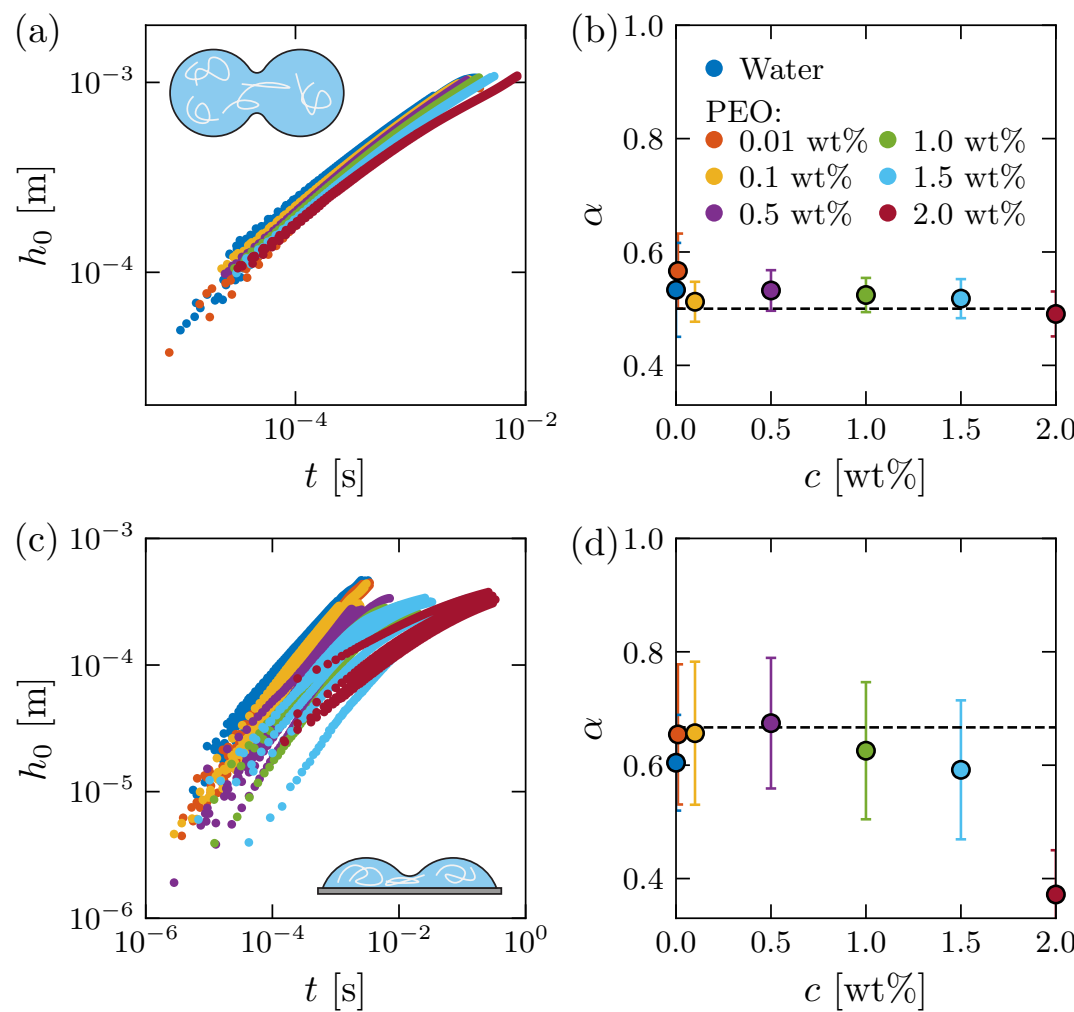

Figure 7.3: Temporal coalescence dynamics. (a) Minimum bridge radius $h_{0}$ as a function of time for spherical drop coalescence, and (b) the fitted exponent $\alpha$; the dashed line indicates $\alpha=1 / 2$. (c) Minimum bridge height $h_{0}$ as a function of time for sessile drop coalescence $\left(45^{\circ} \leq \theta \leq 55^{\circ}\right)$, and (d) the fitted exponent $\alpha$; the dashed line indicates $\alpha=2 / 3$. The error bars on $\alpha$ arise from a combination of the uncertainty in determining $t=0$, and the averaging of different experiments with the same liquid.

\subsection{Space}

Polymer stretching fundamentally alters the stress singularity during coalescence, and changes the spatial structure of the bridge. Indeed, one observes a dramatic difference in bridge curvature in Fig. 7.1b-c. Laplace's law of capillarity implies that the enhanced curvature for PEO solutions is due to strong polymer stresses. This polymer stretching in fact leads to a breakdown 

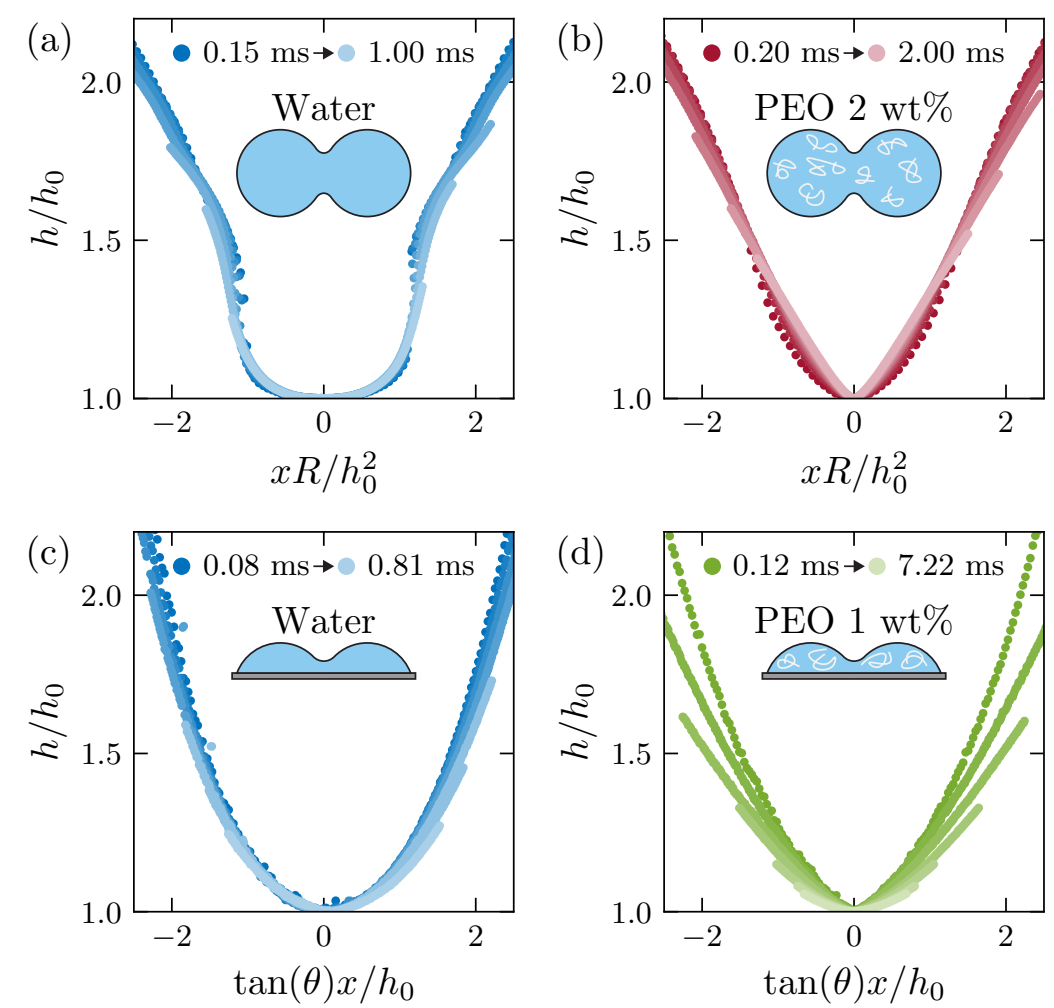

Figure 7.4: Spatial coalescence dynamics, for spherical drops (a, b) and sessile drops (c, d). Bridge profiles at different times are rescaled according to the Newtonian scaling laws. (a,c) For pure water, the rescaled bridge profiles collapse. (b, d) A breakdown of this Newtonian self-similarity is found for PEO solutions. (Spherical: 2 wt\% PEO, $t / \lambda=0.003 \rightarrow 0.03$, sessile: $1 \mathrm{wt} \%$ $\mathrm{PEO}, t / \lambda=0.004 \rightarrow 0.25$.)

of the self-similarity observed for the coalescence of pure water drops. Figure 7.4 shows bridge profiles $h(x, t)$ at various times, scaled according to the Newtonian similarity solutions. The spherical and sessile cases have the same vertical scaling $h / h_{0}$, but call for a different scaling of the horizontal position, respectively, as $x R / h_{0}^{2}$ for spherical drops of radius $R$, and as $\tan (\theta) x / h_{0}$ for sessile drops with contact angle $\theta[50]$.

Figure 7.4a and c correspond to the reference cases of pure water. The scaled profiles exhibit a collapse, revealing the self-similar nature of Newto- 
nian coalescence. Figure $7.4 \mathrm{~b}$ and $\mathrm{d}$ report the corresponding data for PEO solutions, scaled in this Newtonian way. The selected data correspond to the highest concentrations for which we still observed the Newtonian coalescence exponents $(2.0 \mathrm{wt} \%$ for the spherical case, and $1.0 \mathrm{wt} \%$ for the sessile case- see Fig. $7.3 \mathrm{~b}$ and d; for completeness, results for other concentrations are reported in appendix 7.8.3). The coalescence profiles for PEO no longer collapse with these scalings. The breakdown of Newtonian self-similarity emphasises the importance of polymeric stresses, which we now elucidate.

\subsection{Viscoelastic singularity}

An explicit analysis of the elastic singularity can be performed for sessile drops, where the geometry near coalescence is that of a wedge with angle $\theta$ as sketched in Fig. 7.5a, and the ensuing flow structure is considerably simple $[42,44,50]$. The wedge geometry near the point of coalescence has only a single length scale $h_{0}$, and is more amenable to analytical treatment than a spherical one which involves a distinct, horizontal scale $h_{0}^{2} / R$. The wedge geometry for small $\theta$ offers a further simplification, which is exploited below.

We start by noting that at the centre of the bridge $(x=0)$, the flow is nearly purely extensional in the vertical direction, with an extensional rate $\dot{h}_{0} / h_{0} \sim 1 / t$ that is very large at early times. The stress at $x=0$ at early times, thus reads

$$
\sigma \sim \bar{\eta}_{\infty} \frac{\dot{h}_{0}}{h_{0}} \sim \frac{\bar{\eta}_{\infty}}{t}
$$

where $\bar{\eta}_{\infty}$ is the extensional viscosity at high rates $[200,201]$. At such high stresses, non-linear polymer relaxation becomes important and can be captured by constitutive relations such as the FENE-P or the Giesekus model.

The amount of stretching will be much less pronounced away from $x=0$ (c.f., Fig. 7.5a), and we wish to identify the characteristic distance $\ell$ over which the singular polymer stress Eq. 7.1 decays. The central region of high polymer stretching is bordered by a region where polymer relaxation is negligible for $t / \lambda \ll 1$. This is a purely elastic region, where the polymer stretch can be found kinematically from the flow field [190]. Specifically, this involves comparing the height near $x=0$ to the original height $\theta x$ prior to deformation. For small angles, the resulting polymer stress reads (c.f., appendix 7.8.5)

$$
\sigma \sim \frac{G h_{0}}{\theta x}, \text { for } t \ll \lambda,
$$




\subsection{Viscoelastic singularity}
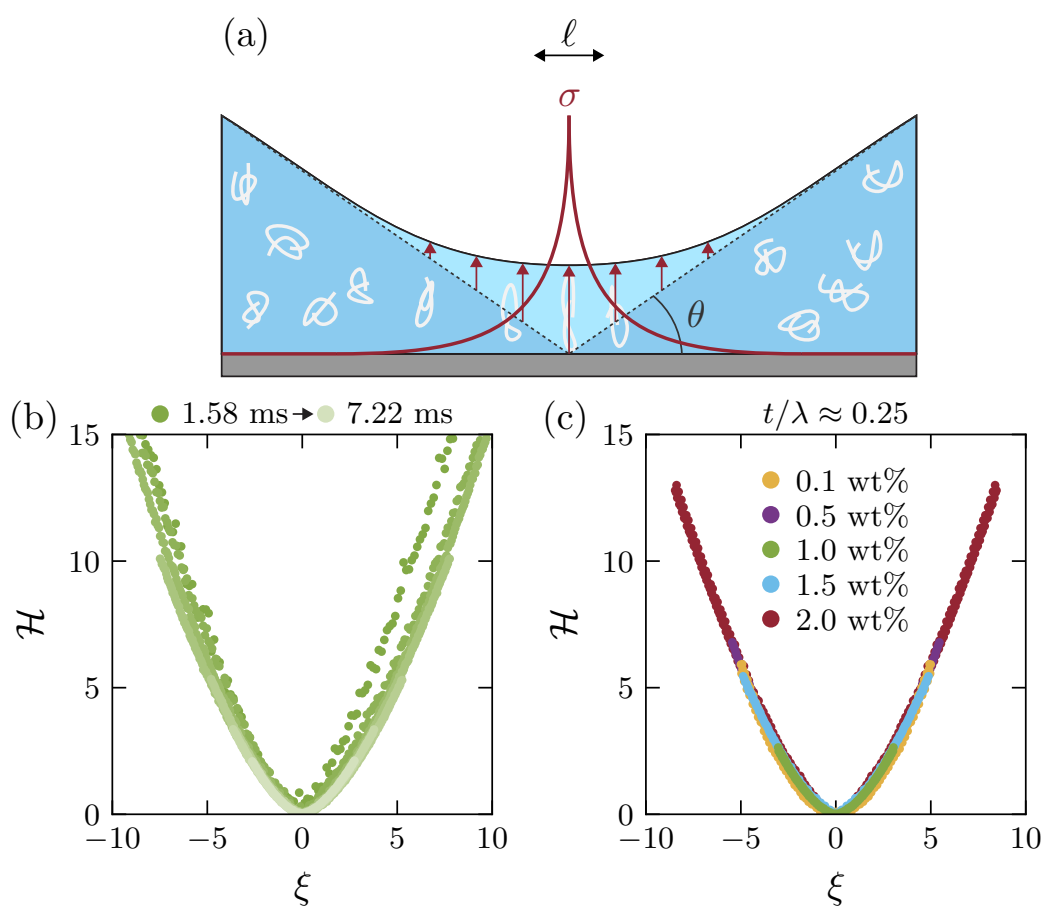

Figure 7.5: Self-similarity of sessile viscoelastic coalescence. (a) Schematic of the elastic singularity in wedge coalescence due to polymer stretching in the vertical direction. The singular polymer stress (red line) decays over a characteristic distance $\ell$ that is small compared to the bridge width. (b, c) Bridge profiles scaled according to the viscoelastic self-similar prediction Eq. 7.4 for (b) different times (PEO $1.0 \mathrm{wt} \%$ ), and (c) different PEO concentrations at $t / \lambda \approx 0.25$ (profiles symmetrised by averaging left-right). The data collapse on top of each other.

where the appearance of $G$, the elastic modulus of the medium, underlines the purely elastic nature. The extent $x \approx \ell$ of the central bridge region can be found by matching stresses in Eqs. 7.1-7.2. This gives

$$
\frac{G h_{0} t}{\theta \bar{\eta}_{\infty}} \sim \frac{h_{0} t}{\theta \lambda} \equiv \ell,
$$

where we have used that $\bar{\eta}_{\infty} / G \sim \lambda$ to express the result in terms of the calibrated relaxation time. We note here that $\ell$ is much smaller, by a factor $t / \lambda$, than the horizontal coalescence scale $h_{0} / \theta$ for pure water. 


\subsection{Viscoelastic self-similarity}

The emergence of the length scale $\ell$ explains the breakdown of the self-similarity in Fig. 7.4, and provides a new horizontal scale for viscoelastic coalescence. However, we still need to identify the appropriate vertical scale. This is done by balancing the polymer stress, $\bar{\eta}_{\infty} / t$, to the capillary pressure, $\gamma h^{\prime \prime}$ in the bridge region, which leads us to

$$
h(x, t)-h_{0} \sim \frac{t h_{0}^{2} G}{\lambda \gamma} \mathcal{H}(\xi), \quad \text { with } \quad \xi=\frac{x}{\ell} .
$$

This predicts a new self-similar regime where the bridge is described by a universal shape $\mathcal{H}(x / \ell)$.

To test these scaling predictions, we accordingly rescale the experimental data. Figure $7.5 \mathrm{~b}$ shows the result for the $1.0 \mathrm{wt} \%$ solution (showing the same experiment as in Fig. 7.4d). The data now exhibit a very good collapse which validates the emergence of the new, viscoelastic, self-similarity. We also compare the results from different polymer concentrations in Fig. 7.5c, each taken at a dimensionless time $t / \lambda \approx 0.25$ (arbitrarily chosen, while keeping the experimental resolution in mind). Since $G \lambda / \bar{\eta}_{\infty}$ in Eq. 7.4 is expected to vary with polymer concentration, the scaling of vertical axis involves an adjustable parameter (reported in appendix 7.8.4), while the horizontal scale $\ell$ is left parameter-free. An excellent collapse can indeed be obtained, as seen in Fig. 7.5c.

\subsection{Conclusion and outlook}

Elasticity affects drop coalescence in a remarkable fashion. While the spatial structure of the bridge is affected in a fundamental way, with sharp bridge profiles induced by polymer stretching, the temporal coalescence exponent remains unaffected. This second feature can be rationalised from our similarity analysis for sessile drops. The polymer stress in coalescence remains confined to a very narrow region, whose size depends on the "local instantaneous Deborah number" $\lambda / t$. This narrow region turns out insufficient to alter the inertio-capillary coalescence exponent. This scenario is markedly different from drop breakup for which polymer elasticity acts everywhere along the elongated filament. In breakup, the effective local Deborah number (relaxation time times the elongation rate) approaches a constant value along the entire thread, and therefore is able to dictate the temporal evolution [39]. 


\subsection{Conclusion and outlook}

As a future perspective, the next step would be to identify the structure in spherical drop coalescence. The spherical case lacks a slender limit and is therefore more intricate, also due to the two length scales that appear in already in the Newtonian case. It would be of interest to numerically investigate the initial phase of coalescence using different types of constitutive relations and different coalescence geometries. Our experiments, however, show that the scenarios for sessile and spherical drops are qualitatively similar. These findings will be important for a plethora of applications involving the merging of polymeric drops. 


\subsection{Appendix}

\subsubsection{Rheological characterisation}

A stock solution of polyethylene oxide (PEO, Sigma-Aldrich) with $2 \mathrm{wt} \%$ concentration was prepared by weighing and mixing PEO $\left(M_{W}=4 \times 10^{6} \mathrm{~g} / \mathrm{mol}\right)$ into water (MilliQ, Millipore Corporation). Lower concentrations (down to $0.01 \mathrm{wt} \%$ ) were achieved by dilution of the stock solution. The physical properties of the fluid were characterised using two methods. The shear viscosity $\eta$ was measured using a rheometer (MCR 502 with CP50-1 cone-plate geometry spindle, Anton Paar) and the resulting values are shown in Fig. 7.6 as a function of the shear rate $\dot{\gamma}$. The viscosity measurements were complemented by pendant drop experiments, which provide values for the surface tension $\gamma$ as well as the relaxation time $\lambda$ of the solutions. The surface tension, deduced by fitting the drop shape as in [202], is found to be insensitive to the polymer concentration: $\gamma=65 \pm 2 \mathrm{mN} / \mathrm{m}$. The relaxation time $\lambda$ was measured by studying the thread thinning dynamics [188]. An example snapshot of a thread is shown in Fig. 7.1a. We performed at least five measurements per solution to extract the minimum thread diameter $d(t)$. We show in Fig. 7.7a the thread time evolution on a semi-log scale, which is fitted with the function $d / d_{\text {needle }} \sim \exp (-t / 3 \lambda)$, where $d_{\text {needle }}$ denotes the dispensing needle diameter and $t$ is the time. Figure 7.7b shows the deduced average relaxation times $\lambda$ as a function of the PEO concentration.

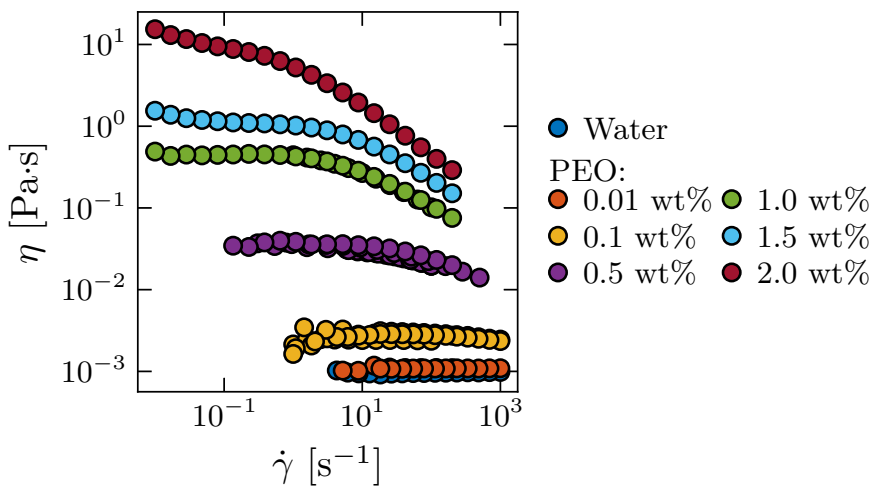

Figure 7.6: Viscosity $\eta$ as a function of shear rate $\dot{\gamma}$ for various PEO concentrations. Data were averaged for a few seconds for the high shear rates to a few minutes for the low shear rates. 


\subsection{Appendix}
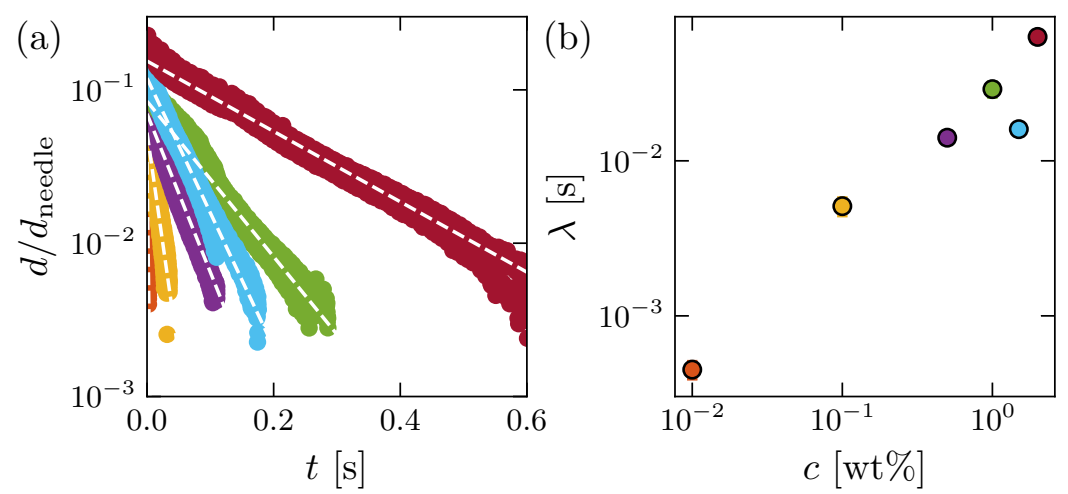

Figure 7.7: (a) Thinning dynamics of a thread for various PEO solutions. The thread has minimum diameter $d$. At least five experiments are shown for each solution. The white dashed lines show fits of the type $d / d_{\text {needle }} \sim \exp (-t / 3 \lambda)$. (b) Relaxation time $\lambda$ as a function of PEO concentration $c$, as determined from the data in (a).

\subsubsection{Finding the initial moment of drop contact}

The accurate fitting of the exponent $\alpha$, defined as $h_{0} \propto\left(t-t_{0}\right)^{\alpha}$, relies on a good determination of $t_{0}$, i.e., the moment of first contact between the coalescing drops. A slightly misdefined $t_{0}$ leads to curved data on a log-log plot, affecting the fitted $\alpha$. Typical coalescence data for the sessile case obtained from optical microscopy is shown in Fig. 7.8a-b. The exact moment of first contact is difficult to determine from the experimental images - pixel resolution limits the visibility of small bridges, the moment of first contact can be in between two frames, and sideview images can lead to apparent contact before the actual contact. The method by which we determine the precise moment of $t_{0}$ is illustrated by the example measurement shown in Fig. 7.8. We manually selected a range, starting at a frame where the coalescence had clearly started and ending at sufficiently small $t$ such that the growth is expected to follow a power law. For various small time shifts, we then checked if the selected data fits to a power law $h_{0} \propto\left(t-t_{0}\right)^{\alpha}$, by fitting (least squares method) a straight line where $\alpha$ was a free parameter. The value of $t_{0}$ was then determined by selecting the (small) time shift which resulted in the smallest fit residue. Fig. $7.8 \mathrm{c}-\mathrm{d}$ show the result of our method for the data shown in Fig. $7.8 \mathrm{a}-\mathrm{b}$. This procedure leaves $\alpha$ as a free parameter, which is reported in the main text. 


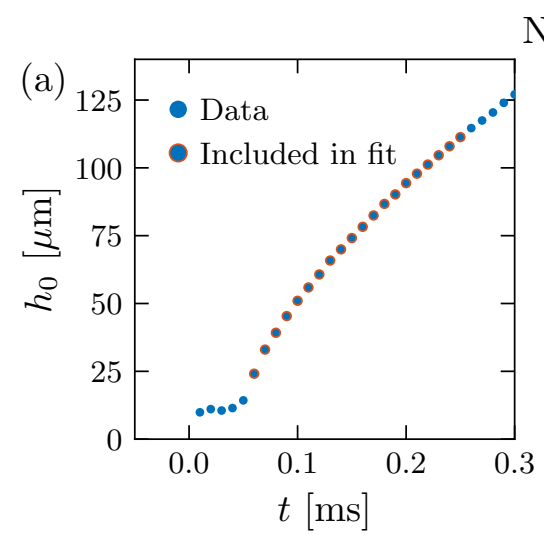

No shift

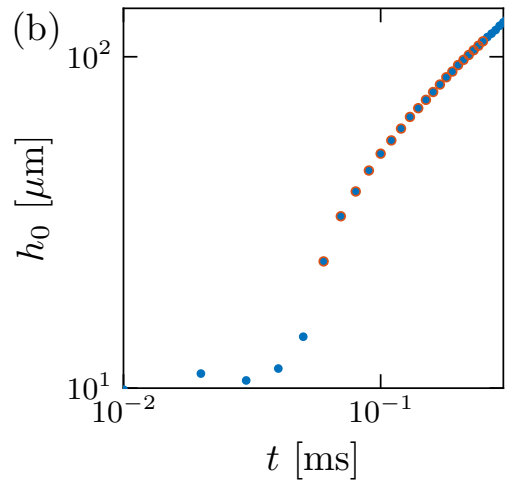

Shift
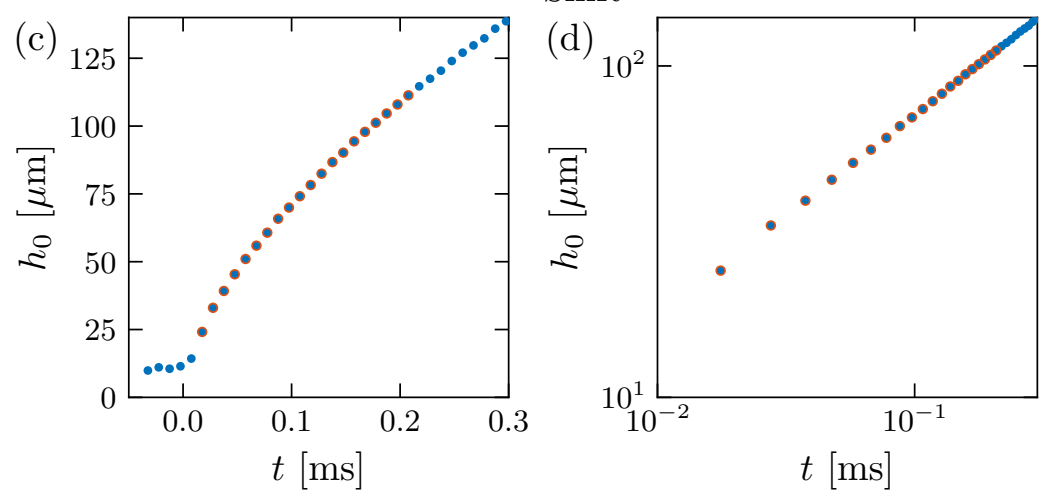

Figure 7.8: Minimum bridge height $h_{0}$ as a function of time, with misdefined $t_{0}$ on (a) linear axes, and (b) log-log axes. The same data is shown with corrected $t_{0}$ on (c) linear axes, and (d) log-log axes.

\subsubsection{Additional data for various PEO concentrations}

In the main text, we present bridge profiles scaled by the Newtonian selfsimilar rescaling (Fig. 7.4) and by the newly proposed viscoelastic self-similar rescaling (Fig. 7.5). The main text shows profiles for selected concentrations and selected times. For completeness, here we provide the full set of data (scaled and unscaled) for all concentrations at various times. Figure 7.9 gives the data for the sessile drop coalescence. Figure 7.10 gives the data for the spherical drop coalescence. Note how the Newtonian collapse progressively deteriorates with increasing $\mathrm{PEO}$ concentration. 

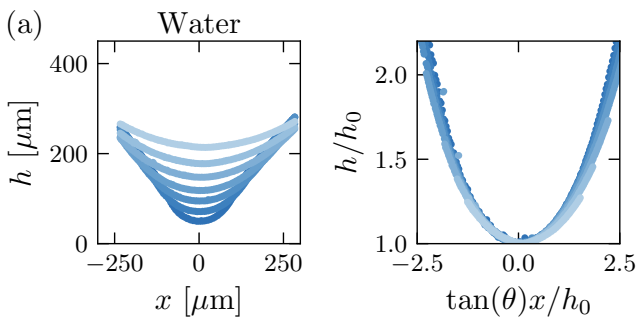

- $0.08 \mathrm{~ms}$

- $0.14 \mathrm{~ms}$

- $0.22 \mathrm{~ms}$

- $0.31 \mathrm{~ms}$

$0.44 \mathrm{~ms}$

- $0.60 \mathrm{~ms}$

$0.81 \mathrm{~ms}$
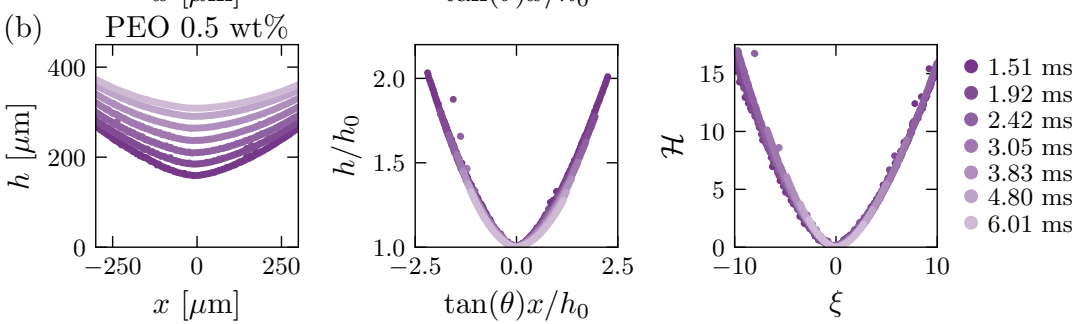

(c)
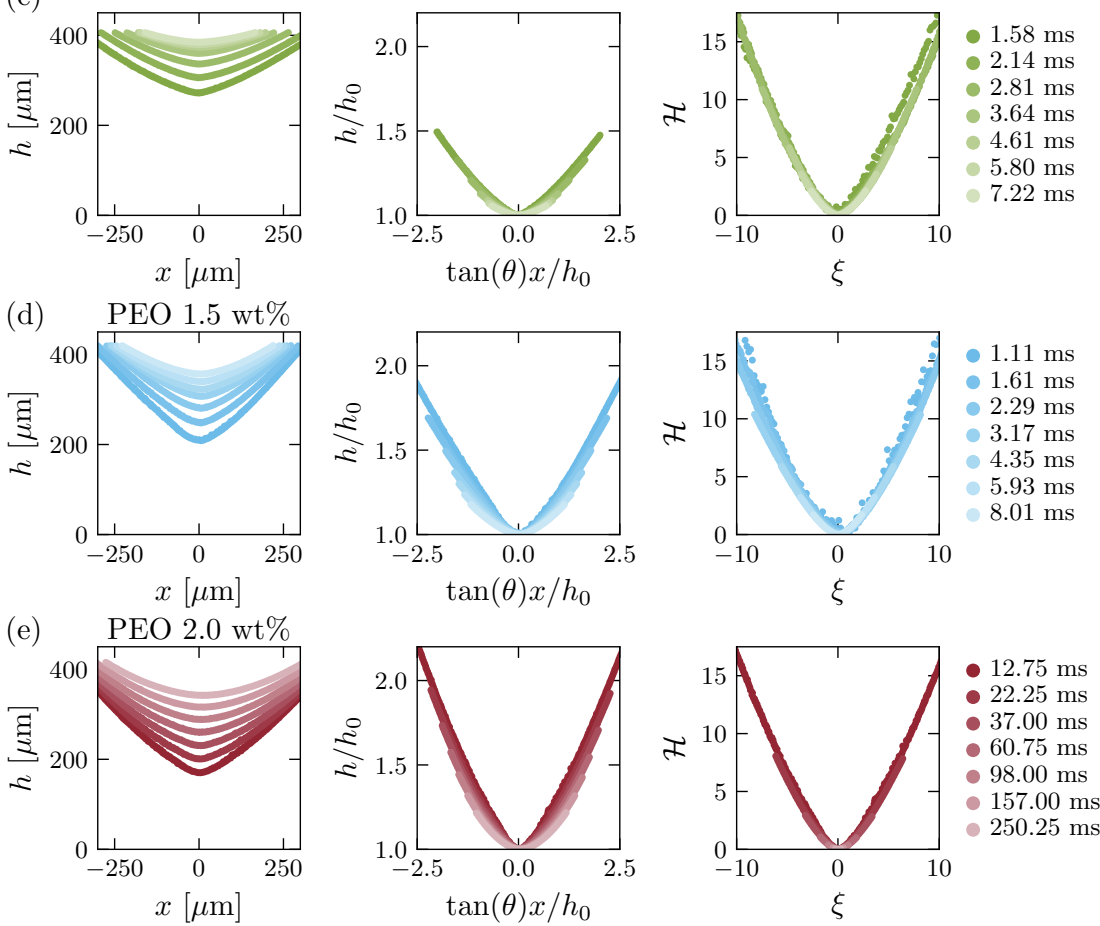

Figure 7.9: Raw data and scaled data for sessile coalescence for various additional PEO concentrations. The leftmost column shows raw data, the middle column shows data scaled according to the Newtonian similarity scaling, the rightmost column shows the data scaled according the viscoelastic similarity scaling. The Newtonian collapse progressively deteriorates with increasing PEO concentration. 

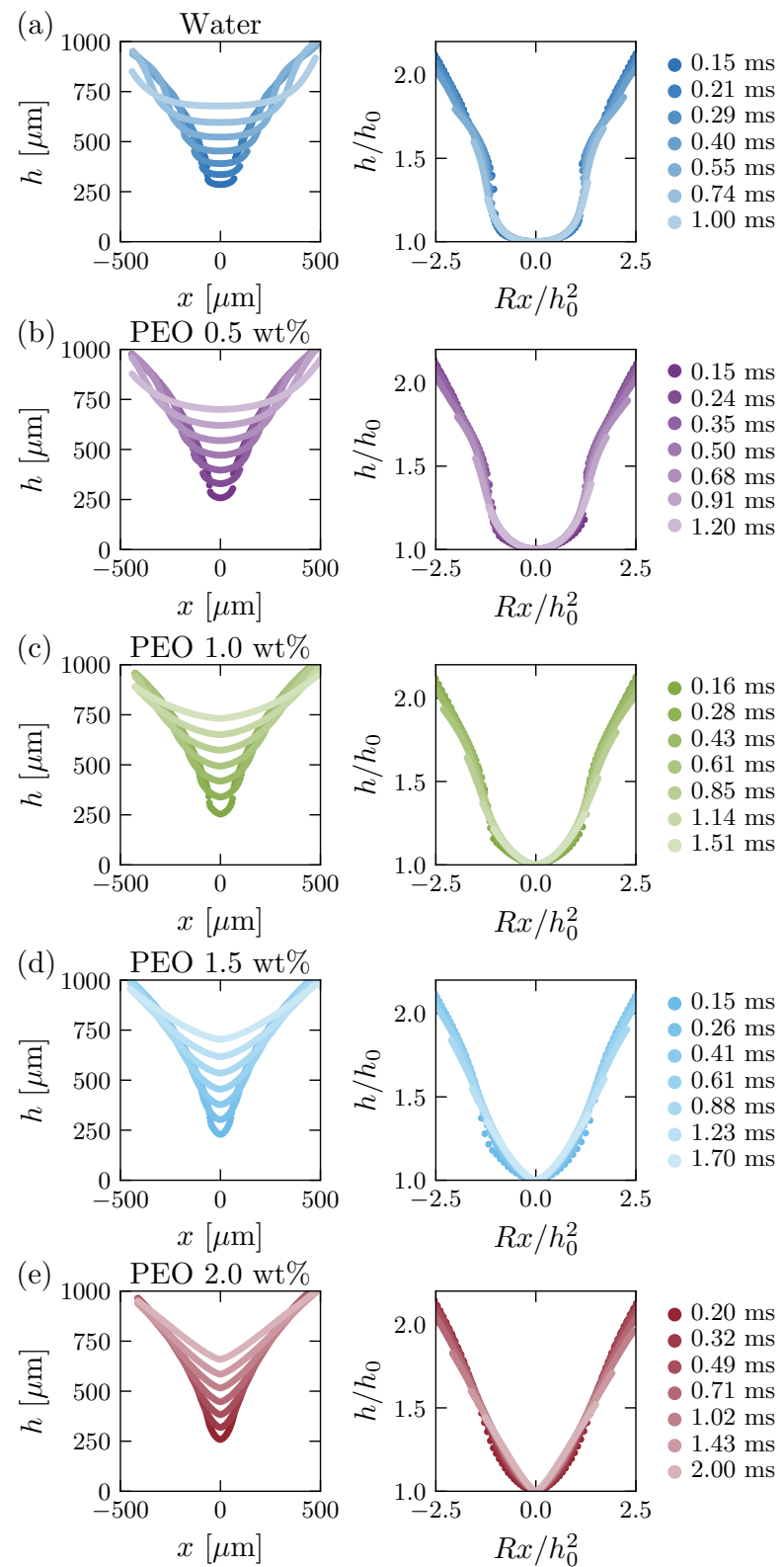

Figure 7.10: Raw data and scaled data for spherical coalescence for various additional PEO concentrations. The left column shows raw data, the right column shows data scaled according to the Newtonian similarity scaling. The Newtonian collapse progressively deteriorates with increasing PEO concentration. 


\subsection{Appendix}

\subsubsection{Pre-factor to Eq. 7.4}

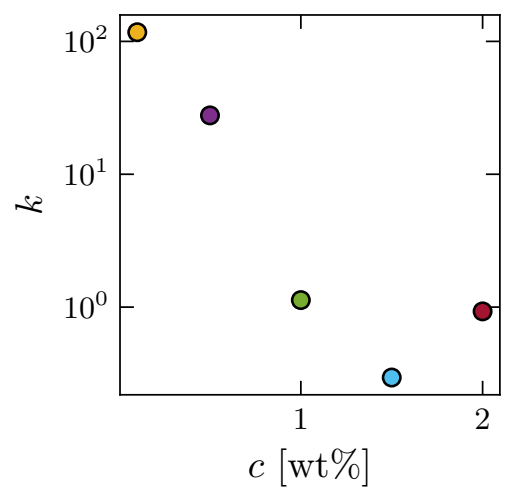

Figure 7.11: The collapse of profiles in Fig. 7.5c involved a pre-factor to the scaling law (Eq. 7.4). The plot reports this pre-factor $k$ for various concentrations.

\subsubsection{The wedge geometry and the purely elastic regime}

Here, we motivate in more detail the scaling law in the purely elastic regime presented in Eq. 7.2 of the main text. For a stress-free initial condition, the polymer stress without relaxation reads

$$
\boldsymbol{\sigma}^{p}=G \mathbf{F} \cdot \mathbf{F}^{T},
$$

where $\mathbf{F}$ is the deformation gradient tensor $\mathbf{F}=\partial \mathbf{x} / \partial \mathbf{X}$, and $\mathbf{x}$ and $\mathbf{X}$ are the Eulerian and Lagrangian coordinates, respectively (see Fig. 7.12) [190]. In the absence of polymer relaxation, the components of $\mathbf{A}=\mathbf{F} \cdot \mathbf{F}^{T}$ along principal directions give the square of the principal stretch that is induced by the flow.

In sessile drop coalescence, the predominant stretch near the centre of the bridge is in the vertical direction. We thus need to evaluate $A_{y y}$. Furthermore, in the slender limit and ignoring a thin viscous boundary layer near the wall, the flow will be approximately homogeneous across the film thickness [171]. The stretching can then be evaluated by comparing $h(x, t)$ to the reference height $\theta X$ before the start of the coalescence, leading to

$$
A_{y y} \approx\left(\frac{h(x, t)}{\theta X}\right)^{2} \approx\left(\frac{h_{0}(t)}{\theta X}\right)^{2} .
$$


The second relation comes from an evaluation near the point of coalescence. Mass conservation in the two shaded regions in Fig. 7.12 gives

$$
\frac{1}{2} X^{2} \theta=\int_{0}^{x} h(x, t) d x \approx h_{0}(t) x .
$$

From the two relations one obtains $A_{y y} \sim h_{0} / \theta x$. Therefore, the corresponding stress component $\sigma_{y y} \sim G h_{0} / \theta x$. This is Eq. 7.2 of the main text.

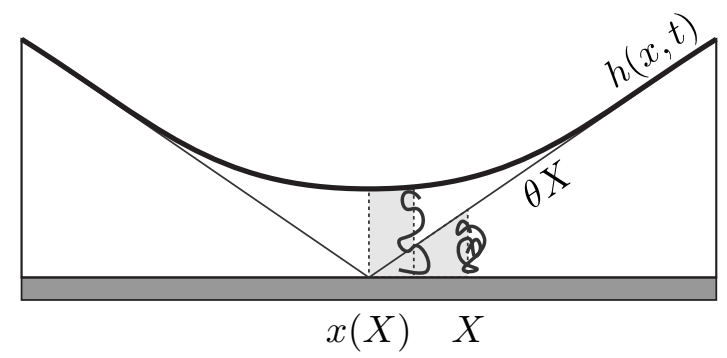

Figure 7.12: Schematic of sessile viscoelastic coalescence in the bridge region. 


\section{Chapter 8}

\section{The asymmetric coalescence of two droplets with different surface tensions is caused by capillary waves}

When two droplets with different surface tensions collide, the shape evolution of the merging droplets is asymmetric. Using experimental and numerical techniques, we reveal that this asymmetry is caused by asymmetric capillary waves, which are the result of the different surface tensions of the droplets. We show that the asymmetry is enhanced by increasing the surface tension difference, and suppressed by increasing the inertia of the colliding droplets. Furthermore, we study capillary waves in the limit of no inertia. We reveal that the asymmetry is not directly caused by Marangoni forces. In fact, somehow counterintuitive, asymmetry is strongly reduced by the Marangoni effect. Rather, the different intrinsic capillary wave amplitudes and velocities associated with the different surface tensions of the droplets lie at the origin of the asymmetry during droplet coalescence.

@ Published as: Michiel. A. Hack, Patrick Vondeling, Menno Cornelissen, Detlef Lohse, Jacco H. Snoeijer, Christian Diddens, and Tim Segers, Asymmetric coalescence of two droplets with different surface tensions is caused by capillary waves, Phys. Rev. Fluids 6, 104002 (2021). 
Chapter 8. Asymmetric coalescence is caused by capillary waves

\subsection{Introduction}

The collision and subsequent coalescence of liquid droplets is omnipresent in both nature and technology. For example, small droplets collide in the atmosphere, forming larger ones that eventually fall to the Earth as rain [155]. The coalescence of ink droplets is also a vital process in inkjet printing, the collisions and coalescence of ethanol and diesel droplets play an important role in combustion engines, and the in-air coalescence of droplets has recently been introduced as a method for the mass production of functional micromaterials $[7-9,68,203-206]$. The fundamental importance of droplet collision and coalescence has led to intense investigation in recent decades $[42,43,57,59,207]$. These studies found that the collision and coalescence dynamics of identical droplets are determined by both the geometry and composition of the droplets $[44,45,50]$. It is therefore expected that these dynamics are strongly altered when the two droplets are not identical. Indeed, unequal-sized droplets show different collision outcomes when compared to collisions between droplets of equal size $[62,208]$. Moreover, when two droplets of different surface tensions coalesce, they show fundamentally different dynamics than their equal-surface tensions counterpart - they exhibit intricate phenomena such as 'delayed coalescence' and have enhanced internal mixing [46, 49, 52, 53, 64, 209].

In this chapter, we study the mid-air collision of two droplets with different surface tensions. The difference between the surface tensions of the droplets induces a tangential Marangoni stress at the droplet interface, which results in the engulfment of the higher surface tension droplet by the lower surface tension liquid [63,210]. A similar system was studied by Gao et al., and later by Kohno et al., who reported that the shape of such droplets during their coalescence is asymmetric [211-213]. Gao et al. and Kohno et al. attribute the asymmetric droplet shape to capillary wave interference [211-213]. In their model, a capillary wave forms at the point of contact of the two droplets, and subsequently travels over the high surface tension droplet's interface, driven by the difference in surface tensions between the two droplets, i.e., due to the Marangoni effect. When the capillary wave reaches the droplet apex, it constructively interferes with itself, forming a protrusion, thus resulting in an asymmetric droplet shape. Capillary waves also form and interfere during the coalescence of identical droplets, though in that case the protrusions that appear are identical on both droplets $[43,56,59,61,62]$. Understanding the dynamics of engulfment and capillary waves in the presence of the Marangoni effect, and in particular their interaction in droplet collisions, remains an open question. 


\subsection{Methods}

This work offers a detailed study of the asymmetric evolution of the droplet shapes during the collision of two droplets with different surface tensions. Using a combination of experiments and numerical simulations, we show, presumably unsurprisingly, that the asymmetry grows as the difference between the surface tensions of the two droplets is increased. However, more remarkably, we show that this is not due to the Marangoni forces. Instead, we unravel the critical role of capillary waves in the formation of the asymmetric droplet shapes. Finally, we also reveal that the asymmetry can be suppressed by increasing the collisional Weber number.

\subsection{Methods}

\subsubsection{Experimental method}

The experimental setup is shown in Fig. 8.1a. Two piezo-electric droplet dispensers (AD-K-501, Microdrop Technologies) were used to generate droplets that collide in flight. A continuous stream of droplets was generated to prevent compositional changes in the liquid due to evaporation and fouling of the liquid interface at the nozzle exit of the dispenser. A high-speed camera (HPV-X2, Shimadzu) equipped with a $20 \times$ microscope lens (Olympus) was used to record the dynamics of the collisions with a spatial resolution of

(a) Top view

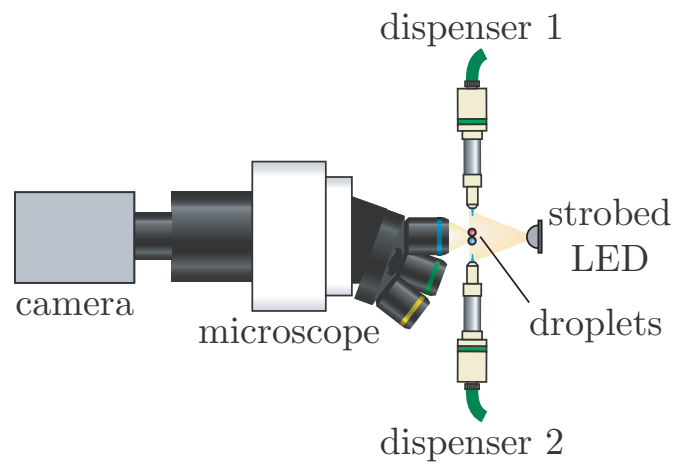

(b) Side view

dispenser 1 dispenser 2
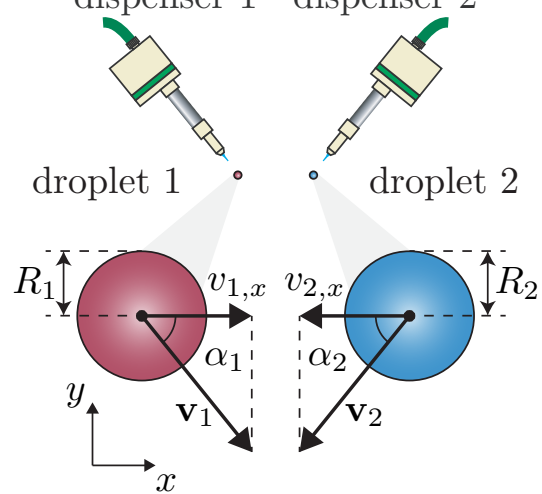

Figure 8.1: (a) Schematic of the experimental setup (top view). (b) Definitions of the collision parameters (side view). 
Table 8.1: Properties of the liquids used in the experiments of this study. The surface tensions are based on the values reported in Ref. [214]. The density and viscosity are based on the values reported in Ref. [215].

\begin{tabular}{lccc}
\hline Liquid & $\gamma[\mathrm{mN} / \mathrm{m}]$ & $\eta[\mathrm{mPa} \cdot \mathrm{s}]$ & $\rho\left[\mathrm{kg} / \mathrm{m}^{3}\right]$ \\
\hline water & 72.5 & 0.92 & 997 \\
8.0 wt\% ethanol in water & 51.3 & 1.51 & 986 \\
34.5 wt\% ethanol in water & 31.9 & 2.56 & 941 \\
ethanol & 22.9 & 1.17 & 789 \\
\hline
\end{tabular}

$1.6 \mu \mathrm{m} /$ pixel at one to two million frames per second. We used a strobed LED to generate high-intensity light flashes with a duration of $300 \mu$ s to illuminate the collisions. The duration of a single LED flash is longer than the collision time. The droplet dispensers were oriented at an angle to facilitate collisions in the imaging plane of the microscope. The impact velocity (see Fig. 8.1b) is therefore defined as $v=v_{1, x}+v_{2, x}=\cos \alpha_{1}\left\|\mathbf{v}_{1}\right\|+\cos \alpha_{2}\left\|\mathbf{v}_{2}\right\|$, where $\alpha_{1} \approx \alpha_{2}$ and $v_{1, x} \approx v_{2, x}$. The velocities $\left\|\mathbf{v}_{1}\right\|$ and $\left\|\mathbf{v}_{2}\right\|$ as well as the angles $\alpha_{1}$ and $\alpha_{2}$ were extracted from the movie frames captured prior to the collision event. The velocity of the droplets was controlled by the amplitude of the electric pulse applied to the piezo-electric actuator in the dispenser, resulting in impact velocities in the range $0.4 \mathrm{~m} / \mathrm{s} \leq v \leq 6.7 \mathrm{~m} / \mathrm{s}$. The radii of the droplets were $R_{1} \approx R_{2} \approx 35 \mu \mathrm{m}$ in all experiments.

The experiments were performed with water (MilliQ, Millipore Corporation), ethanol (99.8\% purity, Sigma Aldrich), and mixtures of water and ethanol. The properties of these liquids are summarised in Table 8.1. Water and ethanol were chosen for their large difference in surface tension, low viscosities, and miscibility. An estimated $0.8 \%$ of the initial droplet volume evaporates during the flight and collision of the droplets, such that the surface tensions of the droplets remain approximately constant during the experiment, see appendix 8.6.1 for details.

\subsubsection{Dimensionless groups}

We define the dimensionless surface tension difference $\tilde{\gamma}$ and collisional Weber number as

$$
\tilde{\gamma}=\frac{\gamma_{2}-\gamma_{1}}{\gamma_{1}}, \quad \text { and } \quad \mathrm{We}=\frac{\bar{\rho} v^{2} \bar{R}}{\gamma_{2}}
$$




\subsection{Methods}

respectively, where $\bar{\rho}=\left(\rho_{1}+\rho_{2}\right) / 2, \rho_{1}$ and $\rho_{2}$ are the densities, and $\gamma_{1}$ and $\gamma_{2}$ the surface tensions of droplets 1 and 2 , respectively, and $\bar{R}$ is the average radius of the droplets. We always choose $\gamma_{2}>\gamma_{1}$ by definition. In the present work, $\tilde{\gamma}$ and We were varied over the range of $0 \leq \tilde{\gamma} \leq 4$, and $0 \leq \mathrm{We} \leq 25$. The density ratio $\rho_{2} / \rho_{1}$ is an additional dimensionless parameter, which is determined by the values in Table 8.1.

The Ohnesorge number and Bond number are defined as

$$
\mathrm{Oh}=\frac{\eta_{2}}{\sqrt{\bar{\rho} \gamma_{2} \bar{R}}}, \quad \text { and } \quad \text { Bo }=\frac{\left(\bar{\rho}-\rho_{\text {air }}\right) g \bar{R}}{\gamma_{2}},
$$

respectively, where $\eta_{2}$ is the viscosity of droplet $2, g$ is the gravitational acceleration, and $\rho_{\text {air }}$ is the density of air. The gravitational and viscous forces can be neglected in all experiments and numerical simulations considered in the present work, since $\mathrm{Oh}=\mathcal{O}\left(10^{-2}\right) \ll 1$ and $\mathrm{Bo}=\mathcal{O}\left(10^{-4}\right) \ll 1$.

Finally, time is normalised by the inertio-capillary time scale

$$
\tilde{t}=\frac{t}{\tau}, \quad \text { with } \quad \tau_{i}=\sqrt{\frac{\rho_{i} R_{i}^{3}}{\gamma_{i}}},
$$

which is associated with the surface tension-driven deformation of the droplets during coalescence [216]. Here, $i \in[1,2]$ indicates that the properties of either droplet 1 or 2 are used, based on which is one being considered.

\subsubsection{Numerical method and its validation}

A numerical simulation of the collision process demands an accurate tracking of the interface and the treatment of the composition and velocity field, which are strongly coupled due to the Marangoni effect. To that end, an axisymmetric sharp-interface arbitrary Lagrangian-Eulerian (ALE) finite element method is employed. Both droplets are represented by a mesh consisting of triangular elements, using second order basis functions for the velocity $\mathbf{u}$ and linear basis functions for the pressure $p$ and composition $c$. Due to the low viscosity and density ratio, the gas phase is not considered. At higher impact velocities, the presence of the gas phase can however influence the collision due to the buildup of an air cushion, the formation of a dimple, and the presence of entrapped bubbles [217-219]. At lower collision velocities, however, hardly any dimple forms in collisions, and simulations with and without consideration of the gas phase lead to almost identical results. Evaporation is also disregarded in 
the numerical simulations, given the short time scale of the collision process compared to typical droplet evaporation time scales.

The Navier-Stokes equations and the convection-diffusion equation for the composition are solved, i.e.,

$$
\begin{aligned}
\rho\left(\partial_{t} \mathbf{u}+\mathbf{u} \cdot \nabla \mathbf{u}\right) & =-\nabla p+\nabla \cdot\left[\eta\left(\nabla \mathbf{u}+(\nabla \mathbf{u})^{\mathrm{T}}\right)\right], \\
\partial_{t} \rho+\nabla \cdot(\rho \mathbf{u}) & =0, \\
\rho\left(\partial_{t} c+\mathbf{u} \cdot \nabla c\right) & =\nabla \cdot(\rho D \nabla c),
\end{aligned}
$$

where $D$ is the diffusion coefficient. The properties $\rho, \eta, D$ and also the surface tension $\gamma$ are functions of the composition $c$. At the free interface, the Laplace pressure and the Marangoni shear stress are imposed, i.e.,

$$
\mathbf{n} \cdot \mathbf{T} \cdot \mathbf{n}=\kappa \gamma, \text { and } \mathbf{n} \cdot \mathbf{T} \cdot \mathbf{t}=\nabla_{\mathrm{S}} \gamma \cdot \mathbf{t},
$$

with the stress tensor $\mathbf{T}=-p \mathbf{1}+\eta\left(\nabla \mathbf{u}+(\nabla \mathbf{u})^{\mathrm{T}}\right)$, the normal $\mathbf{n}$, and tangent t. Here, $\kappa$ is the curvature of the interface and $\nabla_{\mathrm{S}}$ is the surface gradient operator. Finally, the kinematic boundary condition is enforced via a field of Lagrange multipliers, which ensures that the normal mesh motion at the interface coincides with the normal velocity $\mathbf{u} \cdot \mathbf{n}$, whereas the positions of the mesh nodes in the bulk follow the motion of a deformed pseudo-elastic body $[172,220]$.

The coalescence is initiated as follows. The moment the droplets approach each other within $1 \%$ of the average radius, the interfaces of both droplets are connected and the mesh is reconstructed, followed by an interpolation of the solved fields and their values of the previous time step, which is required for the second order time stepping via the backward differentiation formula. Note that $R_{1}=R_{2}$ unless otherwise stated. Mesh reconstruction is also invoked whenever the mesh quality suffers from strong deformations.

The method is implemented on the basis of the framework OOMPH-LIB [172]. All equations are solved with a monolithic Newton method using a direct solver for the inversion of the Jacobian. The implementation has already been successfully applied for various scenarios involving multi-component droplet dynamics and Marangoni flow, see e.g., Refs. [120,221]. The dependence of the results on the mesh size and the time step is discussed in appendix 8.6.2.

Figures 8.2 and 8.3 show the validation of our numerical method against our experimental results. The temporal evolution of the neck width $w$ for We $=0$ and $\tilde{\gamma}=0$ is shown in Fig. 8.2, and it is in good agreement with the expected scaling law $w \propto(\gamma R / \rho)^{1 / 4} t^{1 / 2}$ [57]. For comparison, we 


\subsection{Methods}

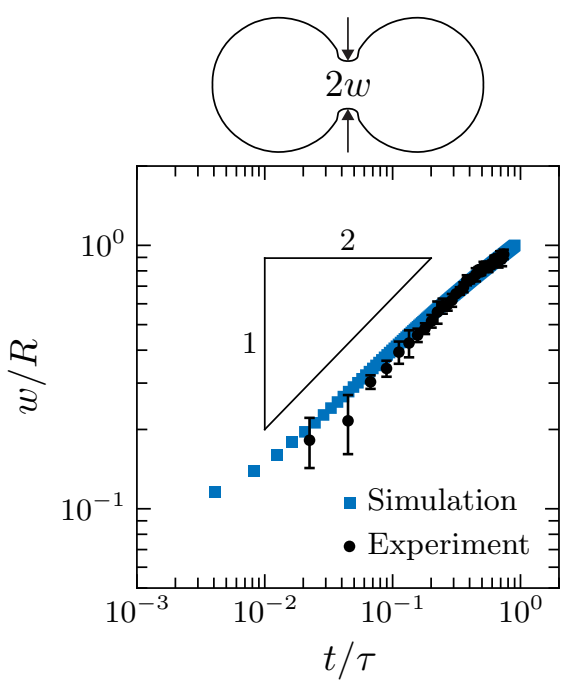

Figure 8.2: Temporal evolution of the neck width $w$ for two coalescing water droplets $\left(\gamma=72.5 \mathrm{mN} / \mathrm{m}, \rho=997 \mathrm{~kg} / \mathrm{m}^{3}\right.$, and $\left.\eta=0.92 \mathrm{mPa} \cdot \mathrm{s}\right)$. The experimental data is an average of five measurements with $\mathrm{We}=0.17$. The error bars indicate the standard deviation between the measurements at each time step.

show the experimentally obtained neck width for We $=0.17$ (due to the nature of the setup, we are unable to achieve $\mathrm{We}=0$ in the experiments) and find good agreement with the numerical simulations. A comparison of the coalescence dynamics for various We $>0$ and $\tilde{\gamma}$ is shown in Fig. 8.3, where the experimental parameters $v_{i}, R_{i}, \gamma_{i}, \eta_{i}$, and $\rho_{i}(i \in[1,2])$ were used as input for the numerical simulations. A detailed physical discussion of Fig. 8.3 is provided in section 8.3. The surface tension depends on the local composition $c$ as $\gamma=0.016367+0.007399 /(c+0.131824)$ (in $\mathrm{N} / \mathrm{m}$ ), which is an empirical fit to the data presented by Vazquez et al. [214]. The density $\rho=997.0+\left(-208.0\left(0.660 c+0.340 c^{2}\right)\right)\left(\right.$ in $\left.\mathrm{kg} / \mathrm{m}^{3}\right)$, viscosity $\eta=0.000923+0.00821 c-0.0111 c^{2}+0.00314 c^{3}$ (in $\left.\mathrm{Pa} \cdot \mathrm{s}\right)$, and diffusion coefficient $D=1.25 \times 10^{-9}\left(1.0-2.78 c+2.72 c^{2}\right)\left(\right.$ in $\left.\mathrm{m}^{2} / \mathrm{s}\right)$ were similarly obtained by empirical fits $[215,222]$. Here, $c=0$ indicates pure water and $c=1$ indicates pure ethanol. Excellent agreement between the experiments and numerical simulations demonstrates that our numerical method accurately describes the collision dynamics. No simulation is shown in Fig. 8.3d, 


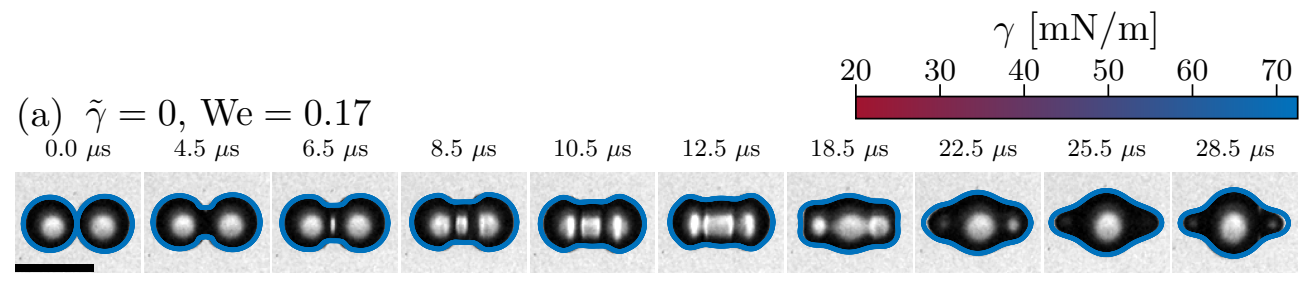

(b) $\tilde{\gamma}=0, \mathrm{We}=22.88$

$0.0 \mu \mathrm{s} \quad 2.0 \mu \mathrm{s} \quad 4.0 \mu \mathrm{s}$

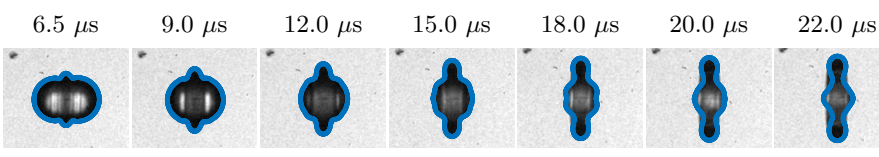

(c) $\tilde{\gamma}=2.17$, We $=0.40$

$0.0 \mu \mathrm{s} \quad 8.0 \mu \mathrm{s} \quad 10.0 \mu \mathrm{s} \quad 14.0 \mu \mathrm{s} \quad 18.0 \mu \mathrm{s} \quad 22.0 \mu \mathrm{s} \quad 26.0 \mu \mathrm{s} \quad 30.0 \mu \mathrm{s} \quad 42.0 \mu \mathrm{s} \quad 52.0 \mu \mathrm{s}$

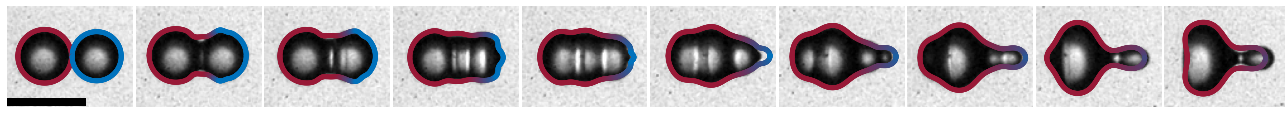

(d) $\tilde{\gamma}=2.17, \mathrm{We}=11.80$

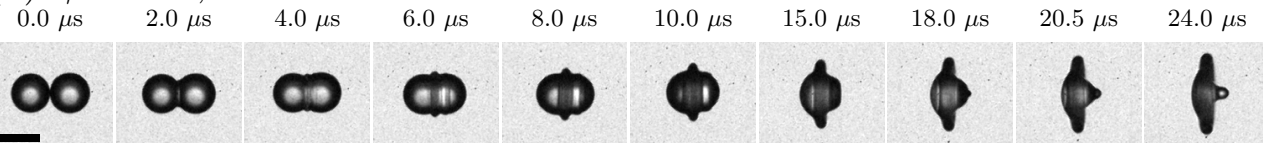

(e) $\tilde{\gamma}=1.27, \mathrm{We}=2.27$

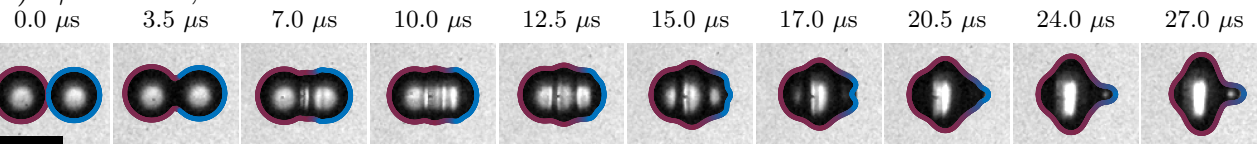

Figure 8.3: Direct comparison of experiments (images) and numerical simulations (coloured lines). The experimental parameters $v_{i}, R_{i}, \gamma_{i}, \eta_{i}$, and $\rho_{i}(i \in[1,2])$ were used as input for the numerical simulations. Collisions between (a) two water droplets with $\tilde{\gamma}=0$, We $=0.17$, Bo $=1.6 \times 10^{-4}$, $\mathrm{Oh}=1.8 \times 10^{-2}$, (b) two water droplets with $\tilde{\gamma}=0$, We $=22.88$, Bo $=2.2 \times 10^{-4}, \mathrm{Oh}=1.7 \times 10^{-2}$, (c) a water droplet and an ethanol droplet with $\tilde{\gamma}=2.17$, We $=0.40, \mathrm{Bo}=1.3 \times 10^{-4}, \mathrm{Oh}=2.0 \times 10^{-2},(\mathrm{~d})$ a water droplet and an ethanol droplet (no simulations available, see the discussion in section 8.2.3) with $\tilde{\gamma}=2.17, \mathrm{We}=11.80, \mathrm{Bo}=1.7 \times 10^{-4}, \mathrm{Oh}=1.9 \times 10^{-2}$, (e) a water droplet and a $34.5 \mathrm{wt} \%$ ethanol droplet with $\tilde{\gamma}=1.27$, We $=2.27$, $\mathrm{Bo}=1.5 \times 10^{-4}, \mathrm{Oh}=1.9 \times 10^{-2}$. 


\subsection{Collisions between droplets of different surface tensions}

because the formation of a toroidal bubble (which forms when the capillary waves coalesce with the opposing droplet) causes the simulation to fail [57].

\subsection{Collisions between droplets of different surface tensions}

We first discuss our results related to the collision between two droplets with different surface tensions at finite We. Figure 8.3 gives an overview of the collision dynamics observed for various combinations of $\tilde{\gamma}$ and We. A collision between two identical droplets $(\tilde{\gamma}=0)$ at low We is shown in Fig. 8.3a. The neck region (as defined in Fig. 8.2) rapidly grows upon first contact of the droplets due to the large curvature near the point of contact. This is accompanied by the formation of capillary waves, which travel over the droplets' interfaces and constructively interfere at the droplets' apexes, forming protrusions. The amplitude and propagation dynamics of the capillary waves on both droplets are equal, since $\gamma_{1}=\gamma_{2}$ [55]. The protrusions thus form in phase and with equal amplitudes on both droplets. As a result, the droplet shape remains symmetric during the entire coalescence process.

Figure 8.3b shows a collision between two identical droplets at high We. Symmetry is maintained, since $\tilde{\gamma}=0$. The shape of the droplet, however, is significantly different from the shape of the droplet in Fig. 8.3a-it is more elongated in the vertical direction, and the protrusions have a lower amplitude. The vertical elongation is caused by the higher inertia of the droplets, which, combined with incompressibility of the liquid, forces an outward vertical flow $[207,223]$. The protrusion amplitude is smaller due to the smaller time scale of the collision (28.5 $\mu$ s in Fig. 8.3a versus $22.0 \mu$ s in Fig. 8.3b). Since capillary waves grow over time, the amplitude of the capillary waves in a high We collision (which have a smaller time scale) remains smaller than the amplitude of capillary waves in low We collisions (larger time scale) [55]. Hence, the protrusion amplitude in Fig. 8.3b is smaller than that in Fig. 8.3a.

We now turn to collisions with $\tilde{\gamma}>0$, as shown in Fig. 8.3c-e. Figure 8.3c shows a collision with high $\tilde{\gamma}$ at low We. A striking difference with respect to the collisions with $\tilde{\gamma}=0$ is observed. Namely, the coalescing droplets take on a highly asymmetric shape. A similar asymmetric shape was reported by Gao et al. and Kohno et al. [211-213]. Here, a slender protrusion with large amplitude forms at the side of the water droplet, whereas a small protrusion forms on the ethanol droplet. The difference in protrusion size is caused by the different surface tensions of the droplets. Similarly, the different surface 
tensions result in different capillary wave velocities, such that the protrusions reach their maximum size out of phase [55]. The coalescence dynamics and capillary waves are further affected by the Marangoni effect, which causes ethanol to engulf the water droplet [63]. In some cases, the protrusion pinchesoff, such that a satellite droplet is formed, in a process known as 'partial coalescence' [216,224-227]. However, we typically observe that capillarity acts to pull the protrusion back in, before pinch-off occurs, to restore the droplet to a spherical shape. In section 8.4 we study capillary waves in the presence of the Marangoni effect in the limiting case of $\mathrm{We}=0$, i.e., in the absence of inertia.

The asymmetry that forms when $\tilde{\gamma}>0$ can be strongly reduced by increasing We, as shown in Fig. 8.3d. The protrusions in Fig. 8.3d are smaller than those in Fig. 8.3c, and are of similar size on both sides of the droplet. We note that, though the droplet shape is not mirror symmetric around the vertical axis, such as in Figs. 8.3a-b, its vertically elongated shape is similar to that of the droplet shown in Fig. 8.3b for $\tilde{\gamma}=0$. Key for the strong reduction of the asymmetric droplet shapes (which are caused by asymmetric surface tension) is that the inertia of both droplets is similar, i.e., $\rho_{1} v_{1, x}{ }^{2} R_{1} \approx \rho_{2} v_{2, x}{ }^{2} R_{2}$. When We $\gg 1$, the (symmetric) inertia dominates over the (asymmetric) surface tension, such that the droplet shape remains largely symmetric during coalescence. Finally, Fig. 8.3e shows a collision with moderate $\tilde{\gamma}$ and We, such that the contributions of inertia and surface tension are roughly equal. Indeed, the droplet shape is asymmetric, but less so than in Fig. 8.3c, reinforcing the importance of both $\tilde{\gamma}$ and We for the droplet shape.

We further quantify the effects of $\tilde{\gamma}$ and We on the asymmetry in Fig. 8.4, where we show the maximum asymmetry, which we quantify by the quantity $\max \left[L_{2}(t) / L_{1}(t)\right]$, as a function of $\mathrm{We}^{1 / 2}$ for several $\tilde{\gamma}$. Here, $L_{1}(t)$ and $L_{2}(t)$ are the time-dependent lengths from the apexes of the two droplets to the point of maximum vertical extension, as defined in the schematic in Fig. 8.4. Their ratio gives a measure for the asymmetry of the droplet shape. Figure 8.4 shows the maximum value that the ratio $L_{2}(t) / L_{1}(t)$ reaches during the collision. High asymmetry is observed for high $\tilde{\gamma}$, but can be strongly reduced by increasing We (see snapshots (i)-(iii) in Fig. 8.4). By contrast, droplets with $\tilde{\gamma}=0$ always remain symmetric (see snapshots (iv)-(vi)). In line with our observations in Fig. 8.3, we conclude that the asymmetry grows as $\tilde{\gamma}$ increases, and that it decreases with increasing We. Thus, the droplet shape is determined by the competition between the surface tension difference and the inertia of the colliding droplets. 


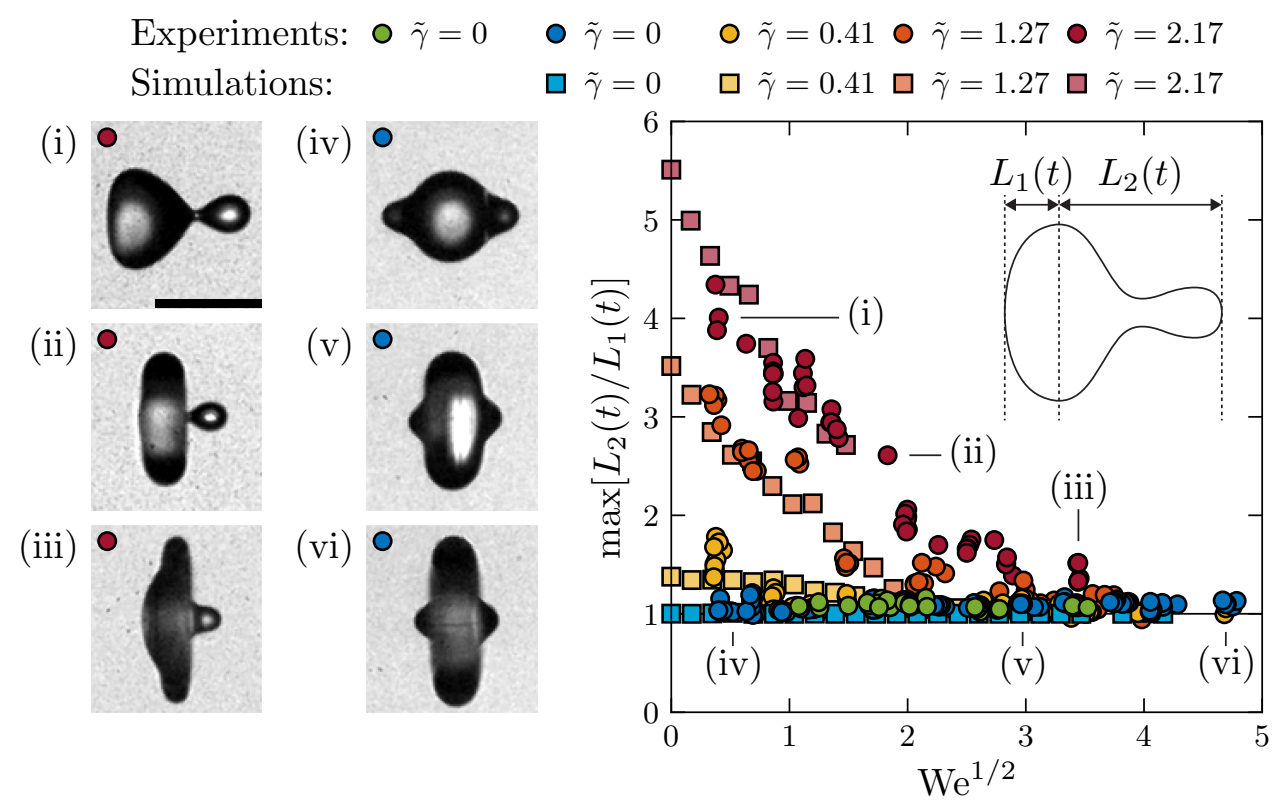

Figure 8.4: The maximum asymmetry $\max \left[L_{2}(t) / L_{1}(t)\right]$ as a function of $\mathrm{We}^{1 / 2}$ for several $\tilde{\gamma}$. The schematic inset shows the definitions of $L_{1}(t)$ and $L_{2}(t)$. In these experiments (circles) and simulations (squares) $\gamma_{1}$ was varied and $\gamma_{2}=72.5 \mathrm{mN} / \mathrm{m}$ was fixed, except for the green symbols, where $\gamma_{1}=\gamma_{2}=22.9 \mathrm{mN} / \mathrm{m}$. The snapshots (i) $-(\mathrm{vi})$ correspond to the indicated data points. The scale bar corresponds to $100 \mu \mathrm{m}$ and applies to all snapshots.

While our results show that asymmetric capillary waves are of paramount importance to the asymmetric droplet shape, the precise influence of the surface tension difference on the capillary waves remains to be determined. For that reason, we systematically study capillary waves in the presence of the Marangoni effect in the next section.

\subsection{Asymmetric capillary waves}

Asymmetric capillary waves form when two droplets of different surface tensions coalesce. In the previous section, we showed that this leads to asymmetric droplet shapes. Here, we focus solely on the dynamics of the capillary waves, which we study using numerical simulations of a model system. It will turn 
out that the capillary waves, and not the Marangoni effect, are responsible for the observed droplet asymmetry.

In the model system we impose that all properties of the droplets are equal, except their surface tensions. Specifically, we set $\rho=1000 \mathrm{~kg} / \mathrm{m}^{3}$, $\eta=1 \mathrm{mPa} \cdot \mathrm{s}, D=1 \times 10^{-9} \mathrm{~m}^{2} / \mathrm{s}, R=35 \mu \mathrm{m}$, and use a linear model for the surface tension $\gamma=\gamma_{2}+\left(\gamma_{1}-\gamma_{2}\right) c$, where $c$ varies between 0 and 1 , and $\gamma_{1} \geq 20 \mathrm{mN} / \mathrm{m}$ and $\gamma_{2} \leq 100 \mathrm{mN} / \mathrm{m}$. While this constitutes a simplified model, the values used here are close to those of a water-ethanol system, and the dimensionless groups are of the same order of magnitude for this model and the experiments presented before.

Figure 8.5 shows example snapshots of these simulations for various We. A thin film of the low surface tension liquid is observed to engulf the high surface tension droplet during the coalescence process, due to the Marangoni effect [63]. This induces tangential stresses at the interface which are expected to change the capillary wave dynamics and shape. For example, when a low surface tension droplet is deposited on a liquid substrate of higher surface tension, these stresses can induce a local interface distortion know as the 'Marangoni ridge' $[105,106]$.

In the remainder of this chapter, we further simplify the problem by excluding inertia, i.e., we set $\mathrm{We}=0$. Example snapshots of such simulations are shown in Fig. 8.6. The simulations reveal that the main morphological change of the droplet shape takes place on the droplet with high surface tension, where the capillary waves take on a different shape, depending on the value of $\tilde{\gamma}$. By contrast, the low surface tension droplet (with $\gamma_{1}=20 \mathrm{mN} / \mathrm{m}$ fixed for the simulations shown in Fig. 8.6) appears to be relatively unaffected by changes in $\tilde{\gamma}$. Additionally, we note that, despite their miscibility, the two liquids do not strongly mix during the coalescence process, in line with previous observations for a similar system [213].

To better understand the influence of $\tilde{\gamma}$ on the dynamics of the capillary waves, we study the shape of the capillary waves over time with respect to the original droplet shape at $t=0$. Figure 8.7 shows the method used to extract the capillary wave amplitude. A similar method was successfully used by Thoroddsen et al. for millimetre-sized drop coalescence [61]. Figure 8.8a shows the capillary waves that appear on coalescing droplets in the absence of a surface tension difference $(\tilde{\gamma}=0$, We $=0)$. In Fig. $8.8 \mathrm{~b}$, we attempt to collapse the capillary waves using the similarity scaling proposed by Keller \& Miksis for capillary waves on flat surfaces [55]. We do not find perfect collapse of the data, probably due to the high curvature of the droplet interface. 
composition

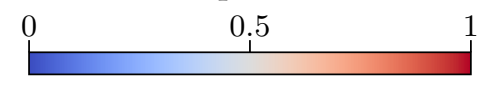

(a) $\mathrm{We}=0$

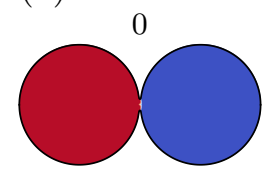

(b) $\mathrm{We}=1.75$

0

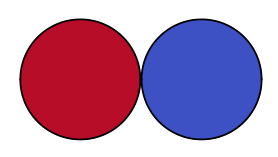

(c) $\mathrm{We}=10.93$

0

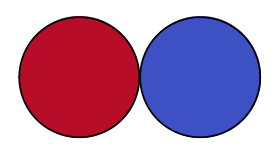

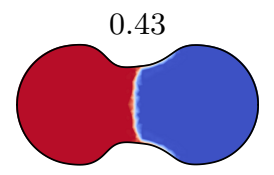

0.26

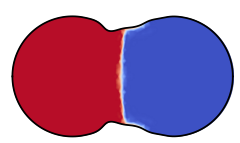

0.15

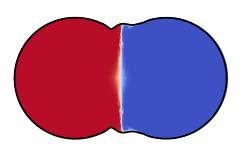

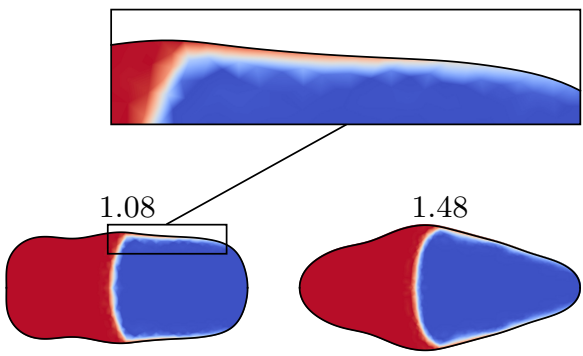
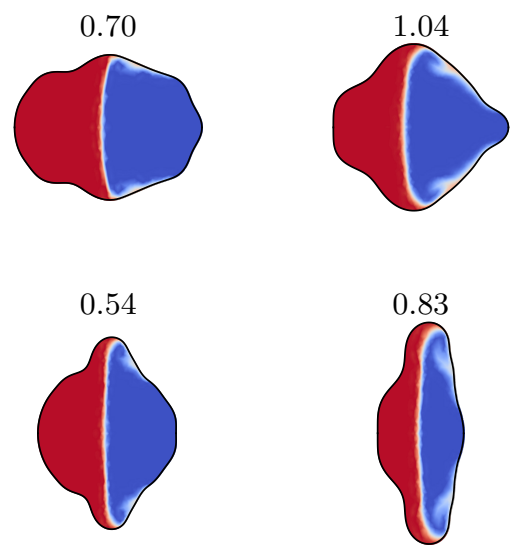

Figure 8.5: Snapshots from the numerical simulations with $\tilde{\gamma}=1$ and various We. Here, $\rho=1000 \mathrm{~kg} / \mathrm{m}^{3}, \eta=1 \mathrm{mPa} \cdot \mathrm{s}, \gamma_{1}=40 \mathrm{mN} / \mathrm{m}$, and $\gamma_{2}=80 \mathrm{mN} / \mathrm{m}$. The numbers above the snapshots indicate the nondimensional time $\tilde{t}=t / \tau_{2}$, with $\tilde{t}=0$ being the moment of first contact between the droplets. The zoom provides a closer look at the engulfing film. The protrusion amplitude (and the collision time scale) are smaller for higher We. 
Chapter 8. Asymmetric coalescence is caused by capillary waves

composition

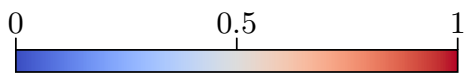

(a) $\tilde{\gamma}=0$
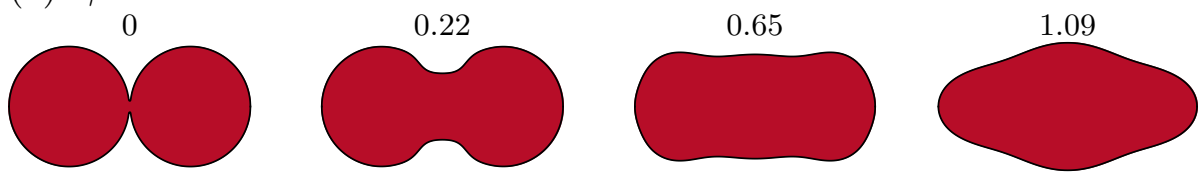

(b) $\tilde{\gamma}=0.5$

0

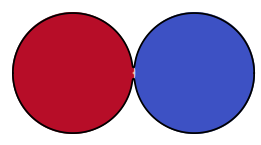

(c) $\tilde{\gamma}=1$
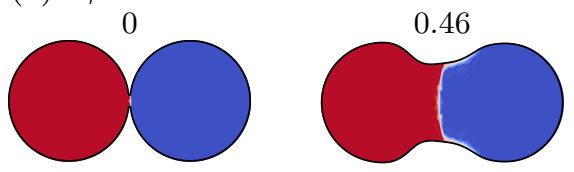

(d) $\tilde{\gamma}=2$
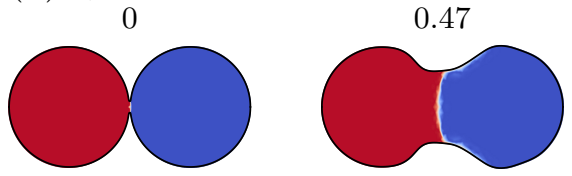

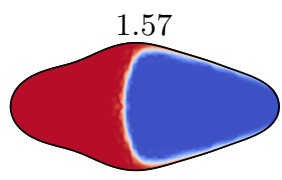

1.32

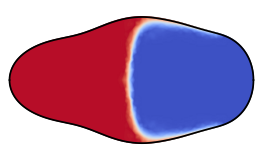

1.68

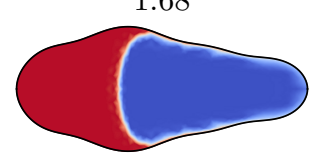

2.79

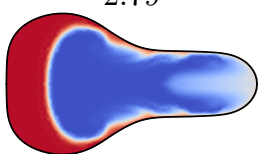

Figure 8.6: Snapshots from the numerical simulations with $\mathrm{We}=0$ and various $\tilde{\gamma}$. Here, $\rho=1000 \mathrm{~kg} / \mathrm{m}^{3}, \eta=1 \mathrm{mPa} \cdot \mathrm{s}, \gamma_{1}=20 \mathrm{mN} / \mathrm{m}$, and $\gamma_{2}$ is varied between $20 \mathrm{mN} / \mathrm{m}$ and $60 \mathrm{mN} / \mathrm{m}$, resulting in the shown values of $\tilde{\gamma}$. The numbers above the snapshots indicate $\tilde{t}=t / \tau_{2}$. The protrusion amplitude increases with increasing $\tilde{\gamma}$, resulting in highly asymmetric droplet shapes. 


\subsection{Asymmetric capillary waves}

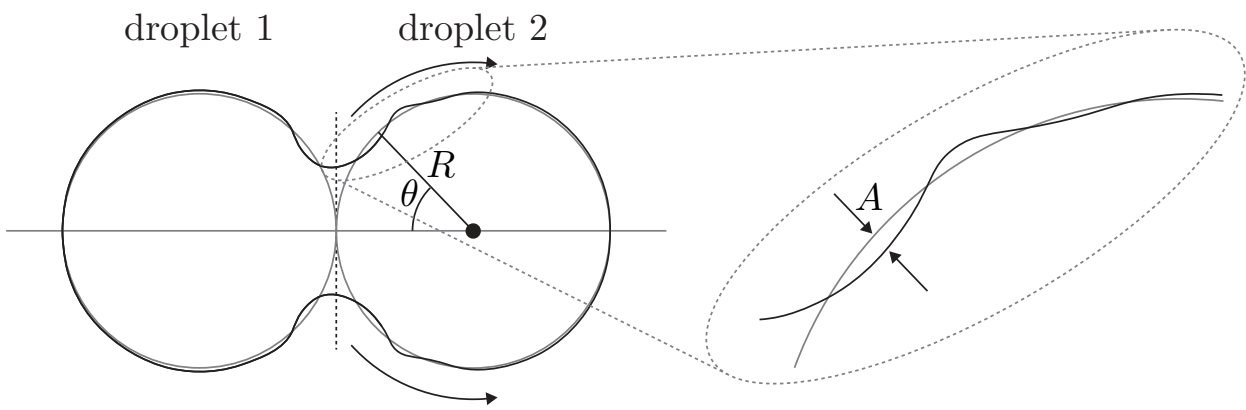

Figure 8.7: Schematic of the capillary waves on the droplets. The amplitude $A$ of the capillary waves is determined with respect to the shape of the droplet at $t=0$ as a function of $\theta$, which is the angle between an arbitrary point on the droplet interface and the centre of the droplet at $t=0$. In this case we neglect the small initial connection between the droplets, and assume that the droplets are perfect circles. The zoom shows the definition of $A$.

However, in Fig. 8.8c-d we report the location of the capillary wave maximum and the amplitude of the capillary waves as a function of time, finding that their dynamics are indeed close to the expected $t^{2 / 3}$ scaling [55].

We now return to coalescence with $\tilde{\gamma}>0$. The capillary waves that form on the droplets for several values of $\tilde{\gamma}$ are shown in Fig. 8.9. The amplitude of the capillary waves on the low surface tension droplet (droplet 1 ) is shown in Fig. 8.9a for two different non-dimensional times $\tilde{t}_{1}$ and $\tilde{t}_{2}$, where $\tilde{t}=t / \tau_{1}$ with $\tau_{1}=\left(\rho R^{3} / \gamma_{1}\right)^{1 / 2}$. The surface tension $\gamma_{1}=20 \mathrm{mN} / \mathrm{m}$ for the three cases shown in Fig. 8.9a, and remains constant during the coalescence process, since the Marangoni flow is directed from droplet 1 to droplet 2 . We observe that $\tilde{\gamma}$ has almost no effect on the capillary waves on droplet 1 - their amplitude and propagation dynamics are nearly the same for all $\tilde{\gamma}$.

Figure $8.9 \mathrm{~b}$ shows the corresponding capillary waves on droplet 2 at $\tilde{t}_{1}$ and $\tilde{t}_{2}$, where the time is now normalised by $\tau_{2}=\left(\rho R^{3} / \gamma_{2}\right)^{1 / 2}$. Several significant effects of $\tilde{\gamma}$ on the capillary waves on droplet 2 can be observed. First, the capillary waves are strongly deformed by the presence of the Marangoni effect. Compare, for example, the shape of the first local maximum (at $\theta \approx \pi / 2$ for $\tilde{t}_{2}$ ), which appears damped and distorted for $\tilde{\gamma}=1$. One would expect the amplitude of the capillary waves on droplet $2\left(A_{2}\right)$ to be larger than the amplitude of the capillary waves on droplet $1\left(A_{1}\right)$, since $\gamma_{2}>\gamma_{1}$, yet, when we compare $A_{1}$ (Fig. 8.9a) to $A_{2}$ (Fig. 8.9b), we find the inverse, at 
Chapter 8. Asymmetric coalescence is caused by capillary waves
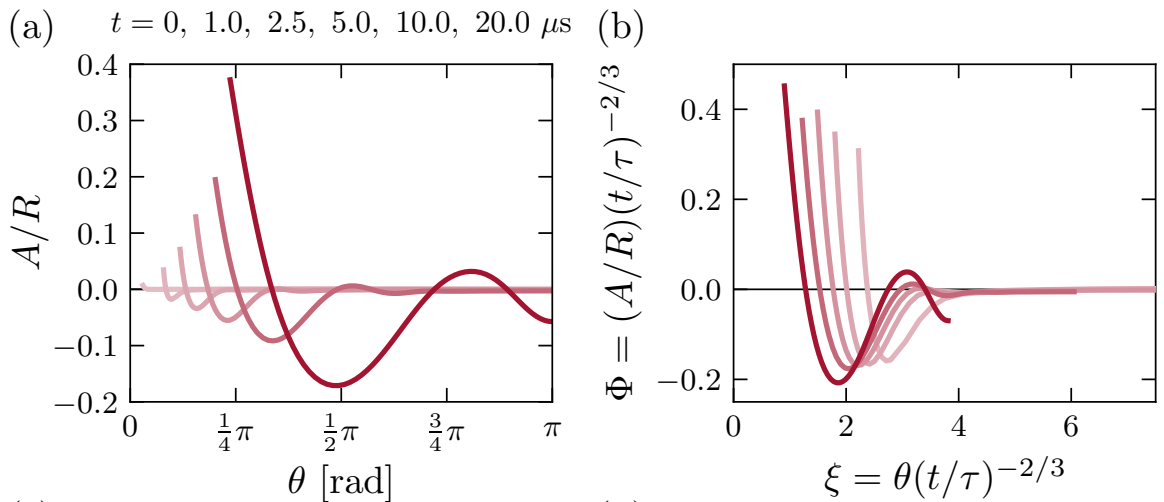

(c)
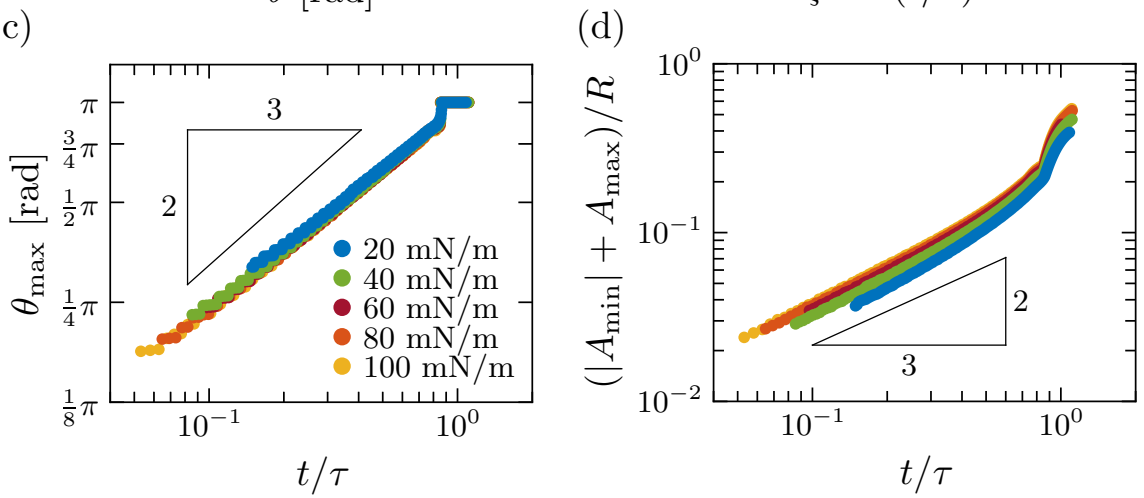

Figure 8.8: Capillary waves on coalescing droplets for $\tilde{\gamma}=0$ and $\mathrm{We}=0$, from our numerical simulations. (a) Capillary waves on a droplet with $\gamma=60 \mathrm{mN} / \mathrm{m}, A$ is the amplitude and $\theta$ the angular coordinate as defined in Fig. 8.7. (b) Similarity collapse of the capillary waves, following Ref. [55]. (c) The location of the capillary wave maximum as a function of time. (d) The amplitude of the capillary wave as a function of time. 
(a)

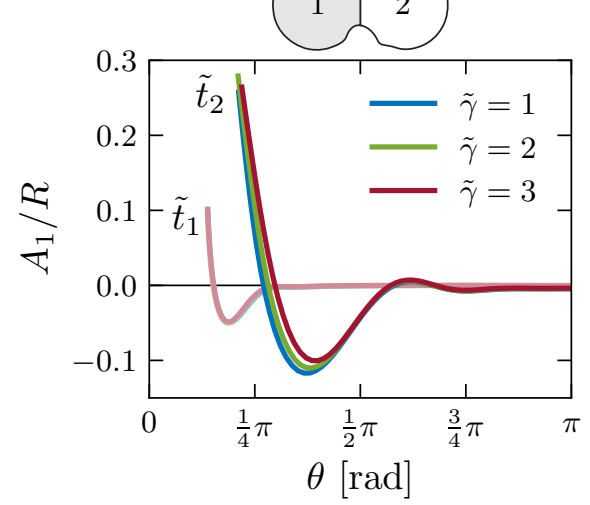

(b)

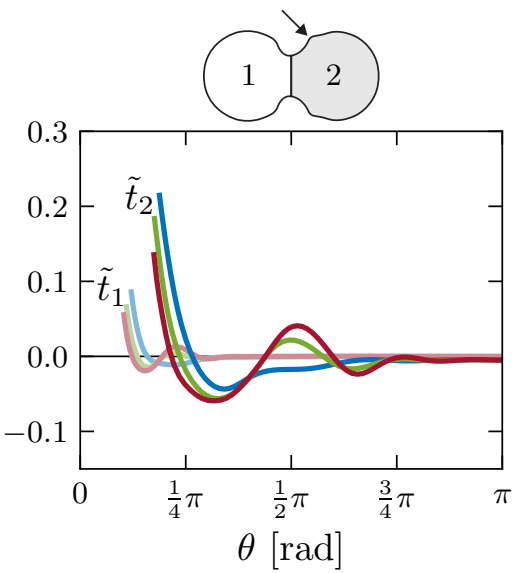

Figure 8.9: Capillary waves for various $\tilde{\gamma}$ at $\tilde{t}_{1}=0.15$ and $\tilde{t}_{2}=0.50$, from our numerical simulations. Here, $\gamma_{1}=20 \mathrm{mN} / \mathrm{m}$, and $\gamma_{2}=40,60,80 \mathrm{mN} / \mathrm{m}$, resulting in $\tilde{\gamma}=1,2,3$. (a) Capillary wave amplitude on the low surface tension droplet $\left(\tilde{t}=t / \tau_{1}\right)$. (b) Capillary wave amplitude on the high surface tension droplet $\left(\tilde{t}=t / \tau_{2}\right)$.
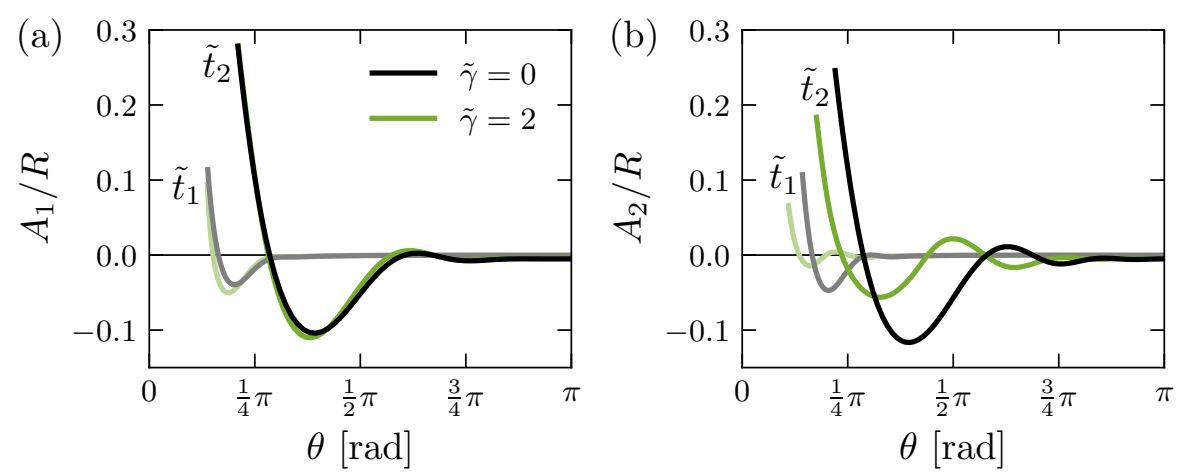

Figure 8.10: Capillary waves for $\tilde{\gamma}=0$ and $\tilde{\gamma}=2$ at $\tilde{t}_{1}=0.15$ and $\tilde{t}_{2}=0.50$, from our numerical simulations. (a) Capillary wave amplitude on the low surface tension droplet $\left(\tilde{t}=t / \tau_{1}\right)$, with $\gamma_{1}=20 \mathrm{mN} / \mathrm{m}$. The black line indicates capillary waves on a droplet with $\gamma=20 \mathrm{mN} / \mathrm{m}$. (b) Capillary wave amplitude on the high surface tension droplet $\left(\tilde{t}=t / \tau_{2}\right)$, with $\gamma_{2}=40 \mathrm{mN} / \mathrm{m}$. The black line indicates capillary waves on a droplet with $\gamma=40 \mathrm{mN} / \mathrm{m}$. 
least for the amplitude of the first local minimum. Second, the propagation of the capillary waves is slower for increasing $\tilde{\gamma}$. The higher $\tilde{\gamma}$ waves are consistently lagging behind the $\tilde{\gamma}=1$ wave in Fig. 8.9b. It is, however, important to note that $\gamma_{2}$ is not identical for the three cases considered in Fig. 8.9b. The capillary waves in Fig. $8.9 \mathrm{~b}$ are therefore not expected to collapse, since their properties depend on $\gamma_{2}$ [55]. We therefore compare the capillary waves for $\tilde{\gamma}=2$ to those for $\tilde{\gamma}=0$ in Fig. 8.10, to verify that the difference between the capillary waves in Fig. 8.9b is (at least partly) caused by $\tilde{\gamma} \neq 0$ and not solely by the difference in $\gamma_{2}$. In Fig. 8.10a we compare the wave amplitude $A_{1}$ for the two cases with $\tilde{\gamma}=0$ and $\tilde{\gamma}=2$, where for both cases $\gamma_{1}=20 \mathrm{mN} / \mathrm{m}$. In line with the results shown in Fig. 8.9a, there is no effect of $\tilde{\gamma}$ on the wave amplitude $A_{1}$. In Fig. $8.10 \mathrm{~b}$ we show a similar comparison for the capillary waves on droplet 2 , where we note that $\gamma_{2}=40 \mathrm{mN} / \mathrm{m}$ for both cases. Here, we once again observe that the damping increases and wave propagation decreases with increasing $\tilde{\gamma}$. We note that a similar damping effect on capillary waves has recently been observed for the coalescence of a surfactant-laden droplet coalescing with a liquid bath [228]. We thus conclude that $\tilde{\gamma}$ has a strong effect on the capillary waves (and by extent, the protrusion) on droplet 2. Additionally, the damping effect of $\tilde{\gamma}$ indicates that the Marangoni effect does not drive the asymmetric droplet shapes observed in Figs. 8.3 and 8.4, in contrast to the findings of Gao et al. and Kohno et al. [211-213]. In fact, the intrinsic dependence of the capillary wave dynamics on surface tension drives the asymmetry, and remarkably, the Marangoni effect decreases the asymmetry.

Figures $8.9 \mathrm{~b}$ and $8.10 \mathrm{~b}$ show that the capillary waves on the high surface tension droplet are strongly affected by the engulfing front of the low surface tension liquid. However, it is not yet clear if the distortion of the capillary wave is universal. In Fig. 8.11 we therefore compare the local surface tension to the capillary wave amplitude as a function of $\theta$ for two $\tilde{\gamma}$. The dashed line in Fig. 8.11 indicates the location of the engulfing front, which we define as the first $\theta$ where $\gamma<\gamma_{2}$. Figure 8.11 reveals that the location of the engulfing front with respect to the capillary wave is not universal, since the location of the front with respect to the capillary wave is different in Fig. 8.11a and b. This implies that the dynamics of the capillary waves (characterised by $\theta_{\max } \propto t^{2 / 3}$ ) are different than that of the engulfing front. To further quantify this point, we therefore track the front location with respect to the initial droplet position at $t=0$ in Fig. 8.12. Following Koldeweij et al., we rescale 
(a)

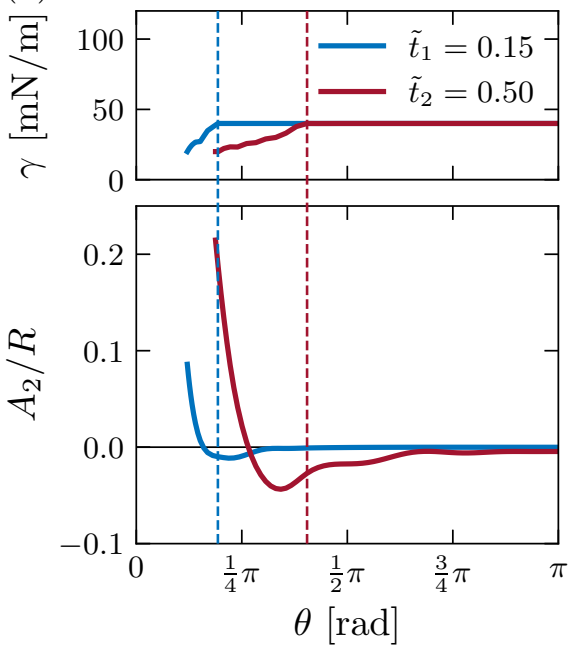

(b)

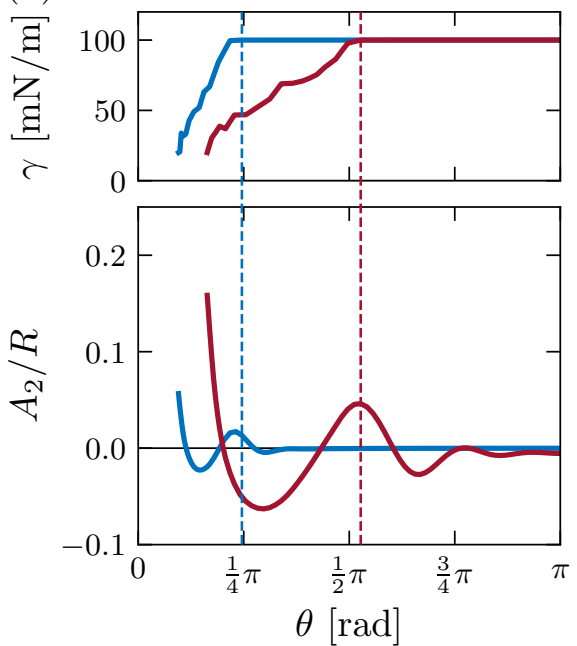

Figure 8.11: Surface tension and relative capillary wave amplitude on droplet 2 from our numerical simulations for (a) $\tilde{\gamma}=1$, and (b) $\tilde{\gamma}=4$. In both cases $\gamma_{1}=20 \mathrm{mN} / \mathrm{m}$. The vertical dashed lines indicate the engulfing front locations.

the time and front location using

$$
\hat{t}=\frac{t}{\eta^{3} / \Delta \gamma^{2} \rho}, \quad \hat{L}=\frac{\theta_{\text {front }} R}{\eta^{2} / \Delta \gamma \rho},
$$

where $\Delta \gamma=\gamma_{2}-\gamma_{1}[63,229]$. While the data collapses at large $\hat{t}$, deviations can be observed for small $\hat{t}$ due to the initial conditions of our simulations. We find that the dynamics converge to $\hat{L} \propto t^{3 / 4}$. The same exponent was found for ethanol spreading over millimetre-sized drops in the pendant geometry [63]. It follows from balancing the Marangoni forces and the viscous forces in the thin boundary layer of the spreading liquid [230-232]. We thus find that the engulfing front exhibits different dynamics $\left(\propto t^{3 / 4}\right)$ than the capillary wave $\left(\propto t^{2 / 3}\right)$, reflecting the different driving mechanisms. This explains why the shape of capillary waves in the presence of the Marangoni effect is non-universal. 
Chapter 8. Asymmetric coalescence is caused by capillary waves

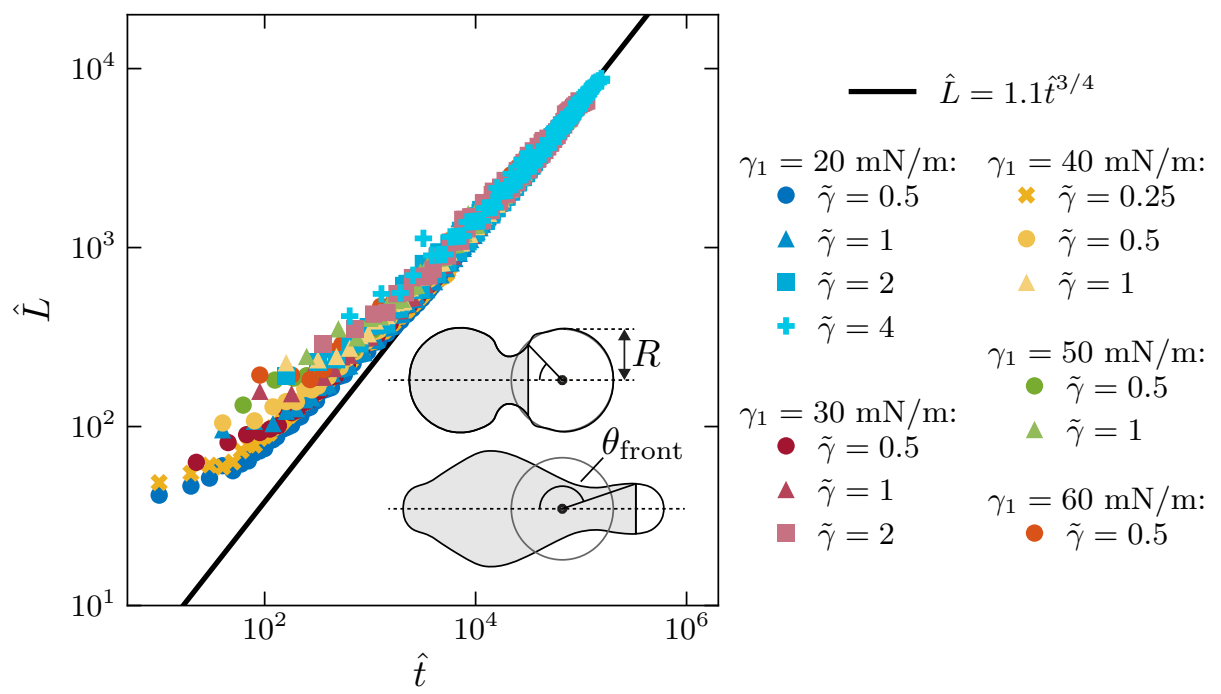

Figure 8.12: Rescaled location of the engulfing front as a function of rescaled time, from our numerical simulations. The schematic inset shows the definition of $\theta_{\text {front }}$, which is the angle between the location of the engulfing front on the droplet interface with respect to the centre of the initial droplet position at $t=0$. The data are cut off when $\theta_{\text {front }}=\pi$. The front dynamics converge to $\hat{L} \propto t^{3 / 4}$, in accordance with the results of Koldeweij et al. [63].

\subsection{Conclusion and outlook}

In this chapter, we have studied collisions between two droplets with different surface tensions. Using a combination of experiments and numerical simulations, we have shown that the shape of the droplets during coalescence can be highly asymmetric. By contrast, the shape of colliding droplets with identical surface tensions remains symmetric at all times. Furthermore, we have shown that the droplet shape is determined by a delicate competition between the surface tension difference and inertia - the asymmetry increases with increasing surface tension difference, and can be reduced by increasing the inertia of the colliding droplets.

Our results show that the interference of capillary waves is at the origin of the asymmetric droplet shapes. Since the amplitude and propagation dynamics of capillary waves depend on the surface tension of the interface that they travel on, the capillary waves are asymmetric in the coalescence of two droplets 


\subsection{Conclusion and outlook}

with different surface tensions. Contrary to previous works, we find that the Marangoni effect has a damping effect on the capillary waves [211-213]. Hence, we find that the asymmetry is primarily caused by the intrinsic difference between the capillary waves amplitude and propagation dynamics set by the initial surface tension of the droplets, and not by the Marangoni effect. In fact, the Marangoni effect decreases the asymmetry, by damping the capillary waves.

The results presented here show the richness of phenomena which can occur in physico-chemical hydrodynamics, and that these are often counterintuitive. From a more applied point of view, they may be of interest to microfluidic applications where coalescence of droplets with different surface tensions is prevalent, such as the in-air microfluidic fabrication of emulsions, colliding-droplet chemical microreactors, or in other applications of physicochemical hydrodynamics $[14,68,233]$. 
Chapter 8. Asymmetric coalescence is caused by capillary waves

\subsection{Appendix}

\subsubsection{Influence of evaporation (experiments)}

Evaporation during the experiment can affect the properties of the droplets. Importantly, the surface tension of liquid mixtures may change due to evaporation if one component is more volatile than the other. In our experiment liquid evaporates from the dispenser nozzle (in the time between droplet jetting), during flight, and during the coalescence process. Here, we attempt to quantify the volume of the droplet that is lost due to evaporation. We assume that the droplet consists of ethanol, such that the evaporation calculated here is the worst-case scenario, since ethanol is more volatile than water.

The radius of an evaporating spherical drop is given by

$$
R(t)=\left[R_{0}^{2}-\left(\frac{2 D M}{\rho \mathcal{R} T}\right)\left(p_{0}-p_{\infty}\right) t\right]^{1 / 2},
$$

where $R_{0}$ is the initial droplet radius, $D$ is the diffusivity, $M$ is the molecular weight, $\mathcal{R}$ is the gas constant, $T$ is the temperature, $p_{0}$ is the vapour pressure close to the droplet's surface, $p_{\infty}$ is the vapour pressure far away from the droplet, and $t$ is the time [234].

A droplet typically spends $2 \mathrm{~ms}$ in flight when jetted at the lowest velocity. For an ethanol droplet with $R_{0}=35 \mu \mathrm{m}$ at room temperature Eq. 8.9 predicts $0.8 \%$ volume loss during the experiment. The coalescence process of the droplet typically does not exceed $100 \mu \mathrm{s}$, such that the volume lost due to evaporation during the coalescence process is at least one order of magnitude smaller than the volume lost during flight.

As an example, we calculate the change in the surface tension of a droplet consisting of ethanol and water, with $c=0.345$. Out of all liquids used in our experiments, this composition (with surface tension $31.9 \mathrm{mN} / \mathrm{m}$ ) is the most sensitive to changes in the surface tension due evaporation. Assuming that $0.8 \%$ of the ethanol volume is lost due to evaporation, the final composition is $c=0.343$, with $\gamma=32.0 \mathrm{mN} / \mathrm{m}$. In reality, the change in surface tension will be even smaller, since we calculated volume lost due to evaporation based on pure ethanol, and not a mixture of ethanol and water (for which the evaporation rate will be lower).

Liquid also evaporates at the nozzle exit of the dispenser in the time between droplet jetting. We therefore dispense droplets continuously at 200 droplets per second, such that the liquid at the dispenser exit is refreshed every $5 \mathrm{~ms}$, minimising compositional changes in the process. Due to the similar 


\subsection{Appendix}

timescales, we expect that the compositional change due to evaporation at the dispenser exit (over $5 \mathrm{~ms}$ ) is comparable to that during flight (which takes approximately $2 \mathrm{~ms}$ ), such that the surface tension change will be minimal. Hence, we conclude that the effect of evaporation on the surface tension can be neglected in our experiments and numerical simulations.

\subsubsection{Time step and grid resolution (numerical simulations)}
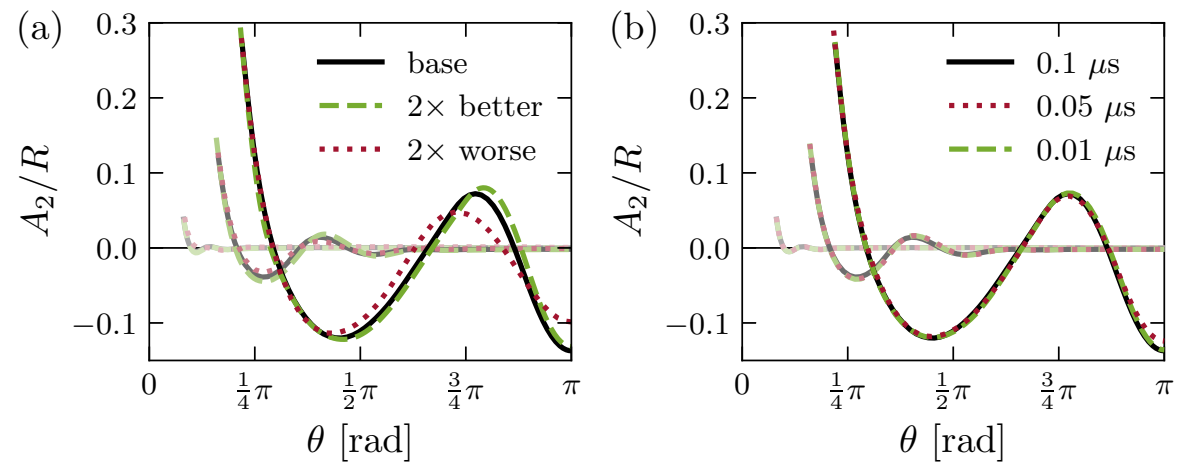

Figure 8.13: (a) Comparison of different grid resolutions. The black line shows the resolution used for the numerical simulations in the main text. (b) Comparison of different time steps. The black line shows the time step used for the numerical simulations in the main text. 



\section{Chapter 9}

\section{Conclusion and outlook}

In this thesis, we have studied the wetting properties (part I; chapters 24) and coalescence dynamics (part II; chapters 5-8) of drops in multi-phase flow systems. In particular, we have focused on the role of liquid substrates and multi-component drops. Beyond the discoveries that were presented in each chapter, this thesis as a whole contributes to the contemporary field of multi-phase capillary flows. Within this rich area of research, we specifically contribute to the following:

i. Liquid substrates can introduce new, complex, wetting phenomena due to their ability to transport matter by flow (as evidenced by chapters 2 and 3). Additionally, they provide an excellent way to study the importance of boundary conditions with respect to their solid substrate counterpart in capillary flow systems (chapters 5 and 6 ).

ii. Multi-component capillary flows can exhibit vastly different dynamics than single-component flows, even if one of the components constitutes only a small part of the whole (chapters 4 and 7). Furthermore, physicochemical processes and interaction between separate components play an important role in wetting and coalescence flows (chapters 3 and 8).

The research presented in this thesis was largely inspired by challenges associated with flows encountered in inkjet printing. Our findings could, in turn, serve as inspiration for inkjet printing developers. The multi-phase nature of inkjet printing often leads to problematic phenomena that lower the print quality. Several examples are shown in section 1.3; Fig. 1.10. We have used model systems to study these phenomena on a fundamental level, and provided plausible explanations for their origin, which may aid the further improvement 
of inkjet printing technology. In the following, we elaborate on the above two statements and propose several areas that might be of interest for future research, in particular in the context of inkjet printing.

\subsection{Wetting}

The first part of this thesis concerned the wetting properties of drops in multiphase systems. We broadly defined wetting as the behaviour of (multiple) drops on a variety of substrates. In chapter 2 , we revealed that liquid substrates can facilitate non-monotonic interaction between neighbouring drops by drop-induced surface deformations of the substrate. Then, in chapter 3 , we discovered that the combined effects of particle destabilisation and Marangoni flow can induce complex pattern formation dynamics.

Liquid substrates play a crucial role in both of these chapters - their deformation drives drop and particle motion, and (in chapter 3) it is used as a catalyst for local particle destabilisation. They also pose a major challenge, since flow in the substrate can affect the overall dynamics of the system. In chapter 2, for example, flow in the substrate affects the motion of the interacting drops. By contrast, such flow is absent in elastic substrates, for which a similar interaction between drops was observed $[71,72]$. In the latter case, the interacting drops are in a quasi-equilibrium state, which considerably simplifies the problem. In chapter 3 , the flow structure in the substrate underneath the drop during the initial spreading phase remains undetermined, though it is expected to affect the final pattern.

The flow in and around drops moving on liquid substrates thus introduces a new type of complexity. This is illustrated in Fig. 9.1, which reveals that the velocity of a drop moving on a liquid substrate depends on the history of the drop. Two experiments are shown. In the first experiment (blue data points), a drop is deposited on an inclined liquid substrate, upon which it starts moving under the influence of gravity. The velocity quickly reaches a transient peak and subsequently decreases to an equilibrium value. In the second experiment (red data points), a drop is deposited on a horizontal liquid substrate and left stationary for 30 minutes. The substrate is then inclined and the drop starts moving. In this case, the temporal evolution of the drop velocity is completely different - the velocity slowly increases over time, eventually reaching the same equilibrium value as in the first experiment. No transient peak is observed. The different outcomes of these experiments reveal that the motion of a drop on a liquid substrate depends on its history. It is expected that the velocity 


\subsection{Wetting}

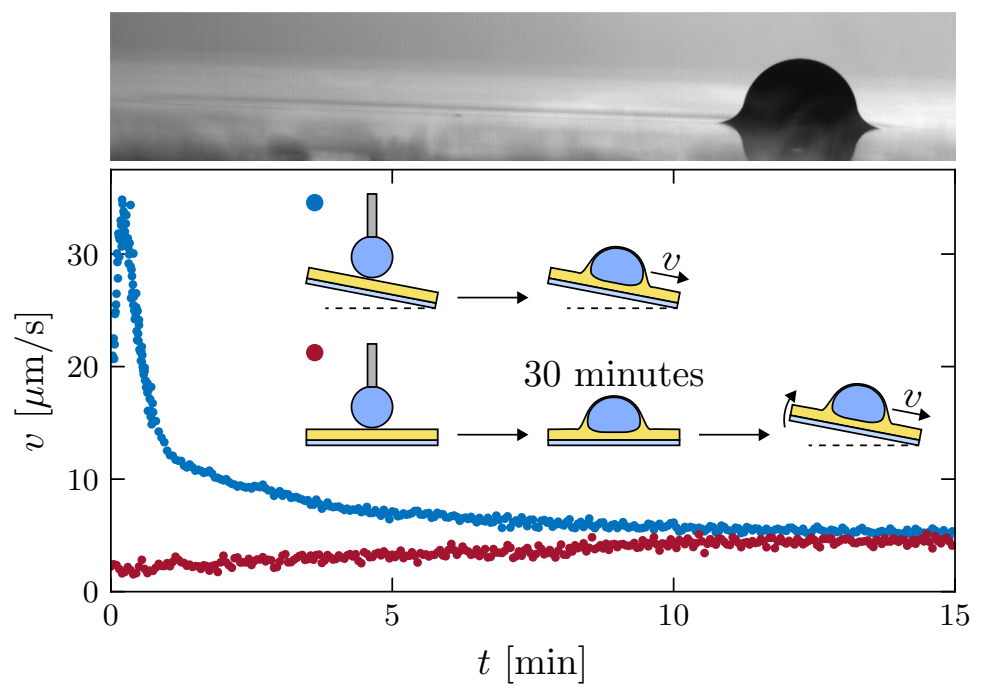

Figure 9.1: The velocity $v$ of a water drop (radius $\approx 1 \mathrm{~mm}$ ) sliding on a thin liquid substrate (thickness $\approx 28 \mu \mathrm{m}$, viscosity $\approx 1 \mathrm{~Pa} \cdot \mathrm{s}$ ) obtained from two different experiments. In one experiment, the drop is deposited on a liquid substrate inclined at an angle $\approx 2.4^{\circ}$ (blue). In the other experiment (red), the liquid substrate is left horizontal for 30 minutes before being inclined. The temporal evolution of the velocity depends on the history of the drop, though in both experiments the drop reaches the same steady-state velocity.

depends on the thickness of the film underneath the drop, which is smaller at the start of the second experiment due to drainage that occurs during the 30 minute waiting period [235]. Additional experiments that measure the dynamical evolution of the film morphology underneath the drop and close to the wetting ridge are needed to confirm this hypothesis, and to find the origin of the transient peak. This example illustrates that the motion of drops on liquid substrates contains rich unexplored physics, and offers an avenue for further research.

In chapter 4, we studied the wetting properties of two-component drops (on a solid substrate) and discovered that two mechanisms of completely different origin determine the contact angle. The only feature that distinguishes between these mechanisms is the flow structure inside the drop. To further extend our understanding of these systems it will be of interest to study the transition from one wetting state (Marangoni contraction) to the other wet- 
ting state (autophobing) with increasing concentration of vicinal alkanediol. When does this transition occur? This question might be answered by considering other surfactant-like liquids, with liquid properties (i.e., viscosity, surface tension, and volatility) that differ from those of vicinal alkanediols. Systematically changing these properties allows tuning of the strength of either mechanism, which should provide insight into the transitory regime. Another method to study the transition involves modifying the liquid properties of the drop by addition of a third component, such as a viscosity modifier (e.g., glycerol). Care needs to be taken with the latter method, as chapter 4 and other works (see, e.g., the perspective by Lohse \& Zhang, Ref. [14]) show that the properties of multi-component drops are often difficult to predict.

A comprehensive understanding of the physico-chemical hydrodynamics in multi-component drops will allow tailoring of the system according to the needs of technological applications, where some drop properties (such as the high mobility of Marangoni-contracted drops) can be (un)desired. This will be of particular interest to ink developers, since the desired properties of ink can be dissimilar at different stages of the printing process. For example, low wettability is desired to prevent wetting of the nozzle plate, but high wettability is required to form a uniform coating on the substrate. The dual wetting nature of aqueous mixtures of vicinal alkanediols may provide solutions to such problems, especially when the substrate properties (porous or impermeable, strong or weak affinity for autophobing molecules) are also considered.

\subsection{Coalescence}

The second part of this thesis concerned coalescence - the merging of two liquid bodies into one. In chapters 5 and 6 , we studied the self-similar coalescence dynamics of liquid lenses. In contrast to chapters 2 and 3 , the liquid substrate has a sub-dominant role here, which allows use of the thin-sheet equations. This set of equations provides an excellent description of the coalescence dynamics, in both the viscous and inertial regimes. The success of the thin-sheet equations, in particular in the inertial regime, makes the liquid lens coalescence geometry an interesting framework for more complex coalescence studies. More generally, this framework can be used to study to a wide variety of thinfilm flow problems. In particular, the thin-sheet equations (Eqs. 5.1-5.2) in conjunction with the thin-film equation (Eq. 1.2) allow for a detailed study of the importance of boundary conditions - both equations provide descriptions of flow in slender geometries, but each with different boundary conditions. 
(a)

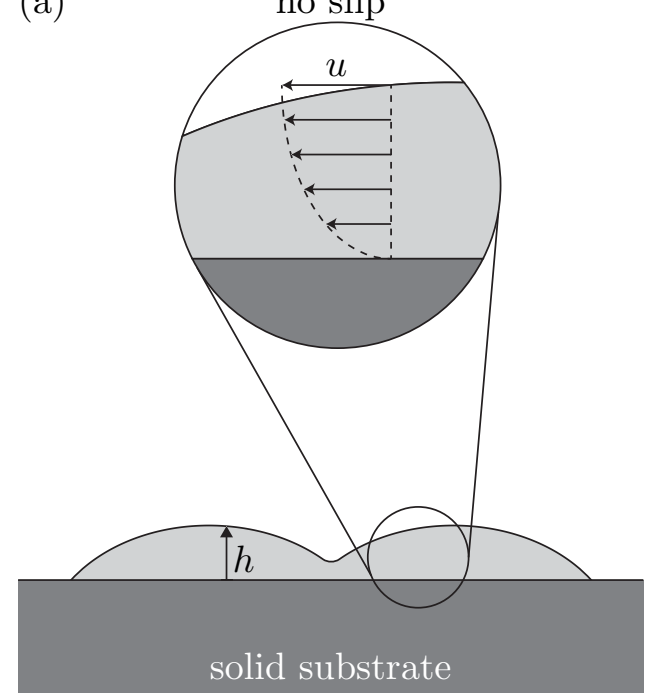

(b)

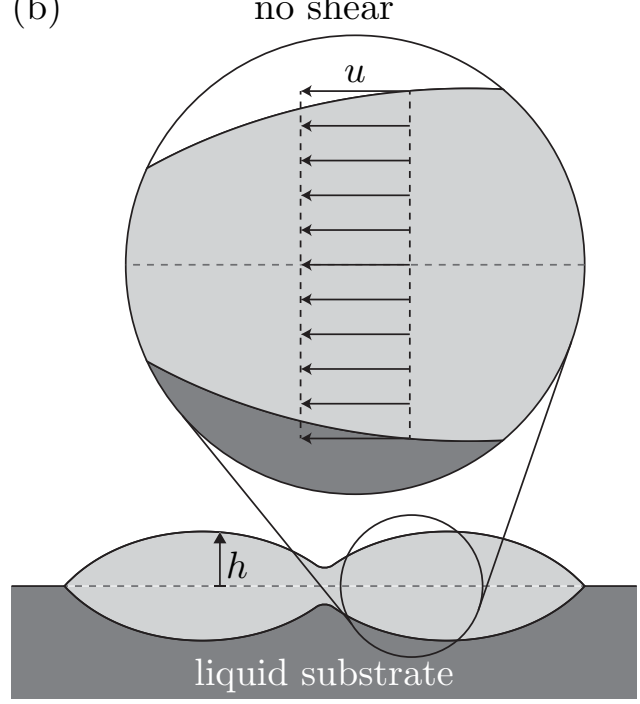

Figure 9.2: The coalescence of two drops on two different substrates. (a) Coalescence on a solid substrate involves a no-slip boundary condition at the solid-liquid interface, such that the velocity profile is parabolic, and the dynamics are described by the thin-film equation (Eq. 1.2). (b) Coalescence on a liquid substrate involves a no-shear boundary condition at the liquid-liquid interface, resulting in plug flow. The coalescence dynamics are described by the thin-sheet equation (Eqs. 5.1-5.2).

The thin-sheet equations apply to flows involving no-shear boundary conditions (Fig. 9.2a), whereas the thin-film equation describes flows with a no-slip boundary condition (Fig. 9.2b). Our work on liquid substrates, in combination with previous work on coalescence on solid substrates [44], shows that the sessile drop coalescence geometry provides an attractive way to study these two flow types both experimentally and theoretically.

This framework can be extended to study more complex multi-phase flows, such as the flow of surfactant-containing liquids or viscoelastic liquids, and flows that are affected by certain exterior influences, such as electric fields and temperature gradients. Such an extension involves the addition of additional fields (for example, the local surfactant concentration when considering the effect of surfactants) and transport equations to the governing equations to describe the evolution of these fields. The thin-film equation has been 
successfully extended using this method, and similar extensions exist for the thin-sheet equations $[170,236,237]$. A comparison of both flow geometries would provide an increased understanding of the influence of boundary conditions on complex flows. This will be of particular interest to inkjet printing developers, since ink is a complex liquid consisting of multiple components. Furthermore, the coalescence of ink drops occurs on both solid (paper) and liquid (primer layer) substrates. For the latter case it would be useful to study coalescence on relatively thin liquid substrates (as opposed to the deep pool considered in chapters 5 and 6 ), since the thickness of the primer layer is typically comparable to the ink drop size.

In chapters 7 and 8, we investigated the coalescence dynamics of multicomponent drops. First, we studied the coalescence of viscoelastic drops, in chapter 7 . We found that the temporal evolution of the bridge growth is relatively unaffected by the viscoelastic properties of the drops. By contrast, the spatial structure of the bridge is dramatically altered by the polymers. It will be of interest to study viscoelastic flows in the presence of other singularities as well: even though the singularity that is present at the start of coalescence is (to a large degree) similar to the singularity that occurs during pinch-off, the viscoelastic properties of the liquid manifest themselves in a completely different way. Other singular geometries (e.g., the moving contact line singularity reviewed in Ref. [17]) may reveal even more responses to the excitation of viscoelastic liquids.

Finally, in chapter 8, we studied the coalescence of two drops with different surface tensions. We showed that the shape of such drops during coalescence is determined by a balance between the surface tension difference and inertia, and that it can be highly asymmetric. Surprisingly, we discovered that the Marangoni effect has a damping effect on the capillary waves, and that it reduces the asymmetric shape of the drops during coalescence. This behaviour is different from that observed for the coalescence of drops with different surface tensions on solid substrates, where coalescence is 'delayed' by the Marangoni effect (Fig. 1.9b) [46,49, 52,53,64]. This highlights, once more, the importance of geometry and boundary conditions in capillary flows. 


\section{Bibliography}

[1] T. W. Secomb, "Blood flow in the microcirculation", Annu. Rev. Fluid Mech. 49, 443-461 (2017).

[2] J. C. Wyngaard, "Atmospheric turbulence", Annu. Rev. Fluid Mech. 24, 205-233 (1992).

[3] Arend, Wikimedia Commons, URL https://commons.wikimedia. org/wiki/File:Schaatsenrijder_-_water_strider_(44405792011) .jpg, CC BY 2.0.

[4] Issempa, Wikimedia Commons, URL https://commons.wikimedia. org/wiki/File:Lotus-Effekt_(Droplet_on_Leaf)_\%C2\%A9_W. _Barthlott,_Lotus-Salvinia.de.jpg, CC BY-SA 4.0.

[5] J. C. Loudet and B. Pouligny, "How do mosquito eggs self-assemble on the water surface?", Eur. Phys. J. E 34 (2011).

[6] K. G. Winkels, I. R. Peters, F. Evangelista, M. Riepen, A. Daerr, L. Limat, and J. H. Snoeijer, "Receding contact lines: from sliding drops to immersion lithography", Eur. Phys. J. Special Topics 192, 195-205 (2011).

[7] H. Wijshoff, "The dynamics of the piezo inkjet printhead operation", Phys. Rep. 491, 77-177 (2010).

[8] H. Wijshoff, "Drop dynamics in the inkjet printing process", Curr. Opin. Colloid Interface Sci. 36, 20-27 (2018).

[9] D. Lohse, "Fundamental fluid dynamics challenges in inkjet printing", Annu. Rev. Fluid Mech. (2022), in press.

[10] F. Varela, E. Armendáriz, and C. Wolluschek, "Inkjet printed electronics: the wet on wet approach", Chem. Eng. Process. 50, 589-591 (2011). 
[11] A. Fraters, R. Jeurissen, M. van den Berg, H. Reinten, H. Wijshoff, D. Lohse, M. Versluis, and T. Segers, "Secondary tail formation and breakup in piezoacoustic inkjet printing: femtoliter droplets captured in flight", Phys. Rev. Appl. 13, 024075 (2020).

[12] R. T. van Gaalen, C. Diddens, D. P. Siregar, H. M. A. Wijshoff, and J. G. M. Kuerten, "Absorption of surfactant-laden droplets into porous media: A numerical study", J. Colloid Interface Sci. 597, 149-159 (2021).

[13] M. A. Mulla, H. N. Yow, H. Zhang, O. J. Cayre, and S. Biggs, "Colloid particles in ink formulations", in Fundamentals of Inkjet Printing: The Science of Inkjet and Droplets, edited by S. D. Hoath, 141-168 (WileyVCH, Weinheim, Germany) (2016).

[14] D. Lohse and X. Zhang, "Physicochemical hydrodynamics of droplets out of equilibrium", Nat. Rev. Phys. 2, 426-443 (2020).

[15] D. Cressey, "365 days: Images of the year", Nature 516, 304-309 (2014).

[16] T. Young, "An essay on the cohesion of fluids", Philos. Trans. Soc. London 95, 65-87 (1805).

[17] J. H. Snoeijer and B. Andreotti, "Moving contact lines: Scales, regimes, and dynamical transitions", Annu. Rev. Fluid Mech. 45, 269-292 (2013).

[18] B. Andreotti and J. H. Snoeijer, "Statics and dynamics of soft wetting", Annu. Rev. Fluid Mech. 52, 285-308 (2020).

[19] S. Karpitschka, F. Liebig, and H. Riegler, "Marangoni contraction of evaporating sessile droplets of binary mixtures", Langmuir 33, 46824687 (2017).

[20] H. J. J. Staat, A. van der Bos, M. van den Berg, H. Reinten, H. Wijshoff, M. Versluis, and D. Lohse, "Ultrafast imaging method to measure surface tension and viscosity of inkjet-printed droplets in flight", Exp. Fluids 59, 2 (2017).

[21] G. Durey, H. Kwon, Q. Magdelaine, M. Casiulis, J. Mazet, L. Keiser, H. Bense, P. Colinet, J. Bico, and E. Reyssat, "Marangoni bursting: Evaporation-induced emulsification of a two-component droplet", Phys. Rev. Fluids 3, 100501 (2018). 


\section{Bibliography}

[22] A. Hamraoui, M. Cachile, C. Poulard, and A. Cazabat, "Fingering phenomena during spreading of surfactant solutions", Colloids Surf. 250, $215-221$ (2004).

[23] F. Wodlei, J. Sebilleau, J. Magnaudet, and V. Pimienta, "Marangonidriven flower-like patterning of an evaporating drop spreading on a liquid substrate", Nat. Commun. 9, 820 (2018).

[24] Y. Li, P. Lv, C. Diddens, H. Tan, H. Wijshoff, M. Versluis, and D. Lohse, "Evaporation-triggered segregation of sessile binary droplets", Phys. Rev. Lett. 120, 224501 (2018).

[25] L. Keiser, H. Bense, P. Colinet, J. Bico, and E. Reyssat, "Marangoni bursting: Evaporation-induced emulsification of binary mixtures on a liquid layer", Phys. Rev. Lett. 118, 074504 (2017).

[26] J. de Jong, H. Reinten, H. Wijshoff, M. van den Berg, K. Delescen, R. van Dongen, F. Mugele, M. Versluis, and D. Lohse, "Marangoni flow on an inkjet nozzle plate", Appl. Phys. Lett. 91, 204102 (2007).

[27] M. Jehannin, S. Charton, S. Karpitschka, T. Zemb, H. Möhwald, and H. Riegler, "Periodic precipitation patterns during coalescence of reacting sessile droplets", Langmuir 31, 11484-11490 (2015).

[28] R. D. Deegan, O. Baakajin, T. F. Dupont, G. Huber, S. R. Nagel, and T. A. Witten, "Capillary flow as the cause of ring stains from dried liquid drops", Nature 389, 827-829 (1997).

[29] H. Kim, F. Boulogne, E. Um, I. Jacobi, E. Button, and H. A. Stone, "Controlled uniform coating from the interplay of Marangoni flows and surface-adsorbed macromolecules", Phys. Rev. Lett. 116, 124501 (2016).

[30] Q. Xie and J. Harting, "From dot to ring: The role of friction in the deposition pattern of a drying colloidal suspension droplet", Langmuir 34, 5303-5311 (2018).

[31] X. Yang, V. H. Chhasatia, J. Shah, and Y. Sun, "Coalescence, evaporation and particle deposition of consecutively printed colloidal drops", Soft Matter 8, 9205-9213 (2012).

[32] Y. Li, C. Diddens, T. Segers, H. Wijshoff, M. Versluis, and D. Lohse, "Evaporating droplets on oil-wetted surfaces: Suppression of the coffeestain effect", Proc. Natl. Acad. Sci. U.S.A. 117, 16756-16763 (2020). 
[33] A. Gao, J. Liu, L. Ye, C. Schönecker, M. Kappl, H.-J. Bütt, and W. Steffen, "Control of droplet evaporation on oil-coated surfaces for the synthesis of asymmetric supraparticles", Langmuir 35, 14042-14048 (2019).

[34] A. Oko, A. Swerin, B. D. Brandner, D. Bugner, W. Cook, and P. M. Claesson, "Aggregation of inkjet ink components by $\mathrm{Ca}$ and $\mathrm{Mg}$ ions in relation to colorant pigment distribution paper", Colloids Surf. A 456, 92-99 (2014).

[35] J. C. Burton and P. Taborek, "Role of dimensionality and axisymmetry in fluid pinch-off and coalescence", Phys. Rev. Lett. 98, 224502 (2007).

[36] J. Eggers and E. Villermaux, "Physics of liquid jets", Rep. Prog. Phys. 71, 036601 (2008).

[37] J. Eggers, "Theory of drop formation", Phys. Fluids 7, 941-953 (1995).

[38] A. Deblais, K. P. Velikov, and D. Bonn, "Pearling instabilities of a viscoelastic thread", Phys. Rev. Lett. 120, 194501 (2018).

[39] C. Clasen, J. Eggers, M. A. Fontelos, J. Li, and G. H. McKinley, "The beads-on-string structure of viscoelastic threads", J. Fluid Mech. 556, 283-308 (2006).

[40] J. Eggers, M. A. Herrada, and J. H. Snoeijer, "Self-similar breakup of polymeric threads as described by the Oldroyd-B model", J. Fluid Mech. 887, A19 (2020).

[41] J. R. Castrejón-Pita, A. A. Castrejón-Pita, E. J. Hinch, J. R. Lister, and I. M. Hutchings, "Self-similar breakup of near-inviscid liquids", Phys. Rev. E. 86, 015301(R) (2012).

[42] W. D. Ristenpart, P. M. McCalla, R. V. Roy, and H. A. Stone, "Coalescence of spreading droplets on a wettable substrate", Phys. Rev. Lett. 97, 064501 (2006).

[43] J. D. Paulsen, R. Carmigniani, A. Kannan, J. C. Burton, and S. R. Nagel, "Coalescence of bubbles and drops in an outer fluid", Nat. Commun. 5, $3182(2014)$.

[44] J. F. Hernández-Sánchez, L. A. Lubbers, A. Eddi, and J. H. Snoeijer, "Symmetric and asymmetric coalescence of drops on a substrate", Phys. Rev. Lett. 109, 184502 (2012). 


\section{Bibliography}

[45] J. D. Paulsen, J. C. Burton, and S. R. Nagel, "Viscous to inertial crossover in liquid drop coalescence", Phys. Rev. Lett. 106, 114501 (2011).

[46] R. Borcia, S. Menzel, M. Bestehorn, S. Karpitschka, and H. Riegler, "Delayed coalescence of droplets with miscible liquids: Lubrication and phase field theories", Eur. Phys. J. E 34, 24 (2011).

[47] A. B. Thompson and J. Billingham, "Inviscid coalescence in the presence of a surrounding fluid", IMA J. Appl. Math. 77, 678-696 (2012).

[48] M. W. Lee, D. K. Kang, S. S. Yoon, and A. L. Yarin, "Coalescence of two drops on partially wettable substrates", Langmuir 28, 3791-3798 (2012).

[49] S. Karpitschka and H. Riegler, "Noncoalescence of sessile drops from different but miscible liquids: Hydrodynamic analysis of the twin drop contour as a self-stabilizing traveling wave", Phys. Rev. Lett. 109, 066103 (2012).

[50] A. Eddi, K. G. Winkels, and J. H. Snoeijer, "Influence of droplet geometry on the coalescence of low viscosity drops", Phys. Rev. Lett. 111, 144502 (2013).

[51] S. Karpitschka and H. Riegler, "Partial coalescence of sessile drops with different miscible liquids", Langmuir 29, 4426-4429 (2013).

[52] S. Karpitschka, C. Hanske, A. Fery, and H. Riegler, "Coalescence and noncoalescence of sessile drops: Impact of surface forces", Langmuir 30, 6826-6830 (2014).

[53] M. A. Bruning, M. Costalonga, S. Karpitschka, and J. H. Snoeijer, "Delayed coalescence of surfactant containing sessile droplets", Phys. Rev. Fluids 3, 073605 (2018).

[54] X. Xia, C. He, and P. Zhang, "Universality in the viscous-to-inertial coalescence of liquid droplets", Proc. Natl. Acad. Sci. U.S.A. 116, 2346723472 (2019).

[55] J. B. Keller and M. J. Miksis, "Surface tension driven flows", SIAM J. Appl. Math. 43, 268-277 (1983). 
[56] J. Billingham and A. C. King, "Surface-tension-driven flow outside a slender wedge with an application to the inviscid coalescence of drops", J. Fluid Mech. 533, 193-221 (2005).

[57] J. Eggers, J. R. Lister, and H. A. Stone, "Coalescence of liquid drops", J. Fluid Mech. 401, 293-310 (1999).

[58] D. G. A. L. Aarts, H. N. W. Lekkerkerker, H. Guo, G. H. Wegdam, and D. Bonn, "Hydrodynamics of droplet coalescence", Phys. Rev. Lett. 95, 164503 (2005).

[59] L. Duchemin, J. Eggers, and C. Josserand, "Inviscid coalescence of drops", J. Fluid Mech. 487, 167-178 (2003).

[60] S. T. Thoroddsen, K. Takehara, and T. G. Etoh, "The coalescence speed of a pendent and a sessile drop", J. Fluid Mech. 527, 85-114 (2005).

[61] S. T. Thoroddsen, B. Qian, T. G. Etoh, and K. Takehara, "The initial coalescence of miscible drops", Phys. Fluids 19, 072110 (2007).

[62] F. H. Zhang, E. Q. Li, and S. T. Thoroddsen, "Satellite formation during coalescence of unequal size drops", Phys. Rev. Lett. 102, 104502 (2009).

[63] R. B. J. Koldeweij, B. F. van Capelleveen, D. Lohse, and C. W. Visser, "Marangoni-driven spreading of miscible liquids in the binary pendant drop geometry", Soft Matter 15, 8525 (2019).

[64] S. Karpitschka and H. Riegler, "Quantitative experimental study on the transition between fast and delayed coalescence of sessile droplets with different but completely miscible liquids", Langmuir 26, 11823-11829 (2010).

[65] N. F. Morrison and O. G. Harlen, "Viscoelasticity in inkjet printing", Rheol. Acta 49, 619-632 (2010).

[66] S. D. Hoath, O. G. Harlen, and I. M. Hutching, "Jetting behavior of polymer solutions in drop-on-demand inkjet printing", J. Rheol. 56, 1109-1127 (2012).

[67] S. D. Hoath, D. C. Vadillo, O. G. Harlen, C. McIlroy, N. F. Morrison, W.-K. Hsiao, T. R. Tuladhar, S. Jung, G. D. Martin, and I. M. Hutching, "Inkjet printing of weakly elastic polymer solutions", J. NonNewton. Fluid. Mech. 205, 1-10 (2014). 


\section{Bibliography}

[68] C. W. Visser, T. Kamperman, L. P. Karbaat, D. Lohse, and M. Karperien, "In-air microfluidics enables rapid fabrication of emulsions, suspensions, and 3D modular (bio)materials", Sci. Adv. 4, eaao1175 (2018).

[69] P.-G. de Gennes, F. Brochard-Wyart, and D. Quéré, Capillarity and Wetting Phenomena: Drops, Bubbles, Pearls, Waves (Springer, New York) (2004).

[70] D. Vella and L. Mahadevan, "The "Cheerios" effect", Am. J. Phys. 73, $817-825$ (2005).

[71] S. Karpitschka, A. Pandey, L. A. Lubbers, J. H. Weijs, L. Botto, S. Das, B. Andreotti, and J. H. Snoeijer, "Liquid drops attract or repel by the inverted Cheerios effect", Proc. Natl. Acad. Sci. U.S.A. 113, 7403-7407 (2016).

[72] A. Pandey, S. Karpitschka, L. A. Lubbers, J. H. Weijs, L. Botto, S. Das, B. Andreotti, and J. H. Snoeijer, "Dynamical theory of the inverted Cheerios effect", Soft Matter 13, 6000-6010 (2017).

[73] A. Chakrabarti, L. Ryan, M. K. Chaudhury, and L. Mahadevan, "Elastic Cheerios effect: Self-assembly of cylinders on a soft solid", EPL 112, 54001 (2015).

[74] J. H. Snoeijer, "Analogies between elastic and capillary interfaces", Phys. Rev. Fluids 1, 060506 (2016).

[75] N. Bowden, A. Terfort, J. Carbeck, and G. M. Whitesides, "Selfassembly of mesoscale objects into ordered two-dimensional arrays", Science 276, 233-235 (1997).

[76] N. Bowden, I. S. Choi, B. A. Grzybowski, and G. M. Whitesides, "Mesoscale self-assembly of hexagonal plates using lateral capillary forces: synthesis using the "capillary" bond", J. Am. Chem. Soc. 121, 5373-5391 (1999).

[77] N. Bowden, S. R. J. Oliver, and G. M. Whitesides, "Mesoscale selfassembly: capillary bonds and negative menisci", J. Phys. Chem. B 104, 2714-2724 (2000).

[78] D. B. Wolfe, A. Snead, C. Mao, N. B. Bowden, and G. M. Whitesides, "Mesoscale self-assembly: capillary interactions when positive and negative menisci have similar amplitudes", Langmuir 19, 2206-2214 (2003). 
[79] M. Sokuler, G. K. Auernhammer, M. Roth, C. Liu, E. Bonacurrso, and H.-J. Bütt, "The softer the better: Fast condensation on soft surfaces", Langmuir 26, 1544-1547 (2010).

[80] J. B. Boreyko, G. Polizos, P. G. Datskos, S. A. Sarles, and C. P. Collier, "Air-stable droplet interface bilayers on oil-infused surfaces", Proc. Natl. Acad. Sci. U.S.A. 111, 7588-7593 (2014).

[81] M. K. Kim, "Principles and techniques of digital holographic microscopy", SPIE Reviews 1, 018005 (2010).

[82] A. Chakrabarti and M. K. Chaudhury, "Elastocapillary interaction of particles on the surfaces of ultrasoft gels: a novel route to study selfassembly and soft lubrication", Langmuir 30, 4684-4693 (2014).

[83] A. Pandey, C. L. Nawijn, and J. H. Snoeijer, "Hydrogel menisci: shape, interaction, and instability", Europhysics Letters 122, 36006 (2018).

[84] A. Carlson, P. Kim, G. Amberg, and H. A. Stone, "Short and long time drop dynamics on lubricated substates", EPL 104, 34008 (2013).

[85] F. Reizman, "Optical thickness measurement of thin transparent films on silicon", J. Appl. Phys 36, 3804-3807 (1965).

[86] J. D. Smith, R. Dhiman, S. Anand, E. Reza-Garduno, R. E. Cohen, G. H. McKinley, and K. K. Varanasi, "Droplet mobility on lubricantimpregnated surfaces", Soft Matter 9, 1772-1780 (2013).

[87] F. Schellenberger, J. Xie, N. Encinas, A. Hardy, M. Klapper, P. Papadopoulos, H.-J. Butt, and D. Vollmer, "Direct observation of drops on slippery lubricant-infused surfaces", Soft Matter 11, 7617-7626 (2015).

[88] T. Salez, J. D. McGraw, O. Bäumchen, K. Dalnoki-Veress, and E. Raphaël, "Capillary-driven flow induced by a stepped perturbation atop a viscous film", Phys. Fluids 24, 102111 (2012).

[89] J. D. McGraw, T. Salez, O. Bäumchen, E. Raphaël, and K. DalnokiVeress, "Self-similarity and energy dissipation in stepped polymer films", Phys. Rev. Lett. 109, 128303 (2012).

[90] O. E. Jensen, G. P. Chini, and J. R. King, "Thin-film flows near isolated humps and interior corners", J. Eng. Math. 50, 289-309 (2004). 


\section{Bibliography}

[91] R. W. Schmitt, "Double diffusion in oceanography", Annu. Rev. Fluid Mech. 26, 255-285 (1994).

[92] M. Kuang, L. Wang, and Y. Song, "Controllable printing droplets for high-resolution patterns", Adv. Mater. 26, 6950-6958 (2014).

[93] A. Fraters, M. van den Berg, Y. de Loore, H. Reinten, H. Wijshoff, D. Lohse, M. Versluis, and T. Segers, "Inkjet nozzle failure by heterogeneous nucleation: Bubble entrainment, cavitation, and diffusive growth", Phys. Rev. Appl. 12, 064019 (2019).

[94] T. Gambaryan-Roisman, "Liquids on porous layers: wetting, imbibition and transport processes", Curr. Opin. Colloid Interface Sci. 19, 320-335 (2014).

[95] I. Szilagyi, A. Sadeghpour, and M. Borkovec, "Destabilization of colloidal suspensions by multivalent ions and polyelectrolytes: from screening to overcharging", Langmuir 28, 6211-6215 (2012).

[96] E. J. W. Verwey and J. T. G. Overbeek, Theory of the stability of lyophobic colloids (Elsevier Publishing Company, Amsterdam) (1948).

[97] H. Hamada and D. W. Bousfield, "Effect of cationic additives on ink penetration", JPPS 35, 118-122 (2009).

[98] T. Liu and R. J. Nick, "Modified pigment containing inkjet ink compositions having a reduced conductivity increase", (U.S. Patent 8,147,604 B2, 2012).

[99] D. Okamura, K. Yamasaki, K. Moribe, K. Okumura, K. Shiiba, and H. Kishi, "Ink jet ink, ink cartridge, and ink jet recording method", (U.S. Patent 9,243,155 B2, 2016).

[100] F. K. Hansen and G. Rødsrud, "Surface tension by pendant drop", J. Colloid Interface Sci 140, 1-8 (1991).

[101] C.-Y. Tan and Y.-X. Huang, "Dependence of refractive index on concentration and temperature in electrolyte solution, polar solution, nonpolar solution, and protein solution", J. Chem. Eng. Data 60, 2827-2833 (2015). 
[102] H.-L. Zhang, G.-H. Chen, and S.-J. Han, "Viscosity and density of $\mathrm{H}_{2} \mathrm{O}+\mathrm{NaCl}+\mathrm{CaCl}_{2}$ and $\mathrm{H}_{2} \mathrm{O}+\mathrm{KCl}+\mathrm{CaCl}_{2}$ at $298.15 \mathrm{~K}$ ", J. Chem. Eng. Data 42, 526-530 (1997).

[103] K. G. Winkels, J. H. Weijs, A. Eddi, and J. H. Snoeijer, "Initial spreading of low-viscosity drops on partially wetting surfaces", Phys. Rev. E 85, 055301 (2012).

[104] B. B. J. Stapelbroek, H. P. Jansen, E. S. Kooij, J. H. Snoeijer, and A. Eddi, "Universal spreading of water drops on complex surfaces", Soft Matter 10, 2641-2648 (2014).

[105] J. F. Hernández-Sánchez, A. Eddi, and J. H. Snoeijer, "Marangoni spreading due to a localized alcohol supply on a thin water film", Phys. Fluids 27, 032003 (2015).

[106] X. Wang, E. Bonaccurso, J. Venzmer, and S. Garoff, "Deposition of drops containing surfactants on liquid pools: Movement of the contact line, Marangoni ridge, capillary waves and interfacial particles", Colloids Surf. 486, 53-59 (2015).

[107] I. B. Liu, N. Sharifi-Mood, and K. J. Stebe, "Curvature-driven assembly in soft matter", Phil. Trans. R. Soc. A 374, 20150133 (2016).

[108] L. H. N. Nukui, L. R. S. Barbosa, and D. F. S. Petri, "Impact of monovalent and divalent cations on the colloidal stability of negatively charged latex particles decorated with poly(ethyleneglycol)", Ind. Eng. Chem. Res. 66, 606-614 (2016).

[109] P. G. de Gennes, "Solvent evaporation of spin cast films: "crust" effects", Eur. Phys. J. E 7, 31-34 (2002).

[110] F. Box, D. O'Kiely, O. Kodio, M. Inizan, A. A. Castrejón-Pita, and D. Vella, "Dynamics of wrinkling in ultrathin elastic sheets", Proc. Natl. Acad. Sci. U.S.A. 116, 20875-20880 (2019).

[111] V. M. Starov and H.-J. Butt, "Editorial overview: Worldwide increasing interest in wetting phenomena", Curr. Opin. Colloid Interface Sci. 36, A1-A4 (2018).

[112] M. E. J. Haagh, N. Schilderink, F. Mugele, and M. H. G. Duits, "Wetting of mineral surfaces by fatty-acid-laden oil and brine: carbonate effect at elevated temperature", Energy Fuels 33, 9446-9456 (2019). 


\section{Bibliography}

[113] D. Bonn, J. Eggers, J. Indekeu, J. Meunier, and E. Rolley, "Wetting and spreading", Rev. Mod. Phys. 81, 739-805 (2009).

[114] J. W. Drelich, L. Boinovich, E. Chibowski, C. D. Volpe, L. Hołysz, A. Marmur, and S. Siboni, "Contact angles: history of over 200 years of open questions", Surface Innovations 8, 3-27 (2020).

[115] A. F. M. Leenaars, J. A. M. Huethorst, and J. J. van Oekel, "Marangoni drying: a new extremely clean drying process", Langmuir 6, 1701-1703 (1990).

[116] O. K. Matar and R. V. Craster, "Dynamics of surfactant-assisted spreading", Soft Matter 5, 3801-3809 (2009).

[117] H. Tan, C. Diddens, P. Lv, J. G. M. Kuerten, X. Zhang, and D. Lohse, "Evaporation-triggered microdroplet nucleation and the four life phases of an evaporating Ouzo drop", Proc. Natl. Acad. Sci. U.S.A 113, 8642 8647 (2016).

[118] K. Sefiane, S. David, and M. E. R. Shanahan, "Wetting and evaporation of binary mixture drops", J. Phys. Chem. B 112, 11317-11323 (2008).

[119] A. M. J. Edwards, P. S. Atkinson, C. S. Cheung, H. Liang, D. J. Fairhurst, and F. F. Ouali, "Density-driven flows in evaporating binary liquid droplets", Phys. Rev. Lett. 121, 184501 (2018).

[120] Y. Li, C. Diddens, P. Lv, H. Wijshoff, M. Versluis, and D. Lohse, "Gravitational effect in evaporating binary microdroplets", Phys. Rev. Lett. 122, 114501 (2019).

[121] S. M. Hajji, M. B. Errahmani, R. Coudert, R. R. Durand, A. Cao, and E. Taillandier, "A comparative study of hexanediol-1,2 and octanetriol-1,2,3 in aqueous solutions by different physical techniques", J. Phys. Chem. 93, 4819-4824 (1989).

[122] M. Frindi, B. Michels, and R. Zana, "Ultrasonic absorption studies of surfactant exchange between micelles and bulk phase in aqueous micellar solutions of nonionic surfactants with short alkyl chains 1.1,2-hexanediol and 1,2,3-octanetriol", J. Phys. Chem. 95, 4832-4837 (1991).

[123] N. Székely, L. Almásy, A. Rădulescu, and L. Rosta, "Small-angle neutron scattering study of aqueous solutions of pentanediol and hexanediol", J. Appl. Cryst. 40, s307-s311 (2007). 
[124] Y. H. Tan and J. A. Finch, "Frothers and gas dispersion: a review of the structure-property function relationship", Physicochem. Probl. Miner. Process. 54, 40-53 (2018).

[125] C. M. Romero, M. S. Páez, J. A. Miranda, D. J. Hernández, and L. E. Oviedo, "Effect of temperature on the surface tension of diluted aqueous solutions of 1,2-hexanediol, 1,5-hexanediol, 1,6-hexanediol and 2,5-hexanediol", Fluid Ph. Equilibria 258, 67-72 (2007).

[126] N. J. Cira, A. Benusiglio, and M. Prakash, "Vapour-mediated sensing and motility in two-component droplets", Nature 519, 446-450 (2015).

[127] A. Benusiglio, N. J. Cira, and M. Prakash, "Two-component Marangonicontracted droplets: friction and shape", Soft Matter 14, 7724-7730 (2018).

[128] H. Fujiwara, Spectroscopic Ellipsometry: Principles and Applications (John Wiley \& Sons) (2007).

[129] V. J. Novotny and A. Marmur, "Wetting autophobicity", J. Colloid Interface Sci. 145, 355-361 (1991).

[130] J. Marra and J. A. M. Huethorst, "Physical principles of Marangoni drying", Langmuir 7, 2748-2755 (1991).

[131] A. P. Mouat, C. E. Wood, J. E. Pye, and J. C. Burton, "Tuning contact line dynamics and deposition patterns in volatile liquid mixtures", Phys. Rev. Lett. 124, 064502 (2020).

[132] S. Semenov, A. Trybala, R. G. Rubio, N. Kovalchuk, V. Starov, and M. G. Velarde, "Simultaneous spreading and evaporation: recent developments", Adv. Colloid Interface Sci. 206, 382-398 (2014).

[133] P. J. Scales, F. Grieser, D. N. Furlong, and T. W. Healy, "Contact angle changes for hydrophobic and hydrophilic surfaces induced by nonionic surfactants", Colloids Surf. 21, 55-68 (1986).

[134] B.-Y. Zhu and T. Gu, "Surfactant adsorption at solid-liquid interfaces", Adv. Colloid Interface Sci 37, 1-32 (1991).

[135] H. Rupprecht and T. Gu, "Structure of adsorption layers of ionic surfactants at the solid/liquid interface", Colloid Polym. Sci. 269, 506-522 (1991). 


\section{Bibliography}

[136] W. R. Birch, M. A. Knewtson, S. Garoff, R. M. Suter, and S. Satija, "The molecular stucture of autophobed monolayers and precursing films of a cationic surfactant on the silicon oxide/silicon surface", Colloids Surf. A 89, 145-155 (1994).

[137] W. R. Birch, M. A. Knewtson, S. Garoff, R. M. Suter, and S. Satija, "Structure of precursing thin films of an anionic surfactant on a silicon oxide/silicon surface", Langmuir 11, 48-56 (1995).

[138] U. Thiele, J. H. Snoeijer, S. Trinschek, and K. John, "Equilibrium contact angle and adsorption layer properties with surfactants", Langmuir 34, 7210-7221 (2018).

[139] A. B. Afsar-Siddiqui, P. F. Luckham, and O. K. Matar, "Dewetting behavior of aqueous cationic surfactant solutions on liquid films", Langmuir 20, 7575-7582 (2004).

[140] U. Thiele, D. V. Todorova, and H. Lopez, "Gradient dynamics description for films of mixtures and suspensions: Dewetting triggered by coupled film height and concentration fluctuations", Phys. Rev. Lett. 111, 117801 (2013).

[141] B. Bera, M. H. G. Duits, M. A. C. Stuart, D. van den Ende, and F. Mugele, "Surfactant induced autophobing", Soft Matter 12, 4562 4571 (2016).

[142] B. Bera, O. Carrier, E. H. G. Backus, M. Bonn, N. Shahidzadeh, and D. Bonn, "Counteracting interfacial energetics for wetting of hydrophobic surfaces in the presence of surfactants", Langmuir 34, 1234412349 (2018).

[143] A. Marmur and M. D. Lelah, "The spreading of aqueous surfactant solutions on glass", Chem. Eng. Commun. 13, 133-143 (1981).

[144] B. Frank and S. Garoff, "Surfactant self-assembly near contact lines: control of advancing surfactant solutions", Colloids Surf. A 116, 31-42 (1996).

[145] Y. Takenaka, Y. Sumino, and T. Ohzono, "Dewetting of a droplet induced by the adsorption of surfactants on a glass substrate", Soft Matter 10, 5597-5602 (2014). 
[146] X. Zhong and F. Duan, "Dewetting transition induced by surfactants in sessile droplets at the early evaporation stage", Soft Matter 12, 508-513 (2016).

[147] R. Tadmor, A. Baksi, S. Gulec, S. Jadhav, H. E. N'guessan, K. Sen, V. Somasi, M. Tadmor, P. Wasnik, and S. Yadav, "Drops that change their mind: spontaneous reversal from spreading to retraction", Langmuir 35, 15734-15738 (2019).

[148] J. G. Kirkwood and J. Riseman, "The intrinsic viscosities and diffusion constants of flexible macromolecules in solution", J. Chem. Phys. 16, 565-573 (1948).

[149] E. L. Decker, B. Frank, Y. Suo, and S. Garoff, "Physics of contact angle measurements", Colloids Surf. A 156, 177-189 (1999).

[150] B. Frank and S. Garoff, "Temporal and spatial development of surfactant self-assemblies controlling spreading of surfactant solutions", Langmuir 11, 4333-4340 (1995).

[151] P. Jarosiewicz, G. Czechowski, and J. Jadżyn, "The viscous properties of diols V. 1,2-hexanediol in water and butanol solutions", Z. Naturforsch. A 59a, 559-562 (2004).

[152] B. Smit, A. G. Schlijper, L. A. M. Rupert, and N. M. van Os, "Effects of chain length of surfactants on the interfacial tension: molecular dynamics simulations and experiments", J. Phys. Chem. 94, 6933-6935 (1990).

[153] J. Hu, X.-D. Xiao, D. F. Ogletree, and M. Salmeron, "Imaging the condensation and evaporation of molecularly thin films of water with nanometer resolution", Science 268, 267-269 (1995).

[154] J. A. M. Huethorst and J. Marra, "Motion of Marangoni-contracted water drops across inclined hydrophilic surfaces", Langmuir 7, 27562763 (1991).

[155] W. W. Grabowski and L.-P. Wang, "Growth of cloud droplets in a turbulent environment", Annu. Rev. Fluid Mech. 45, 293-324 (2013).

[156] K. M. Wisdom, J. A. Watson, X. Qu, F. Liu, G. S. Watson, and C.H. Chen, "Self-cleaning of superhydrophobic surfaces by self-propelled 


\section{Bibliography}

jumping condensate", Proc. Natl. Acad. Sci. U.S.A. 20, 7992-7997 (2013).

[157] G. S. Watson, M. Gellender, and J. A. Watson, "Self-propulsion of dew drops on lotus leaves: a potential mechanism for self-cleaning", Biofouling 30, 427-434 (2014).

[158] R. D. Narhe, D. A. Beysens, and Y. Pomeau, "Dynamics drying in the early-stage coalescence of droplets sitting on a plate", Eur. Phys. Lett. 81, 46002 (2008).

[159] U. Delabre and A.-M. Cazabat, "Coalescence driven by line tension in thin nematic films", Phys. Rev. Lett. 104, 227801 (2010).

[160] S. Anand, A. T. Paxson, R. Dhiman, J. D. Smith, and K. K. Varanasi, "Enhanced condensation on lubricant-impregnated nanotextured surfaces", ACS Nano 6, 10122-10129 (2012).

[161] P. Kim, T.-S. Wong, J. Alvarenga, M. J. Kreder, W. E. AdornoMartinez, and J. Aizenberg, "Liquid-infused nanostructured surfaces with extreme anti-ice and anti-frost performance", ACS Nano 6, 65696577 (2012).

[162] M. A. Hack, M. Costalonga, T. Segers, S. Karpitschka, H. Wijshoff, and J. H. Snoeijer, "Printing wet-on-wet: Attraction and repulsion of drops on a viscous film", Appl. Phys. Lett. 113, 183701 (2018).

[163] D. T. Wasan, S. M. Shah, N. Aderangi, M. S. Chan, and J. J. McNamara, "Observations on the coalescence behavior of oil droplets and emulsion stability in enhanced oil recovery", SPE J. 18, 409-417 (1978).

[164] D. T. Wasan, J. J. McNamara, S. M. Shah, K. Sampath, and N. Aderangi, "The role of coalescence phenomena and interfacial rheological properties in enhanced oil recovery: An overview", J. Rheol. 23, 181-207 (1979).

[165] J. M. Shaw, "A microscopic view of oil slick break-up and emulsion formation in breaking waves", Spill Sci. Technol. Bull. 8, 491-501 (2003).

[166] J. Kamp, J. Villwock, and M. Kraume, "Drop coalescence in technical liquid/liquid applications: a review on experimental techniques and modeling approaches", Rev. Chem. Eng. 33, 1-47 (2016). 
[167] I. Langmuir, "Oil lenses on water and the nature of monomolecular expanded films", J. Chem. Phys. 1, 756-776 (1933).

[168] R. F. Day, E. J. Hinch, and J. R. Lister, "Self-similar capillary pinchoff of an inviscid fluid", Phys. Rev. Lett. 80, 704-707 (1998).

[169] T. Erneux and S. H. Davis, "Nonlinear rupture of free films", Phys. Fluids A 5, 1117-1122 (1993).

[170] B. Scheid, E. A. van Nierop, and H. A. Stone, "Thermocapillary-assisted pulling of contact-free liquid films", Phys. Fluids 24, 032107 (2012).

[171] J. Eggers and M. A. Fontelos, Singularities: Formation, Structure, and Propagation (Cambridge University Press) (2015).

[172] M. Heil and A. L. Hazel, "OOMPH-LIB-an object-oriented multi-physics finite-element library", in Fluid-structure interaction, 19-49 (Springer) (2006).

[173] L. Ting and J. B. Keller, "Slender jets and thin sheets with surface tension", SIAM J. Appl. Math. 50, 1533-1546 (1990).

[174] J. Eggers, "Universal pinching of 3d axisymmetric free-surface flow", Phys. Rev. Lett. 71, 3458-3460 (1993).

[175] N. Laan, K. G. de Bruin, D. Bartolo, C. Josserand, and D. Bonn, "Maximum diameter of impacting liquid droplets", Phys. Rev. Appl. 2, 044018 (2014).

[176] M. A. Hack, W. Tewes, Q. Xie, C. Datt, K. Harth, J. Harting, and J. H. Snoeijer, "Self-similar liquid lens coalescence", Phys. Rev. Lett. 124, $194502(2020)$.

[177] R. W. Hopper, "Plane Stokes flow driven by capillarity on a free surface", J. Fluid Mech. 213, 349-375 (1990).

[178] R. I. Tanner, Engineering rheology, volume 52 (OUP Oxford) (2000).

[179] M. Renardy, Mathematical analysis of viscoelastic flows (SIAM) (2000).

[180] E. Hinch, "The flow of an Oldroyd fluid around a sharp corner", J. NonNewton. Fluid Mech. 50, 161-171 (1993). 


\section{Bibliography}

[181] M. Renardy, "The stresses of an upper convected Maxwell fluid in a Newtonian velocity field near a re-entrant corner", J. NonNewton. Fluid Mech. 50, 127-134 (1993).

[182] J. Evans and D. Sibley, "Re-entrant corner flows of PTT fluids in the Cartesian stress basis", J. Non-Newton. Fluid Mech. 153, 12-24 (2008).

[183] G. Astarita and G. Apuzzo, "Motion of gas bubbles in non-Newtonian liquids", AIChE Journal 11, 815-820 (1965).

[184] Y. J. Liu, T. Y. Liao, and D. D. Joseph, "A two-dimensional cusp at the trailing edge of an air bubble rising in a viscoelastic liquid", J. Fluid Mech. 304, 321-342 (1995).

[185] R. Zenit and J. Feng, "Hydrodynamic interactions among bubbles, drops, and particles in non-Newtonian liquids", Annu. Rev. Fluid Mech. 50, 505-534 (2018).

[186] A. V. Bazilevskii, V. M. Entov, and A. N. Rozhkov, "Liquid filament microrheometer and some of its applications", in Proceedings of the Third European Rheology Conference, edited by D. R. Oliver, 41 (Elsevier Applied Science) (1990).

[187] V. M. Entov and E. J. Hinch, "Effect of a spectrum of relaxation times on the capillary thinning of a filament of elastic liquid", J. NonNewton. Fluid Mech. 72, 31-53 (1997).

[188] S. L. Anna and G. H. McKinley, "Elasto-capillary thinning and breakup of model elastic liquids", J. Rheol. 45, 115-138 (2001).

[189] Y. Amarouchene, D. Bonn, J. Meunier, and H. Kellay, "Inhibition of finite-time singularity during droplet fission of a polymeric fluid", Phys. Rev. Lett. 86, 3558-3561 (2001).

[190] J. Eggers, M. A. Herrada, and J. H. Snoeijer, "Self-similar breakup of polymeric threads as described by the Oldroyd-B model", J. Fluid Mech. 887, A19 (2020).

[191] A. Deblais, M. A. Herrada, J. Eggers, and D. Bonn, "Self-similarity in the breakup of very dilute viscoelastic solutions", J. Fluid Mech. 904, R2 (2020). 
[192] M. Abkarian and H. A. Stone, "Stretching and break-up of saliva filaments during speech: A route for pathogen aerosolization and its potential mitigation", Phys. Rev. Fluids 5, 102301 (2020).

[193] M. Renardy, "Self-similar breakup of non-Newtonian liquid jets", Rheology Reviews 2, 171-196 (2004).

[194] S. C. Varma, A. Saha., S. Mukherjee, A. Bandopadhyay, A. Kumar, and S. Chakraborty, "Universality in coalescence of polymeric fluids", Soft Matter 16, 10921-10927 (2020).

[195] P. Yue, J. J. Feng, C. Liu, and J. Shen, "Diffuse-interface simulations of drop coalescence and retraction in viscoelastic fluids", J. NonNewton. Fluid Mech. 129, 163-176 (2005).

[196] A. Zdravkov, G. Peters, and H. Meijer, "Film drainage between two captive drops: PEO-water in silicon oil", J. Colloid Interface Sci. 266, 195-201 (2003).

[197] A. Menchaca-Rocha, A. Martínez-Dávalos, R. N. nez, S. Popinet, and S. Zaleski, "Coalescence of liquid drops by surface tension", Phys. Rev. E 63, 046309 (2001).

[198] M. Wu, T. Cubaud, and C.-M. Ho, "Scaling law in liquid drop coalescence driven by surface tension", Phys. Fluids 16, L51-L54 (2004).

[199] W. Yao, H. J. Maris, P. Pennington, and G. M. Seidel, "Coalescence of viscous liquid drops", Phys. Rev. E 71, 016309 (2005).

[200] R. B. Bird, C. F. Curtiss, R. C. Armstrong, and O. Hassager, Dynamics of polymeric liquids (John Wiley \& Sons) (1987).

[201] V. Gauri and K. W. Koelling, "Extensional rheology of concentrated poly(ethylene oxide) solutions", Rheol. Acta 36, 555-567 (1997).

[202] C. E. Stauffer, "The measurement of surface tension by the pendant drop technique", J. Phys. Chem. 69, 1933-1938 (1965).

[203] J. Qian and C. K. Law, "Regimes of coalescence and separation in droplet collision", J. Fluid Mech. 331, 59-80 (1997).

[204] C. Wang, S. Fu, L. Kung, and C. Law, "Combustion and microexplosion of collision-merged methanol/alkane droplets", Proc. Combust. Inst. 30, 1965-1972 (2005). 


\section{Bibliography}

[205] R.-H. Chen, "Diesel-diesel and diesel-ethanol drop collisions", Appl. Therm. Eng. 27, 604-610 (2007).

[206] J. Jiang, G. Shea, P. Rastogi, T. Kamperman, C. H. Venner, and C. W. Visser, "Continuous high-throughput fabrication of architected micromaterials via in-air photopolymerization", Adv. Mater. 33, 2006336 (2021).

[207] I. V. Roisman, "Dynamics of inertia dominated binary drop collisions", Phys. Fluids 16, 3438-3449 (2004).

[208] C. Tang, P. Zhang, and C. K. Law, "Bouncing, coalescence, and separation in head-on collision of unequal-size droplets", Phys. Fluids 24, 022101 (2012).

[209] F. Blanchette, "Simulation of mixing within drops due to surface tension variations", Phys. Rev. Lett. 105, 074501 (2010).

[210] C. Planchette, E. Lorenceau, and G. Brenn, "Liquid encapsulation by binary collisions of immiscible liquid drops", Colloids Surf. 365, 89-94 (2010).

[211] T.-C. Gao, R.-H. Chen, J.-Y. Pu, and T.-H. Lin, "Collision between an ethanol drop and a water drop", Exp. Fluids 38, 731-738 (2005).

[212] J. Kohno, M. Kobayashi, and T. Suzuki, "Protrusion formation during the collisional process of ethanol and water droplets: Capillary wave propagation on the water droplet", Chem. Phys. Lett. 578, 15-20 (2013).

[213] T. Suzuki and J. Kohno, "Simultaneous detection of images and Raman spectra of colliding droplets: composition analysis of protrusions emerging during collisions of ethanol and water droplets", J. Phys. Chem. B 118, 5781-5786 (2014).

[214] G. Vazquez, E. Alvarez, and J. M. Navaza, "Surface tension of alcohol + water from 20 to $50{ }^{\circ} \mathrm{C} "$, J. Chem. Eng. Data 40, 611-614 (1995).

[215] B. Gónzalez, N. Calvar, E. Gómez, and A. Domínguez, "Density, dynamic viscosity, and derived properties of binary mixtures of methanol or ethanol with water, ethyl acetate, and methyl acetate at $\mathrm{T}=$ (293.15, 298.15, and 303.15) K", J. Chem. Thermodynamics 39, 1578 1588 (2007). 
[216] S. T. Thoroddsen and K. Takehara, "The coalescence cascade of a drop", Phys. Fluids 12, 1265-1267 (2000).

[217] S. T. Thoroddsen, T. G. Etoh, and K. Takehara, "Air entrapment under an impacting drop", J. Fluid Mech. 478, 125-134 (2003).

[218] T. Tran, H. de Maleprade, C. Sun, and D. Lohse, "Air entrainment during impact of droplets on liquid surfaces", J. Fluid Mech. 726, R3 (2013).

[219] M. H. W. Hendrix, W. Bouwhuis, D. van der Meer, D. Lohse, and J. H. Snoeijer, "Universal mechanism for air entrainment during liquid impact", J. Fluid Mech. 789, 708-725 (2016).

[220] R. A. Cairncross, P. R. Schunk, T. A. Baer, R. R. Rao, and P. A. Sackinger, "A finite element method for free surface flows of incompressible fluids in three dimensions. part I. boundary fitted mesh motion", Int. J. Numer. Methods Fluids 33, 375-403 (2000).

[221] Y. Li, C. Diddens, A. Prosperetti, K. L. Chong, X. Zhang, and D. Lohse, "Bouncing oil droplet in a stratified liquid and its sudden death", Phys. Rev. Lett. 122, 154502 (2019).

[222] S. Par̆ez, G. Guevara-Carrion, H. Hasse, and J. Vrabec, "Mutual diffusion in the ternary mixture of water + methanol + ethanol and its binary subsystems", Phys. Chem. Chem. Phys. 15, 3985-4001 (2013).

[223] N. Ashgriz and J. Y. Poo, "Coalescence and separation in binary collisions of liquid drops", J. Fluid Mech. 221, 183-204 (1990).

[224] X. Chen, S. Mandre, and J. Feng, "Partial coalescence between a drop and a liquid-liquid interface", Phys. Fluids 18, 051705 (2006).

[225] K. Sun, P. Zhang, Z. Che, and T. Wang, "Marangoni-flow-induced partial coalescence of a droplet on a liquid-air interface", Phys. Rev. Fluids 3, 023602 (2018).

[226] H. Ding, E. Q. Li, F. H. Zhang, Y. Sui, P. D. M. Spelt, and S. T. Thoroddsen, "Propagation of capillary waves and ejection of small droplets in rapid droplet spreading", J. Fluid Mech. 697, 92-114 (2012). 


\section{Bibliography}

[227] T. Gilet, K. Mulleners, J. P. Lecomte, N. Vandewalle, and S. Dorbolo, "Critical parameters for the partial coalescence of a droplet", Phys. Rev. E 75, 036303 (2007).

[228] C. R. Constante-Amores, A. Batchvarov, L. Kahouadji, S. Shin, J. Chergui, D. Juric, and O. K. Matar, arXiv (2021), https://arxiv.org/abs/2102.08071.

[229] C. Huh, M. Inoue, and S. G. Mason, "Uni-directional spreading of one liquid on the surface of another", Can. J. Chem. Eng. 53, 367-371 (1975).

[230] D. P. Hoult, "Oil spreading on the sea", Annu. Rev. Fluid Mech. 4, 341-368 (1972).

[231] M. Foda and R. G. Cox, "The spreading of thin liquid films on a waterair interface", J. Fluid Mech. 101, 33-51 (1980).

[232] S. Berg, "Marangoni-driven spreading along liquid-liquid interfaces", Phys. Fluids 21, 032105 (2009).

[233] R. D. Davis, M. I. Jacobs, F. A. Houle, and K. R. Wilson, "Collidingdroplet microreactor: rapid on-demand inertial mixing and metalcatalyzed aqueous phase oxidation processes", Anal. Chem 89, 1249412501 (2017).

[234] A. Frohn and N. Roth, Dynamics of Droplets (Springer, New York) (2000).

[235] D. Daniel, J. V. I. Timonen, R. Li, S. J. Velling, and J. Aizenberg, "Oleoplaning droplets on lubricated surfaces", Nat. Phys. 13, 1020-1026 (2017).

[236] A. W. Wray, O. Matar, and D. T. Papageorgiou, "Non-linear waves in electrified viscous film flow down a vertical cylinder", IMA J. Appl. Math. 77, 430-440 (2012).

[237] R. T. van Gaalen, C. Diddens, H. M. A. Wijshoff, and J. G. M. Kuerten, "The evaporation of surfactant-laden droplets: A comparison between contact line models", J. Colloid Interface Sci. 579, 888-897 (2020). 



\section{Summary}

This thesis explored a variety of wetting and coalescence flows in multi-phase systems. Such flows are ubiquitous in nature and technology alike. In particular, the research presented in this thesis was inspired by several problems encountered in inkjet printing. A good understanding of wetting and coalescence is crucial for the successful application of inkjet printing technology and for its future development towards, e.g., additive manufacturing.

In part I of this thesis, we studied various wetting phenomena, which we broadly defined as the behaviour of (multiple) drops on a variety of substrates.

In chapter 2 , we studied the capillary interaction between two drops placed close together on a thin viscous film. We showed that this interaction arises due to visco-capillary waves that are induced by the presence of the drops. The interaction is non-monotonic: both attractive and repulsive interactions were observed depending on the distance separating the two drops. Using the thin-film equation, we identified the scaling law for the spreading of the visco-capillary waves and demonstrated that this governs the range over which the interaction is observed. This confirms the direct connection between the shape of the visco-capillary waves and the interaction type.

In chapter 3 , we continued our study of drops on thin liquid films. We revealed that a colloidal drop forms a ring-shaped pattern when deposited on a thin saline water film. The ring consists of particle clusters - the electrostatically stabilised colloidal particles in the drop destabilise when brought into contact with cations in the saline water film. The shape of the pattern is largely determined by an undulation of the contact line that appears upon first contact of the drop with the liquid film. Finally, we revealed that the clusters that constitute the ring are transported radially outwards by Marangoni flow.

In chapter 4, we studied the wetting properties of two-component drops that consist of mixtures of vicinal alkanediols and water. These diols behave surfactant-like in water. However, the contact angles of such mixtures on solid substrates are surprisingly large. Using experiments, we revealed that 
the contact angle is determined by two separate mechanisms of completely different nature, namely Marangoni contraction (hydrodynamic) and autophobing (molecular). The competition between these effects can even inhibit Marangoni contraction, highlighting the importance of molecular structures in physico-chemical hydrodynamics.

In part II of this thesis, we studied various coalescence problems. We first studied the coalescence of drops floating on a liquid substrate, and subsequently turned our attention to the coalescence of multi-component drops.

In chapter 5, we experimentally and theoretically revealed the dynamics of drop coalescence on a thick layer of a low viscosity liquid. We showed that these so-called "liquid lenses" merge by the self-similar vertical growth of a bridge connecting the two lenses. Using a slender analysis, we derived similarity solutions corresponding to the viscous and inertial limits. Excellent agreement was found with the experiments without any adjustable parameters, capturing both the spatial and temporal structures of the flow during coalescence. Finally, we considered the crossover between the two regimes, and showed that all data of different lens viscosities collapse on a single curve capturing the full range of the coalescence dynamics.

In chapter 6 , we continued our study of coalescing liquid lenses, focusing on coalescence in the viscous regime. We showed that the bridge dynamics follow a self-similar solution at leading order, but, depending on the largescale boundary conditions on the drop, significant corrections may arise to this solution. These dynamics were studied in detail using numerical simulations and through matched asymptotics. We revealed that liquid lens coalescence can involve a global translation of the drops, a feature that was confirmed experimentally.

In chapter 7 , we studied the coalescence of viscoelastic drops. The breakup of a drop changes dramatically when polymers are added to the liquid. With the strong elongation of the polymers during the process, long threads connecting the two drops appear prior to their eventual pinch-off. In this chapter, we demonstrated how elasticity affects drop coalescence, the complement of the much studied drop pinch-off. We revealed the emergence of an elastic singularity, characterised by a diverging interface curvature at the point of coalescence. Intriguingly, while the polymers dictate the spatial features of coalescence, we found that they hardly affect the temporal evolution of the bridge. These results were explained using a novel viscoelastic similarity analysis.

In chapter 8 , we studied the collisions between two drops of different 
surface tensions. Using experimental and numerical techniques, we revealed that the asymmetric shape evolution that is observed during such collisions is caused by asymmetric capillary waves, which are the result of the different surface tensions of the drops. We showed that the asymmetry is enhanced by increasing the surface tension difference, and suppressed by increasing the inertia of the colliding drops. Furthermore, we studied the capillary waves on coalescing drops in the limit of no inertia. We revealed that the asymmetry is not directly caused by Marangoni forces. In fact, we found that asymmetry is strongly reduced by the Marangoni effect. Rather, the different intrinsic capillary wave amplitudes and velocities associated with the different surface tensions of the drops lie at the origin of the asymmetry that is observed during drop collision and coalescence.

Finally, in chapter 9, we presented our conclusions and suggested several areas that might be of interest for future research. The results presented in this thesis could serve as inspiration for the further improvement of inkjet printing technology and its development towards applications beyond document printing. 



\section{Samenvatting}

Dit proefschrift onderzocht een verscheidenheid aan bevochtigings- en samenvloeiingsstromen in meerfasensystemen. Dergelijke stromen zijn alomtegenwoordig in zowel natuur als technologie. Het onderzoek dat in dit proefschrift wordt gepresenteerd, is in het bijzonder geïnspireerd door verschillende problemen die zich voordoen bij inkjetprinten. Een goed begrip van bevochtiging en samenvloeiing is cruciaal voor de succesvolle toepassing van inkjetprinttechnologie en voor de toekomstige ontwikkeling ervan naar bijvoorbeeld driedimensionaal printen.

In deel I van dit proefschrift hebben we verschillende bevochtigingsverschijnselen bestudeerd, die we in grote lijnen hebben gedefinieerd als het gedrag van (meerdere) druppels op een verscheidenheid aan substraten.

In hoofdstuk 2 hebben we de capillaire interactie tussen twee druppels die dicht bij elkaar op een dunne viskeuze vloeistoflaag zijn geplaatst bestudeerd. We toonden aan dat deze interactie ontstaat door viskeuze-capillaire golven die worden geïnduceerd door de aanwezigheid van de druppels. De interactie is niet-monotoon: zowel aantrekkende als afstotende interacties werden waargenomen, afhankelijk van de afstand tussen de twee druppels. Met behulp van de dunne laag-vergelijking hebben we de schalingswet voor de groei van de viskeuze-capillaire golven geïdentificeerd en aangetoond dat dit het bereik bepaalt waarover de interactie wordt waargenomen. Dit bevestigt het directe verband tussen de vorm van de viskeuze-capillaire golven en het interactietype.

In hoofdstuk 3 vervolgden we onze studie van druppels op dunne vloeistoflagen. We hebben laten zien dat een colloïdale druppel een ringvormig patroon vormt wanneer het wordt neergelegd op een dunne zoutwaterlaag. De ring bestaat uit clusters van colloïdale deeltjes - de elektrostatisch gestabiliseerde colloïdale deeltjes in de druppel destabiliseren wanneer ze in contact worden gebracht met kationen in de zoute waterlaag. De vorm van het patroon wordt grotendeels bepaald door een golving van de contactlijn die verschijnt bij het eerste contact van de druppel met de vloeistoflaag. Ten slotte hebben 
we laten zien dat de clusters die de ring vormen, radiaal naar buiten worden getransporteerd door Marangoni-stroming.

In hoofdstuk 4 hebben we de bevochtigingseigenschappen van tweecomponentendruppels die bestaan uit mengsels van vicinale alkaandiolen en water bestudeerd. Deze diolen gedragen zich als oppervlakteactieve stoffen in water. De contacthoeken van dergelijke mengsels op vaste substraten zijn echter verrassend groot. Middels experimenten hebben we onthuld dat de contacthoek wordt bepaald door twee afzonderlijke mechanismen van totaal verschillende aard, namelijk Marangoni-contractie (hydrodynamisch) en autofobische contacthoektoename (moleculair). De concurrentie tussen deze effecten kan zelfs de contractie van Marangoni-contractie onderdrukken, wat het belang van moleculaire structuren in de fysisch-chemische hydrodynamica benadrukt.

In deel II van dit proefschrift hebben we verschillende samenvloeiingsproblemen bestudeerd. We bestudeerden eerst de samenvloeiing van druppels die op een vloeibaar substraat drijven, en richtten vervolgens onze aandacht op de samenvloeiing van druppels met meerdere componenten.

In hoofdstuk 5 hebben we experimenteel en theoretisch de dynamische eigenschappen van druppelsamenvloeiing op een dikke laag vloeistof met een lage viscositeit onthuld. We toonden aan dat deze zogenaamde "vloeibare lenzen" samenvloeien door de verticale groei van een zelfgelijkvormige brug die de twee lenzen verbindt. Op basis van een dunne laag-benadering hebben we oplossingen afgeleid die overeenkomen met de viskeuze limiet en de traagheidslimiet. Er werd uitstekende overeenstemming gevonden met de experimenten zonder enige vrije parameters, waarbij zowel de ruimtelijke als temporele structuren van de stroming tijdens samenvloeiing werden vastgelegd. Ten slotte hebben we de overgang tussen de twee limieten bestudeerd en hebben we aangetoond dat alle gegevens van verschillende lensviscositeiten samenvallen op een enkele curve die alle dynamische eigenschappen van de samenvloeiing beschrijft.

In hoofdstuk 6 vervolgden we ons onderzoek naar de samenvloeiing van vloeibare lenzen, waarbij we ons concentreerden op samenvloeiing in de viskeuze limiet. We toonden aan dat de dynamische groei van de brug een zelfgelijkvormige oplossing volgt in de leidende orde, maar dat, afhankelijk van de grootschalige randvoorwaarden, significante correcties van toepassing kunnen zijn op deze oplossing. De dynamische groei werd vervolgens in detail bestudeerd met behulp van numerieke simulaties en asymptotiek. We hebben laten zien dat een globale beweging van de druppels deel kan uitmaken van de samenvloeiing van vloeibare lenzen, een kenmerk dat experimenteel werd bevestigd. 
In hoofdstuk 7 hebben we de samenvloeiing van viscoelastische druppels bestudeerd. Het splitsen van een druppel in twee kleinere druppels verandert drastisch wanneer polymeren aan de vloeistof worden toegevoegd. Door de vervorming van de polymeren tijdens het proces verschijnt een lange draad die de twee druppels met elkaar verbindt voordat ze uiteindelijk worden afgeknepen. In dit hoofdstuk hebben we laten zien hoe elasticiteit de samenvloeiing (de tegenhanger van splitsen) van twee druppels beïnvloedt. We onthulden dat dit gepaard gaat met een elastische singulariteit, die gekenmerkt wordt door een divergerende kromming van het druppeloppervlak op het punt van samenvloeiing. Intrigerend genoeg, ontdekten we dat de polymeren de temporele groeieigenschappen van de brug nauwelijks beïnvloeden, terwijl de ruimtelijke eigenschappen sterk beïnvloedt worden. Deze resultaten werden verklaard met behulp van een nieuwe viscoelastische zelfgelijkvormige analyse.

In hoofdstuk 8 hebben we de botsingen tussen twee druppels met verschillende oppervlaktespanningen bestudeerd. Met behulp van experimentele en numerieke technieken hebben we onthuld dat de asymmetrische druppelvorm die wordt waargenomen tijdens dergelijke botsingen wordt veroorzaakt door asymmetrische capillaire golven die het resultaat zijn van de verschillende oppervlaktespanningen van de druppels. We toonden aan dat de asymmetrie wordt versterkt bij toename van het oppervlaktespanningsverschil, en wordt onderdrukt door de traagheid van de botsende druppels te vergroten. Verder hebben we de capillaire golven bestudeerd op samenvloeiiende druppels in de limiet van geen traagheid. We hebben laten zien dat de asymmetrie niet direct wordt veroorzaakt door Marangoni-krachten. Integendeel, de asymmetrie wordt sterk verminderd door het Marangoni-effect. In plaats daarvan liggen de verschillende intrinsieke capillaire golfamplitudes en snelheden die samenhangen met de verschillende oppervlaktespanningen van de druppels aan de oorsprong van de asymmetrie die wordt waargenomen tijdens druppelbotsing en samenvloeiing.

Ten slotte hebben we in hoofdstuk 9 onze conclusies gepresenteerd en verschillende gebieden voorgesteld die interessant kunnen zijn voor toekomstig onderzoek. De resultaten die zijn gepresenteerd in dit proefschrift kunnen dienen als inspiratie voor de verdere verbetering van de inkjetprinttechnologie en de ontwikkeling ervan naar toepassingen die verder gaan dan het printen van documenten. 



\section{Acknowledgements}

Thorlabs table no. 35, my domain in the lab, is empty; my time in the Physics of Fluids group has almost come to an end. I look back on a wonderful time, first as a MSc student, then as a PhD candidate. I enjoyed collaborations with many researchers, and was supported by many colleagues, friends, and family members. In the final part of this thesis, I would like to give my heartfelt thanks to everyone who supported me during this period.

Jacco, I am deeply grateful for everything that you have done for me. I still remember our visit to Canon in 2017, which turned out to be an important step towards this thesis - we discovered our shared appreciation of Dire Straits on that day! Unfortunately, four years later, our much-desired band reunion seems more unlikely than ever... On a more serious note, it was on that day (in my memory at least) that we agreed to start the shared journey that led to this thesis - a thesis that I would never have been able to write without your skilful guidance. You are an excellent teacher, able to explain complicated matter in simple terms with unmatched enthusiasm. Beyond just presenting the solution to a problem, you explain the reasons 'why' a solution is, indeed, the correct solution. In the lab you gave me a lot of freedom. I was able to play around with ideas, perform preliminary experiments, and define my own research questions. You also always maintained a realistic view of experiments - of what is possible, and what is not. I think the biggest compliment that I can make is this: Every single time that I left your office (or ended a Teams call) after a meeting, I felt more motivated, inspired, and more enthusiastic about our research - I think that is the signature of a great supervisor.

Tim, I am also deeply grateful for your guidance. You taught me many experimental skills that I did not posses at the start of this four-year journey. For that reason alone, this thesis would not have existed without you. You contributions reach far beyond that though, not least because you co-coordinated the FIP project. I always enjoyed our (semi-)regular meetings, which provided a great way to structure my thoughts. They also provided a great opportun- 
ity to chat about matters unrelated to our direct research. Furthermore, you always had great suggestions for our research, and came up with solutions to problems that I encountered in the experiments (for example, suggesting the use of Microdrop pipettes in chapter 2!). You always strive for the best quality when it comes to research, and inspired me to do so too. At the same time, you also gave me a lot of freedom in defining research directions. I am very glad, and proud, that you are my copromotor.

Herman, your influence on my thesis was huge. You provided us with many interesting phenomena that you encountered in inkjet printing, which inspired many chapters in this thesis. Beyond that, you also provided insightful comments and asked important questions, both of which led to the improvement of our collaborative work. Thank you!

Marjolein, we first met when I was starting to explore the combined effects of colloidal destabilisation and hydrodynamics. Your encouragement to keep researching this system (despite the challenges that it posed) and your suggestions were crucial to get this project to where it is now. Apart from that, your feedback was crucial to many aspects of this thesis. I very much enjoyed our collaboration. Thank you so much!

Detlef, you have built an astounding research group. The facilities and collective knowledge in the Physics of Fluids Group (and the FIP project) provide an unmatched environment for research. Thank you.

There are several other physicists who have shaped my . In chronological order: Kari, my first experience with "real" state-of-the-art in-the-lab-physics was under your supervision during my visit in Canada as a master student. Together with Raff you taught me the skills that a soft matter physicist requires. Your enthusiasm is for physics is infectious. I am deeply grateful for everything that you (and Raffi) taught me in Canada. Maxime, you took me under your wing when I returned to the Netherlands. You showed me the inner workings of the PoF laboratories and taught me a tremendous amount of experimental skills. I am very happy that we got to work together and I give you my heartfelt thanks for everything that you have done for me. Stefan, you are truly a master of all trades. I greatly enjoyed our meeting in Göttingen, and all the fruitful discussions that we had. Your impact on my thesis was huge, thank you so much!

The FIP project was an excellent opportunity to meet new people, and it led to several successful collaborations. Yaxing, my friend, we shared so much during our PhDs. I admire your drive and passion for science. I wish you and your family all the best! Maaike, you were always up for an enjoyable chat in 
the lab, thanks! Wojtek and Stefan Kooij, our collaboration with Olinka on vicinal alkanediols turned out very nice, thanks! Qingguang and Jens, thank you for your efforts on our shared project on liquid lens coalescence. Christian, your skills with numerical simulations are incredible, and your physical knowledge and intuition are very impressive. I enjoyed our collaboration on droplet collisions, but also all the discussions we had about other topics. Thank you so much - also for your coordination of the FIP project! To all others involved in the FIP project: thank you for the interesting discussions!

The support staff of PoF, Joanita, Bas, Gert-Wim, Martin B, Thomas, and Dennis $v G$ : without you the group would certainly not operate as smoothly as it does now. Thank you for organising, buying, building, and fixing things, and for keeping us safe inside the labs!

I had great officemates in ME214B, a small office that is hidden away in a quiet corridor. Álvaro, I enjoy your light-heartedness, which brought many laughs to the office. Your music taste though... Liz, thank you for the many lunch walks and our discussions on all aspects of life, it was a pleasure to share an office with you! Pablo, though our office hours were not always perfectly synchronised, we did share great moments, thanks! Uddalok, as the newest office member, you took it upon yourself to clean up the huge pile of junk that accumulated over many years in our office cabinet. I can only express my deepest gratitude for your service to the ME214B community. Joking aside, you were a great office- and labmate, thanks! And hey, I still owe you that beer...

I was lucky enough to go on several holiday trips before and after conferences that I attended during my PhD. CS and Sin Dee, Maaike, Pallav and Adyasha, Wojtek, Myrthe, and Yaxing: our trip to Manchester was great, highlights include getting muddy on Kinder Scout and visiting the birthplace of The Communist Manifesto in Chetham's Library. Jelle, Utkarsh, Srinidhi, Peter, Dennis vG, Vamsi, Martin KS, Martin A, Pieter, and Pim B: I greatly enjoyed our trip to the Nantahala lake house. On my final trip, I visited Seattle and its beautiful surroundings together with Myrthe, Pieter, and Pim B. Myrthe, you are always full of joy and even fuller of energy. Thank you for the many walks and talks! Pieter, will Dumoulin ever win the Tour? (Probably not.) Will Kelderman manage to stay upright on his bike for three weeks straight? (Definitely not.) We should discuss these important questions at some point, perhaps whilst enjoying some delicious peanut butter sandwiches. Pim B, your poultry cooking skills are unmatched, and I admire your relaxed attitude towards life; please teach me how to be so zen. 
To the Dynamics team, Jacco, Alvaro, Pieter, Martin E, Charlotte, and Pim D: I enjoyed working with you, and I learned a lot from all of you when it comes to dynamics and (especially) educating students, thank you!

I (co-)supervised several students during my PhD. Mees, I greatly enjoyed working (and learning!) together with you and your co-supervisor Walter on the theory of contact angles in the presence of surfactants. Patrick, we challenged you with a tricky droplet collision study, and you managed to perform excellent experiments, thanks! Bas, your project, that I co-supervised with Carola, was full of unforeseen difficulties, but you did a great job tackling them, I wish you all the best. Pim D, your experiments on viscoelastic coalescence are beautiful, thank you!

There are several people in PoF who I still want to thank. Diana, it took half of our PhDs before we really got to know each other, but I think that we made up for lost time. Chatting in the lab and listening to your 'great' jokes is always a delight. Thank you for all the great times! Charu, jouw vermogen om snel de betekenis en structuur van wiskundige vergelijkingen te begrijpen (en talen te leren!) is verbazingwekkend-ik zou deze magische vaardigheid graag zelf hebben! Ik heb ook genoten van onze gesprekken, met name tijdens onze reis naar Münster. Walter, I admire you desire to fully grasp problems. Thank you for teaching me so much about theory. Ambre, you are an extremely skilled experimentalist. Thank you for all the help with my students and our collaboration on viscoelastic coalescence. Jessica, thank you for all the compliments on my shirts and sweaters and all the homemade Canadian baked goods! Carola, thanks for inviting me to join your great idea for a master project. I always enjoyed our collaborations and chats. And thanks for setting up so many nice initiatives (like the Soft Matter Seminar)! Kirsten, thank you for your work on our coalescence project. Sander, thank you for providing the $\mathrm{IAT}_{\mathrm{E}} \mathrm{X}$ thesis template, I think it saved me a lot of frustration! Finally, I thank all other PoF members for the many (non-)scientific discussions and fun times!

I had the pleasure to collaborate with many people on various projects; all but one have been named. Gunnar, your sharp analysis encouraged us to have another look at our viscous lens coalescence work, and your ideas and simulations improved the work a lot. Thank you!

Mijn Zwolse vrienden - al meer dan vijftien jaar trekken wij met elkaar op. Jorinde, Stijn, Dennis, Tess, ik ben jullie ontzettend dankbaar dat we na zoveel jaren nog steeds zo goed bevriend zijn, op naar de volgende vijftien!

Dan mijn vrienden uit Enschede - wie had gedacht dat een zondagavond 
televisiekijken tot zo'n hechte vriendengroep zou leiden? (ir.) Joël, Sanne, Frank, Seray, Dennis, Ines, het is altijd bijzonder fijn om met jullie samen te zijn.

Jorinde en Joël, dank dat jullie mij op het moment suprême als paranimfen bijstaan.

Han, Janny, en David, dank voor jullie interesse in mijn werk, en voor de fantastische vakanties in De Haan en Eindhoven.

Tot slot, Sjoerd, paps en mams, dank voor jullie onvoorwaardelijke steun, vertrouwen, interesse, en alles dat jullie voor mij gedaan hebben. Het doet me goed dat ik bij jullie altijd terecht kan voor een goed gesprek, een goed advies, en (het allerbelangrijkste) een veilige thuishaven. 


